\title{
Optimización Evolutiva y Multiobjetivo en base a criterios RAMS+C para Centrales Nucleares
}

TESIS DOCTORAL

Presentada por:

Jose F. Villanueva López

Dirigida por: Dr. I. I. Sebastián Martorell Alsina

Dr. I. I. Sofía Carlos Alberola

Valencia, Julio de 2011 

A mi padre 

Agradecimiento a todos aquellos que me han ayudado a hacer posible esta tesis, muy especialmente a Sofia, Sebastián, Ana y Vicente por su apoyo, dedicación y confianza en mí.

A Doña Encarnación, porque uno recibe siempre aquello que da.

A todos aquellos que día a día están conmigo y aguantan mis momentos en las tertulias de las comidas.

A mis amigos, tan fundamentales en mi equilibrio personal, aunque no siempre les pueda ver tanto como quisiera.

A mis compañeros de viaje, de los que tanto aprendo sobre las leyes que rigen la existencia y a ser dueños de nuestras vidas.

A mi familia por ser mi apoyo, mis hermanos, cuñados, sobrinos y ahijados, y en especial a mi hermana por ser la primera en confiar en mí, y a mi madre que siempre ha estado y estará a mi lado y a la que tanto debo en mi forma de ser.

A todos mis más sincero agradecimiento.

Esto es vuestro también. 



\section{RESUMEN}

En el ámbito de las centrales nucleares existe un gran interés en el estudio de las características de fiabilidad, mantenibilidad y disponibilidad de sus equipos y su influencia en la seguridad y la economía de las centrales.

Por todo ello, la toma de decisiones sobre la mejora y, en su caso, optimización de las pruebas y del mantenimiento en centrales nucleares se puede mejorar con la consideración simultánea de criterios RAMS $+C$. EI proceso para encontrar los procedimientos de pruebas y mantenimientos óptimos debe equilibrar los logros alcanzados en base a los criterios RAMS $+\mathrm{C}$.

El reto que plantea la presente tesis es, por un lado, la necesidad de formular nuevos modelos que permitan representar de forma explícita el efecto de las pruebas y mantenimientos sobre dichos criterios RAMS $+C$. En segundo lugar, se requiere del estudio de la viabilidad de nuevos métodos de optimización capaces de manejar de forma adecuada tanto la complejidad de los nuevos modelos desarrollados como el gran número de variables de decisión que pueden estar involucradas en el proceso de optimización en base a múltiples criterios RAMS $+C$ y además, que sean capaces de obtener buenos resultados en la optimización en presencia de incertidumbres asociadas tanto a parámetros, modelos o variables de decisión, y que afectan a la toma de decisiones. Para la resolución del problema de optimización multicriterio que se plantea se ha seleccionado como herramienta de optimización evolutiva los algoritmos genéticos, los cuales han mostrado una alta eficiencia en la resolución de problemas complejos.

El objetivo pues de la presente tesis se centra en el desarrollo de nuevos modelos y métodos necesarios para acometer el proceso de optimización de pruebas y mantenimientos en base a criterios RAMS $+C$, con aplicación a sistemas de seguridad de centrales nucleares.

Se han propuesto varias aproximaciones que modelan el comportamiento de los equipos con independencia temporal, es decir, mediante el uso de valores medios para los atributos RAMS $+C$. En la siguiente etapa, se progresa en la dirección de proponer nuevos modelos que tienen en cuenta la variable tiempo en el comportamiento de los equipos desde el punto de vista RAMS $+C$. Ello requiere, por un lado adaptar los anteriores algoritmos de optimización para manejar nuevos criterios y variables de decisión, y además integrar mejoras en los algoritmos de optimización que los haga más eficientes. 
Por último, en la tercera etapa, se proponen nuevos modelos y métodos que consideran el efecto de las incertidumbres asociadas a los parámetros del modelo, al propio modelo, e incluso a las variables de decisión en el proceso de optimización.

En las tres etapas se aportan casos de aplicación de los nuevos modelos y métodos que demuestran la viabilidad y aplicabilidad de los mismos. 


\section{ABSTRACT}

In the field of nuclear power plants there is a great interest in the study of the characteristics of reliability, maintainability and availability of their equipment and its influence on the security and the economy of the plant.

Therefore, the decision making on improvement and, even optimization, of testing and maintenance activities at nuclear power plants can take be better solved from the simultaneous consideration of criteria RAMS $+C$. Therefore, the process to find optimal tests and maintenance strategies should balance the goals achieved, based on RAMS $+C$ criteria.

The challenge of this thesis is, firstly, the need to develop new models to explicitly represent the impact of maintenance and test on RAMS $+C$ criteria. Secondly, development of new optimization methods capable of properly handling both, the complexity of the new models developed, as a large number of decision variables may be involved in the optimization process based on multiple RAMS $+C$ criteria, and also the presence of uncertainties that affect the decision-making process, associated to parameters, models or decision variable. Genetic Algorithms have been chosen to find a solution of the multicriteria optimization problem, as they have already shown a high efficiency in solving complex problems.

The objective of this thesis focuses on the development of new models and methods necessary to undertake testing and maintenance optimization process based on RAMS $+C$ criteria, and their application to nuclear plants safety related systems.

There exists several approaches that model the behavior of the systems with time independence, i.e. through the use of average values for RAMS $+C$ attributes have been proposed. Next stage, proposes new RAMS $+C$ models to consider time in the behavior of the systems. This requires, the customizations of genetic algorithms previously used in the optimizations to handle new criteria and decision variables, and also the integration of improvements in the algorithms Finally, new models and methods that consider the effect of uncertainties associated with the parameters, models and, and even the decision variables in the optimization process are proposed. All the new proposals are analyzed in different cases of application that demonstrate the feasibility and applicability of the new models and methods. 



\section{RESUM}

En l'àmbit de les centrals nuclears hi ha un gran interès en l'estudi de les característiques de fiabilitat, mantenibilitat i disponibilitat dels seus equips i la seua influència en la seguretat i l'economia de les centrals.

Per tot això, la presa de decisions sobre la millora i, si és el cas, optimització de les proves i del manteniment en centrals nuclears es pot millorar amb la consideració simultània de criteris RAMS $+C$. Per tant, el procés per a trobar els procediments de proves i manteniments òptims ha d'equilibrar els èxits aconseguits basant-se en els criteris RAMS $+\mathrm{C}$.

El repte que planteja la present tesi és, d'una banda, la necessitat de formular nous models que permeten representar de forma explícita l'efecte de les proves i manteniments sobre els criteris RAMS $+\mathrm{C}$. En segon lloc, es requerix l'estudi de la viabilitat de nous mètodes d'optimització capaços de tractar de forma adequada tant la complexitat dels nous models desenvolupats com el gran nombre de variables de decisió que poden estar involucrades en el procés d'optimització basant-se en múltiples criteris RAMS $+C \mathrm{i}$, a més, que siguen capaços d'obtindre bons resultats en l'optimització en presència d'incerteses associades tant a paràmetres com a models 0 a variables de decisió, que afecten la presa de decisions. Per a la resolució del problema d'optimització multicriteri que es planteja s'ha seleccionat com a ferramenta d'optimització evolutiva els algoritmes genètics, els quals han mostrat una alta eficiència en la resolució de problemes complexos.

L'objectiu per tant de la present tesi se centra en el desenvolupament de nous models i mètodes necessaris per a l'optimització de proves i manteniments basant-se en criteris RAMS $+C$, amb aplicació a sistemes de seguretat de centrals nuclears.

S'han proposat diverses aproximacions que modelen el comportament dels equips amb independència temporal, és a dir, per mitjà de l'ús de valors mitjos per als atributs RAMS $+C$. En la següent etapa, es progressa en la direcció de proposar nous models que tenen en compte la variable temps en el comportament dels equips des del punt de vista RAMS $+C$. Això requerix, per un costat adaptar els anteriors algoritmes genètics per a tractar nous criteris i variables de decisió, i a més integrar millores en els algoritmes de optimització que els faça més eficients.

Finalment, en la tercera etapa, es proposen nous models i mètodes que consideren l'efecte de les incerteses associades als paràmetres del model, al propi model, i inclús a les variables de decisió en el procés d'optimització. 
En les tres etapes s'aporten casos d'aplicació dels nous models i mètodes que demostren la viabilitat i aplicabilitat dels mateixos. 


\section{Contenido}

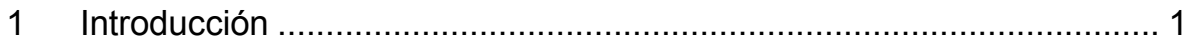

1.1 Antecedentes y motivación ....................................................... 1

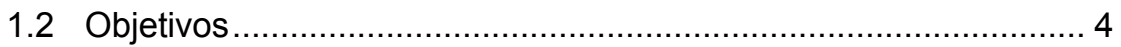

1.3 Resumen de las aportaciones y organización de la tesis ............. 4

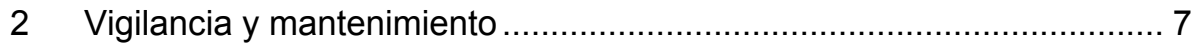

2.1 Tipos de vigilancia y mantenimiento ………........................... 7

2.2 Tipos de causas de fallo y su relación con la vigilancia y el mantenimiento ........................................................................ 11

2.3 Requisitos de los modelos de cuantificación RAMS $+C$ adaptados a pruebas y mantenimiento .................................... 14

2.4 Papel de Pruebas, Mantenimientos e Incidencias en criterios RAMS+C

2.5 Programas para la mejora de Pruebas y Mantenimiento............. 22

3 Modelado de parámetros RAMS+C ................................................ 25

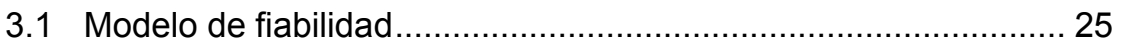

3.1.1 Modelo PAS. ........................................................... 27

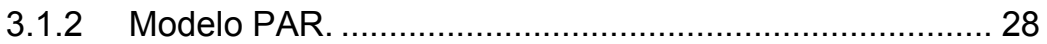

3.2 Efecto de las actividades de vigilancia y mantenimiento sobre

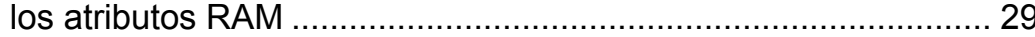

3.3 Modelos de mantenibilidad ....................................................... 30 


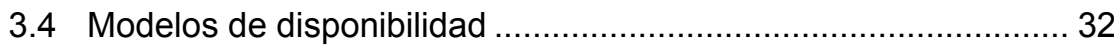

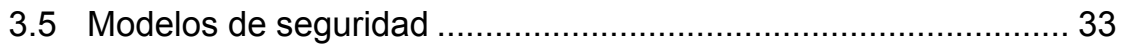

3.6 Modelos de coste.................................................... 34

3.7 Incorporación de la dependencia temporal .............................. 36

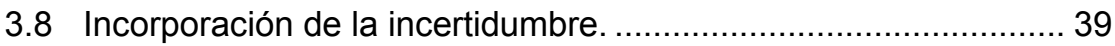

3.8.1 Incertidumbre en el modelo de fiabilidad y mantenibilidad .................................................... 40

3.8.2 Incertidumbre de parámetros ................................ 42

4 Algoritmos evolutivos en optimización .................................... 45

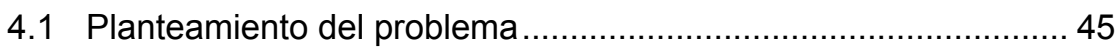

4.1.1 Problema de Optimización Simple Objetivo. Solución única ................................................................. 49

4.1.2 Problema de Optimización Multiobjetivo. Múltiples soluciones. Conjunto de soluciones óptimas no dominadas de Pareto y frontera de Pareto. .................. 49

4.2 Toma de decisiones en base a criterios RAMS+C .................. 50

4.3 Optimización mediante computación evolutiva: Algoritmos

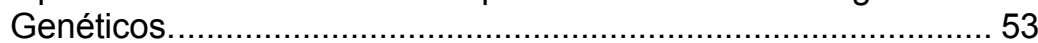

4.3.1 Orígenes, fundamentos y primeras aplicaciones........... 53

4.3.2 Evolución de los Algoritmos Genéticos........................ 54

4.3.3 Desarrollo de Algoritmos Genéticos Multiobjetivo........... 55

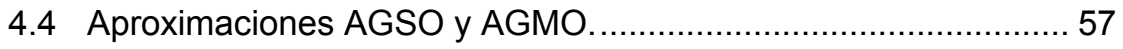

4.4.1 AGSO basado en la efectividad .............................. 58 
4.4.2 AGMO basado en SPEA2......

61

4.5 Consideración de incertidumbres en la toma de decisiones.

4.5.1 Principios de la toma de decisiones con incertidumbres.

4.5.2 Formulación del problema de optimización considerando incertidumbres

5 Optimización multiobjetivo de requisitos STI y AOT a nivel de sistema y planta

5.1 Descripción del problema ....................................................... 79

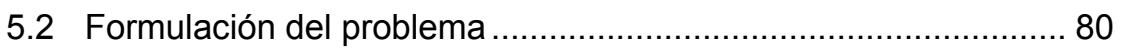

5.3 Método de resolución del problema y resultados......................... 83

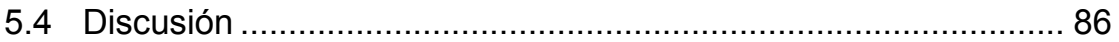

6 Optimización multiobjetivo de requisitos de vigilancia a nivel de sistema considerando dependencia temporal

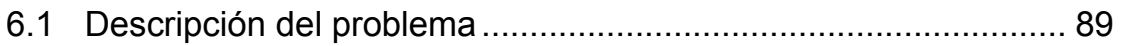

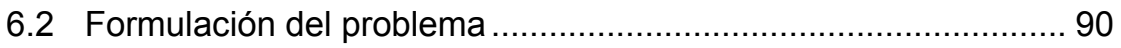

6.3 Método de resolución y resultados ............................................. 96

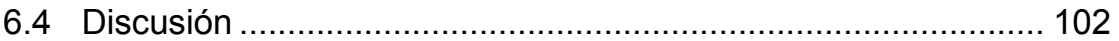

7 Optimización multiobjetivo de la vigilancia y mantenimiento a nivel de sistema considerando el efecto de las incertidumbres.

7.1 Optimización considerando incertidumbres en las variables de

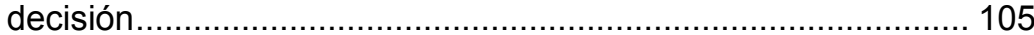

7.1.1 Descripción del problema............................................. 105 
7.1.2 Formulación del problema........................................... 106

7.1.3 Método de resolución y resultados .............................. 108

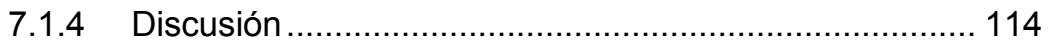

7.2 Optimización considerando incertidumbre de parámetros......... 116

7.2.1 Descripción del problema............................................ 116

7.2.2 Formulación del Problema ........................................... 116

7.2.3 Método de resolución y resultados .............................. 120

7.2.4 Discusión ................................................................ 130

7.3 Optimización considerando incertidumbres de modelo de mantenimiento imperfecto. .................................................... 133

7.3.1 Descripción del problema............................................. 133

7.3.2 Formulación del problema.............................................. 133

7.3.3 Método de resolución y resultados ............................. 137

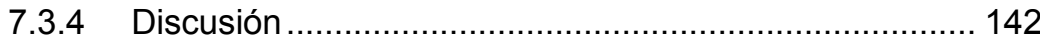

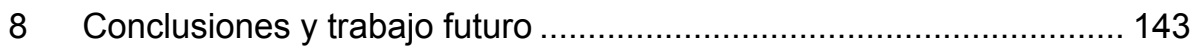

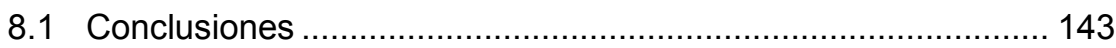

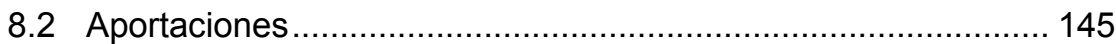

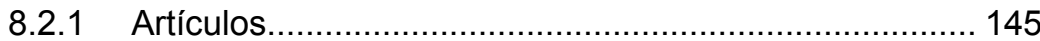

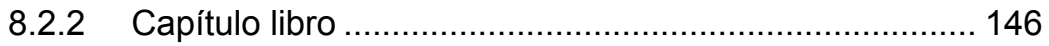

8.2.3 Congresos Internacionales......................................... 147

8.2.4 Congresos Nacionales ……………………................. 149 


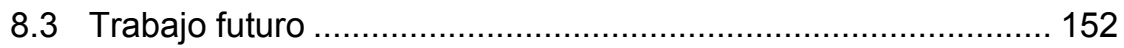

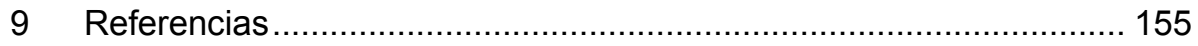





\section{Figuras}

Figura 2-1: Tipos de actividades de pruebas y mantenimientos en las centrales nucleares.

Figura 2-2: Condición funcional del equipo frente a actividades de vigilancia y mantenimiento

Figura 2-3: Relación entre causas de fallo y las actividades de mantenimiento.

Figura 2-4: Estrategias de mantenimiento por agregación de tareas.

Figura 2-5: Incidencia del diseño, la vigilancia y el mantenimiento en atributos RAMS+C.

Figura 2-6: Pruebas y mantenimiento en relación a las ETFs de las centrales nucleares.

Figura 2-7: Programas implementados para la mejora de ETFs y mantenimiento en Centrales Nucleares.

Figura 3-1: Diagrama de estados funcionales, actividades de mantenimiento, pruebas y transiciones entre equipos de seguridad.

Figura 3-2: Esquema de la mantenibilidad de un equipo para una tarea A. 32

Figura 4-1: Estrategias de resolución de Problemas de Optimización Multi-Objetivo

Figura 4-2: Espacio objetivo de las posibles soluciones para el AGSO basado en la efectividad.

Figura 4-3: Principales componentes del AGMO basado en el SPEA2 62

Figura 4-4: Dependencia del nivel de confianza $\beta$ respecto al número de muestras con varios niveles de tolerancia y para el intervalo de tolerancia unilateral. 
Figura 5-1: Soluciones no dominadas: coste - riesgo simple contribución al riesgo anual.

Figura 5-2: Soluciones no dominadas: coste - no fiabilidad indisponibilidad detectada.

Figura 6-1: Sistema de inyección a alta presión. ....................................... 92

Figura 6-2: Indisponibilidad dependiente del tiempo. Caso Inicial ............... 96

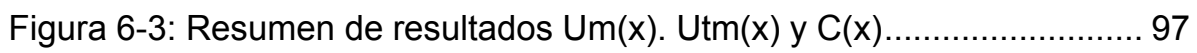

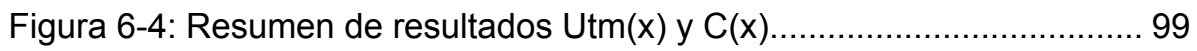

Figura 7-1: Resultados obtenidos considerando distribución uniforme ..... 110

Figura 7-2: Resultados obtenidos considerando una distribución normal. 111

Figura 7-3: Resultados obtenidos considerando una distribución lognormal

Figura 7-4: Gráfico Box and Whisker para T1 dependiendo del modelo de distribución

Figura 7-5: Gráfico Box and Whisker para T1 dependiente de la máxima tolerancia admitida.

Figura 7-6: Gráfica U-C de la incertidumbre de los resultados considerando dependencia entre $\mathrm{U}$ y $\mathrm{C}$.

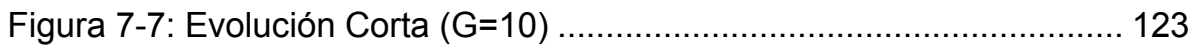

Figura 7-8: Evolución Mediana (G=50) …......................................... 124

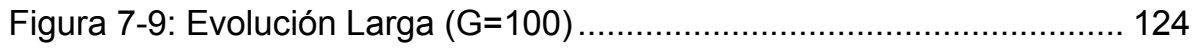

Figura 7-10: Gráfica U-C de resultados medios del análisis de sensibilidad de $\mathrm{G}$ frente a N para dos parejas comparables. .. 126

Figura 7-11: Gráfico U-C de resultados con incertidumbre considerando dependencia entre $\mathrm{U}$ y $\mathrm{C}$ y funciones objetivos basadas en 
valores medios.

Figura 7-12: Gráfico U-C de resultados con incertidumbre del análisis de sensibilidad para $\mathrm{G}$ con $\mathrm{N}$ constante y considerando dependencia entre $\mathrm{U}$ y $\mathrm{C}$ y funciones objetivo basados en valores medios

Figura 7-13: Gráfico U-C de resultados medios para los problemas de optimización simple y múltiple

Figura 7-14: Análisis de sensibilidad de los valores medios en N para POS de minimización del límite superior de tolerancia para indisponibilidad de sistemas, esto es AGSO-U (solo se muestra la zona de interés)

Figura 7-15: Análisis de sensibilidad de los valores medios en $\mathrm{N}$ para POS de minimización del límite superior de tolerancia para el coste del sistema, esto es AGSO-C (solo se muestra la zona de interés)

Figura 7-16: Resultados de la optimización considerando incertidumbre en el parámetro $\varepsilon$.

Figura 7-17: Análisis de sensibilidad para la selección del modelo de mantenimiento imperfecto.

Figura 7-18: Comparación de los resultados de optimización considerando $\varepsilon$ y $\varepsilon+$ incertidumbre en el modelo de mantenimiento imperfecto. 



\section{Tablas}

Tabla 2-1: Resumen de requisitos de modelización de disparo y efectos sobre RAM relacionados con las actividades T\&M.

Tabla 4-1: Tamaño muestral $\mathrm{N}$ para una variable e intervalo unilateral. 71

Tabla 4-2: Tamaño muestral $\mathrm{N}$ para dos funciones objetivo e intervalo unilateral.

Tabla 5-1: Datos de fallos para generadores diesel de emergencia.

Tabla 5-2: Datos de costes para generadores diesel de emergencia.

Tabla 5-3: Restricciones en las variables de decisión

Tabla 5-4: Resultados de la optimización

Tabla 6-1: Datos típicos para bombas y válvulas, e intervalos actuales de pruebas, mantenimientos y reemplazamientos

Tabla 6-2: Datos típicos de costes para válvulas y bombas

Tabla 6-3: Grupos de estrategias agrupados en función del tiempo de la primera prueba (TP) e intervalos entre pruebas (T) del sistema de inyección a alta presión.

Tabla 6-4: Descripción de los casos de ejecución .98

Tabla 6-5: Soluciones relevantes para el intervalo entre pruebas y estrategia de las pruebas bajo varios casos

Tabla 7-1: Intervalo entre pruebas para el Sistema de inyección a alta presión.

Tabla 7-2: Condiciones iniciales y rango de valores permitidos para las variables de decisión. 
Tabla 7-3: Parámetros adoptados para el AGMO ajustado al método de intervalos de tolerancia.

Tabla 7-4: Grupos de estrategias de intervalos entre pruebas y mantenimientos preventivos para el sistema de inyección de agua a presión

Tabla 7-5: Condiciones iniciales y rango de valores permitidos para las variables de decisión.

Tabla 7-6: Resultados para los valores máximos de $\rho_{m}$ y $\lambda_{0 m}$. 120

Tabla 7-7: Parámetros adoptados para los AGMO y SOGA particulares .....121

Tabla 7-8: Parámetros adoptados para el AGMO ajustado al método de intervalos de tolerancia. 


\section{Acrónimos}

\begin{tabular}{ll} 
AA.GG & Algoritmos Genéticos \\
AGMO & Algoritmo Genético Multiobjetivo \\
AGSO & Algoritmo Genético Simple objetivo \\
APS & Análisis Probabilístico de Seguridad \\
AOT & Allowed Outage Time \\
BAO & Bad as Old \\
CB & Conditioned-based / Por condición \\
CLO & Condiciones Límite de Operación \\
CM & Corrective Maintenance / Mantenimiento Correctivo \\
CC.NN & Centrales Nucleares \\
CO & Continuous / Monitorización Continua \\
DBA & Design Based Accidents \\
ETF & Especificaciones Técnicas de Funcionamiento \\
GAN & Good as New \\
HPIS & High Pressure Injection System \\
LMP & Life Management Program \\
LRA & License Renewing Amendment \\
MRI & Maintenance Rule Implementation \\
\hline
\end{tabular}




$\begin{array}{ll}\text { NE } & \text { Normalmente en Espera } \\ \text { NSGA } & \text { Non-dominated Sorting Genetic Algorithm } \\ \text { OF } & \text { On Failure / Por Fallo } \\ \text { PAM } & \text { Pro-Active Maintenance / Mantenimiento Pro-Activo } \\ \text { PAR } & \text { Proportional Age Reduction } \\ \text { PAS } & \text { Proportional Age Setback } \\ \text { PDM } & \text { Predictive Maintenance / Mantenimiento Predictivo } \\ \text { POMO } & \text { Problema de Optimización Multiobjetivo } \\ \text { POSO } & \text { Problema de Optimización Simple Objetivo } \\ \text { PVM } & \text { Preventive Maintenance / Matenimiento Preventivo } \\ \text { RAMS+C } & \text { Reliability, Availability, Maintainability and Safety plus Cost } \\ \text { RCM } & \text { Reliability Centered Maintenance } \\ \text { RI-ISI } & \text { Risk Informed In Service Inspection } \\ \text { RI-IST } & \text { Risk Informed In Service Testing } \\ \text { RI-LB } & \text { Risk Informed Licensing Basis } \\ \text { RI-LCO } & \text { Risk Informed Limit Conditions of Operation } \\ \text { RI-SR } & \text { Risk Informed Surveillance Requirement } \\ \text { RI-TS } & \text { Risk Informed Technical Specification } \\ \text { RM } & \text { Replacement Maintenance / Mantenimiento Sustitución } \\ \text { RV } & \text { Requisitos de Vigilancia } \\ & \end{array}$




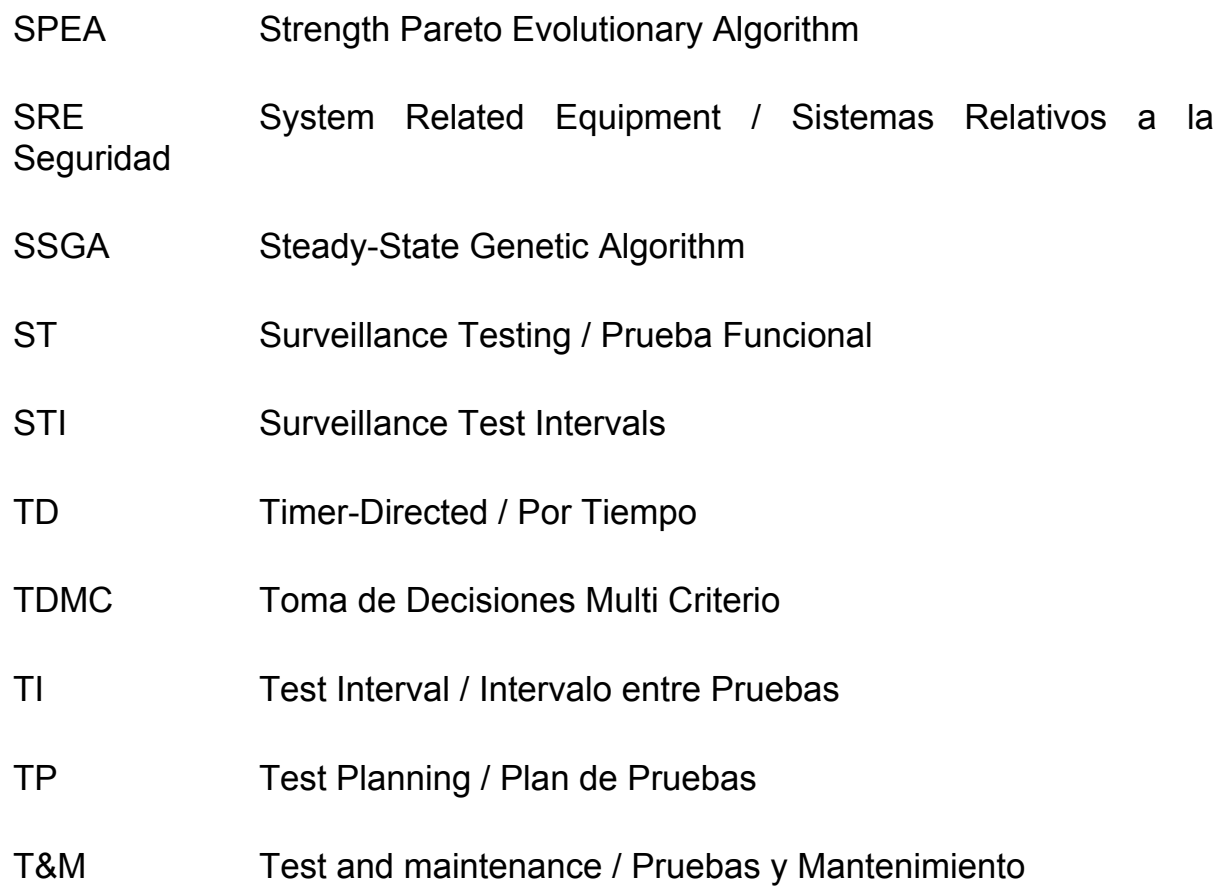



Haz lo necesario para lograr tu más ardiente deseo, y acabarás lográndolo Beethoven, Ludwig van 



\section{Introducción}

\subsection{Antecedentes y motivación}

Durante los últimos años la industria nuclear ha mostrado un creciente interés en todo lo relativo al estudio de las características de fiabilidad, mantenibilidad y disponibilidad de sus equipos y su influencia en la seguridad y la economía de la central.

En este contexto destacan dos grandes líneas de actuación. La primera de ellas tiene como objetivo mejorar dichas características abreviadas por las siglas anglosajonas RAMS $+C$ (Reliability, Availability, Maintainability and Safety plus Cost), desde el punto de vista del diseño de equipos que de manera inherente mejoren los niveles de cada una de estas características. La segunda línea se centra en analizar y optimizar el papel de las pruebas y el mantenimiento de equipos como vía de actuación para garantizar que dichas características inherentes se mantienen de manera aceptable a lo largo de la vida de la central.

El estudio de la situación actual de las centrales nucleares (CC.NN.) muestra la coexistencia de diferentes programas implantados en la planta, la mayoría enmarcados dentro de la segunda línea de actuación. En particular, estos programas buscan la mejora de parámetros particulares de las Especificaciones Técnicas de Funcionamiento (ETFs), donde se enmarcan las pruebas de vigilancia de sistemas de seguridad, y del mantenimiento. 
De acuerdo con los objetivos establecidos en estos programas, es posible dividirlos en dos grupos diferentes, unos centrados en la mejora de las bases de licencia y otros enfocados a la mejora de las actividades de mantenimiento. En el primer grupo se incluyen los programas de mejora de las Especificaciones Técnicas de Funcionamiento informadas en el riesgo (Lakkso 1990), (Atefi 1991), (IAEA TECDOC 729), (RG 1177) y (RG 1174) los cuales se centran en la mejora de parámetros relativos a las especificaciones técnicas (Risk Informed Technical Specification, RI-TS), en particular en los requisitos de tiempos máximos permitidos de inoperabilidad, (Allowed Outage Time, AOT) e intervalos entre pruebas (Surveillance Test Intervals, STI) incluidos en las Condiciones Límite de Operación, CLO (denominadas Risk Informed Limit Conditions of Operation, RI-LCO) y los Requisitos de Vigilancia, RV (denominados Risk Informed Surveillance Requirement, RISR) respectivamente (Samanta 1988), (Vesely 1999), (Kim 1992), (Mankamo 1993), (Vaurio 1995), (Kim 1994), (Martorell 1995), (Samanta 1995). Estos programas junto con otros similares, tales como los centrados en la vigilancia en servicio (denominados Risk Informed In Service Inspection, RI-ISI) o los centrados en las pruebas en servicio (denominados Risk Informed In Service Testing, RI-IST), trabajan principalmente con criterios de seguridad, tales como el incremento de riesgo condicionado, el riesgo simple y la contribución al riesgo anual de la planta, donde los objetivos numéricos se han establecido para limitar el efecto que los cambios en las ETFs pueden tener sobre el riesgo para la salud y seguridad de la población. Estos límites se establecen en base a las guías reguladoras (RG 1174) y (RG 1177).

El segundo grupo de programas se centra en la mejora de la política de mantenimiento de la central. Un objetivo común de estos programas es alcanzar y mantener una alta fiabilidad intrínseca, reduciendo, tanto como sea posible, la tasa de fallo, que adquiere mayor relevancia para las estructuras, sistemas y componentes importantes para la seguridad de la central. Éste es uno de los principales objetivos en la implantación de un programa de Mantenimiento Centrado en la Fiabilidad (Reliability Centered Maintenance, RCM) (Taylor 1989), (EPRI NP 6152), (Martorell 1996). Sin embargo, el hecho de dejar los equipos fuera de servicio por mantenimiento provoca que no estén disponibles para desempeñar su función de mitigación o prevención de un accidente en caso de que se requiera su funcionamiento. Ambos atributos, fiabilidad y mantenibilidad, son cruciales para la seguridad de la planta, y el mantenimiento tiene efectos opuestos en ambos atributos, por lo que se busca equilibrar fiabilidad de equipos y mantenibilidad (o disponibilidad), siendo éste último el objetivo central perseguido al implantar el programa de la Regla de Mantenimiento (Maintenance Rule Implementation, MRI), (RG 1160), (RG 1182) y (Martorell 1999).

Además, el envejecimiento y la degradación es una consecuencia natural 
para la mayoría de los equipos de las centrales y, por lo tanto, su fiabilidad se degrada durante la vida operacional de la planta. Consecuentemente, la degradación de las estructuras, sistemas y componentes, en particular de aquéllos importantes para la seguridad de la planta, debe ser mantenida bajo control, siendo éste el principal objetivo del Programa de Gestión de Vida (Life Management Program, LMP) (IAEA-TECDOC-338), (Unesa 1995) e (Iberdrola GV-IN-001). La implantación del programa de gestión de vida ayuda además a mantener abierta la posibilidad de una renovación de la licencia de operación de las centrales nucleares, que es el objetivo de la implantación de un programa de Renovación de Licencia (License Renewing Amendment, LRA) (RG 1188) y (NEI 95-10).

En los programas RCM, MRI, y LMP se establecen objetivos numéricos asociados a los criterios de aceptación, que intentan controlar el impacto que las actividades de mantenimiento tienen en los atributos de fiabilidad, mantenibilidad y disponibilidad. Las guías reguladoras (RG 1160), (RG 1182) y (RG 1188) muestran ejemplos de dichos objetivos numéricos en relación con los programas involucrados en la política de mantenimiento y los atributos RAM (Reliability, Availability and Maintainability) para los equipos.

La coexistencia en la central de programas de mejora de las Especificaciones Técnicas de Funcionamiento y de la Política de Mantenimiento hace que existan efectos sinérgicos de los cambios que pueden afectar a varios programas y criterios de aceptación. Así, por ejemplo, un cambio en una CLO, afectará en el modo en que el mantenimiento puede ser llevado a cabo, por ejemplo, limitando el AOT, y podría requerir el establecimiento de medidas compensatorias en la gestión de las configuraciones de la planta. Por el contrario, la mejora en la política de mantenimiento basado en criterios RAM puede permitirnos un rango más amplio para posibles cambios en los requisitos de $\mathrm{CLO}$ o RV basados en criterios de seguridad.

Por todo ello, la toma de decisiones sobre la mejora y, en su caso, optimización de las pruebas y del mantenimiento en centrales nucleares se puede beneficiar de la consideración simultánea de criterios RAMS $+C$. Por ejemplo, la optimización de las actividades de pruebas y mantenimiento de sistemas relacionados con la seguridad apunta a la mejora de los atributos RAM, los cuales derivan en una mejora del nivel de seguridad de la planta. Esto, sin embargo, se obtiene a expensas de un incremento de inversión en los recursos (p.e. costes, recursos humanos, etc.). Por lo tanto, el proceso para encontrar los procedimientos de pruebas y mantenimientos óptimos debería equilibrar los logros alcanzados en base a los criterios RAMS $+C$.

El reto que plantea este nuevo enfoque es, por un lado, la necesidad de 
formular nuevos modelos que permitan representar de forma explícita el efecto de las pruebas y mantenimientos sobre dichos criterios RAMS $+C$, ya que estos últimos son utilizados en el proceso de toma de decisiones sobre mejora y optimización de las pruebas y el mantenimiento. En segundo lugar, se requiere desarrollar nuevos métodos de optimización capaces de manejar de forma adecuada tanto la complejidad de los nuevos modelos desarrollados como el gran número de variables de decisión que pueden estar involucradas en el proceso de optimización en base a múltiples criterios RAMS $+C$ y además en presencia de incertidumbres que afectan a la toma de decisiones. Para la resolución del problema de optimización multicriterio que se plantea se ha seleccionado una técnica heurística, concretamente algoritmos genéticos, los cuales han mostrado una alta eficiencia en la resolución de problemas complejos.

\subsection{Objetivos}

El objetivo de la presente tesis se centra en el desarrollo de nuevos modelos y métodos necesarios para acometer el proceso de optimización de pruebas y mantenimientos en base a criterios RAMS $+C$, con aplicación a sistemas de seguridad de centrales nucleares.

La propuesta de los nuevos modelos y métodos requiere de un plan de trabajo en el que en primer lugar se lleve a cabo la investigación del papel de las pruebas y mantenimientos y su efecto en las características RAMS $+C$, de tal manera que su conocimiento permita elaborar los modelos que mejor representen el efecto de los diferentes tipos de pruebas y mantenimientos existentes. A partir de aquí, se requiere desarrollar los métodos de optimización capaces de manejar de forma eficiente la complejidad de los nuevos modelos dentro del proceso de optimización con múltiples variables de decisión y múltiples criterios RAMS $+C$ en presencia de incertidumbres.

\subsection{Resumen de las aportaciones y organización de la tesis}

Como resultado de las diferentes etapas desarrolladas en la tesis se han publicado varios artículos en revistas internacionales incluidas en la base de datos JCR, capítulos de libro, y se ha participado tanto en Congresos Internacionales como Nacionales de elevado prestigio en el área en que se enmarca la presente tesis, como se detalla en el Capítulo 8. Dichas aportaciones ponen de manifiesto que ha existido una evolución dentro del trabajo de investigación hacia la búsqueda de modelos y métodos más realistas. 
A continuación se describen los resultados concretos alcanzados en la tesis. Así, en el Capítulo 5, se proponen varias aproximaciones que modelan el comportamiento de los equipos con independencia temporal, es decir, mediante el uso de valores medios para los atributos RAMS $+C$, que son los más comunes dentro de la metodología de análisis probabilista de riesgos implantada en las centrales nucleares de todo el mundo. Dichos modelos se utilizan posteriormente para la optimización de las pruebas y el mantenimiento de equipos y sistemas en base a criterios RAMS+C. El problema de optimización que se plantea es un problema multiobjetivo sujeto a restricciones que afectan tanto en las variables de decisión como a los criterios adoptados. La resolución de dicho problema se lleva a cabo mediante el uso de Algoritmos Genéticos, Simple Objetivo (AGSO) y Múltiple Objetivo (AGMO), que han sido desarrollados, a partir de los fundamentos de los AA.GG. de última generación propuestos en la literatura.

En la siguiente etapa, se progresa en la dirección de proponer nuevos modelos que tienen en cuenta la variable tiempo en el comportamiento de los equipos desde el punto de vista RAMS $+C$. En este caso se propone una modelización más exacta del efecto de las diferentes estrategias de pruebas y mantenimiento de los equipos integrados a nivel de sistema, que aunque se podían observar utilizando valores medios, sin embargo, ahora permiten además controlar los efectos que dichas estrategias tienen sobre la evolución temporal de los atributos RAMS $+C$. De esta manera se pretende que se puedan cuantificar las fuertes variaciones que ocurren de manera singular en determinados instantes de tiempo como consecuencia de una política de pruebas y mantenimiento no bien ubicada estratégicamente, y al contrario, que basándose en dicha información, el algoritmo de optimización sea capaz de buscar las mejores estrategias. Ello requiere, por un lado adaptar los anteriores AGSO y AGMO para manejar nuevos criterios y variables de decisión, y además integrar mejoras en los algoritmos de optimización que los haga más eficientes.

Por último, en la tercera etapa, se proponen nuevos modelos y métodos que consideran el efecto de las incertidumbres asociadas a los parámetros del modelo, al propio modelo, e incluso a las variables de decisión en el proceso de optimización. La consideración del efecto de las incertidumbres ha planteado tres problemas a resolver: 1) Formulación de las incertidumbres dentro de los modelos RAMS $+C$, 2) Propagación de las incertidumbres para la cuantificación de los atributos RAMS $+C$ y 3) Optimización de las pruebas y el mantenimiento en presencia de incertidumbres.

En primer lugar, se ha utilizado la aproximación probabilista clásica para formular las incertidumbres. En segundo lugar, se ha utilizado Monte Carlo como método de propagación de incertidumbres. Sin embargo, para reducir 
el esfuerzo computacional, se ha combinado el uso del método de Monte Carlo con muestreo puro con el uso de estadísticos de orden. Esta combinación logra obtener límites e intervalos de tolerancia/confianza para la estimación de los atributos RAMS $+C$, que permite tener una precisión suficiente y al mismo tiempo reducir apreciablemente el número de muestras necesarias a generar mediante Monte Carlo. En tercer lugar, se requiere adaptar los anteriores AGSO y AGMO para manejar criterios y variables de decisión inciertos, que son estimados mediante los anteriores límites e intervalos de tolerancia/confianza.

En las tres etapas se aportan casos de aplicación de los nuevos modelos y métodos que demuestran la viabilidad y aplicabilidad de los mismos. 


\section{Vigilancia y mantenimiento}

\subsection{Tipos de vigilancia y mantenimiento}

Las actividades de vigilancia y mantenimiento en las CC.NN. pueden clasificarse en dos grandes grupos: tareas no programadas y programadas. Las tareas no programadas buscan restaurar la capacidad operacional del equipo fallado o degradado (p.e. el mantenimiento correctivo) mientras que las tareas programadas tienen como objetivo controlar la fiabilidad intrínseca del equipo no fallado, por ejemplo, comprobando y controlando que la degradación se encuentre por debajo del punto de fallo (p.e. mantenimiento preventivo).

En este contexto, se define la degradación del equipo como la disminución de sus prestaciones dentro de límites especificados, como consecuencia natural del paso del tiempo (si no se interviene por ejemplo mediante la realización de actividades de mantenimiento) o de la adquisición de edad del equipo, la cual puede resultar en fallo del equipo cuando dicha disminución alcanza en punto límite de fallo definido en sus especificaciones funcionales.

Por otro lado, el fallo del equipo se define como la disminución de su capacidad funcional más allá de sus especificaciones mínimas de funcionamiento, que se puede producir como una interrupción funcional que sobreviene de forma súbita y es posible para cualquier edad del equipo, 0 bien que sobreviene de forma gradual como consecuencia de la acumulación 
de la degradación (rápida, lineal o lenta) que lleva hasta una disminución de prestaciones del equipo por debajo de un umbral establecido en sus especificaciones funcionales.

La Figura 2-1 muestra dos grandes grupos de actividades de mantenimiento, que corresponden con mantenimiento programado y no programado respectivamente.

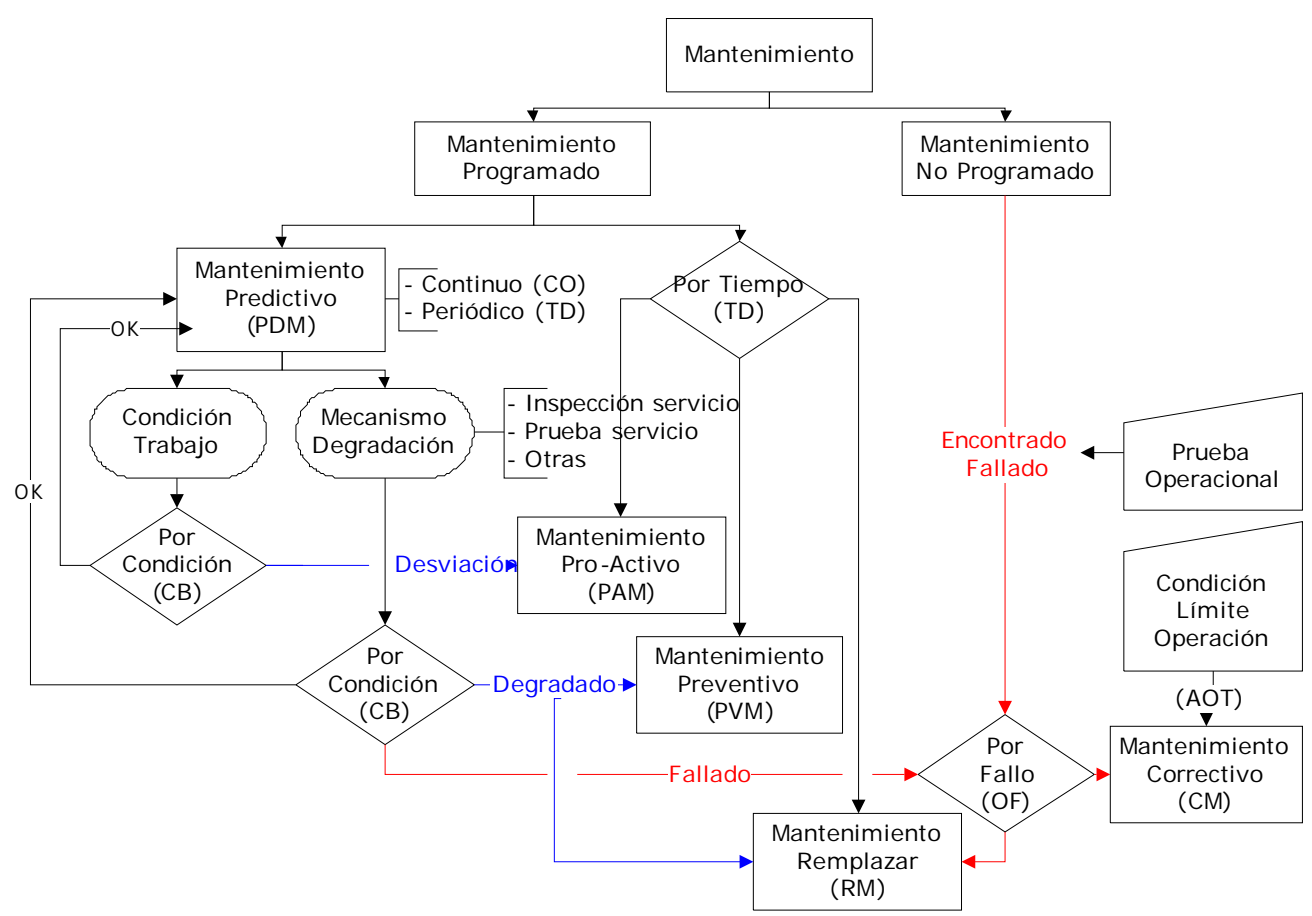

Figura 2-1: Tipos de actividades de pruebas y mantenimientos en las centrales nucleares.

El mantenimiento programado se divide normalmente en dos o más categorías, según se lleven a cabo sobre el equipo en base a tiempo (TD) o por el cumplimiento de alguna condición (CB).

Así pues, el mantenimiento por tiempo representa una actividad que se realiza de acuerdo con una programación preestablecida, por ejemplo, a 
cierta edad del equipo, tras un tiempo cronológico transcurrido, tras un determinado nivel de producción, independientemente de la condición del equipo. Sin embargo, el mantenimiento por condición representa una actividad que se inicia como resultado de que se cumple algún tipo de criterio preestablecido, que se refiere, directa o indirectamente, a la condición funcional del equipo, por ejemplo, atendiendo a que se alcance un cierto estado de degradación del equipo. En este último caso, por tanto, se requiere desarrollar una actividad previa dirigida a evaluar la condición funcional del equipo que se quiere controlar.

La condición funcional del equipo se puede evaluar utilizando técnicas apropiadas al tipo de mecanismo de fallo subyacente, las cuales caen dentro del grupo de actividades denominadas de mantenimiento predictivo (PDM), que se ejecutan mediante monitorización continua ( $\mathrm{CO}$ ) o de forma periódica. EI PDM se centra generalmente en evaluar uno de dos aspectos, las condiciones de servicio, o el efecto del mecanismo de degradación en los materiales o en el funcionamiento del equipo.

La evaluación de las condiciones de servicio se utiliza para decidir si se alcanza la condición para ejecutar un mantenimiento proactivo (PAM), el cual es responsable de controlar que el equipo está trabajando sin desviaciones dentro de las condiciones físico-químicas (por ejemplo, temperatura ambiente, humedad ambiente, temperatura y composición del fluido interno, etc.), tanto internas como externas, especificadas para el equipo, y en cualquier caso lejos del punto de activación del mecanismo de degradación subyacente. Evidentemente, no se requiere ninguna acción proactiva si las condiciones de servicio se evalúan como correctas, como se muestra en la Figura 2-2.

La evaluación del efecto de la degradación, directamente sobre el estado estructural del equipo, por ejemplo, las grietas o desgastes de materiales, o indirectamente sobre parámetros de funcionamiento, por ejemplo, ruido, vibraciones, etc., se utiliza para decidir si se alcanza la condición necesaria para ejecutar un mantenimiento preventivo (PVM) en este caso por condición, que permita mejorar el comportamiento funcional del equipo una vez que se ha puesto de manifiesto el efecto de la degradación. En caso de que la evaluación de la condición funcional del equipo ponga de manifiesto que el estado de degradación del equipo se encuentra cerca del límite de fallo cabe la posibilidad de ejecutar un mantenimiento de sustitución (RM) de parte o todo el equipo, y si el equipo se encuentra fallado (OF) se ejecutará, o bien un mantenimiento correctivo (CM) o bien un reemplazamiento (RM) si dicho fallo corresponde con un estado de degradación del equipo cerca de su límite de fallo. Por el contrario, si el estado de degradación del equipo es aceptable no se ejecutará ninguno de los tipos de mantenimiento anteriores. 


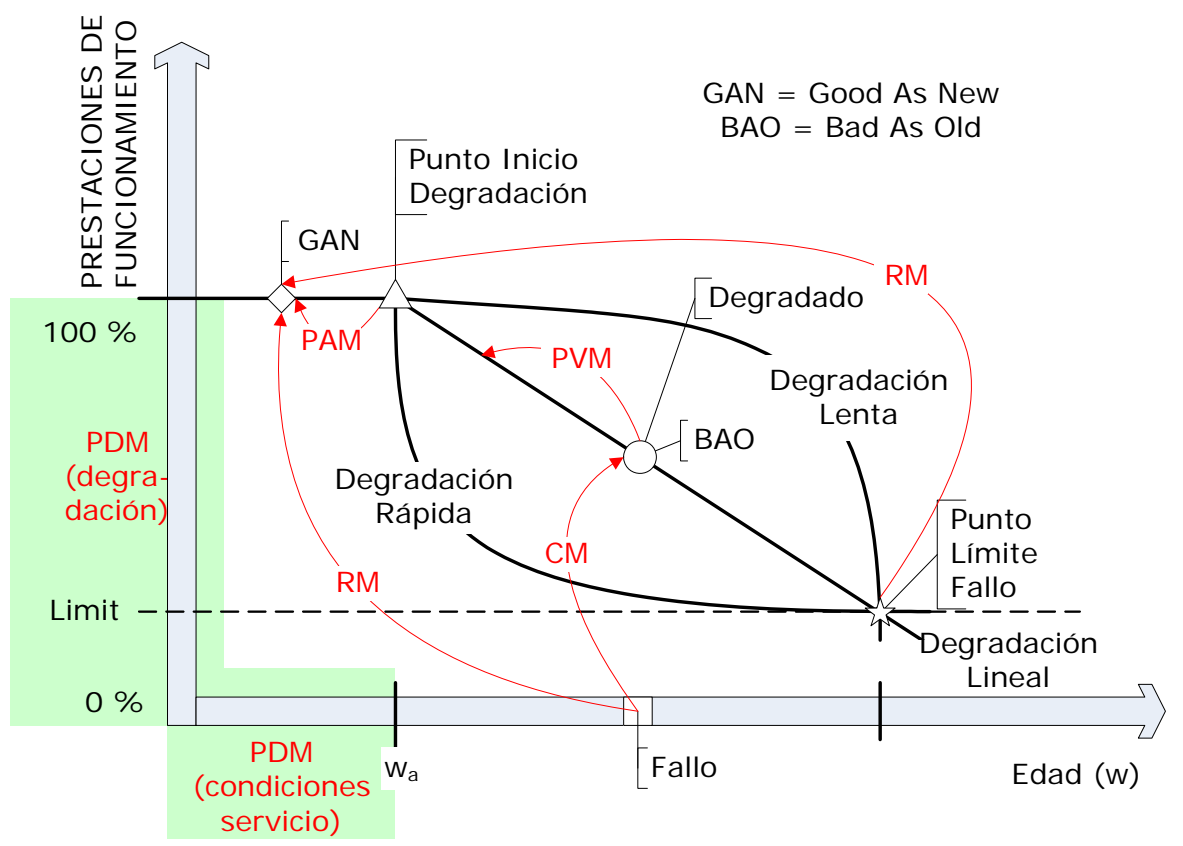

Figura 2-2: Condición funcional del equipo frente a actividades de vigilancia y mantenimiento

El mantenimiento preventivo, PVM, tanto si se realiza en base a tiempo o por condición funcional del equipo, se dirige a revertir el estado de degradación del equipo, llevando al equipo a una situación donde el equipo queda en un estado tan bueno como si fuera nuevo (Good as New, GAN) en caso de ser perfecto, o bien a cualquier estado de degradación intermedio entre la situación actual y dicha situación GAN, que en este caso se dice que se asocia a la ejecución de un mantenimiento imperfecto. Un mantenimiento totalmente imperfecto dejaría al equipo en la misma situación actual (Bad as Old, BAO). El RM y CM representan las dos situaciones extremas de mantenimiento imperfecto respectivamente. Así, en general se adopta que el RM lleva al equipo a una situación GAN, mientras que el CM se encarga de reparar el equipo, es decir, devolviéndolo a un estado de no fallo, pero con capacidad funcional BAO. Tanto el RM como el CM se pueden ejecutar por tiempo en lugar de en base a condición.

Por otro lado, la mayor parte del equipo de seguridad se encuentra 
normalmente en espera (NE), por lo que tanto el CM como el RM, en su caso, se ejecutarán una vez que se ha descubierto que el equipo se encuentra fallado (OF), lo cual resulta posible por dos vías: tras una demanda real del equipo de seguridad a operar, lo cual no resulta obviamente muy deseable, o mediante la realización de una prueba funcional (ST) que se ejecuta de forma periódica por tiempo. En las centrales nucleares dichas pruebas forman parte de las especificaciones técnicas de funcionamiento de la central.

\subsection{Tipos de causas de fallo y su relación con la vigilancia y el mantenimiento}

Esta sección pone de manifiesto la relación que existe entre el comportamiento funcional de equipo, es decir, la evolución de los mecanismos de degradación y las causas de fallo, y las actividades de vigilancia y/o mantenimiento que resultan aplicables de forma individual o combinada para mantener dicho comportamiento dentro de sus límites de funcionamiento normal. La Figura 2-3 muestra esquemáticamente dicha relación.

En lo que se refiere a los fallos originados por degradación, se puede utilizar una actividad planificada por tiempo o por condición, esta última para comprobar la condición según un criterio preestablecido, con el propósito de mantener controlado el fenómeno degradatorio antes de que sobrevenga el fallo de manera imprevista. Cuando la degradación alcanza el punto límite de fallo existe un fallo funcional por cuanto el equipo no tiene prestaciones suficientes para desempeñar su función, como se muestra en la Figura 2-2.

Así, las actividades tipo CO-PDM y TD-PDM, se dirigen a comprobar las condiciones de servicio del equipo o la evolución del mecanismo de degradación. La Figura 2-2 muestra que la degradación no tiene por qué acumularse de manera gradual, es decir, de forma lenta o lineal, por ejemplo, como es el caso del desgaste de componentes como consecuencia de un fenómeno de corrosión. Además, dicha degradación puede darse de forma rápida y sin aviso, por ejemplo, como es el caso tras la posible aparición de cuerpos extraños en una tubería. Por tanto, la monitorización continua de la condición, CO-PDM, es una actividad necesaria para manejar procesos de degradación rápida, mientras que una actividad de mantenimiento predictivo por tiempo, TD-PDM, es suficiente para manejar un fenómeno degradatorio que evoluciona de forma lenta. 


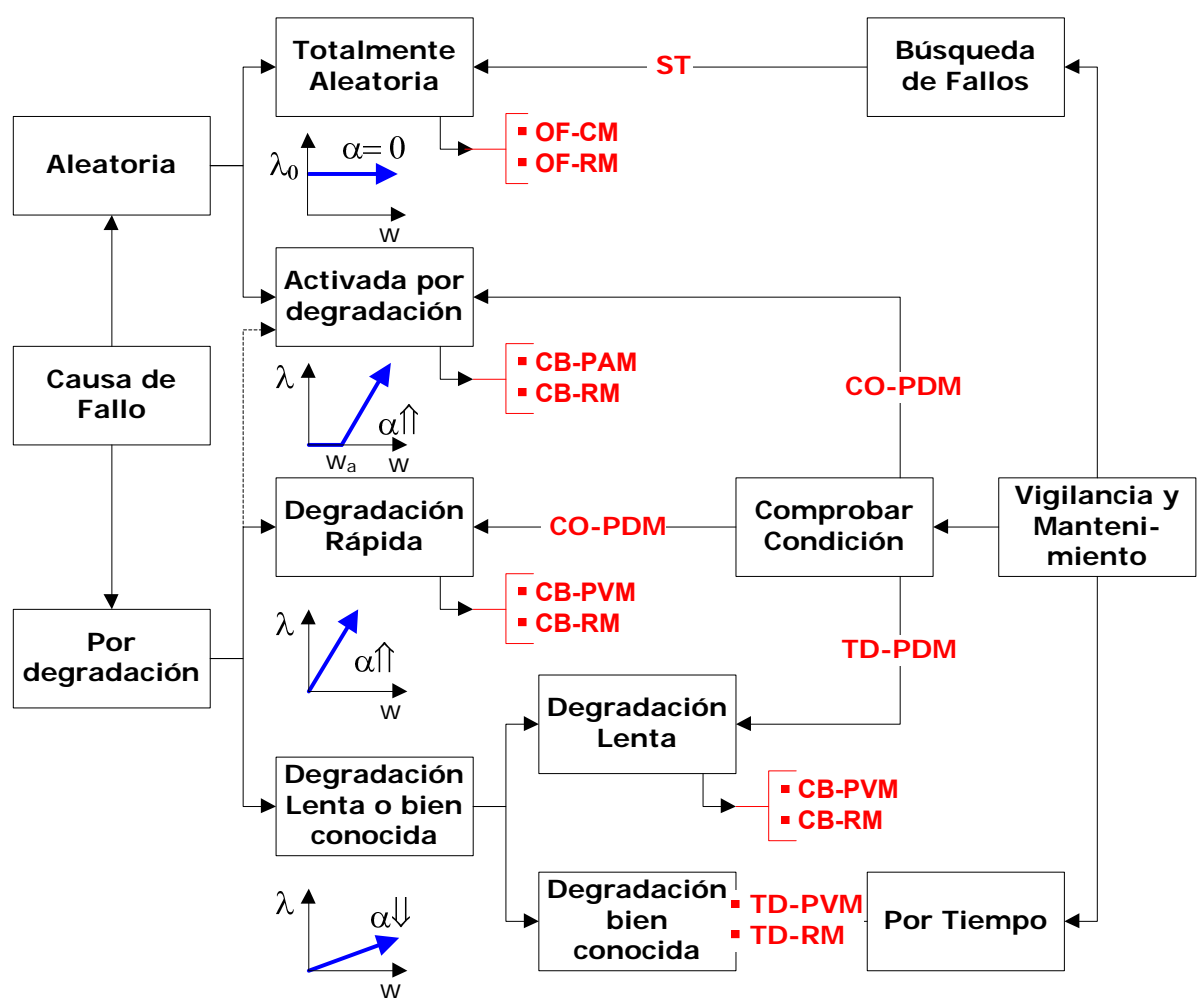

Figura 2-3: Relación entre causas de fallo y las actividades de mantenimiento.

Consecuentemente con lo anterior, una actividad de mantenimiento por condición, bien CB-PVM o bien CB-RM, es necesaria cuando se evalúa, mediante la ejecución de una actividad previa del tipo CO-PDM o TD-PDM, que la degradación del equipo ha alcanzado o superado un nivel preestablecido. Recordar que el mantenimiento de sustitución, RM, deja al equipo en su condición GAN, mientras que el mantenimiento preventivo, PVM, deja al equipo en una condición intermedia entre el GAN and BAO dependiendo de su efectividad, por lo que de forma general se le denomina mantenimiento imperfecto, tal como muestra la Figura 2-2.

De forma similar, las actividades tipo TD-PVM y TD-RM, como se observa en la Figura 2-3, se dirigen a gestionar la evolución del fenómeno de degradación, sin embargo, ahora se ejecutan en base a tiempo y no por condición como las anteriores, por lo que resultan de aplicación para 
controlar fenómenos de degradación que evolucionan de forma bien conocida, por ejemplo tipo lineal.

Por otro lado, el equipo puede ser encontrado fallado tras la realización de cualquier actividad programada. Independientemente de que la causa de fallo sea aleatoria, o no, ésta obliga a ejecutar una actividad no planificada de mantenimiento por fallo, OF, que puede ser del tipo correctivo, OF-CM, o de sustitución si es necesario, OF-RM, para restaurar la operabilidad de equipo supuestamente a una condición BAO, en el caso de CM, o GAN, en el caso del RM.

No obstante, la mayoría de equipos manifiestan un comportamiento caracterizado por una tasa de fallos pequeña y constante a lo largo de la mayor parte de su vida, que se supone de naturaleza aleatoria. Ello se debe a que la mayoría de estos equipos, principalmente activos, son complejos y están constituidos por un gran número de elementos que pueden ser responsable de una o varias causas de fallo, todas ellas caracterizadas por diferentes tasas de fallo. Cuando se consideran de forma conjunta todas las causas de fallo de las piezas del equipo, la tasa de fallos conjunta del equipo se comporta de forma supuestamente constante. Una tasa de fallos constante significa que los fallos sobrevienen de manera aleatoria.

La única protección posible frente a este tipo de fallos de naturaleza puramente aleatoria es mediante la búsqueda de fallos, que se concreta en la realización de pruebas operacionales (ST) para el equipo normalmente en espera y la observación del funcionamiento del equipo normalmente en operación, junto con la realización de actividades OF-CM y OF-RM.

Sin embargo, algunos fallos de naturaleza aleatoria se activan como consecuencia de mecanismos degradatorios que actúan de manera rápida. La protección frente a este tipo de fallos consiste en realizar tareas frecuentes de PDM o CO-PDM en dos líneas. La primera consiste en vigilar las condiciones de servicio del equipo para controlar que se encuentren lejos del punto de activación del fenómeno, como se muestra en la Figura 2-1, realizando CB-PAM en su caso. La segunda consiste en observar la degradación y actuar en su caso mediante CB-PVM y CB-RM. 


\subsection{Requisitos de los modelos de cuantificación RAMS+C adaptados a pruebas y mantenimiento}

Para comprobar el efecto que las distintas actividades de mantenimiento tienen sobre los atributos de $R A M S+C$ se necesita disponer de modelos analíticos que cuantifiquen dichos atributos. Estos modelos han de ser capaces de mostrar explícitamente las relaciones entre los criterios y las variables de interés involucradas en la toma de decisiones.

En particular, los modelos y datos basados en el Análisis Probabilista de Seguridad (APS) se usan frecuentemente para evaluar los criterios RAMS de los equipos relativos a la seguridad (SRE) de las centrales nucleares formulados en términos de parámetros relativos a las pruebas y mantenimientos.

EI APS utiliza modelos analíticos e independientes del tiempo que necesitan extenderse para tener en cuenta explícitamente los efectos de las pruebas y mantenimientos (Martorell 2002).

Además, las contribuciones medias o anuales del coste se obtienen en base a modelos analíticos de dependencia (Harunuzzaman 1996), (Busacca 2001) y (Vinod 2004).

Sin embargo, los modelos numéricos y temporales representan mejor la vida real. Se pueden encontrar muchos ejemplos en la literatura de estos modelos temporales analíticos y numéricos de RAMS+C, (Lapa 2000), (Bunea 2002), (Cepin 2002), (Pereira 2003), (Lapa 2003) y (Lapa 2006). En concreto un modelo de simulación basado en los métodos de Monte Carlo proporciona una herramienta flexible que permite describir muchos de los aspectos relevantes para la optimización de las pruebas y mantenimientos, tales como el envejecimiento, reparaciones imperfectas, obsolescencia, renovación, los cuales no son fácilmente aplicados en modelos analíticos (Borgonovo 2000), (Barata 2002) y (Marseguerra 2002).

Dichos modelos llevan asociados unas incertidumbres, cuyo impacto en los resultados de la optimización de las pruebas y mantenimiento puede ser muy significativo. Por ello, es necesaria una adecuada representación de las incertidumbres en el modelo y en los datos y un procedimiento sistemático para estimar la incertidumbre de los resultados, y cómo la incertidumbre en los parámetros del modelo se propaga en el modelo de decisión basado en criterios RAMS+C (Rocco 2000), (Rocco 2002), (Bunea 2002), (Marseguerra 2004a) y (Coint 2004). Para cuantificar esta incertidumbre a menudo se adoptan representaciones probabilistas y un procedimiento de propagación 
por Monte Carlo con un método de muestreo implementado, $p$, e. Muestreo Simple Aleatorio o Muestreo Latino Hipercúbico.

En esta sección no se intenta proporcionar una presentación comprensiva de todos los modelos disponibles en la literatura para formular todas las posibles relaciones existentes entre causa y efecto introducidas en la sección anterior. En su lugar, la Tabla 2-1 resume los requisitos de modelización relacionados con las actividades de mantenimiento, pruebas, sucesos iniciadores y efectos RAMS asociados con los diferentes tipos de T\&M.

En la Tabla 2-1, los tipos de T\&M representan una de las posibles actividades. La categoría Pre-T\&M está incluida para tener en cuenta que determinadas actividades van precedidas por otra actividad, la cual se ejecuta normalmente para decidir si se llevará a cabo la segunda o no, como muestra la

Figura 2-4. Así por ejemplo, el mantenimiento correctivo por fallo de componentes en espera es llevado a cabo normalmente después de que el componente se encuentre fallado tras una prueba anterior. Sin embargo, el mantenimiento preventivo por tiempo, no requiere de una actividad previa ya que se realiza en base al tiempo transcurrido. El disparador de las actividades de T\&M representa la condición que lanza la segunda actividad, p. e. un condición de fallo del equipo o alcanzar el tiempo límite en los ejemplos anteriores respectivamente, o bien, un mantenimiento preventivo por condición puede empezar una vez que el componente se encuentre en un estado degradado en una actividad previa de mantenimiento predictivo. La categoría Post-T\&M, mostrada en la Tabla 2-1, representa aquellas actividades que siguen a la segunda actividad. Esto es, en el ejemplo del mantenimiento correctivo por fallo, una vez finalizado éste, puede llevarse a cabo una prueba operacional del componente para comprobar su correcto comportamiento. La misma actividad Post-T\&M puede realizarse después de ejecutar un mantenimiento preventivo. La Condición Previa es la condición en la que se encuentra el componente antes de entrar en una actividad determinada. El efecto sobre la fiabilidad (R), disponibilidad (A) y mantenibilidad (M) indica cómo la actividad desarrollada impacta en la fiabilidad, disponibilidad y mantenibilidad del componente. Así, por ejemplo, se sabe si el componente está disponible o fallado después de una prueba, pero la prueba puede también degradar la fiabilidad del componente e imponer una indisponibilidad como consecuencia del tiempo fuera de servicio debido a la misma. 
Tabla 2-1: Resumen de requisitos de modelización de disparo y efectos sobre RAM relacionados con las actividades T\&M.

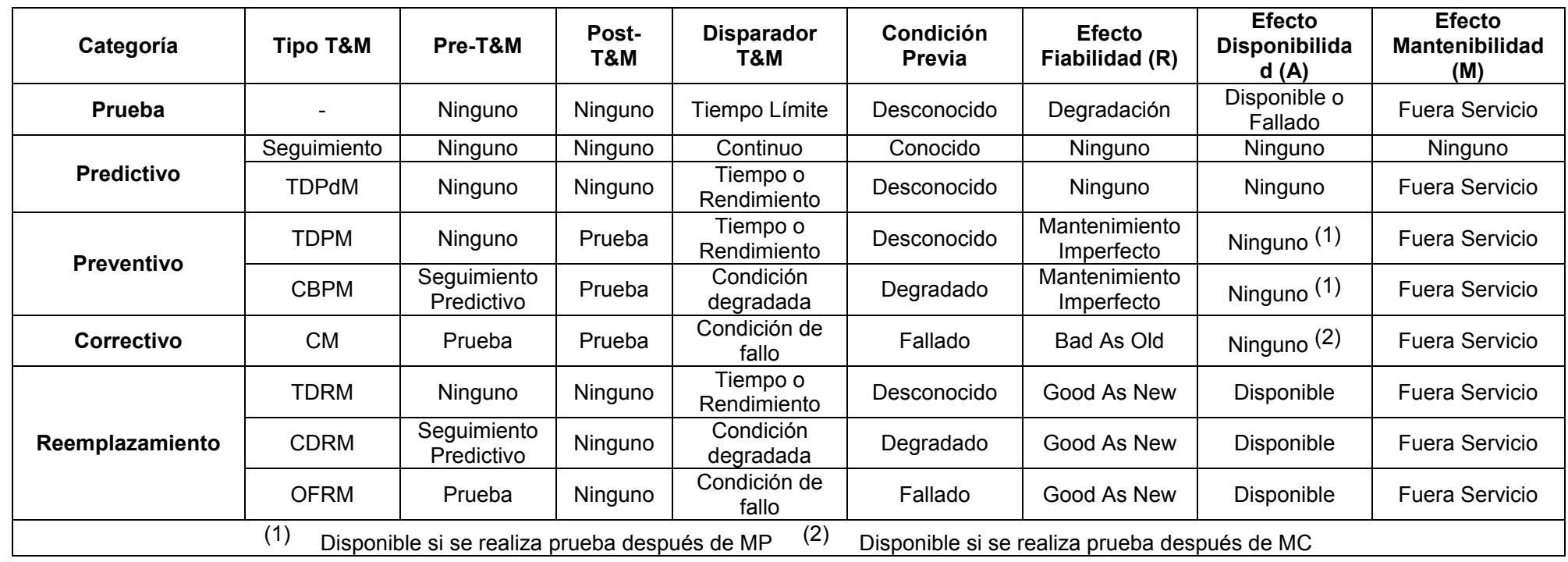




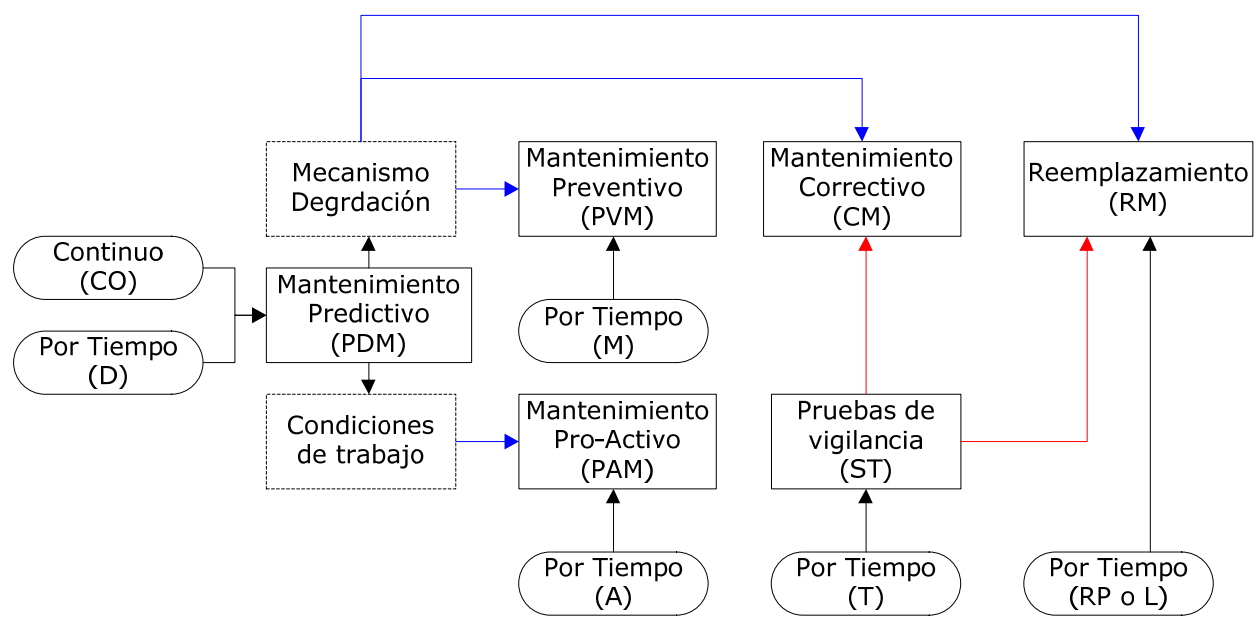

Figura 2-4: Estrategias de mantenimiento por agregación de tareas.

\subsection{Papel de Pruebas, Mantenimientos e Incidencias en criterios RAMS+C}

La Figura 2-5, ilustra los conceptos básicos y el papel de los requisitos incluidos en las Especificaciones Técnicas de Funcionamiento (ETFs) y del Mantenimiento relativos a los atributos RAMS (Fiabilidad, Disponibilidad, Mantenibilidad y Seguridad) de los equipos relativos a la seguridad (SRE) en las centrales nucleares. A continuación se va a detallar cada uno de estos conceptos y su relación entre ellos.

La fiabilidad de un equipo representa su capacidad para responder y mantener la operación sin fallo, es decir, con un comportamiento dentro de sus especificaciones de diseño, bajo condiciones dadas y durante un periodo de tiempo dado o hasta cierta edad. Dentro del concepto de fiabilidad, se pueden diferenciar dos tipos. La fiabilidad natural que es la fiabilidad del equipo $\sin$ mantenimiento que depende directamente de sus características físicas o de su diseño, y la fiabilidad intrínseca que es el valor, en principio superior al natural, obtenido con un mantenimiento normal de calidad, habitualmente mantenimiento preventivo. 


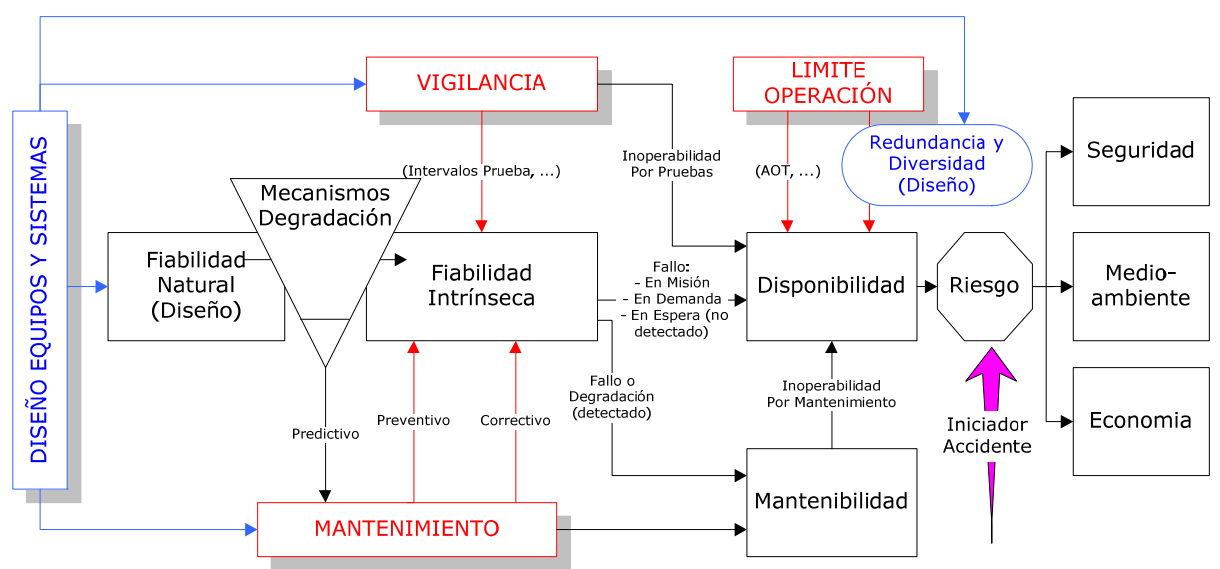

Figura 2-5: Incidencia del diseño, la vigilancia y el mantenimiento en atributos RAMS+C.

El mantenimiento representa todas las actividades realizadas en un equipo con el objetivo de asegurar, mantener o restaurar sus capacidades operacionales. El objetivo del mantenimiento es alcanzar y mantener un nivel implícito de fiabilidad, desde una fiabilidad intrínseca alta, que resulta en necesidades mínimas de mantenimiento, hasta una fiabilidad intrínseca baja que conlleva elevadas demandas de actuaciones de mantenimiento.

El mantenimiento en general introduce dos tipos de efectos positivos. En primer lugar, el mantenimiento correctivo recupera la capacidad operativa del equipo fallado o degradado mientras el mantenimiento preventivo incrementa la fiabilidad intrínseca de los equipos no fallados por encima de la fiabilidad natural, por ejemplo controlando su degradación por debajo del punto de fallo. Aunque el equipo esté sujeto a mantenimiento preventivo y correctivo, puede degradarse por encima de su edad dependiendo de las condiciones de trabajo (operacionales y ambientales) a que esté sometido y de la efectividad del mantenimiento aplicado (mantenimiento imperfecto). Por eso, se programan una serie de actividades para controlar la evolución de los mecanismos de degradación que caen dentro de las categorías de vigilancia continua o mantenimiento predictivo periódico, responsables del lanzamiento de actividades correctivas o preventivas por condición cuando sean necesarias.

Sin embargo, el mantenimiento también introduce efectos adversos, como el efecto del tiempo fuera de servicio (downtime effect) que representa el tiempo 
durante el cual se está realizando una actividad de mantenimiento (correctivo, preventivo, reparaciones, reemplazamientos, puesta a punto, etc.) sobre el equipo encontrándose el mismo inoperable, por lo que este tipo de efecto depende de las características de mantenibilidad del equipo.

Definidos los mantenimientos y las actividades relacionadas que se pueden ejecutar queda más claro el concepto de mantenibilidad que se observa en la Figura 2-5. La mantenibilidad representa la capacidad del equipo o sistema para ser mantenido en unas condiciones específicas durante un periodo de tiempo dado, el cual depende de sus características físicas y de diseño entre otros factores tales como los recursos humanos y los materiales disponibles.

Otro concepto importante es el de disponibilidad del equipo, que representa la capacidad de encontrarse en un estado adecuado para llevar a cabo su función bajo unas condiciones específicas y sin fallar durante un determinado periodo de tiempo. Estar disponible significa no estar fuera de servicio o fallado. Así pues, la disponibilidad, o más directamente la indisponibilidad del equipo no sólo depende del efecto del tiempo fuera de servicio que se vio con anterioridad, sino también de la probabilidad de fallo para llevar a cabo la función para la que fue seleccionado (efecto de no-fiabilidad, unreliability).

A la hora de estudiar la probabilidad de fallo hay que diferenciar los diferentes tipos de fallos. Un fallo puede ocurrir mientras el equipo o el sistema se encuentra desarrollando su función específica (fallo en misión u operación), en el momento de ser requerido para funcionar (fallo a la demanda) o estando en espera para entrar en operación (fallo en espera), este último tipo de fallo está asociado sólo a equipos o sistemas de seguridad, los cuales pueden experimentar fallos en los periodos de espera, que permanecen sin detectar hasta que el equipo es requerido para operar o bien hasta que se le realiza una prueba operacional.

Las especificaciones técnicas de las plantas nucleares a través de los Requisitos de Vigilancia (RV) establecen cómo y cuándo llevar a cabo las pruebas operacionales de los equipos normalmente en espera para limitar el efecto de no-fiabilidad, proporcionando un intervalo entre pruebas (STI) para la realización de las mismas. Las especificaciones técnicas, a través de las Condiciones Límites de Operación (CLO), establecen también el tiempo máximo permitido de inoperabilidad de un equipo con el objetivo de limitar el efecto del tiempo fuera de servicio.

En particular, durante las últimas décadas se ha prestado mucha atención a los requisitos de vigilancia y a las condiciones límite de operación en las aplicaciones informadas en el riesgo (risk-informed) de los Análisis 
Probabilista de Seguridad de determinadas plantas nucleares para apoyar cambios en dichos requisitos.

En la Figura 2-6, se muestra el contenido típico de las Condiciones Límite de Operación y de los Requisitos de Vigilancia incluidos en las especificaciones técnicas usuales.

Para ilustrar los fundamentos mostrados en este apartado tomemos como ejemplo las CLO y los RV de un sistema de seguridad concreto como pueden ser las fuentes de corriente alterna de una planta nuclear tipo Westinghouse en operación normal, ETF 3/4.8.1 (NUREG1431). En particular se ha escogido para guiar la explicación los generadores diesel de emergencia.

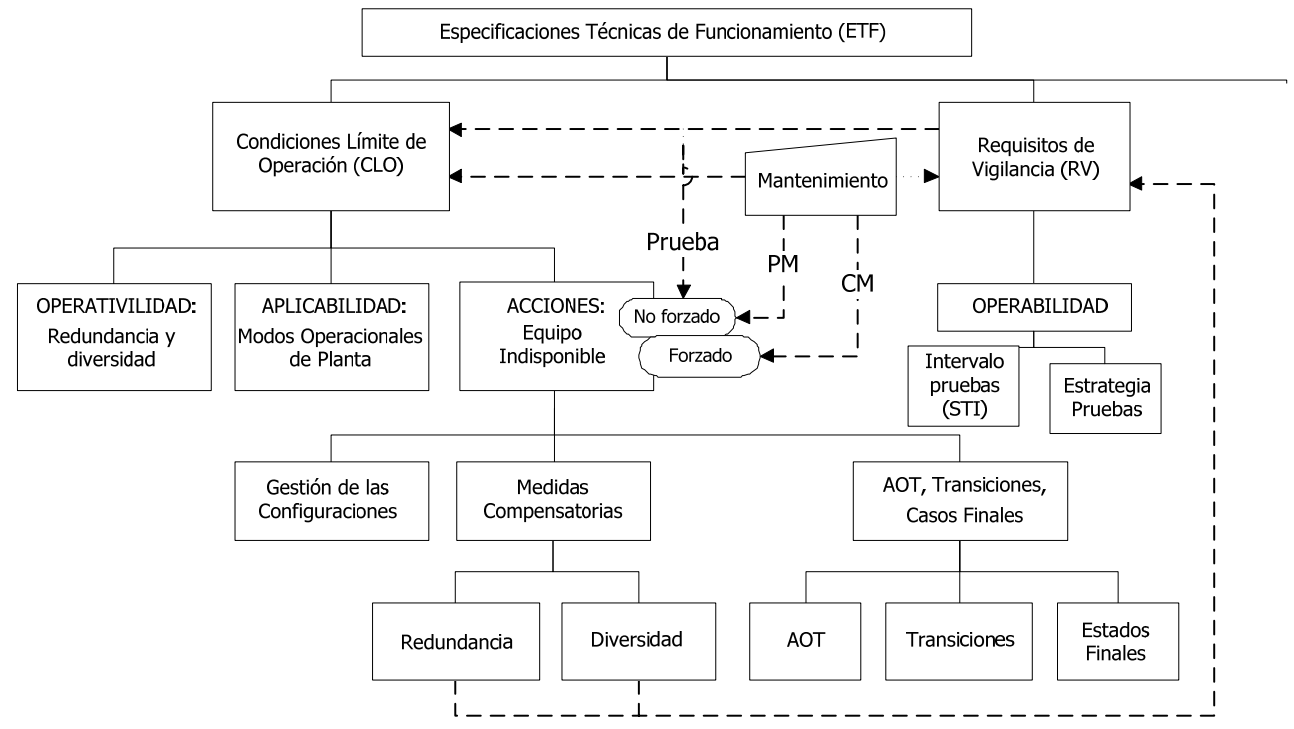

Figura 2-6: Pruebas y mantenimiento en relación a las ETFs de las centrales nucleares.

Las CLO son las capacidades funcionales o niveles de funcionamiento más bajos requeridos a un equipo para la operación segura de una planta nuclear, y en ellas se especifican la operabilidad de los sistemas a los que hace referencia; la aplicabilidad en el contexto de cuándo son de aplicación dichas CLO, es decir, a qué modos de operación aplican; y las acciones a llevar a cabo en caso de alcanzar dichas condiciones límite. Así, para el ejemplo de la aplicación, la CLO establece la operabilidad de las fuentes de 
corriente alterna redundantes de diversa naturaleza (suministro exterior, generadores diésel de emergencia, etc.) aplicable en todos los modos de operación de una central nuclear, desde operación a plena potencia hasta parada. La redundancia de fuentes de corriente alterna se consigue mediante dos generadores diésel de emergencia, físicamente independientes y capaces de suministrar la energía eléctrica demandada a los sistemas.

Cuando una CLO de una planta nuclear no se cumple, el titular de la licencia de dicha instalación deberá parar el reactor o seguir cualquier acción permitida por las Especificaciones Técnicas hasta que la condición vuelva a ser restablecida. Así, por ejemplo, con un generador diesel de emergencia indisponible se entra en una configuración de riesgo y se plantea la introducción de medidas compensatorias, tales como demostrar la operabilidad de una fuente de alimentación externa y la operabilidad del tren redundante de generadores diesel mediante una prueba de vigilancia. Además, la CLO establece que el generador diesel de emergencia indisponible debe ser restaurado a una condición de operabilidad antes de un tiempo determinado, tiempo que representa el máximo tiempo permitido fuera de servicio o AOT, o de otra forma se requerirá llevar a parada la central mediante una transición ordenada (operación a potencia, parada caliente, parada fría) a seguir y un tiempo disponible para alcanzar un estado final más seguro.

Los RV establecen las pruebas de vigilancia periódicas a realizar para la detección de fallos, verificando la operabilidad de los sistemas de seguridad. Los RV definen el tipo de prueba de vigilancia, la estrategia de pruebas y la frecuencia, estableciendo el intervalo de tiempo entre dos pruebas de vigilancia consecutivas. Por ejemplo, en el caso de los generadores diesel de emergencia uno de dichos requisitos establece un intervalo entre pruebas (STI) de 31 días para el generador diesel de emergencia con una frecuencia escalonada (se prueba uno de los trenes de los generadores diesel de emergencia cada quince días).

Finalmente, tras el ejemplo anterior, el último de los atributos, el atributo de seguridad de estos sistemas referentes a la seguridad de la planta, puede ser definido como su capacidad para prevenir o mitigar las consecuencias de accidentes postulados, llamados Accidentes Base de Diseño (DBA, design based accidents) en los Análisis Finales de Seguridad (AFS) de las centrales, los cuales podrían causar un riesgo excesivo a la salud y a la seguridad del público. Por ello, el riesgo es a menudo adoptado como medida de la seguridad de las centrales nucleares, y puede ser entonces definido como la probabilidad de causar un daño a la salud y a la seguridad del público. Además, los riesgos medioambientales y financieros pueden ser considerados también como dependientes de forma parcial de los atributos 
RAM de dichos sistemas.

Como se aprecia en la Figura 2-5, la seguridad de las centrales nucleares se basa en los principios de redundancia, diversidad $y$ disponibilidad (fiabilidad) de sus SRE. Es por ello, que en general los sistemas de seguridad consisten en un número de trenes redundantes para llevar a cabo dichas funciones de seguridad. La seguridad también se basa en la diversidad e independencia de los SRE para llevar a cabo tales funciones de seguridad; incluso más, diferentes trenes del mismo sistema son de diferente naturaleza en algunos casos. Consecuentemente, el riesgo de las centrales nucleares aumenta con la perdida de redundancia e indisponibilidad (no fiabilidad) de sus SRE.

\subsection{Programas para la mejora de Pruebas y Mantenimiento.}

En el Capítulo 1, se ha hecho un breve planteamiento de la situación actual de las centrales nucleares que muestra cómo diferentes programas implementados en la planta ayudan a la mejora de parámetros de las especificaciones técnicas y del mantenimiento. De acuerdo con los objetivos establecidos en estos programas, es posible dividirlos en dos grupos diferentes, unos centrados en la mejora de las bases de licenciamiento y otros centrados en la mejora de las actividades de mantenimiento, tal como se ve en la Figura 2-7.

En el primer grupo se incluyen los programas de bases de licenciamiento informadas en el riesgo (RI-LB, risk informed licensing basis), centrados en la mejora de parámetros relativos a las especificaciones técnicas de funcionamiento (RI-TS, risk informed technical specification), a inspecciones (RI-ISI, risk informed in service inspection) y a pruebas (RI-IST, risk informed in service test). Estos programas trabajan en términos de fiabilidad (RI-SR), mantenibilidad (RI-SR en lo referente a indisponibilidad debida a pruebas y RI-LCO en lo referente a indisponibilidad debido a mantenimiento) y seguridad (RI-LB en general en lo referente al riesgo condicionado, riesgo simple y la contribución al riesgo anual de la planta).

El segundo grupo de programas se centran en la mejora de la política de mantenimiento. Dentro de este grupo nos encontramos con programas cuyo objetivo se centra en mantener una alta fiabilidad intrínseca, reduciendo tanto como sea posible la tasa de fallo (Mantenimiento Centrado en la Fiabilidad, RCM). Otros programas buscan un control sobre la degradación de los diferentes sistemas que afectan a la fiabilidad de éstos (Programa de Gestión de Vida, LMP). Relacionados con los anteriores programas han 
surgido otros más específicos enfocados, por ejemplo, con la renovación de la licencia de operación (Aplicación de Renovación de Licencia, LRA).

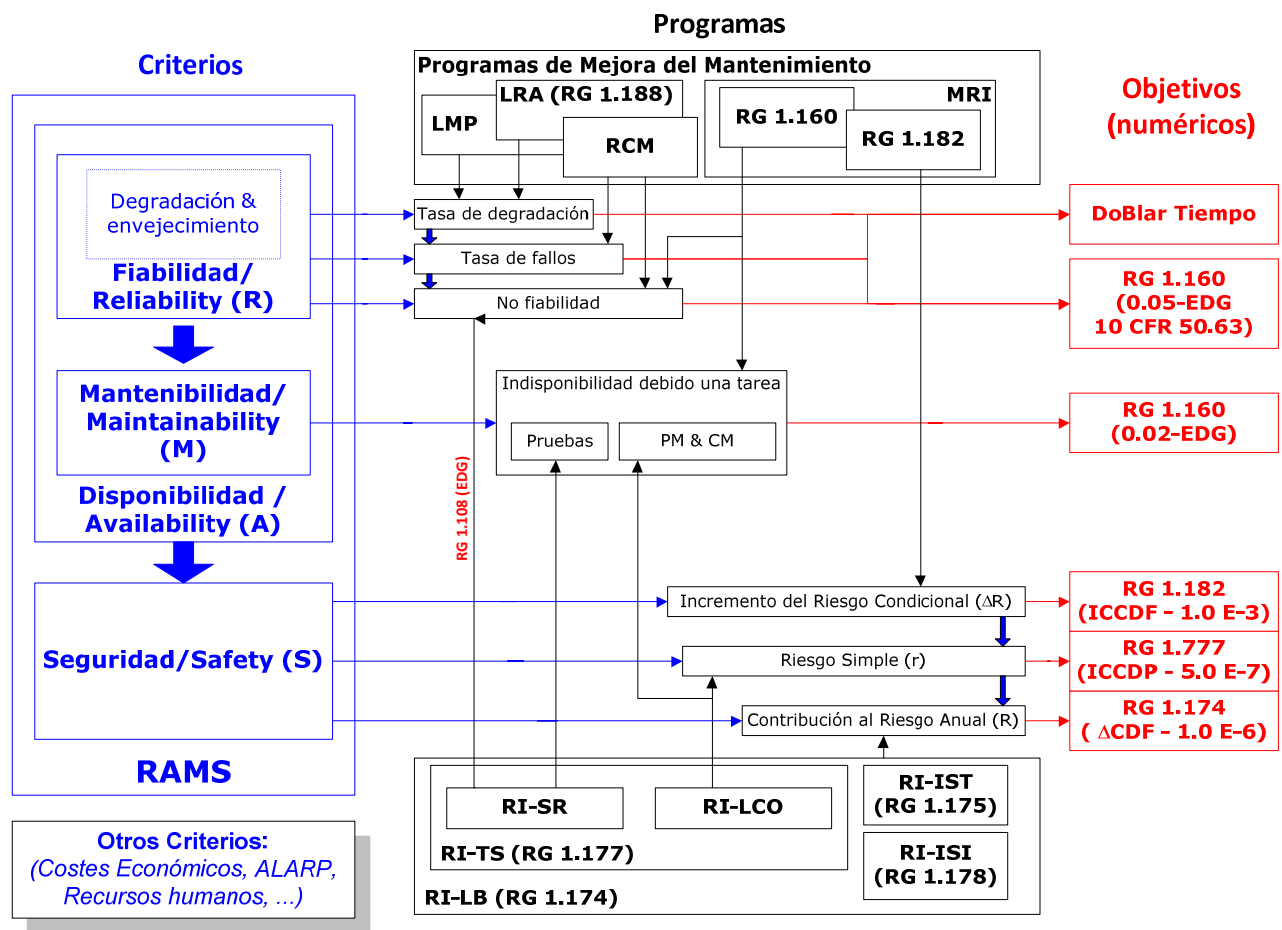

Figura 2-7: Programas implementados para la mejora de ETFs y mantenimiento en Centrales Nucleares.

En la misma línea que estos programas cuyo objetivo se centra en una mejora de la fiabilidad de los equipos, existen programas que buscan además mantener una alta mantenibilidad al mismo tiempo, teniendo en cuenta los efectos opuestos que una alta fiabilidad puede tener sobre la mantenibilidad (Programa de Implementación de la Regla de Mantenimiento, MRI).

Para todos estos programas existen una serie de objetivos numéricos para limitar el efecto que los posibles cambios pueden tener en especial sobre la salud y la seguridad de la población y que viene dados por las guías reguladoras (RG 1174) y (RG 1177). 



\section{Modelado de parámetros RAMS+C}

\subsection{Modelo de fiabilidad}

La fiabilidad de un equipo representa su capacidad para responder y mantener la operación sin fallo, es decir, con un comportamiento dentro de sus especificaciones de diseño, bajo condiciones dadas y durante un periodo de tiempo determinado o hasta una cierta edad. La fiabilidad natural es la fiabilidad del equipo no sometida a ninguna actividad de mantenimiento, la cual depende exclusivamente de sus características físicas o de diseño, mientras que la fiabilidad intrínseca es el valor de fiabilidad, en principio superior al valor natural, logrado con un mantenimiento de calidad, normalmente programado.

La edad de un componente se define como el periodo de tiempo transcurrido desde su instalación, teniendo en cuenta los efectos que las condiciones de trabajo y las actividades de mantenimiento a las que esté sometido tienen sobre su vida útil. Las condiciones de servicio de un componente se dividen básicamente en dos: operacionales y ambientales. Las condiciones operacionales reflejan su modo de operación, es decir si opera continua o intermitentemente, a plena carga o carga parcial, etc. Por otra parte las 
condiciones ambientales reflejan parámetros físicos del medio en que se encuentra ubicado el equipo, tales como la presión, temperatura, humedad, voltaje etc...

El otro efecto a considerar en la edad del equipo es el introducido por la realización de las tareas de pruebas y mantenimiento y que afectan a la edad del componente. En general, se puede asumir que cada actividad de mantenimiento mejora la edad del componente en cierto grado, dependiendo de su efectividad, esto se conoce como mantenimiento imperfecto, que es una generalización natural de dos situaciones extremas. Dichas situaciones extremas suponen por una parte considerar que tras el mantenimiento el equipo queda en un estado tan bueno como si fuera nuevo (Good As New, GAN), lo cual significa que su edad ha sido restaurada a cero después de llevar a cabo el mantenimiento (efectividad del mantenimiento igual a uno). Por otro lado, la segunda opción es asumir que el mantenimiento deja al componente en un estado como el anterior a la realización del mismo (Bad As Old, BAO), lo cual significa que la edad del componente tras el mantenimiento es la misma que inmediatamente antes de realizar dicha actividad (efectividad del mantenimiento igual a cero).

La evolución de la edad en el periodo $m$ entre dos mantenimientos consecutivos, $m-1$ y $m$, viene dada por la expresión:

$\mathrm{w}_{\mathrm{m}}(\mathrm{t}, \varepsilon, \mathrm{z})=\mathrm{w}_{\mathrm{m}-1}^{+}+\Psi(\mathrm{z})\left(\mathrm{t}-\mathrm{t}_{\mathrm{m}-1}\right)$

Donde $w_{m-1}^{+}$representa la edad del componente inmediatamente después del mantenimiento $m-1$ y $\Psi(z)$ la función vínculo que introduce la influencia de las condiciones de servicio en el periodo $m$.

Existen varios modelos desarrollados para simular el mantenimiento imperfecto (Martorell 1996), de los cuales se van a considerar dos modelos los cuales asumen que cada actividad de mantenimiento reduce la edad del componente desde el punto de vista de la tasa de ocurrencia de fallos. Estos modelos son el modelo PAR (Proportional Age Reduction) y PAS (Proportional Age Setback) (Martorell 1999b).

Estos modelos se basan en los siguientes supuestos:

$\checkmark \quad$ La efectividad de mantenimiento se representa por un parámetro, $\varepsilon$, en el intervalo $[0,1]$ 
$\checkmark \quad$ Las condiciones de trabajo (ambientales y operacionales) se suponen constantes y normales.

En las siguientes secciones se describen detalladamente ambos modelos.

\subsubsection{Modelo PAS.}

En la aproximación PAS, se asume que cada actividad de mantenimiento reduce proporcionalmente a su efectividad, $\varepsilon$, la edad que el componente tenía inmediatamente antes de dicho mantenimiento.

Si $\varepsilon=0$, el modelo PAS se reduce a una situación $\mathrm{BAO}$, mientras que si $\varepsilon=1$ el modelo reproduce una situación GAN. Así, este modelo es una generalización natural de los modelos GAN y BAO.

Si se adopta este modelo la edad del componente inmediatamente después de la actividad $(m-1)$ de mantenimiento, $w_{m-1}^{+}$, viene dada por (Martorell 1999b):

$\mathrm{w}_{\mathrm{m}-1}^{+}=\mathrm{t}_{\mathrm{m}-1}-\sum_{\mathrm{k}=0}^{\mathrm{m}-2}(1-\varepsilon)^{\mathrm{k}} \cdot \varepsilon \cdot \mathrm{t}_{\mathrm{m}-\mathrm{k}-1}$

donde $t_{m-1}$ es el tiempo en el que tuvo lugar la actividad $m-1$ de mantenimiento.

A partir de la ecuación (3-2) es posible obtener un modelo de fiabilidad dependiente de la edad en donde la tasa de fallos inducida o condicional, $\lambda_{m}(w)$, en el periodo $m$, después de la actividad de mantenimiento $m-1$ viene dada por:

$\lambda_{\mathrm{m}}(\mathrm{w})=\lambda(\mathrm{w}(\mathrm{t}, \varepsilon))+\lambda_{0} \quad \mathrm{w} \geq \mathrm{w}_{\mathrm{m}-1}^{+}$

donde $\lambda_{0}$ representa la tasa de fallos inicial del componente, es decir, en el momento en el que fue instalado.

Considerando que la edad del componente después del mantenimiento $m-1$ 
viene dada por la ecuación (3-2), y adoptando un modelo lineal para la tasa de fallos, la expresión para la tasa de fallos inducida o condicional después del mantenimiento $m-1$ se puede escribir como:

$\lambda_{\mathrm{m}}(\mathrm{w})=\{\alpha \cdot \mathrm{w}(\mathrm{t}, \mathrm{\varepsilon})\}+\lambda_{0} \quad \mathrm{w} \geq \mathrm{w}_{\mathrm{m}-1}^{+}$

donde $\alpha$ es la tasa de envejecimiento lineal.

Sustituyendo las ecuaciones (3-2) y (3-4) en la ecuación (3-1), y asumiendo condiciones normales de trabajo, es decir $\Psi(z)=1$, la edad del componente se expresa como:

$\mathrm{w}(\mathrm{t}, \varepsilon)=\mathrm{w}_{\mathrm{m}-1}^{+}+\left(\mathrm{t}-\mathrm{t}_{\mathrm{m}-1}\right) \quad \mathrm{w} \geq \mathrm{w}_{\mathrm{m}-1}^{+}$

donde $t$ es tiempo cronológico desde el origen, y $t_{m-1}$ es el tiempo en el que se llevó a cabo la actividad de mantenimiento $m-1$.

Se puede calcular una tasa de fallos en espera media en el periodo de reemplazamiento, basada en un proceso de doble promedio (Martorell 1999b). Con lo que la expresión final para el modelo de tasa de fallos con mantenimiento imperfecto bajo la aproximación PAS está dada por

$\lambda=\lambda_{0}+\frac{\alpha}{2 \cdot \mathrm{f}} \cdot \frac{2-\varepsilon}{\varepsilon}$

donde a cada tarea de mantenimiento, ya sea programada o no, se le puede asociar una frecuencia, $f$, y una efectividad, $\varepsilon$.

\subsubsection{Modelo PAR.}

En la aproximación PAR, cada actividad de mantenimiento se asume que reduce proporcionalmente la edad ganada desde la última actividad de mantenimiento. Así, mientras que en la aproximación PAS se considera que cada actividad de mantenimiento reduce la edad total de componente, el modelo PAR, explicado con detalle en (Martorell 1999b), considera que el mantenimiento reduce la edad ganada entre dos actividades consecutivas en un factor, $\varepsilon$, efectividad del mantenimiento, que toma valores en el intervalo $[0,1]$. Análogamente al modelo PAS se puede observar que si $\varepsilon=0$ el modelo PAR reproduce el modelo $\mathrm{BAO}$, mientras que si $\varepsilon=1$ representa 
unas condiciones GAN.

De forma similar al desarrollo presentado para el modelo PAS, se puede evaluar la edad del componente inmediatamente después de la actividad de mantenimiento $m-1$ que, utilizando el modelo PAR, viene dada por:

$\mathrm{w}_{\mathrm{m}-1}^{+}=(1-\varepsilon) \cdot \mathrm{t}_{\mathrm{m}-1}$

donde $t_{m-1}$ es el tiempo en el cual se lleva a cabo el mantenimiento $m-1$, y $\varepsilon$ es la efectividad del mismo.

Usando un proceso similar al descrito para el modelo PAS es posible derivar la expresión del modelo de tasa de fallo para mantenimiento imperfecto bajo la aproximación PAR:

$\lambda=\lambda_{0}+\frac{\alpha}{2 \cdot f}[1+(1-\varepsilon) \cdot(f \cdot R P-1)]$

donde $R P$ es el periodo de reemplazamiento, $f$ la frecuencia del mantenimiento, y $\varepsilon$ la efectividad del mismo.

\subsection{Efecto de las actividades de vigilancia y mantenimiento sobre los atributos RAM}

Así, atendiendo a los efectos sobre RAM relacionados con las actividades de pruebas y mantenimientos según la Tabla 2-1 y atendiendo a la Figura 3-1, por ejemplo, una prueba que se ejecuta con un tiempo límite $T$, tiene asociado una frecuencia $f=1 / T$ y una $\varepsilon=0$ (BAO). Los mantenimientos, como el mantenimiento correctivo por fallo, lleva asociado una frecuencia $f=$ $\lambda$ y una $\varepsilon=0$ (BAO), un reemplazamiento por tiempo (a un tiempo $R P$ ), una frecuencia $f=1 / R P$ y una efectividad $\varepsilon=1$ (GAN), y un mantenimiento preventivo por tiempo (a un tiempo $M$ ), una frecuencia $f=1 / M$ y una $\varepsilon$ entre ]0,1] (mantenimiento imperfecto). Además, un mantenimiento predictivo por tiempo (a un tiempo $D$ ) lleva asociado una $f=1 / D$ y una $\varepsilon=0$, mientras que el mantenimiento preventivo por condición se asocia a una $f=P d / D$ y una $\varepsilon$ dentro del rango 10,1$]$, siendo $P d$ la probabilidad de encontrar al equipo degradado después de un mantenimiento predictivo. También, un mantenimiento proactivo por tiempo (a un tiempo $A$ ) se le puede asociar una $f$ $=1 / A$ y una $\varepsilon=1$. 


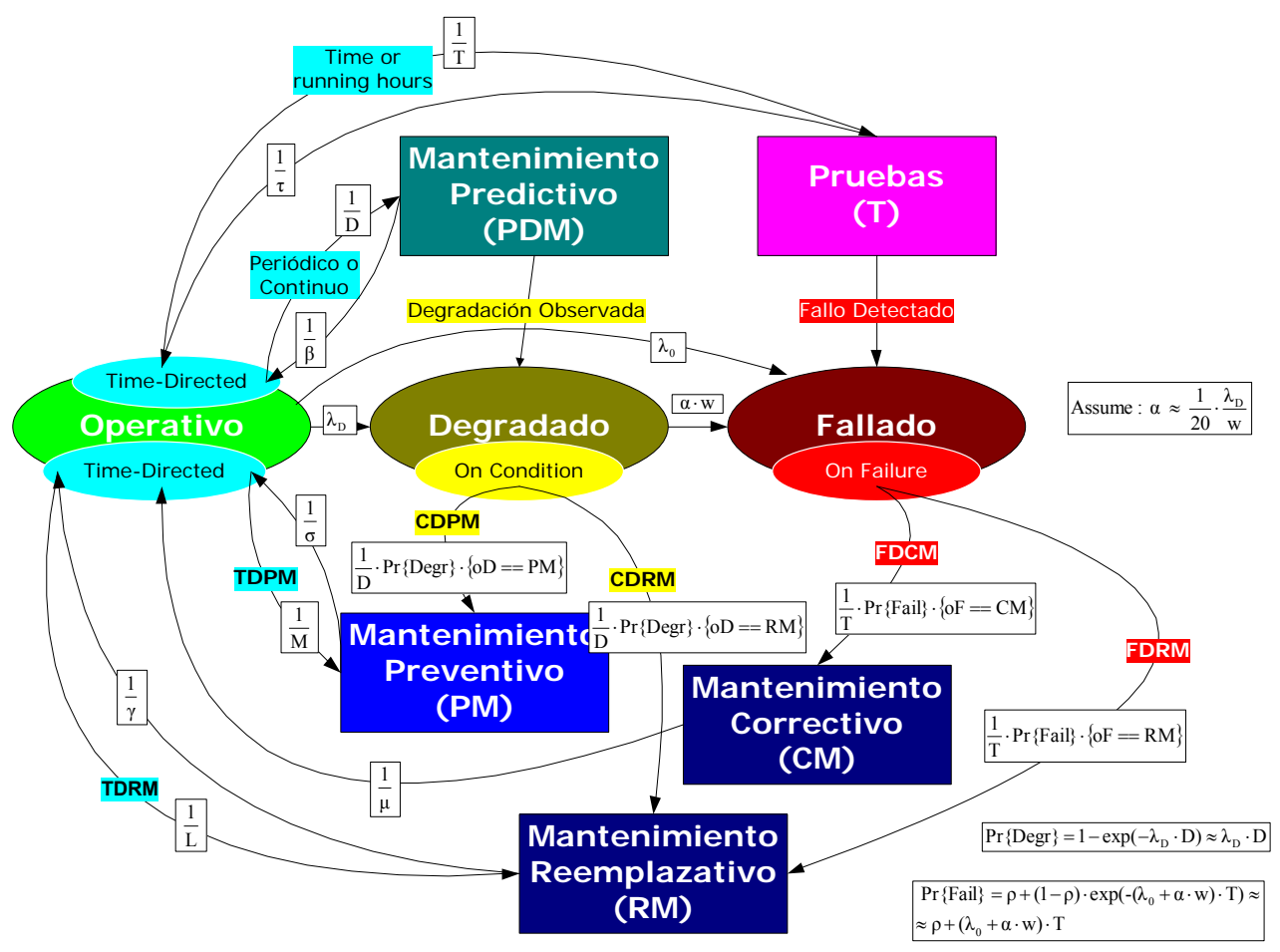

Figura 3-1: Diagrama de estados funcionales, actividades de mantenimiento, pruebas y transiciones entre equipos de seguridad.

\subsection{Modelos de mantenibilidad}

El mantenimiento introduce al menos dos tipos de aspectos positivos. Primero, el mantenimiento correctivo restaura la capacidad funcional del equipo fallado o degradado y el mantenimiento programado incrementa su fiabilidad intrínseca de los equipos no fallados por encima de su fiabilidad natural, por ejemplo, controlando la evolución de su degradación para que se encuentre siempre por debajo del punto de fallo. Sin embargo, el hecho de que un equipo esté sometido a un programa de mantenimiento programado y correctivo no impide que el equipo pueda degradarse con el paso del tiempo como consecuencia de sus condiciones de servicio, es decir, condiciones operacionales y ambientales, y como consecuencia de un mantenimiento imperfecto, es decir, un mantenimiento que no sea completamente eficaz. Así, existen diversas actividades programadas de mantenimiento para valorar 
la evolución de los mecanismos de degradación principales, las cuales incluyen monitorización continua y mantenimiento predictivo periódico y son responsables de lanzar otras actividades de mantenimiento preventivo por condición y correctivo cuando resulte necesario para controlar la evolución de la degradación. La fiabilidad del equipo se ve modificada por el mantenimiento que se le realiza.

Por el contrario, el mantenimiento también introduce efectos adversos como consecuencia de la inoperabilidad forzada del equipo durante el tiempo que éste se encuentra fuera de servicio sometido a mantenimiento (correctivo, preventivo, sustitución, etc.). Además, la importancia de dicho efecto adverso depende de las características de mantenibilidad del equipo.

La mantenibilidad representa la capacidad de un equipo para ser mantenido bajo condiciones específicas durante un periodo de tiempo dado, el cual depende no sólo de las características físicas del equipo imponiendo un número dado de horas-hombre $(H)$ para llevar a cabo una tarea individual de mantenimiento, sino también de los recursos humanos y materiales disponibles para llevar a cabo dicha tarea, tiempos de retraso, etc., los cuales influyen en el tiempo real que el equipo está fuera de servicio $(d)$ para realizar una tarea concreta de mantenimiento. La Figura 3-2 muestra un esquema de los tiempos invertidos en la realización de un determinado mantenimiento.

A partir de la Figura 3-2, el tiempo fuera de servicio, $d$, se puede estimar para una tarea de mantenimiento como sigue:

$\mathrm{d}=\mathrm{T}_{\mathrm{D}}+\frac{\mathrm{H}}{\left(\eta_{\mathrm{P}} \cdot \mathrm{N}_{\mathrm{P}}+\eta_{\mathrm{E}} \cdot \mathrm{N}_{\mathrm{E}}\right) \cdot \kappa\left[\mathrm{N}_{\mathrm{P}}+\mathrm{N}_{\mathrm{E}}\right]}$

siendo $T_{D}$ el máximo entre el tiempo de demora del material y el tiempo de demora de los recursos humanos y $\mathrm{H}$ el número necesario de horas-hombre para la realización de la tarea. $N_{P}$ y $N_{E}$ representan el número de personal propio y externo que intervienen en dicha tarea, respectivamente, los cuales tienen asociados unas eficiencias en la ejecución la misma, representadas por $\eta_{P}$ y $\eta_{E}$. Por último, la función $k[$ [.] representa la ley de decrecimiento de la efectividad a medida que aumenta el número de personal tanto propio como externo involucrado en la realización de la actividad que puede ser formulada como:

$\kappa[N]=\exp \left[K \cdot\left(-1+\frac{1}{N}\right)\right]$ 


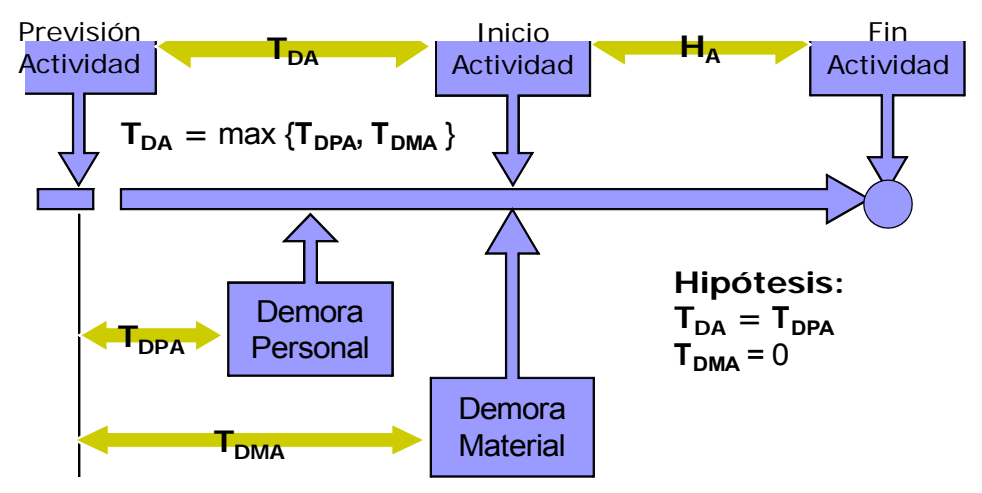

Figura 3-2: Esquema de la mantenibilidad de un equipo para una tarea A.

siendo $N$ el número total de personal, $N_{p}+N_{E}$. Según la expresión (3-10) $K$ varía en el intervalo $[0,1]$.

En general, la ecuación (3-9) se simplifica en el caso de actividades programadas por tiempo ya que el tiempo de demora $T_{D}$ es nulo.

Para la mayoría de las aplicaciones se suele asumir un término medio en el tiempo de realización en función de la experiencia operacional.

\subsection{Modelos de disponibilidad}

Cada causa de fallo del equipo debe estar cubierta por una tarea de mantenimiento. Una pareja de causa de fallo y tarea de mantenimiento lleva asociada una cierta indisponibilidad del equipo. Dicha indisponibilidad corresponderá al menos con una de las contribuciones siguientes (Martorell 2002):

$\mathrm{u}_{\mathrm{r}}(\mathrm{x})=\left(1-\frac{1}{\lambda \cdot \mathrm{I}} \cdot\left(1-\mathrm{e}^{-\lambda \cdot \mathrm{I}}\right)\right) \approx \rho+\frac{1}{2} \lambda \cdot \mathrm{I}$

$\mathrm{u}_{\mathrm{S}}=\mathrm{f}_{\mathrm{S}} \cdot \mathrm{d}_{\mathrm{S}}$ 
$u_{N}=f_{N} \cdot d_{N} \cdot G[A O T]$

La ecuación (3-11) representa la indisponibilidad asociada con un fallo nodetectado, donde $I$ es el intervalo para ejecutar una tarea de mantenimiento programado cuyo objetivo es detectar la ocurrencia de dicho fallo, $\lambda$ es la tasa de fallo la cual puede ser evaluada a partir de las ecuaciones (3-6) y (3-8) en función del modelo de edad seleccionado y $\rho$ representa la probabilidad de fallo a la demanda del equipo.

La ecuación (3-12) corresponde a la contribución a la indisponibilidad asociada a una tarea de mantenimiento o prueba planificada o programada, donde $f_{S}$ representa la frecuencia de dicha actividad dada por $f_{S}=1 / /$, mientras que $d_{S}$ es el tiempo fuera de servicio el cual puede ser evaluado a partir de la ecuación (3-9).

Finalmente, la ecuación (3-13) representa la contribución a la indisponibilidad asociada con una tarea de mantenimiento no programada, donde $f_{N}$ y $d_{N}$ representan la frecuencias y el tiempo fuera de servicio, respectivamente, para dicha actividad, siendo por ejemplo $f_{N}=\lambda$ para el caso concreto de una actividad de correctivo por fallo. La función G[AOT] representa la probabilidad de finalizar la actividad no planificada antes del máximo tiempo permitido fuera de servicio, dado por el AOT. Dicha probabilidad puede ser formulada como:

$\mathrm{G}[\mathrm{AOT}]=1-\mathrm{e}^{-\frac{\mathrm{AOT}}{\mathrm{d}_{\mathrm{N}}}}$

donde el $A O T$ varía dentro del intervalo $[0,+\infty[$.

Se observa que cuando un equipo no está sujeto a un AOT, entonces se considera que $A O T \rightarrow \infty$ y por tanto $G[A O T] \rightarrow 1$.

\subsection{Modelos de seguridad}

Es una práctica frecuente en las centrales nucleares considerar el riesgo como medida de seguridad de la planta. En este contexto, el riesgo puede definirse como la probabilidad de ocurrencia de un accidente por el daño que el mismo puede causar. A partir del Análisis Probabilista de Seguridad (APS) de las CC.NN. se puede cuantificar, entre otros, el modelo de seguridad. Así, 
es posible obtener las contribuciones asociadas al incremento del riesgo condicional, al riesgo simple y al riesgo anual.

El riesgo simple representa el riesgo esperado en el intervalo que un componente o equipo se encuentra fuera de servicio, el cual se puede formular como:

$\mathrm{r}=\Delta \mathrm{R} \cdot \mathrm{AOT}$

donde el término $\Delta R$ representa el incremento de riesgo condicional de una central nuclear cuando un sistema relativo a la seguridad tiene una desviación respecto a su punto normal de funcionamiento, con respecto del riesgo de la central en el caso base, en el que se supone que el sistema funciona según lo esperado (Samanta 1995) usando por lo general la información del APS y los conjuntos mínimos de corte que definen el riesgo considerado de la central.

La contribución al riesgo anual de la central, como consecuencia de las posibles indisponibilidades del sistema que cabe esperar en promedio a lo largo de un año natural, se puede aproximar mediante la siguiente expresión:

$\mathrm{R}=\Delta \mathrm{R} \cdot \mathrm{u}$

donde $u$ representa la indisponibilidad asociada a un sistema dado la cual se cuantifica a partir de las expresiones (3-11), (3-12) y (3-13).

\subsection{Modelos de coste}

Además, cada pareja de causa de fallo dominante y tarea de mantenimiento lleva asociado una contribución al coste total del ciclo de vida del equipo, el cual corresponde a una de las siguientes ecuaciones de acuerdo con las contribuciones a las indisponibilidad anteriores según el tipo de actividad, que se formula en términos de coste anual medio como sigue (Martorell 2002):

$$
\begin{aligned}
& c_{S}=8760 \cdot f_{S} \cdot c_{1 S} \\
& c_{N}=8760 \cdot f_{N} \cdot c_{1 N}
\end{aligned}
$$


$\mathrm{c}_{\mathrm{D}}=8760 \cdot \mathrm{f}_{\mathrm{N}} \cdot(1-\mathrm{G}[\mathrm{AOT}]) \cdot \mathrm{c}_{1 \mathrm{D}}$

$\mathrm{c}_{\mathrm{A}}=\mathrm{R} \cdot \mathrm{c}_{1 \mathrm{~A}}$

La ecuación (3-17) representa la contribución al coste anual medio como consecuencia de llevar a cabo una prueba planificada o programada sobre el periodo de un año. La ecuación (3-18) representa la contribución al coste anual medio como consecuencia de ejecutar una tarea de mantenimiento no planificada o programada sobre el periodo de un año. Además las variables $c_{1 S}$ y $c_{1 N}$ en las ecuaciones (3-17) y (3-18) respectivamente, representan el coste unitario como consecuencia de ejecutar una única tarea programada o no planificada respectivamente, pudiendo ser formulados usando la siguiente relación:

$\mathrm{c}_{1}=\mathrm{c}_{\mathrm{F}}+\mathrm{N}_{\mathrm{P}} \cdot \mathrm{c}_{\mathrm{HP}} \cdot \mathrm{T}_{\mathrm{P}}+\mathrm{N}_{\mathrm{E}} \cdot \mathrm{c}_{\mathrm{HE}} \cdot \mathrm{T}_{\mathrm{E}}$

donde $c_{F}$ representa el coste fijo por tarea, incluyendo por ejemplo los costes del material, siendo usualmente $T_{p}=d$ y $T_{E}=\max \left\{d_{\min } \geq 0, d\right\}$, representando el tiempo empleado por $N_{P}$ y $N_{E}$ hombres del personal propio y externo, respectivamente. Además, $C_{H E}$ representa el coste horario para el personal externo suponiendo que se trate de una cantidad fija, mientras que $C_{H P}$ es el coste horario el personal propio, el cual no es constante ya que depende de un número de factores, por lo que se propone la siguiente ecuación:

$c_{H P}=\frac{N_{P} \cdot S}{N_{e q} \cdot \sum_{\forall A \in P} f_{A} \cdot T_{P A}}$

donde la variable desconocida $S$ y $N_{e q}$ representan el salario anual y el número de componentes similares respectivamente asignados a cualquiera del personal propio $N_{P}$. La agregación se extiende sobre todas las tareas programadas $\mathrm{o}$ no, que afectan al equipo siendo ejecutada por el personal $N_{P}$.

La ecuación (3-19) representa la contribución al coste anual medio asociado con el número de paradas de planta y su correspondiente pérdida de producción que es estimada que ocurra en el horizonte de un año como consecuencia de actividades de mantenimiento no programadas que excedan el tiempo máximo disponible (AOT). Recordar que para un equipo de 
seguridad cuyo $A O T \rightarrow \infty$, dicho término se anula por $G[A O T] \rightarrow 1$ según la ecuación (3-14), mientras que para un equipo crítico para producción, dicho AOT $\rightarrow 0$, lo que supone parada inmediata de la planta, con su correspondiente coste puesto que $G[A O T] \rightarrow 0$. Además, $c_{1 D}$ representa el coste unitario debido a una parada de planta.

La última contribución se introduce para tener en cuenta el coste anual medio asociado con posibles accidentes. La ecuación (3-20) presenta un modelo comúnmente utilizado para tener en cuenta esta contribución (Yang 1999), donde $c_{1 A}$, es el coste por accidente y $R$ es la contribución al riesgo anual medio dado por la ecuación (3-16). Así, por ejemplo, cuando se usa un APS de nivel 1 para determinar las diferentes medidas del riesgo para la seguridad y para la salud, $R$ representa la frecuencia de daño al núcleo por reactor-año, y $c_{1 A}$ es el coste debido a un daño al núcleo, que se puede estimar por ejemplo como se propone en (Yang 1999).

En general a partir del enfoque anterior se suele trabaja con valores promediados en el curso de un año como periodo de referencia para obtener un valor medio de coste anual.

\subsection{Incorporación de la dependencia temporal}

Para estudiar en profundidad la influencia que sobre los atributos RAMS $+C$ pueden tener cambios en los requisitos de pruebas y mantenimiento se hace necesario la formulación de modelos probabilísticos dependientes del tiempo. Este aspecto es particularmente importante para estudiar el efecto de las estrategias, pruebas y mantenimientos.

Para formular el comportamiento de la indisponibilidad dependiente del tiempo, se va a utilizar el siguiente modelo (Cepin2002b):

- Indisponibilidad del componente en espera antes de la primera prueba:

$$
\mathrm{u}(\mathrm{t})=\rho+1-\mathrm{e}^{-\lambda^{*} \mathrm{t}} \quad 0<\mathrm{t}<\left(\mathrm{T}_{\mathrm{p}}-\mathrm{T}_{\mathrm{t}} \mathrm{f}_{0}\right)
$$


- Indisponibilidad del componente durante la primera prueba

$$
\mathrm{u}(\mathrm{t})=1 \quad\left(\mathrm{~T}_{\mathrm{p}}-\mathrm{T}_{\mathrm{t}} \mathrm{f}_{0}\right)<\mathrm{t}<\mathrm{T}_{\mathrm{p}}
$$

- Indisponibilidad del componente en espera entre pruebas:

$\mathrm{u}(\mathrm{t})=\rho+1-\mathrm{e}^{\left.-\lambda^{*}\left(\mathrm{t}-\mathrm{T}_{\mathrm{p}}\right) \operatorname{modT}_{\mathrm{i}}\right)} \quad 0<\left(\left(\mathrm{t}-\mathrm{T}_{\mathrm{p}}\right) \bmod _{\mathrm{i}}\right)<\left(\mathrm{T}_{\mathrm{i}}-\mathrm{T}_{\mathrm{t}} \mathrm{f}_{0}-\mathrm{T}_{\mathrm{r}}\right)$

- Indisponibilidad del componente debido a reparación:

$$
\begin{array}{r}
u(t)=\rho+1-e^{-\lambda^{*} T_{i}}+\left(e^{-\lambda^{*} T_{i}}-\rho\right)\left(\rho+1-e^{\left.-\lambda^{*}\left(\left(t-T_{p}\right) \bmod T_{i}\right)-T_{i}\right)}\right) \\
\left.T_{t} f_{0}-T_{r}\right)<\left(\left(t-T_{p}\right) \operatorname{modT}_{i}\right)<\left(T_{i}-T_{t} f_{0}\right)
\end{array}
$$

- Indisponibilidad del componente durante las siguientes pruebas:

$$
\mathrm{u}(\mathrm{t})=1 \quad\left(\mathrm{~T}_{\mathrm{i}}-\mathrm{T}_{\mathrm{t}} \mathrm{f}_{0}\right)<\left(\left(\mathrm{t}-\mathrm{T}_{\mathrm{p}}\right) \operatorname{modT}_{\mathrm{i}}\right)<\mathrm{T}_{\mathrm{i}}
$$

dónde:

$u(t) \quad$ indisponibilidad dependiente del tiempo,

$t$ tiempo,

$\rho \quad$ probabilidad de fallo a la demanda,

$\lambda^{*} \quad$ tasa de fallos en función del modelo de mantenimiento imperfecto (p.e. PAS o PAR),

$T_{p} \quad$ tiempo hasta la primera prueba,

$T_{t} \quad$ duración de la prueba 
$T_{r} \quad$ tiempo medio de reparación,

$f_{0} \quad$ factor que especifica la porción de la duración de la prueba durante la cual el componentes están indisponible, y

mod función que devuelve el resto del cociente entre dos factores

En resumen, el modelo de indisponibilidad dependiente del tiempo para cada componente considera principalmente tres posibles estados en que cada componente se puede encontrar a lo largo de su vida operacional.

Primero, el componente se encuentra normalmente en espera; es por ello que la probabilidad de que un componente falle mientras está en espera aumenta con el paso del tiempo desde la última prueba, como una función de su tasa de fallos en espera, (3-23) y (3-25),

Esta indisponibilidad es restaurada a cero, a excepción de la contribución por demanda, después de la ejecución de la prueba. Cada componente se asocia con un tiempo de inicio de la primera prueba (relacionada en general con la estrategia de pruebas) y un intervalo entre pruebas.

Segundo, cada prueba introduce una contribución a la inoperabilidad de componente que depende directamente de la duración de la prueba e inversamente proporcional al intervalo entre pruebas, (3-24) y (3-27).

Tercero, se asume que hay reparaciones una vez que se ha detectado el fallo del componente después de una prueba, lo que introduce una contribución a la indisponibilidad que depende directamente del tiempo medio de reparación y de la probabilidad de detectar que el componente se encuentra fallado, este último dependiente del intervalo entre pruebas, (3-26).

En el modelo anterior de indisponibilidad del componente la tasa de fallos del componente es espera, $\lambda^{*}$, se va a formular utilizando los modelos de apartado 3.1 para tener en cuenta el efecto del mantenimiento.

Seguidamente, el modelo de indisponibilidad con dependencia temporal para un sistema, se deriva usando el árbol de fallos y evaluando la indisponibilidad de cada componente en el tiempo según el modelo de componentes presentado anteriormente. 
A partir de estas expresiones se pueden derivar para un periodo de tiempo determinado la evolución temporal de la indisponibilidad, su valor medio $\left(U_{m}\right)$ y máximo $\left(U_{t m}\right)$, siendo estos dos últimos los que se toman como referencia en la toma de decisiones.

Adicionalmente los modelos de coste, se derivan agrupando las contribuciones al coste de las pruebas y mantenimientos sobre todos los componentes del sistema. Estos costes normalmente se derivan en base a un año como periodo de referencia para obtener un valor medio de coste anual.

\subsection{Incorporación de la incertidumbre.}

Los modelos probabilistas utilizados para simular el impacto que las actividades de pruebas y mantenimiento tienen sobre los atributos RAMS $+C$ presentan incertidumbre, la cual puede ser categorizada como aleatoria o epistémica (Reinert 2006). La incertidumbre aleatoria refleja nuestra incapacidad para predecir sucesos aleatorios observables, mientras que la incertidumbre epistémica representa la confianza en el modelo y en los valores numéricos de sus parámetros. En muchos casos, hay un conocimiento limitado sobre el mejor modelo para representar un problema, y esto resulta en que para una modelo particular de mantenimiento imperfecto, hay múltiples modelos compitiendo que producen diferentes aproximaciones al mismo problema, p.e. los modelos PAS y PAR descritos en el apartado 3.1. Por otro lado, la incertidumbre en los parámetros está relacionada con el valor asociado a los parámetros en un determinado modelo, p.e. la efectividad del mantenimiento en los modelos en los modelos anteriores, así pues, incluso con un modelo conocido, los valores de los parámetros pueden ser desconocidos y por lo tanto el resultado mantener cierta incertidumbre.

Existe un tercer caso que sería la incertidumbre de completitud, incertidumbre asociada al hecho de no disponer de suficiente información y/o nivel de detalle que permita modelizar adecuadamente el comportamiento de los diferentes sistemas.

En esta tesis sólo se van a considerar las incertidumbres denominas de parámetros y de modelo, sin entrar en las de completitud. Se trata de estudiar el efecto que dichas incertidumbres tienen sobre el proceso de toma de decisiones y en los resultados alcanzados. 


\subsubsection{Incertidumbre en el modelo de fiabilidad y mantenibilidad}

La fiabilidad inherente de los componentes es a menudo separada entre la contribución de la tasa de fallos en espera dependiente del tiempo, $\lambda^{*} \cdot T / 2$ (donde $T$ representa un tiempo entre pruebas dado), los cuales son asociados con causas que pueden ocurrir mientras el componente se encuentra en espera entre pruebas, más una contribución cíclica por demanda, $\rho$, la cual se asocia con los fallos que son causados al iniciar la demanda de funcionamiento o cíclicamente. Tomando como referencia la contribución a la indisponibilidad de un componente sometido a pruebas debida a su no fiabilidad, esta viene dada según el modelo presentado en el apartado 3.4 como:

$\mathrm{u}_{\mathrm{r}}(\mathrm{x})=\rho+\frac{1}{2} \lambda^{*} \mathrm{~T}$

siendo

$x \quad$ vector de variables de decisión que representa los parámetros a optimizar

$\rho \quad$ probabilidad de fallos por demanda o cíclica

$\lambda^{*} \quad$ tasa de fallos en espera considerando los modelos PAS o PAR de mantenimiento imperfecto, $y$

$T \quad$ intervalo entre prueba (STI)

La tasa de fallo $\lambda^{*}$ sigue las siguientes relaciones promediadas obtenidas a partir de las expresiones del apartado 3.1 dependiendo del modelo considerado.

$\lambda^{*}=\lambda_{0}+\frac{1}{2} \alpha \cdot M \cdot \frac{(2-\varepsilon)}{\varepsilon}$, modelo PAS,

$\lambda^{*}=\lambda_{0}+\frac{1}{2} \alpha \cdot \mathrm{M} \cdot\left[1+(1-\varepsilon) \cdot\left(\frac{\mathrm{RP}}{\mathrm{M}}-1\right)\right]$, modelo PAR. 
En ambos casos se adopta un modelo de envejecimiento lineal, donde $\lambda_{0}$ representa la tasa de fallos en espera residual, $\alpha$ representa el factor de envejecimiento lineal, $\varepsilon$, representa la efectividad del mantenimiento dentro del rango ]0,1], $\mathrm{M}$ el periodo para llevar a cabo un mantenimiento preventivo por tiempo y $R P$ el periodo tras el cual se lleva a cabo el reemplazamiento del componente.

Por tanto la adopción del modelo de mantenimiento más apropiado, PAS o PAR, introduce incertidumbre que afecta al resultado final del problema de optimización. Para tener en cuenta este tipo de incertidumbre, en la presente tesis, la selección de modelo de mantenimiento imperfecto se ha caracterizado mediante una distribución dicotómica de parámetro $p$, donde $p$ y (1-p) son las probabilidades de adoptar un modelo de mantenimiento imperfecto PAS o PAR, respectivamente, según la siguiente expresión (3-31).

$\lambda^{*}=p \cdot \lambda_{\mathrm{PAS}}^{*}+(1-\mathrm{p}) \cdot \lambda_{\mathrm{PAR}}^{*}$

En (Samanta 1995) se enfatiza la importancia de separar de forma apropiada la contribución dependiente del tiempo (tasa de fallos) y la referente de la demanda, al modelar la fiabilidad inherente del componente, por las consecuencias en la evaluación de la efectividad de las pruebas en lo que se refiere al riesgo limitado por la prueba asociado con un fallo ocurrido entre pruebas. El riesgo limitado por la prueba, significa en este contexto la indisponibilidad del sistema limitada por las pruebas asociadas con los fallos del componente entre pruebas. Así, la prueba de los componentes del sistema limita la indisponibilidad no detectada asociada con fallos entre pruebas o demandas reales para operar.

El riesgo limitado por la prueba solo concierne a la contribución afectada por la tasa de fallos en espera. Entonces, la descomposición de la probabilidad de fallo del componente en una contribución temporal y otra cíclica por demanda proporciona unos riesgos limitados por las pruebas menores porque solo una parte de los fallos es tratada como fallo en espera de naturaleza dependiente del tiempo. Por tanto, con esta separación se puede subestimar el riesgo limitado (subestima la efectividad de la prueba). Por el contrario, tratar toda la probabilidad de fallo asociado a la tasa de fallos en espera cuando no es el caso, sobrestima el riesgo limitado por la prueba (sobreestima la efectividad de la prueba).

A menudo, solo la probabilidad de fallo, esto es, la no fiabilidad del componente $u_{r}(x)$, es conocida por datos de campo. En esos casos, el efecto de un cambio en $T$ no puede ser evaluado a menos que el intervalo actual de 
pruebas del componente, $T$, y el periodo actual de mantenimiento, $M$, sean usados para convertir la no fiabilidad en términos de contribuciones $\rho$ y $\lambda^{*} \cdot T / 2$. Entonces, la separación entre las contribuciones temporales y cíclicas por demanda es necesaria pero desconocida.

Por tanto, la incertidumbre al separar la probabilidad de fallo del componente necesita ser tenida en cuenta. Esta incertidumbre epistémica es tenida en cuenta en este estudio mediante la reformulación de la probabilidad de fallo por demanda y la tasa de fallos en espera de cada componente utilizando las siguientes relaciones:

$$
\rho=\theta \cdot \rho_{\mathrm{m}}
$$

$\lambda_{0}=(1-\theta) \cdot \lambda_{0 m}$

donde $\theta$ es una varia le aleatoria que sigue una distribución uniforme en el rango $[0,1]$

$\theta=$ Uniform $[0,1]$

como se puede ver en (Helton 2004), de acuerdo con el principio de razón insuficiente (también llamado principio de indiferencia), se usa una distribución uniforme para caracterizar la incertidumbre epistémica en ausencia de información para distinguir la credibilidad de alternativas, y es además apropiada si uno realmente cree que cada uno de los valores del intervalo de distribución es igualmente apropiado.

De estas ecuaciones los valores de $\rho_{m}$ y $\lambda_{o m}$ son aquellos que corresponderían con la hipotética situación donde solo uno de ellos (fallo por demanda o tasa de fallos) es el contribuyente neto a la indisponibilidad $u_{r}(x)$ actual.

\subsubsection{Incertidumbre de parámetros}

Dentro de las posibles fuentes de incertidumbre, se encuentran aquellas relativas a los parámetros de los modelos utilizados. Principalmente se considera el efecto de la incertidumbre en la fiabilidad de los equipos, en particular, la incertidumbre de los parámetros de fiabilidad que representan los fallos en espera y los fallos en demanda de los equipos $(\rho, \lambda, \ldots)$, así como 
otros que representan la efectividad del mantenimiento $(\varepsilon)$.

No solo se presenta incertidumbres en estos tipos de parámetros, sino que también puede existir incertidumbres respecto a las variables de decisión, por ejemplo, no considerar a los intervalos entre pruebas $(T)$, mantenimientos $(M)$, etc. como valores constantes sino variables aleatorias dentro de unos intervalos determinados.

Las incertidumbres de parámetro se van a representar en esta tesis utilizando métodos probabilistas. De tal manera que los parámetros afectados por incertidumbres se formulan mediante funciones de densidad de probabilidad. Se procede de manera análoga para representar la incertidumbre en las variables de decisión. 



\section{Algoritmos evolutivos en optimización}

\subsection{Planteamiento del problema}

El problema de optimización multiobjetivo en base a criterios RAMS $+C$ puede formularse como un problema de toma de decisión multicriterio (TDMC). La Figura 4-1 muestra un esquema con las estrategias más comunes utilizadas para formular y resolver este tipo de problemas. Básicamente, la resolución del problema multiobjetivo puede llevarse a cabo mediante la resolución de distintos problemas de optimización simple objetivo que nos conducen a una única solución, o bien, abordando el problema multiobjetivo que nos dará un conjunto de soluciones óptimas al problema.

En general, un Problema de Optimización Multiobjetivo (POMO) incluye un conjunto de parámetros (variables de decisión), una función multiobjetivo y un conjunto de restricciones. Las funciones objetivo y las restricciones se formulan dependiendo explícitamente de las variables de decisión. Adaptando la definición formal del POMO para el campo de la optimización basada en múltiples criterios RAMS $+C$, éste puede plantearse de forma general como un problema de minimización de una función multiobjetivo de la forma (Zitzler 1999): 


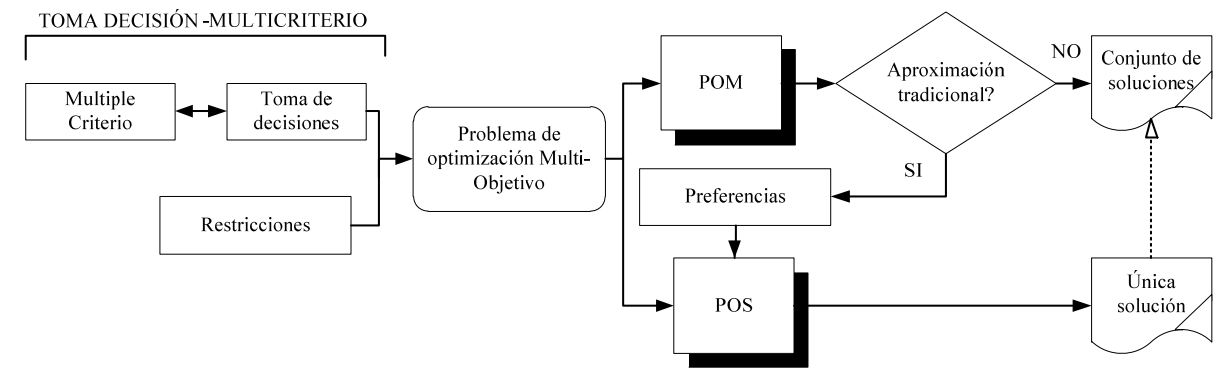

Figura 4-1: Estrategias de resolución de Problemas de Optimización MultiObjetivo.

$y(x)=f(x)=\left(f_{1}(x), f_{2}(x), \ldots, f_{n}(x)\right)$

sujeto al vector de restricciones

$\mathrm{g}(\mathrm{x})=\left(\mathrm{g}_{1}(\mathrm{x}), \mathrm{g}_{2}(\mathrm{x}), \ldots, \mathrm{g}_{\mathrm{n}}(\mathrm{x})\right) \leq 0$

donde

$\mathrm{x}=\left(\mathrm{x}_{1}, \mathrm{x}_{2}, \ldots, \mathrm{x}_{\mathrm{n}}\right) \in \mathrm{X}$

$\mathrm{y}=\left(\mathrm{y}_{1}, \mathrm{y}_{2}, \ldots, \mathrm{y}_{\mathrm{n}}\right) \in \mathrm{Y}$

siendo $x$ es el vector de decisión, $y$ el vector objetivo, $X$ el espacio de decisión e $Y$ el espacio objetivo. Esto es, $Y=f(X)$.

La mayoría de problemas reales plantean la búsqueda de la mejor solución bajo ciertas condiciones impuestas sobre los parámetros o variables de decisión. Las restricciones impuestas en el vector restricción $g(x)$ limitan la región de soluciones posibles, y por tanto, se denominan restricciones de dominio. Dichas restricciones pueden ser tanto explícitas o implícitas (Carlson 1995a) y (Carlson 1995b).

Así, supongamos que las restricciones impuestas por $g(x)$ fijan el conjunto de vectores de decisión posibles, $X_{p}$, tal que

$X_{p}=\{x \in X / g(x) \leq 0\}$ 
Este tipo de restricciones se denominan explícitas por cuanto se establecen directamente sobre las variables de decisión $x$. La imagen de $X_{p}$ corresponde a la región de soluciones posibles en el espacio objetivo, que se representa por el conjunto $Y_{p}$.

Alternativamente, es posible establecer restricciones sobre el valor alcanzado por las soluciones en el espacio objetivo, es decir, actuando sobre el conjunto $Y_{p}$. Este tipo de restricciones se denominan implícitas por cuando a través de fijar el espacio de soluciones se fija de forma indirecta el espacio de decisión posible $X_{p}$.

Las primeras incluyen, por ejemplo, los valores que pueden adoptar los parámetros que van a actuar como variables de decisión en el problema de optimización. En este caso es fácil comprobar que se cumplen las restricciones, puesto que al elegir el valor de una variable, si no cumple con la restricción, ya se sabe que no es un resultado factible en el proceso de optimización. Por otro lado, una restricción implícita es aquélla que se impone sobre las funciones objetivo $\mathrm{y}$, por tanto, requiere una simulación para comprobar la violación o no de la misma. Por ejemplo, es necesario evaluar el coste o el riesgo asociado con una serie de valores de las variables de decisión para asegurar que ninguno de ellos está fuera de los límites fijados.

La optimización con restricciones y la optimización multiobjetivo pueden verse como dos aspectos del mismo problema. Así, las restricciones pueden considerarse como objetivos prioritarios (fuertes) que deben alcanzarse antes que el resto de objetivos del proceso de optimización (débiles), representados estos últimos en el vector objetivo $f(x)$. De hecho, en la búsqueda de soluciones al problema de toma de decisión multicriterio, los criterios pueden integrarse en el problema de optimización a través del vector objetivo, del vector restricción o de ambos según el juicio del analista. En muchos casos, el que se verifiquen las restricciones ya es un problema en sí mismo. Cuando no se pueden verificar todas las restricciones simultáneamente se dice que el problema no admite solución. En este caso, resulta necesario introducir una mayor flexibilidad, bien reduciendo el número de restricciones o bien relajándolas.

$\mathrm{Si}$, por ejemplo se pretende estudiar el caso particular en el que se pretende maximizar la disponibilidad (Availability) de un sistema de seguridad, o minimizar su indisponibilidad (Unavailability), minimizando los costes (C) asociados al mantenimiento $(A[U]+C)$, el problema puede formularse como sigue: 
$y=f(x)=(U(x), C(x))$

$\mathrm{g}(\mathrm{x})=\left(\mathrm{U}(\mathrm{x}) \leq \mathrm{U}_{\mathrm{i}}, \mathrm{C}(\mathrm{x}) \leq \mathrm{C}_{\mathrm{i}}\right)$

donde

$x \in X=\{$ rango de valores permitidos para la variable de decisión $\}$

$y \in Y=\{$ rango de valores permitidos para el vector objetivo $\}$

$U_{i}=U\left(x_{i}\right)=$ indisponibilidad asociada con los valores iniciales (por defecto) para el vector decisión.

$C_{i}=C\left(x_{i}\right)=$ costes asociados con los valores iniciales (por defecto) para el vector decisión.

Se pueden encontrarse un gran número de publicaciones dedicadas a resolver este tipo de problemas utilizando técnicas de optimización (Zitzler 1999) que incorporan múltiples objetivos y múltiples restricciones. La Figura 4-1 muestra los dos grandes grupos de alternativas utilizadas comúnmente para resolver el problema de optimización multiobjetivo (POMO). En general, un POMO admite múltiples soluciones. De esta manera el que toma la decisión debe finalmente emitir un juicio de valor (preferencia) que permita priorizar entre un conjunto de soluciones posibles para llegar a una solución final única. La manera en la que se impone dicha preferencia se puede clasificar en tres grandes líneas (Zitzler 1999):

a. Toma de decisiones antes de la búsqueda (conocida como aproximación tradicional que transforma el POMO en un POSO (Problema de Optimización Simple Objetivo) mediante la imposición a priori de la preferencia. Ver la Figura 4-1, donde los objetivos del POMO se agregan para formar un único objetivo del POSO que incluye de manera implícita la preferencia,

b. Búsqueda de soluciones antes de la toma de decisión final, donde se resuelve directamente el POMO sin establecer ninguna preferencia a priori y llegando a un conjunto de soluciones óptimas a partir de las cuales se debe llevar a cabo la elección final utilizando el criterio de preferencia, y 
c. Toma de decisiones durante la búsqueda, donde se articula la preferencia en un procedimiento de optimización interactivo que guía la búsqueda desde el espacio de soluciones actual hacia el espacio fijado por la nueva preferencia articulada a partir del análisis de las soluciones obtenidas hasta este momento.

Lo habitual ha sido utilizar una de las dos alternativas extremas, a) o b). Así, en el caso de la primera, el POMO se transforma en uno o varios problemas de optimización simple objetivo (POSO), mientras que en el segundo se resuelve de manera directa el $\mathrm{POMO}$. La alternativa $\mathrm{POSO}$ fue la primera en ser utilizada, mientras que la alternativa $\mathrm{POMO}$ es más reciente.

\subsubsection{Problema de Optimización Simple Objetivo. Solución única}

El Problema de Optimización Simple Objetivo (POSO) corresponde a un caso particular del POMO, que se da cuando se considera un solo criterio, o bien, cuando se consideran varios criterios aunque tan sólo uno de ellos, o una combinación de ellos, actúa como objetivo, basado en una decisión tomada a priori, mientras que el resto se introducen como restricciones al problema (Zitzler 1999). La mayor parte de métodos clásicos de optimización desarrollados en el pasado se han centrado en la resolución de problemas POSO, tales como métodos del gradiente, búsqueda directa, métodos heurísticos (Monte Carlo, Algoritmos Evolutivos, etc.). Por ejemplo, en el caso particular $\mathrm{A}[\mathrm{U}]+\mathrm{C}$ comentado con anterioridad es posible encontrar soluciones a problemas simple objetivo, de manera que una solución minimice una función objetivo (p.e. $U(\boldsymbol{x})$ ) y otra solución minimice una función objetivo distinta (p.e. $C(\boldsymbol{x})$ ), utilizando métodos clásicos o avanzados. Sin embargo, ambas soluciones normalmente no son la misma, y, además, no se puede mejorar un objetivo sin empeorar el otro, puesto que en este caso los dos criterios considerados son antagónicos, es decir, una mejora en la fiabilidad exige un aumento del coste

\subsubsection{Problema de Optimización Multiobjetivo. Múltiples soluciones. Conjunto de soluciones óptimas no dominadas de Pareto $y$ frontera de Pareto.}

En este caso la búsqueda de la solución se lleva a cabo sin una toma de decisiones a priori, lo cual proporcionará un conjunto de diferentes soluciones al problema, por lo que se hace necesaria una decisión final a posteriori para elegir la solución individual más adecuada. 
En general, la solución a un problema POMO siempre se sitúa en lo que se denomina como conjunto (no dominado) óptimo de Pareto (Zitzler 1999), el cual queda conformado por un número de soluciones posibles no dominadas.

Se dice que un vector decisión solución, $x$, es no dominado si no existe ningún otro vector solución que sea mejor que él simultáneamente con respecto a todas las funciones objetivo. A cada una de las soluciones no dominadas del problema POMO se la denomina solución óptima de Pareto, $x^{*}$, y en su totalidad forman el conjunto de soluciones óptimas no-dominadas de Pareto. La imagen de este conjunto en el espacio objetivo constituye lo que se denomina frontera de óptimos de Pareto, $y^{*}=f\left(x^{*}\right)$.

En teoría, todas las soluciones del conjunto óptimo de Pareto resultan de interés y por tanto deberían ser conocidas. Sin embargo, el conjunto óptimo de Pareto no ayuda a seleccionar una solución, por lo que es, en definitiva, el analista quien en último caso debe emitir un juicio de valor acerca de las diferentes alternativas para tomar una decisión, ya que en general, el POMO no tiene una única solución que pueda optimizar simultáneamente todos los objetivos (Goldberg 1989). No obstante, el conjunto de soluciones óptimas de Pareto ayuda al analista a balancear las distintas soluciones bajo la perspectiva de su situación respecto al valor alcanzado en las funciones objetivo.

Así pues, el analista encargado de la toma de decisiones, bien a priori o a posteriori del proceso de optimización, debe articular una función de preferencia de las posibles soluciones al problema de optimización multiobjetivo. El hecho de establecer la función de preferencia a priori o a posteriori condiciona el método de optimización utilizado en la búsqueda de soluciones.

\subsection{Toma de decisiones en base a criterios RAMS+C.}

En general, se puede decir que la optimización de la fiabilidad y disponibilidad de los sistemas se basa tradicionalmente, en la cuantificación de los efectos que la elección del diseño y de las actividades de pruebas y mantenimiento tienen sobre los atributos RAM. Para ello, se usa un modelo cuantitativo que determine cómo las elecciones de diseño y mantenimiento afectan a los atributos RAM del sistema y a los costes asociados. Así, el problema de optimización del diseño y mantenimiento debe ser tratado como un Problema de Decisión Multicriterio (TDMC) donde los atributos RAMS $+C$ actúan como criterios de decisión, opuestos entre sí, siendo entonces funciones objetivo y los parámetros relevantes de diseño y mantenimiento (por ejemplo, 
configuraciones redundantes, tasas de fallo de los componentes, periodicidad de los mantenimientos, frecuencias de pruebas, etc.) actúan como variables de decisión.

En particular, para industrias con un cierto nivel de riesgo, tales como las centrales nucleares, la toma de decisiones para la optimización del diseño y mantenimiento de los sistemas de seguridad, también tiene en cuenta los atributos de riesgo, los cuales integran los efectos que las elecciones del diseño y el mantenimiento ejercen sobre el sistema como un todo mediante la inclusión de la probabilidad de ocurrencia de los sucesos potencialmente peligrosos y las consecuencias que se esperan.

Por todo ello, la toma de decisiones sobre pruebas y mantenimiento para centrales nucleares implica la consideración simultánea de criterios RAM+Safety (Seguridad). Por ejemplo, la optimización de las actividades de pruebas y mantenimientos de sistemas relacionados con la seguridad apunta al incremento de los atributos RAM los cuales, derivan en una mejora del nivel de seguridad de la planta. Esto, sin embargo, se obtiene a expensas de un incremento de inversión en los recursos ( $p$. e. costes de material y realización de la prueba, recursos humanos, etc.). Por lo tanto, la tarea de la toma de decisiones multicriterio conduce a encontrar las configuraciones apropiadas de diseño de la fiabilidad, estrategias de pruebas y mantenimientos que optimice el balance entre los atributos RAMS y Costes. Para este objetivo, para cada solución potencial, $\boldsymbol{x}$, se evalúan los atributos de fiabilidad, disponibilidad, mantenibilidad, riesgo/seguridad y costes, de manera que se obtenga un resultado numérico de cada uno.

Por lo tanto, el primer paso para facilitar la toma de decisiones es formular matemáticamente la relación entre los criterios y las variables de decisión mediante modelos apropiados, con el propósito de simular el efecto que tiene sobre cada uno de los criterios de decisión el valor de las variables de decisión. En particular, los modelos utilizados para considerar los criterios RAMS $+C$ corresponden a:

- $\mathrm{R}(\mathbf{x})=$ Fiabilidad del equipo

- $\mathrm{A}(\mathbf{x})=$ Disponibilidad del equipo, que normalmente se evalúa en términos de indisponibilidad $\mathrm{U}(\mathbf{x})$ como $1-\mathrm{U}(\mathbf{x})$, que se representa de aquí en adelante como $\mathrm{A}[\mathrm{U}]$.

- $\mathrm{M}(\mathbf{x})=$ Mantenibilidad del equipo, la cual se evalúa como la indisponibilidad del equipo como consecuencia de la realización de las pruebas y el mantenimiento programado y no programado.

- $\mathbf{S}(\mathbf{x})=$ Nivel de Seguridad, la cual se evalúa normalmente en 
términos de riesgo (p.e. Análisis Probabilista del Riesgo de Centrales Nucleares), donde la variable Riesgo(x), contempla la frecuencia de un accidente y el daño para la salud y seguridad de la población, para el medio ambiente o para la propiedad según el tipo de riesgo estimado respectivamente riesgo para seguridad, riesgo medioambiental y riesgo económico.

- $\mathrm{C}(\mathbf{x})=$ Coste necesario para garantizar un nivel de RAMS dado por las variables anteriores.

Gran parte de las aplicaciones simplifican el problema de toma de decisiones considerando únicamente un subconjunto de los criterios RAMS $+\mathrm{C}$ anteriores (Martorell 2002), enfrentándose por ejemplo a problemas de mejora en base a criterios $A[U]+C$, donde únicamente se requiere de modelos detallados para representar la indisponibilidad del equipo o sistema, $U(x)$, y los costes asociados, $C(x)$.

Desde un punto de vista general, entre otros, el vector de variables de decisión $x$ incluye los parámetros relacionados con la fiabilidad inherente al equipo (p. e. probabilidad de fallo por demanda, tasa de fallos, etc.), configuración lógica de los sistemas (p.e. número de trenes redundantes, etc.), los cuales definen la asignación de la fiabilidad del sistema, y aquellos relevantes a las actividades de pruebas y mantenimiento (intervalos entre pruebas, periodicidad de los mantenimientos, periodos de renovación, efectividad de los mantenimientos, tiempos medios de reparación, tiempo permitido fuera de servicio, etc.), los cuales gobiernan las características de disponibilidad y mantenibilidad del sistema.

La elección de unos u otros parámetros para formar parte del vector de decisión depende en cada caso de hacia donde se oriente la mejora. Así, por ejemplo, ciertas aplicaciones se centran en los parámetros relacionados con las pruebas y el mantenimiento de los equipos de seguridad del sistema $(T$, $M, L, \varepsilon, \mu$, D, etc.) (Vaurio 1995), (Muñoz 1997a), (Martorell 2002), (Harunuzzaman 1996), (Busacca 2001), (Cepin 2002), (Muñoz 1997b), (Marseguerra 2000), (Lapa 2003), (Tsai 2001), (Yang 2000) y (Charles 2003), con el objetivo de optimizar la disponibilidad de dichos sistemas y los costes asociados, por lo que sólo se consideran los criterios de $A(U)+C$, mientras que otras tienen interés en los parámetros de diseño relativos a fiabilidad de equipos $(\mu, \lambda$, etc.), redundancias, etc., los cuales se encuentran más relacionados con el área de fiabilidad (Paiton 1995) y (Cantoni 2000), o bien con todos los ámbitos simultáneamente (Levitin 1999).

La aproximación más usual para llevar a cabo la búsqueda de soluciones realistas en este contexto de toma de decisiones multicriterio, tal y como ya 
se ha adelantado en la sección anterior, cae dentro del campo del Problema de Optimización Multiobjetivo (POMO). Son muchas y variadas las experiencias de aplicación de técnicas de optimización POMO para lograr soluciones a diversos problemas de ingeniería donde existen múltiples funciones objetivo y múltiples restricciones.

\subsection{Optimización mediante computación evolutiva: Algoritmos Genéticos.}

En la literatura pueden encontrarse distintos métodos de búsqueda de soluciones a problemas de optimización multiobjetivo. Los métodos heurísticos tienen un gran protagonismo, en especial los Algoritmos Evolutivos (AA.EE.) por sus características y comportamiento al resolver problemas POMO. Los Algoritmos Genéticos (AA.GG.) son un tipo concreto de AA.EE con una serie de características interesantes como son la de mantener la población de soluciones y al mismo tiempo buscar soluciones no dominadas. Esta característica favorece la búsqueda de un conjunto óptimo de Pareto al resolver un POMO.

\subsubsection{Orígenes, fundamentos y primeras aplicaciones.}

Los Algoritmos Genéticos son probablemente la técnica heurística de optimización más conocida dentro de los llamados Algoritmos Evolutivos, (Bäck 1996). Los AA.GG. son métodos adaptativos usados en los problemas de búsqueda y optimización, que trabajan imitando los principios de la selección natural y de la genética. Los AA.GG en su forma más común fueron desarrollados por John Holland en 1965 que resumió su trabajo en (Holland 1975). Este trabajo junto con otros de sus estudiantes (De Jong 1975) son los únicos documentos disponibles relativos a AA.GG. hasta finales de los setenta, los cuales sirvieron como punto de partida de casi todas las aplicaciones e implementaciones conocidas de los AA.GG.

En la literatura pueden encontrarse numerosos artículos (Beasley 1993a), (Beasley 1993b), (Fogel 1994) y (Marseguerra 2006), así como libros (Bäck 1996), (Goldberg 1989), (Davis 1991), (Fogel 1995) y (Michalewicz 1996), y tesis doctorales (Zitzler 1999), que presentan los fundamentos sobre cómo trabajan los AA.GG., su implementación y aplicaciones.

Debido a su simplicidad, flexibilidad, sencillez de operación, mínimo de requisitos y perspectiva global, los AA.GG. se han usado satisfactoriamente en una gran variedad de problemas en muchas áreas de la ingeniería y 
ciencias naturales, como se describe por ejemplo en (Beasley 1993b), (Fogel 1994) y (Herrera 1996).

A mediados de los 90 vieron la luz las primeras aplicaciones de los AA.GG en el campo de la optimización de la fiabilidad, mantenibilidad y disponibilidad de equipos (Paiton 1995), (Kumar 1995), (Muñoz 1997b) y (Levitin 1999), demostrando la capacidad de esta técnica de optimización en la resolución de este tipo de problemas.

\subsubsection{Evolución de los Algoritmos Genéticos.}

En un principio, los AAGG fueron desarrollados para abordar la optimización de problemas con una única función objetivo y sin restricciones. A medida que se avanzaba en el estudio de esta técnica heurística la complejidad de los problemas a resolver avanzó hacia la consideración de restricciones al problema y la optimización de varias funciones objetivo, es decir la consideración de un problema de optimización multiobjetivo.

Las primeras aproximaciones a la resolución de problemas de optimización multiobjetivo utilizando AA.GG. (Algoritmo Genético Multiobjetivo, AGMO) estaban basados en la transformación del problema original a un problema de optimización simple objetivo (Algoritmo Genético Simple Objetivo, AGSO). Existe un gran número de publicaciones en las que se utilizan AA.GG. para la optimización de requisitos de vigilancia y mantenimiento que se basan en la aproximación de un problema AGMO a uno AGSO. Sin embargo, tras un proceso de mejora de los AA.GG. se ha conseguido adaptar esta técnica de optimización para que resuelva directamente problemas de optimización multiobjetivo.

Como ya se ha comentado anteriormente, los AA.GG. se desarrollaron como técnicas de búsqueda sin restricciones, por lo que requieren de la asignación de medidas escalares de calidad, ajuste, o ponderación de las soluciones candidatas del problema de optimización de forma que la solución última cumpla con las restricciones al problema. Ha sido necesaria una mejora en los AA.GG. para que se obtengan buenos resultados en la optimización de problemas con restricciones, que son los más interesantes, ya que la mayoría de los problemas de optimización en ingeniería presentan a menudo una solución acotada por un determinado número de restricciones impuestas a las variables de decisión (Joines 1994), (Michalewicz 1995), (Carlson 1995a), (Carlson 1995b), (Martorell 1995) y (Leemis 1995).

Se han considerado muchos tipos de aproximaciones en el AGSO para el 
manejo de soluciones que no violen una o más restricciones, pero ningún método general ha sobresalido sobre el resto. Estas aproximaciones se pueden categorizar como sigue (Joines 1994), (Michalewicz 1995), (Carlson 1995a) y (Carlson 1995b):

1. Eliminar las soluciones no factibles.

2. Reparar las soluciones no factibles.

3. Usar operadores genéticos modificados.

4. Penalizar las soluciones no factibles.

La primera aproximación se utiliza con éxito en (Muñoz 1997b), (Levitin 1999) y (Goldberg 1989) aunque proporciona resultados pobres especialmente en los problemas con grandes restricciones. La reparación de soluciones no factibles y la utilización de operadores genéticos modificados son métodos más útiles para el manejo de restricciones explícitas ya que en el caso de restricciones implícitas estas técnicas requieren un tiempo computacional elevado. La última aproximación, es la técnica que más se utiliza para el manejo de restricciones implícitas, y además también permite el manejo de restricciones explícitas. Esta técnica transforma el problema restringido en un problema sin restricciones mediante la penalización de aquellas soluciones que no son factibles después de la evolución. En el campo de la optimización del diseño, pruebas y mantenimiento basado en $R A M S+C$ se utiliza principalmente la penalización para el manejo de las restricciones implícitas como es el caso de la presente tesis.

\subsubsection{Desarrollo de Algoritmos Genéticos Multiobjetivo}

El AGMO afronta el problema de optimización multiobjetivo de una forma directa, es decir considerando todas las funciones objetivo. El primer AGMO fue desarrollado por Schaffer (Schaffer 1985), que propuso el VEGA (Vector Evaluation Genetic Algorithm). Más adelante, Kursawe desarrolló el algoritmo ESMO (Kursawe 1991), el cual afrontaba el problema multiobjetivo de una forma no agregada. Estas primeras aproximaciones se conocen como aproximaciones No-Pareto.

Goldberg (Goldberg 1989) fue el primero en proponer la aproximación basada en fronteras de Pareto que es una de las metodologías más utilizadas por los AGMO. La aproximación mediante fronteras de Pareto consiste en asignar igual probabilidad de reproducción para todos los individuos no dominados de la población. Este concepto de dominancia de Pareto es bien conocido y se encuentra descrito con detalle en (Goldberg 1989), (Zitzler 1999) y 
(Marseguerra 2006).

Dentro de esta aproximación genética, existen tres grandes problemas que deben tenerse en cuenta cuando se pretende aplicar un algoritmo genético a un problema de optimización multiobjetivo:

1. Cómo medir el ajuste de una solución y cómo llevar a cabo la selección para guiar la búsqueda hacia el conjunto óptimo de Pareto. Esto se consigue considerando el concepto de dominancia de Pareto.

2. Cómo mantener la diversidad de la población para evitar una convergencia prematura y alcanzar un conjunto bien distribuido y extenso de soluciones no dominadas. Esto se consigue mediante una estimación de la densidad.

3. Como evitar la pérdida de los mejores individuos a lo largo del proceso de evolución. Esto se consigue a través del "elitismo".

Por lo que concierne al manejo de las restricciones, ya sean explícitas o implícitas, se tratan de forma similar al caso de los algoritmos genéticos simple objetivo, comprobando si en el curso del proceso de reproducción y reemplazamiento de la población, la solución candidata cumple con las restricciones. Puede adoptarse, por ejemplo, una aproximación mediante penalizaciones dinámicas, para degradar el ajuste de aquellos individuos que pueden violar alguna restricción de alguno de los objetivos. Finalmente, existen otros métodos basados en este caso en la restricción de la dominancia que aplicarían únicamente en el caso de problemas multiobjetivo.

Se han desarrollado un buen número aproximaciones de primera generación de AGMO basadas en la frontera de Pareto: MOGA (Multiobjective Genetic Algorithm) (Fonseca 1993), NSGA (Non-dominated Sorting Genetic Algorithm) (Srinivas 1994), y NPGA (Niched Pareto Genetic Algorithm) (Horn 1994). Estos algoritmos tienen propiedades comunes como que las soluciones se clasifican de acuerdo a su dominancia entre la población y se mantiene la diversidad utilizando estrategias de "colocación".

La segunda generación de Algoritmos Genéticos representa una evolución de la primera generación de aproximaciones de Pareto, en las cuales se implementa el uso del elitismo para mejorar la convergencia (en términos de fiabilidad del resultado y velocidad del algoritmo). EI SPEA (Strength Pareto Evolutionary Algorithm) (Zitzler 1999), SPEA2 (Zitzler 2001), NSGA-II (Deb 2002), y PAES (Pareto Archived Evolution Strategy) (Knowles 1999) son ejemplos de esta generación de AA.GG. 
Por lo que se refiere a la determinación de la bondad de la función objetivo, existen tres métodos típicos de clasificación basada en la dominancia:

1. Rango de dominancia: Basado en calcular cuántos individuos de la población dominan a un determinado individuo (Fonseca 1993).

2. Profundidad de dominancia: Que consiste en conocer a qué frontera de individuos no-dominados pertenece un individuo (Deb 2002).

3. Contador de dominancia: Basado en calcular a cuántos individuos de la población domina un cierto individuo (Zitzler 2001).

Para mantener la diversidad, segundo gran problema a tener en cuenta en la optimización, las técnicas utilizadas con más frecuencia en la optimización multiobjetivo se basan en la estimación de la densidad de la población. Un ejemplo de dicha estimación consiste en compartir el valor de la función objetivo entre los individuos cercanos de una misma población (sharing fitness) (Fonseca 1993), (Deb 2002). Otra técnica que puede utilizarse consiste en calcular la densidad en cualquier punto en el espacio de la solución como una función decreciente de la distancia al k-ésimo punto más cercano (Zitzler 2001).

Finalmente la implementación del concepto de elitismo, para evitar la pérdida de mejores individuos a lo largo del proceso de evolución, en el algoritmo genético multiobjetivo se consigue guardando la traza de los mejores individuos de cada generación (Deb 2002) y (Zitzler 2001).

En paralelo, se ha extendido el uso de métricas para el apoyo en la comparación de soluciones proporcionadas por diferentes ejecuciones de un algoritmo genético para resolver el mismo problema multiobjetivo. Se han introducido métricas de ejecución cuantitativas para determinar la eficiencia de los algoritmos genéticos multiobjetivos en lo concerniente tanto a precisión, como al grado de cobertura de la frontera de Pareto (Zitzler 1999), (Laumanns 2002) y (Zitzler 2003).

\subsection{Aproximaciones AGSO y AGMO.}

Con la finalidad de simplificar la explicación de las aproximaciones desarrolladas, en los apartados siguientes se describen, ambas aproximaciones AGSO y AGMO, para el caso en concreto en el que se trate 
el problema con dos criterios: Disponibilidad (indisponibilidad) y Coste.

\subsubsection{AGSO basado en la efectividad}

Para resolver el problema multiobjetivo mediante su transformación a un problema simple objetivo utilizando algoritmos genéticos (AGSO) se ha adoptado un Algoritmo Genético Estacionario (Steady-State Genetic Algorithm, SSGA) descrito en (Martorell 2000) usando una nueva función objetivo basada en el concepto de efectividad para resolver el problema de optimización considerando los criterios de indisponibilidad y coste $(U+C)$.

La aproximación basada en la efectividad para la búsqueda de soluciones al problema de optimización multiobjetivo combina tres aproximaciones clásicas: método de las importancias o pesos, de restricciones, y distancias. Este proceso se puede ver como una aproximación tradicional que transforma el problema de optimización multiobjetivo en un número determinado de problemas de optimización simple objetivos que se resuelven de forma secuencial, y que podría proporcionar un conjunto de soluciones cercanas o pertenecientes a la frontera óptima de Pareto.

La Figura 4-2 muestra un gráfico U-C que representa el espacio objetivo de las posibles soluciones al problema de optimización multiobjetivo, en particular al problema U+C, centrándose en la optimización simultánea de la funciones de indisponibilidad y coste bajo varias restricciones para un sistema de seguridad. Cada una de las posibles soluciones del problema, codificadas en el vector $x$, tiene asociada unos vectores objetivo y restricción de la forma dada por las ecuaciones (4-4) y (4-5) respectivamente.

En el espacio objetivo mostrado por la Figura 4-2, el punto $\left\{U_{i}, C_{i}\right\}$ representa el valor alcanzado por cada función objetivo con los valores iniciales asignados a las variables de decisión. Por ejemplo, para el problema $A[U]+C$, los valores iniciales para la indisponibilidad del sistema y costes asociados se obtienen de la cuantificación de estos criterios utilizando los modelos desarrollados en el capítulo 3, y los valores para los intervalos entre pruebas y mantenimiento reales de la planta.

También en la Figura 4-2, el punto $\left\{U_{m}, C_{m}\right\}$ representa un punto hipotético, normalmente imposible de alcanzar, donde los valores de ambas funciones objetivo alcanzan, simultáneamente, el mínimo valor posible. 


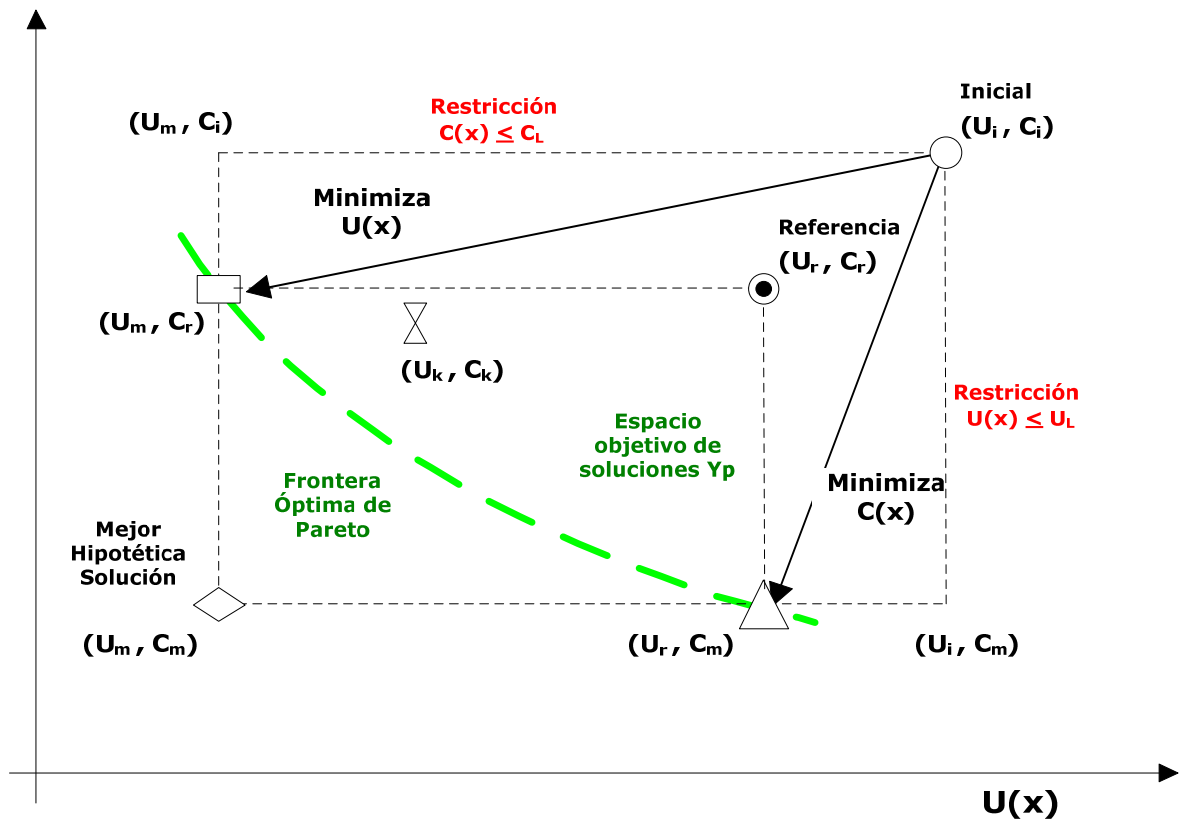

Figura 4-2: Espacio objetivo de las posibles soluciones para el AGSO basado en la efectividad.

El valor $C_{m}$ se obtiene resolviendo un problema de optimización simpleobjetivo, donde la función objetivo considerada es la función coste, $y=C(x)$, mientras que la función indisponibilidad se trata como una restricción de la forma $U(x) \leq U_{i}$, la cual se añade al conjunto de restricciones del problema original. De igual forma, el valor $U_{m}$ se obtiene al minimizar la función indisponibilidad, considerada como función objetivo $y=U(x)$, y tratando el coste como una restricción de la forma $C(x) \leq C_{i}$, la cual se añade al conjunto de restricciones del problema original.

Los puntos obtenidos después de resolver ambos problemas de optimización definidos anteriormente definen dos vértices opuestos de un rectángulo, ver Figura 4-2, representado por $\left\{U_{m}, C_{i}\right\}$ y $\left\{U_{i}, C_{m}\right\}$ que determinan la región teórica de soluciones posibles para el problema de optimización multiobjetivo.

El concepto de efectividad se introduce para tener en cuenta aquellas situaciones en las que los valores iniciales de los parámetros de decisión se encuentran muy alejados de los valores óptimos, por lo que se hace 
interesante redefinir los valores iniciales por otros más próximos a la mejor solución obtenida de un problema simple objetivo previo, denominado punto de referencia. Esta situación podría entenderse, por ejemplo, como que las actuales actividades de pruebas y mantenimiento implementadas en la planta se encuentran bastante alejadas de ser óptimas, y la búsqueda de una buena solución debería empezar desde un punto diferente con el objetivo de guiar la búsqueda de una manera más rápida hacia las mejores soluciones. Es entonces cuando se sugiere el uso de un punto de referencia para definir la efectividad para una solución $k$ dada como sigue:

$$
\begin{aligned}
& \mathrm{e}_{\mathrm{u}}\left(\mathrm{x}_{\mathrm{k}}\right)=\frac{\mathrm{U}_{\mathrm{r}}-\mathrm{U}_{\mathrm{k}}}{\mathrm{U}_{\mathrm{r}}-\mathrm{U}_{\mathrm{m}}} \\
& \mathrm{e}_{\mathrm{c}}\left(\mathrm{x}_{\mathrm{k}}\right)=\frac{\mathrm{C}_{\mathrm{r}}-\mathrm{C}_{\mathrm{k}}}{\mathrm{C}_{\mathrm{r}}-\mathrm{C}_{\mathrm{m}}}
\end{aligned}
$$

donde $U_{r}$ es la indisponibilidad asociada con el mínimo coste encontrado en el problema de optimización que considera como función objetivo el coste considerando la indisponibilidad como restricción y $C_{r}$ es el coste asociado con la mínima indisponibilidad encontrada en el problema de optimización que considera como función objetivo la indisponibilidad y trata el coste como restricción.

Haciendo uso de la definición de efectividad propuesta, se construye la función objetivo para el AGSO como sigue:

$y=f(x)=1-\left\{\omega_{u} e_{u}(x)+\omega_{c} e_{c}(x)\right\}$

donde $\omega_{u}$ y $\omega_{c}$ son los dos coeficientes de ponderación que introducen flexibilidad en la búsqueda de la mejor solución que minimice la ecuación (4-8), lo cual en otras palabras representa la importancia que se le asigna a las funciones indisponibilidad y coste, respectivamente. El rango de valores de dichos coeficientes se encuentra dentro del intervalo $[0,1]$ y deben satisfacer

$\omega_{\mathrm{u}}+\omega_{\mathrm{c}}=1$

Observando la ecuación (4-8), se aprecia que cada pareja de coeficientes de ponderación proporcionan al problema diferentes soluciones, $x_{r}$, que, al 
menos teóricamente, deberían pertenecer al conjunto óptimo de Pareto, y el correspondiente punto en el espacio objetivo, denominado $\left\{U\left(x_{r}\right), C\left(x_{r}\right)\right\}$ debería pertenecer a la frontera de Pareto de la Figura 4-2. De esta forma, el conjunto óptimo de Pareto de soluciones se puede obtener a partir de la optimización de la ecuación (4-8), simplemente cambiando los valores de los coeficientes de ponderación dentro del intervalo $[0,1]$, siempre que ambos coeficientes satisfagan la condición impuesta por la ecuación (4-9).

Por otra parte, los Algoritmos Genéticos tienen la característica intrínseca de trabajar en paralelo con varias posibles soluciones (individuos de la población). Usando estas características es posible obtener un conjunto externo de individuos constituidos por soluciones no dominadas encontradas durante el proceso de evolución. Este conjunto de soluciones no dominadas puede constituir una parte del conjunto óptimo de Pareto.

\subsubsection{AGMO basado en SPEA2}

El Strength Pareto Evolutionary Algorithm (SPEA) es una clase de AGMO perteneciente al conjunto de aproximaciones basadas en las fronteras de soluciones no dominadas para la búsqueda, o al menos aproximación, de la frontera óptima de Pareto.

En esta aproximación se busca minimizar la distancia a la frontera óptima y maximizar la diversidad de las soluciones generadas generación tras generación. El primer objetivo está directamente relacionado con la cuestión de cómo guiar la búsqueda hacia la frontera óptima de Pareto. El segundo objetivo viene determinado por los individuos que se deben mantener durante el proceso de evolución teniendo en cuenta la limitación de tiempo y recursos normales.

Para conseguir ambos objetivos, el SPEA combina tres técnicas en un único algoritmo: almacena externamente las soluciones óptimas de Pareto (Conjunto Externo), usa el concepto de dominancia de Pareto para asignar valores de la función objetivo y realiza agrupaciones para reducir el número de soluciones no-dominadas en la población externa (Selección Ambiental). La Figura 4-3 proporciona una vista esquemática de los pasos que constituyen este AGMO.

El algoritmo comienza con una población inicial de un tamaño dado (popsize). Esta población inicial o base puede ser proporcionada por el usuario (conjunto inicial) o generada aleatoriamente. Cada uno de estos individuos es una posible solución al problema de optimización. Este algoritmo genera una 
población auxiliar, de tamaño nrepl, constituido por los hijos obtenidos después del cruce y la mutación de ciertos individuos seleccionados de la población base. Los nuevos hijos generados se evalúan y se añaden a la población base. Cada individuo de la población resultante, compuesta por popsize+nrepl individuos, se penaliza si es necesario, y se escala para obtener un rango de individuos basados en su bondad.

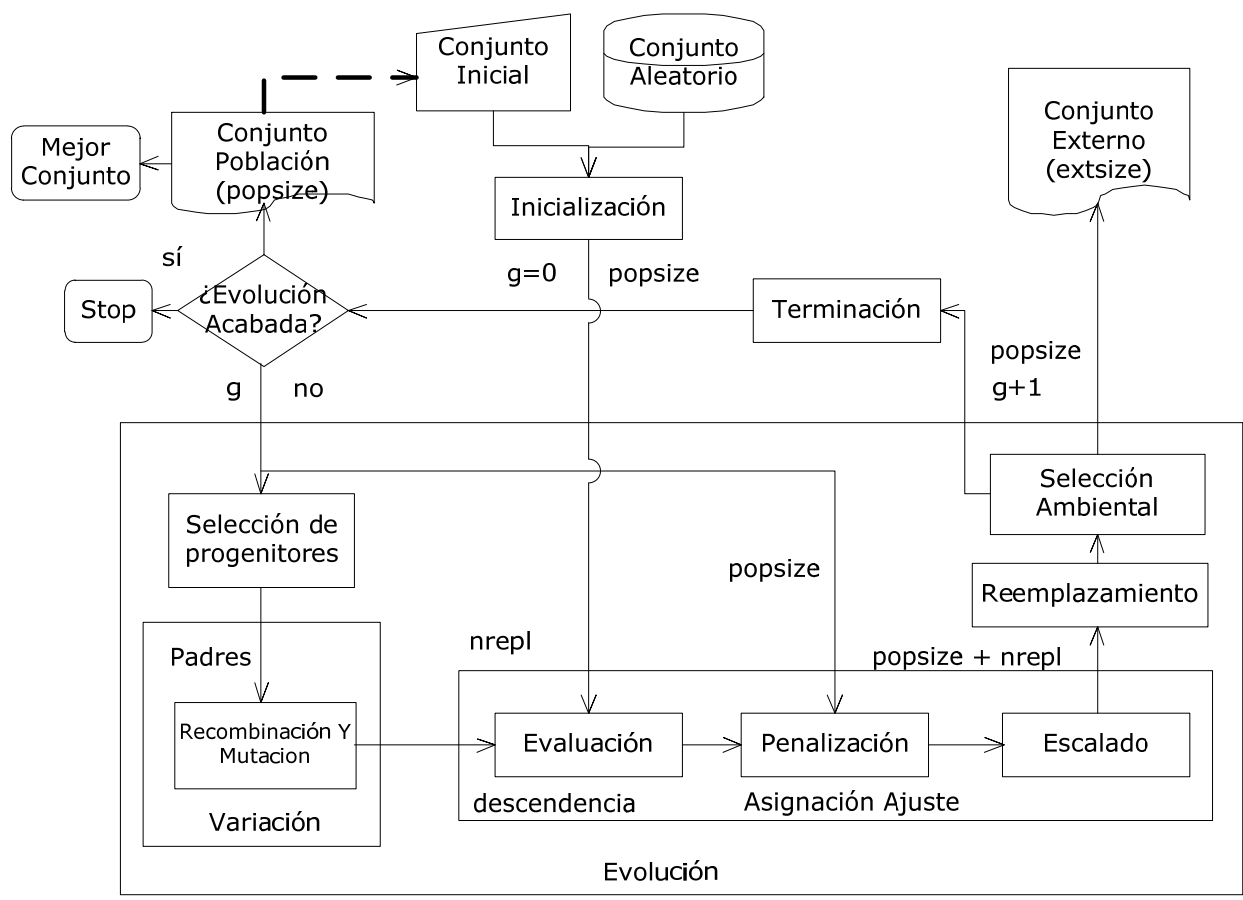

Figura 4-3: Principales componentes del AGMO basado en el SPEA2

La determinación del valor final de la función objetivo consiste en la asignación de una magnitud escalar a todos los individuos del conjunto de la población de la generación $g$ y del conjunto externo, representados por $P_{g}$ y $E_{g}$ respectivamente, para una generación dada, $g$, realizándose dicha asignación en pasos sucesivos. Primero, cada individuo, genéricamente denominado $i$, se le asigna un valor de fuerza $S(i)$, que representa el número de soluciones a las cuales domina. De acuerdo con este concepto de 
dominancia de Pareto, se dice que un individuo $j$, es un individuo nodominado por el individuo $i$, si:

1. La solución $j$ no es peor que $i$ para todos los criterios (p.e. $U(x)$ y $C(x)$ para el problema de $A[U]+C)$ y,

2. La solución $j$ es estrictamente mejor que $i$ en al menos uno de los criterios $(U(x)$ o $C(x)$ en el problema ejemplo).

Dados los supuestos anteriores, debe verificarse la expresión:

$S(i)=\left|\left\{j / j \in P_{g}+E_{g} \wedge i>j\right\}\right|$

donde el operador |.| indica la cardinalidad de un conjunto, ' + ' implica la unión de conjuntos y el símbolo ' > ' corresponde a la relación de dominancia de Pareto descrita anteriormente. En cualquier otro supuesto se dice que el individuo $j$ está dominado por el individuo $i$.

De esta forma se calcula para el individuo $i$ el total de las fuerzas de sus dominadores, como:

$R(i)=\sum_{j \in P_{g}+E_{g, j}>i} S(j)$

Obviamente, el objetivo es minimizar este valor, hasta $R(i)=0$ correspondiente a un individuo no dominado.

Con la finalidad de discriminar entre individuos que tengan igual valor de fuerza de sus dominadores, $R$, se incorpora información sobre la densidad. La estimación de la densidad de un individuo $i, D(i)$, se toma como la inversa de la distancia Euclídea a los k-ésimos individuos vecinos más cercanos, donde $k$ es normalmente:

$\mathrm{k}=\sqrt{\text { popsize }+ \text { extsize }}$

Para cada individuo $i$, las distancias, en el espacio objetivo, Figura 4-2, a todos los individuos pertenecientes a $P_{g}+E_{g}$ se calculan y almacenan en una lista. Después de ordenar la lista en orden creciente, el $k$-ésimo elemento proporciona la distancia solicitada, denotada como $\sigma_{i}^{g}$. El valor de la 
densidad, $D$ (i) se calcula como:

$\mathrm{D}(\mathrm{i})=\frac{1}{\sigma_{\mathrm{i}}^{\mathrm{g}}+2}$

la cual proporciona un valor final de la bondad del individuo $i, f(i)$ dado por:

$f(i)=S(i)+D(i)$

donde $S(i)$, representa a la fuerza del individuo $i$, calculada según la expresión (4-10) y $D(i)$ la densidad del mismo obtenida a partir de la ecuación (4-13).

A continuación, siguiendo el esquema de la Figura 4-3, el algoritmo verifica si se satisfacen las restricciones implícitas. En caso de violación de alguna de las restricciones, el valor de la función objetivo se penaliza. Así, el valor penalizado para un individuo dado, $i$, que viola alguna de las restricciones (asumiendo que el objetivo es minimizar) se formula como:

$F(i)=f(i)+[1-\mu(\alpha, \beta, \gamma)] f(w)$

donde $f(w)$ es el valor correspondiente al peor individuo encontrado en la población en la presente generación y $\mu(\alpha, \beta, \gamma)$ es la función penalización.

Después del cálculo del valor de la función objetivo y penalización, si es necesario, del mismo, se ordenan los individuos en función del valor penalizado y se eliminan del proceso de optimización los peores nrepl, de manera que el tamaño de la población vuelve a ser el original (popsize). Por tanto, después del reemplazamiento, los mejores individuos permanecen en la nueva población constituyendo la nueva generación, generalmente denominada $g+1$, la cual desciende de la previa, $g$.

El siguiente paso, ver Figura 4-3, lo constituye la Selección Ambiental, en la cual todos los individuos no-dominados en la nueva población se añaden al Conjunto Externo. De este conjunto externo extendido se eliminan los individuos que estén dominados, haciendo uso de la expresión (4-10). Si el tamaño final del conjunto externo de soluciones no dominadas (extsize) excede un tamaño máximo, el algoritmo lleva a cabo un agrupamiento para reducir las soluciones no-dominadas de la Población Externa. Después, el 
algoritmo comprueba si se cumple el criterio de finalización. Si no se satisface este criterio, la evolución continua para crear una nueva población, como se ha descrito con anterioridad, en caso contrario finaliza.

El algoritmo SPEA proporciona muy buenos resultados, en comparación con otros AGMOs, y ha sido usado en diferentes aplicaciones. SPEA2 es una versión mejorada, que incorpora una estrategia de asignación de ajuste de grano fino, una técnica de estimación de la densidad y un método de truncación de archivos destacados.

\subsection{Consideración de incertidumbres en la toma de decisiones}

\subsubsection{Principios de la toma de decisiones con incertidumbres.}

La incorporación adecuada de la incertidumbre en el análisis de un sistema complejo, es un tema importante y de gran interés. Se han propuesto muchos métodos para considerar los dos tipos de incertidumbres consideradas (aleatorias y epistémicas) y explicadas en el apartado 3.8. Por ejemplo (Apostolakis 1993) considera la combinación de modelos ponderados por el juicio de un analista mientras que (Zio 1996) propone el uso un factor de ajuste para el modelo más apropiado. En la literatura existe un abundante número de estudios que manejan la incertidumbre asociada a los modelos de cuantificación, p.e. para los problemas de optimización de fiabilidad, pruebas y mantenimiento (Rocco 2000), (Bunea 2002), (Marseguerra 2004a). (Marseguerra 2004b), (Coit 1998), (Coit 2002), (Marseguerra 2005)y (Martorell 2007). Por ejemplo, los cuatro primeros se enfrentan al problema de optimizar pruebas y mantenimientos basados en criterios de indisponibilidad y costes, trabajando con las incertidumbres asociadas con alguno de los parámetros del modelo como la tasa de fallo del equipo, probabilidad de fallo a la demanda, efectividad del mantenimiento, etc.

En general, se puede decir que los análisis llevados a cabo para soportar el proceso de toma de decisiones sobre mejora del diseño, de la vigilancia y del mantenimiento en base a criterios de confiabilidad y riesgo tratan el efecto de las incertidumbres de dos maneras distintas, dependiendo de si el analista es "neutral" o "adverso" al riesgo. Así, en el primer caso el proceso de toma de decisiones se basa en los valores esperados adoptando la estimación de valores medios para los criterios de decisión, mientras que en el segundo caso la utilización de valores medios se considera poco significativa si no es acompañada con medidas complementarias tales como varianzas, cotas superiores o inferiores, intervalos de tolerancia, intervalos de confianza, etc. 
A lo largo de esta última década han sido muchas las aplicaciones llevadas a cabo sobre la toma de decisiones en la mejora del diseño, de la vigilancia y el mantenimiento de equipos considerando la presencia de incertidumbres. Estas aplicaciones han demostrado que la inclusión del efecto de las incertidumbres condiciona de forma importante, el proceso de toma de decisiones, no sólo en cuanto a la necesidad de nuevos métodos sino también en cuanto a los resultados finalmente alcanzados.

La manera de incorporar el efecto de las incertidumbres en el proceso de toma de decisiones bajo la aproximación "adversa" al riesgo ha seguido dos direcciones. La primera formula el efecto de las incertidumbres como un objetivo más del problema de decisiones junto al resto de criterios. En este caso se persiguen buenas soluciones en las variables de la toma de decisiones (p.e. confiabilidad, riesgo y coste) que al mismo tiempo minimicen el efecto de las incertidumbres (Coit 1998). En cambio, la segunda dirección busca mantener dicho efecto bajo control, por ejemplo, fijando como objetivo minimizar una cota superior, o con la imposición de un valor máximo de incertidumbre como restricción (Coint 2004), (Marseguerra 2005), (Marseguerra 2004a), (Marseguerra 2004b). Ambas aproximaciones tiene sus ventajas e inconvenientes. La primera necesita menos esfuerzo computacional, pero se debe definir a priori cuál es el grado de "adversidad" al riesgo. La segunda permite tomar la decisión final en el conjunto óptimo de Pareto que muestra explícitamente los efectos de diferentes niveles de "adversidad" al riesgo en las soluciones óptimas, y de esta forma mantener bajo control las incertidumbres en el proceso de optimización.

Adoptando la aproximación "adversa" al riesgo para el proceso de toma de decisiones en presencia de incertidumbres, el problema de optimización multiobjetivo se plantea adaptando la formulación convencional introducida en la sección 4.1 y 4.2 donde los criterios $R A M S+C$ actúan como criterios de decisión y los parámetros de interés asociados con el diseño y/o las actividades de vigilancia y mantenimiento actúan como variables de decisión. Puesto que dichos parámetros están afectados de incertidumbre, la estimación de las funciones objetivo consideradas también lo estará. Puesto que los criterios de decisión forman parte tanto de los objetivos como de las restricciones al problema de optimización multiobjetivo, entonces diremos que nos enfrentamos a un problema de optimización con incertidumbre en los objetivos y en las restricciones.

La aproximación utilizada en esta tesis se basa en el mantener bajo control el impacto de la incertidumbre. Sin embargo, el control del efecto de las incertidumbres en los criterios de indisponibilidad y coste se puede introducir como una nueva restricción considerada como objetivo prioritario (fuerte), o bien mediante su inclusión mediante restricciones, débiles, dentro de las 
funciones objetivo del POMO.

Así, como se ve en (Martorell 2000), la satisfacción de las restricciones y la optimización multiobjetivo pueden ser vistas como dos aspectos del mismo problema. Ambas envuelven la optimización simultánea de un número de funciones. Así, las restricciones pueden ser vistas a menudo como objetivos prioritarios (fuertes), los cuales necesitan ser satisfechos antes de que la optimización del resto de objetivos, débiles, tenga lugar, o por otro lado, los problemas caracterizados por un número de objetivos débiles son a menudo reformulados como un problema de optimización con restricciones.

Es por ello que no se busca mantener la incertidumbre bajo un mínimo, sino que el objetivo es mantener simultáneamente bajo control la incertidumbre de todo el conjunto de criterios, por ejemplo la indisponibilidad y el coste o cualquier otro que se seleccione. Así, es posible mantener bajo control el efecto de la incertidumbre en los resultados de la optimización, lo que concuerda con los requisitos de la filosofía de la toma de decisiones informadas en el riesgo, y al mismo tiempo es posible reducir el esfuerzo computacional, lo cual es bastante deseable.

\subsubsection{Formulación del problema de optimización considerando incertidumbres}

Muchos de los métodos existentes de propagación de incertidumbres siguen una aproximación similar, basada en la estimación de la función de distribución de las incertidumbres en los criterios de decisión, después de generar una serie de parámetros iniciales de entrada, propagándolos mediante un modelo de cuantificación adecuado, y calculando los correspondientes limites superior e inferior de cada variable de decisión, o derivando percentiles, p.e. $5 \%$ y $95 \%$, medias, varianzas, etc. En general, es necesaria una cantidad ingente de casos para obtener una buena estimación de estos estadísticos.

Sin embargo, existen otros métodos de cuantificación de incertidumbres (Martorell 2007) que no requieren la estimación de la función de distribución de los criterios de decisión, por lo que el esfuerzo de cálculo es mucho menor, lo que facilitaría la posible integración en una aproximación híbrida entre los métodos evolutivos como los AA.GG. y los Montecarlo. Esta aproximación no-paramétrica permite desacoplar la asociación entre el número de parámetros con incertidumbre y el número de cálculos requeridos.

En la presente tesis se utiliza un método basado en la estimación de 
intervalos de tolerancia, explotando las ventajas de los estadísticos ordenados, para cuantificar la incertidumbre asociada a una optimización en la que las funciones objetivo son la confiabilidad y el coste.

\subsubsection{Intervalos de tolerancia para distribuciones libres o no paramétricas.}

Vamos a asumir que se llevan a cabo $N$ ejecuciones del modelo de cuantificación con parámetros de entrada variables usando un muestreo por Monte-Carlo simple, obteniéndose entonces una serie de estimaciones $Y_{1}, . ., Y_{N}$ de los criterios de decisión aleatorias, p.e. indisponibilidad o costes. Se ordenan los resultados en orden creciente de magnitud de forma $Y(1), \ldots, Y(N)$. A estos datos ordenados se les denomina estadísticos de orden. Si $Y(j)$ es el estadístico de orden que corresponde con la estimación $Y_{i}$, entonces el rango de $Y_{i}$ es $j$.

Los estadísticos de orden proporcionan un modo de estimación de la proporción de datos que podrían estar por encima o por debajo de un valor dado, llamado percentil. El percentil $\gamma$ es un valor, $Y(V)$, tal que al menos $\left(100^{*} \gamma\right) \%$ de las medidas son menores que este valor y al menos $100^{*}(1-\gamma$ ) $\%$ son mayores. Al percentil 50 se le denomina mediana.

Un intervalo de confianza cubre un parámetro de la población, p.e. media o varianza, con una confianza determinada, esto es, en un número determinado de veces.

Hay también una forma de cubrir una proporción fija de una población con un determinado nivel de confianza. A dicho intervalo se le denomina intervalo de tolerancia. Los extremos del intervalo se denominan límites de tolerancia, p.e. límite inferior $(L)$ y superior $(H)$ de tolerancia.

Los límites de confianza son límites dentro de los cuales se espera encontrar un valor poblacional determinado, tal como la media, mientras que los límites estadísticos de tolerancia son límites dentro de los cuales se espera encontrar una determinada proporción de la población. Los intervalos de confianza derivan hacia cero a medida que el tamaño muestral aumenta. Los intervalos de tolerancia tienden hacia un valor fijo a medida que el tamaño muestral se incrementa.

Los límites de tolerancia pueden obtenerse a partir de las $N$ estimaciones de la variable de salida o funciones objetivo $Y$, incluso cuando no se conoce la forma de la distribución de dichas variables. Usando uno o ambos límites de 
tolerancia es posible derivar un par de intervalos unilaterales de tolerancia de distribución libre o no paramétricos para las funciones objetivo [ $L,+\infty$ [ y ]$-\infty, H]$, y un intervalo bilateral de tolerancia de distribución libre 0 no paramétricos, p.e. [L,H].

El tamaño muestral, $N$, condiciona los límites de tolerancia, por lo que resulta de interés conocer el tamaño muestral mínimo que garantice con una probabilidad $\beta$ que el intervalo de tolerancia contenga al menos una proporción $\boldsymbol{V}$ de la población total.

Muchos autores han discutido sobre el problema de establecer unos límites de tolerancia para una distribución a través de las muestras observadas. Sin embargo, resolver este problema cuando no se conoce nada sobre la función de densidad excepto el hecho de su continuidad, no parece una tarea fácil. Wilks (Wilks 1941) llevó a cabo los primeros trabajos al respecto, explotando las ventajas de los estadísticos ordenados y más tarde Robbins (Guba 2003), probó que los límites de tolerancia de una distribución libre pueden obtenerse solo mediante estadísticos ordenados.

\subsection{Intervalo de tolerancia unilateral para una función objetivo}

Sean los estadísticos de orden $Y(1), Y(2), \ldots, Y(N)$ que representan los datos ordenados en orden creciente de las estimaciones $Y_{1}, Y_{2}, \ldots, Y_{N}$ de una función objetivo $Y$, p.e. indisponibilidad o costes obtenidos a partir de una simulación con Montecarlo con muestreo aleatorio simple. Por definición se establece que $Y(0)=-\infty$ e $Y(N+1)=+\infty$.

En este caso para algunos $\gamma<1$ y $\beta<1$ positivos se pueden construir dos funciones aleatorias $L\left(Y_{1}, Y_{2}, \ldots, Y_{N}\right)$ y $H\left(Y_{1}, Y_{2}, \ldots, Y_{N}\right)$ llamadas límites de tolerancia tales que

$\operatorname{Pr}\left\{\int_{\mathrm{L}}^{\mathrm{H}} \mathrm{g}(\mathrm{Y}) \mathrm{dY}>\gamma\right\}=\beta$

donde 
$\int_{L}^{H} g(Y) d Y$

es una variable aleatoria, que representa la proporción de la distribución que se encuentra contenida en el intervalo $[L, H]$. A la probabilidad $\beta$ se le conoce como nivel de confianza.

Fijado $\beta$ y $\gamma$ en el intervalo $[0,1]$, es posible determinar a partir de (4-16) el tamaño muestral necesario para asegurar el intervalo de tolerancia que represente un cobertura $\boldsymbol{V}$ del domino de resultados con un nivel de confianza $\beta$ (4-18).

$\beta=\sum_{j=0}^{s-r-1}\left(\begin{array}{l}N \\ j\end{array}\right) \gamma^{j}(1-\gamma)^{N-j}$

donde $r$ y $s$ cumplen que $0 \leq r \leq s \leq N$, y son los rangos que tomamos para los límites inferior y superior para nuestro intervalo de confianza, $L=Y(r)$, $H=Y(s)$. Remarcar que $\gamma$, no es una probabilidad aunque es un número no negativo no mayor a 1 .

La selección de los límites de tolerancia $L=Y(1)$, p.e. $r=1$, y $H=Y(N)$, p.e. $s$ $=N$, es, en muchas ocasiones, la forma de determinar el intervalo de tolerancia bilateral $[L, H]=[Y(1), Y(N)]$. Sin embargo, cuando solamente estamos interesados en el límite superior de tolerancia $H=Y(N)$, entonces llamamos a ese intervalo $[Y(0), Y(N)]$, p.e. $]-\infty, H]$, intervalo de tolerancia unilateral inferior, o alternativamente cuando se está interesado en el límite inferior de tolerancia $L=Y(1)$, también podemos llamar al intervalo $[Y(1)$, $Y(N+1)]$, p.e. [L, $+\infty$ [, intervalo de tolerancia unilateral superior. Sustituyendo ahora en la expresión (4-18) por ejemplo $r=0$ y $s=N$, o alternativamente $r=$ 1 y $s=N+1$, se obtiene la siguiente expresión

$\beta=1-\gamma^{\mathrm{N}}$

A esta ecuación (4-19) se le denomina fórmula de Wilks para una única variable y un límite de tolerancia unilateral, y se usa para determinar $Y(1)$ o $Y(N)$. Esta ecuación puede reescribirse para obtener el tamaño muestral en función de $\beta$ y $\gamma$, como: 
$\mathrm{N}=\frac{\ln (1-\beta)}{\ln (\gamma)}$

La Tabla 4-1 presenta el número de muestras $N$ necesario para obtener un intervalo de tolerancia unilateral para una función objetivo obtenido a partir de diferentes pares de niveles de tolerancia/confianza $\gamma / \beta$.

Tabla 4-1: Tamaño muestral $\mathbf{N}$ para una variable e intervalo unilateral.

\begin{tabular}{ccccc}
\hline & \multicolumn{4}{c}{ Nivel de tolerancia( Y ) } \\
\hline & & 0.90 & 0.95 & 0.99 \\
Nivel de & 0.90 & 22 & 45 & 230 \\
confianza & 0.95 & 29 & 59 & 299 \\
$(\boldsymbol{\beta})$ & 0.99 & 44 & 90 & 459 \\
\hline
\end{tabular}

Así, después de $N$ muestreos de una función o variable objetivo de un modelo con variables o parámetros de entrada fluctuantes, usando un muestreo por Montecarlo, seleccionamos el valor más alto de los resultados del muestreo $H\{Y\}_{Y / \beta}=Y(N)$, que será el límite superior $\left.]-\infty, H\{Y\}_{Y / \beta}\right]$, el cual representa una cobertura del dominio de resultados por encima del correspondiente nivel de tolerancia/confianza $\gamma / \beta$. Alternativamente, también se puede seleccionar el valor más bajo de las funciones muestreadas, $L\{Y\}_{Y / \beta}$ $=Y(1)$, que será el límite inferior $\left[L\{Y\}_{Y / \beta},+\infty[\right.$, el cual representa una cobertura del dominio de resultados por debajo del correspondiente nivel de tolerancia/confianza $(1-\gamma) / \beta$. En general se utiliza la función $T\{Y\}_{Y / \beta}$ tanto para denotar el valor más alto, $T\{Y\}_{Y / \beta}=H\{Y\}_{Y / \beta}$, como el más bajo, $T\{Y\}_{Y / \beta}=L\{Y\}_{Y / \beta}$, de los límites de tolerancia asociados a los intervalos de tolerancia unilaterales.

La Figura 4-4 muestra las probabilidades $\beta$ frente a $N$ para cuatro valores de $\gamma$. Analizando dicha figura se observa que con un número mayor de muestreos, la curva de probabilidades $\beta$ alcanza la saturación y tiende a la unidad a medida que $N$ crece. A medida que disminuyen los valores de $\gamma$, se alcanza antes la saturación en las curvas de probabilidades, ya que un menor $\gamma$, implica que se requiere que una menor fracción de las muestras se 
encuentre en un intervalo dado. Un valor de $y$ pequeño no es aceptable en algunos casos, p.e. desde el punto de vista de la disponibilidad y el coste, ya que un valor pequeño de $y$ implica que una gran proporción de valores de la función objetivo pueden caer fuera del intervalo de tolerancia, por lo que prácticamente se hace necesario valores de $y>0.9$.

Por otro lado, señalar que $H\{Y\}_{Y / \beta \rightarrow 1}$ mantiene un percentil $\gamma$ de la función o variable $Y$, mientras que $L\{Y\}_{Y / \beta \rightarrow 1}$ mantiene el percentil $(1-\gamma)$ de la misma. Sin embargo, y como consecuencia del comportamiento asintótico de la ecuación (4-19), se requiere una gran cantidad de muestreos para obtener ambos percentiles (p.e. $\beta \rightarrow 1$ ) especialmente cuando $y>0.9$.

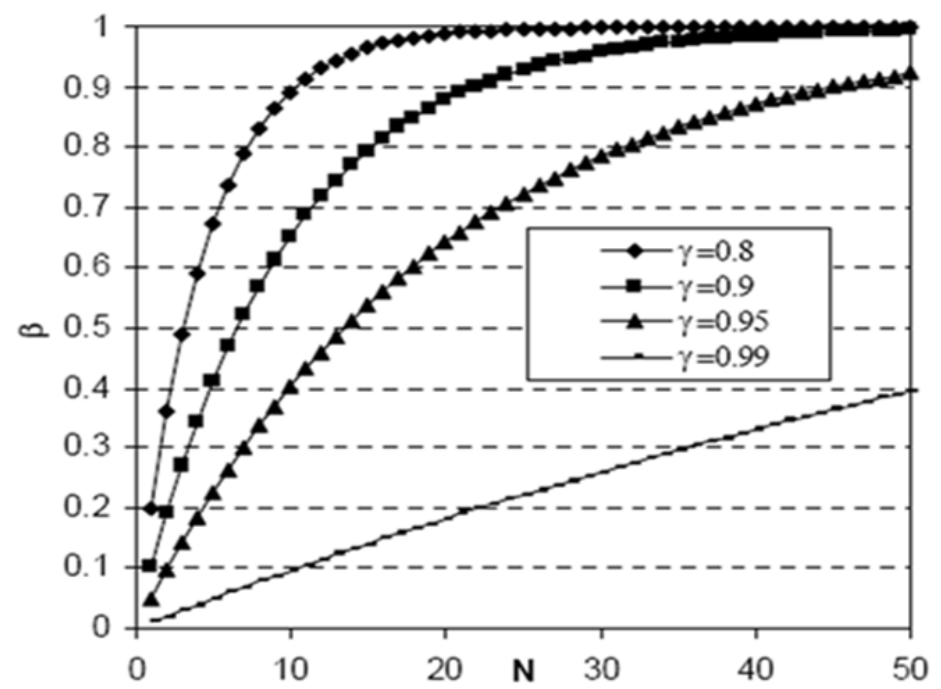

Figura 4-4: Dependencia del nivel de confianza $\beta$ respecto al número de muestras con varios niveles de tolerancia y para el intervalo de tolerancia unilateral.

\subsection{Intervalo de tolerancia unilateral para dos funciones objetivo.}

Consideremos el caso de varias funciones objetivo, que para simplificar tomaremos el par formado por la indisponibilidad, $\mathrm{U}, \mathrm{y}$ el coste, $\mathrm{C}$, dentro del caso $A(U)+C$, ya que su extensión a un mayor número de funciones dentro del RAMS $+C$ es directa. 
La ecuación (4-21) muestra una matriz con las estimaciones para la indisponibilidad, $U$, como primera fila, y coste, $C$, en la segunda fila, obtenidos a partir de una simulación mediante muestreo por Montecarlo simple. La secuencia de filas en la matriz de muestreo $M$ puede ser arbitraria.

$\mathrm{M}=\left(\begin{array}{llll}\mathrm{U}_{1} & \mathrm{U}_{2} & \ldots & \mathrm{U}_{\mathrm{N}} \\ \mathrm{C}_{1} & \mathrm{C}_{2} & \ldots & \mathrm{C}_{\mathrm{N}}\end{array}\right)$

El problema de determinar los límites de tolerancia para ambas variables $U$ y $\mathrm{C}$ ha de tener en cuenta la dependencia entre ambas variables. La consideración de la dependencia temporal tiene influencia en el tamaño muestral requerido por lo que en otros trabajos se ha asumido la independencia entre variables como en (Martorell 2007).

Sean los estadísticos ordenados $U(1), \ldots, U(N)$, los datos ordenados en orden creciente de una serie de estimaciones de la indisponibilidad tomados de la primera fila de la matriz $M$. Se seleccionan de estos $U\left(r_{U}\right)$ y $U\left(s_{U}\right)>U\left(r_{U}\right)$ como $L_{U}$ y $H_{U}$ respectivamente (límites inferior y superior de los intervalos de confianza para la variable $U$ ). Sean $i_{1}, i_{2}, \ldots, i_{s-r-1}$ que representan los índices de columna originales de los elementos $U\left(r_{U}+1\right), U\left(r_{u}+2\right), \ldots, U\left(r_{U}+s_{U}-1\right)$. A continuación se escogen de la segunda fila que representa las $N$ estimaciones de costes y se ordenan la parte $\mathrm{Ci}_{1}, \mathrm{Ci}_{2}, \ldots, \mathrm{Ci}_{\mathrm{SU}-\mathrm{rU}-1}$ de sus elementos en orden creciente para obtener $C(1)<C(2)<\ldots<C\left(s_{U}-r_{U}-1\right)$. De entre estos se seleccionan $C\left(r_{C}\right)$ y $C\left(s_{C}\right)>C\left(r_{C}\right)$ como $L_{C}$ y $H_{C}$, respectivamente. Puede entonces definirse un volumen bidimensional de la forma

$\left\{\left[L_{U}, H_{U}\right],\left[L_{C}, H_{C}\right]\right\}$

Para determinar unos límites de tolerancia unilaterales en el supuesto que estamos tratando, se recomendaría la elección de $r_{U}=r_{C}=0$ y $s_{C}=s_{U}=N-1$. En la práctica, esto significa seleccionar justo el estimador para la indisponibilidad más bajo, $L_{U}=U(1)$ o el más alto $H_{U}=U(N)$, eliminar las correspondientes columnas con el valor de indisponibilidad más alto o más bajo de $\mathrm{M}$, y entonces ordenar las estimaciones de costes que se mantienen $C(1)<C(2)<\ldots<C(N-1)$ y seleccionar aquella con el valor de coste más bajo, $L_{C}=C(1)$, o más alto, $H_{C}=C(N-1)$. En (Guba 2003) se propone el uso de (4-23) para determinar el mínimo tamaño muestral $N$ necesario para obtener unos límites de tolerancia unilaterales utilizando el procedimiento anterior para $p \geq 2$ variables dependientes 
$\beta=\sum_{j=0}^{N-p}\left(\begin{array}{c}N \\ j\end{array}\right) \gamma^{j}(1-\gamma)^{N-1}$

Dicha expresión garantiza un nivel de tolerancia, $\boldsymbol{Y}$, en el dominio de resultados con un nivel de confianza, $\beta$. La ecuación (4-23) se simplifica para el caso particular con dos variables dependientes, p.e. indisponibilidad y coste, $p=2$, como sigue

$\beta=1-\gamma^{\mathrm{N}}-(\mathrm{N}-1)(1-\gamma) \gamma^{\mathrm{N}-1}$

La Tabla 4-2 detalla el tamaño muestral necesario para obtener un intervalo de tolerancia unilateral para dos funciones objetivo dependientes a partir de la ecuación (4-24).

Tabla 4-2: Tamaño muestral $\mathbf{N}$ para dos funciones objetivo e intervalo unilateral.

\begin{tabular}{ccccc}
\hline & \multicolumn{5}{c}{ Nivel de tolerancia ( Y ) } \\
\hline Nivel de & $\mathbf{0 . 9 0}$ & $\mathbf{0 . 9 0}$ & $\mathbf{0 . 9 5}$ & $\mathbf{0 . 9 9}$ \\
confianza ( $\boldsymbol{\beta}$ ) & $\mathbf{0 . 9 5}$ & 38 & 77 & 388 \\
& $\mathbf{0 . 9 9}$ & 46 & 93 & 473 \\
& & 64 & 130 & 662 \\
\hline
\end{tabular}

Así, después de $N$ muestreos de las funciones, para el caso $A[U]+C, U$ y $C$ a partir de un modelo de cuantificación con variables o parámetros de entrada fluctuantes, obtenemos el punto límite $\{U, C\}$ dado por $T\{U, C\}_{Y / \beta}$. Este se puede calcular obteniendo primero $T\{U\}_{Y / \beta}$. seleccionando el resultado más grande de la muestra, $H\{U\}_{Y / \beta}=U(N)$, como límite superior $\left.]-\infty, H\{U\}_{Y / \beta}\right]$, o el resultado más bajo, $L\{U\}_{Y / \beta}=U(1)$, como límite inferior $\left[L\{U\}_{Y / \beta},+\infty\right.$ [. Se elimina la pareja $\left\{U_{k}, C_{k}\right\}$ correspondiente al punto $U_{k}$ seleccionado como valor más bajo o más alto previamente. Se obtiene entonces $T\{C\}_{Y / \beta}$ de las muestras restantes $N-1$ seleccionando el resultado más grande de la muestra, $H\{C\}_{Y / \beta}=$ $C(N-1)$, como límite superior $\left.]-\infty, H\{C\}_{Y / \beta}\right]$, o el resultado más bajo, $L\{C\}_{Y / \beta}=C(1)$, como límite inferior $\left[L\{C\}_{Y / \beta},+\infty[\right.$. 
En general el límite de tolerancia puede ser formulado para el caso de dos funciones objetivo dependientes $U$ y $C$ como un límite bidimensional representado por

$\mathrm{T}\{\mathrm{U}, \mathrm{C}\}_{\gamma / \beta}$

el cual puede ser deducido siguiendo el proceso anterior, o alternativamente, para el caso de variables o funciones objetivo independientes, donde queda simplificado como:

$\mathrm{T}\{\mathrm{U}, \mathrm{C}\}_{\gamma / \beta}=\left\{\mathrm{T}\{\mathrm{U}\}_{\gamma / \beta}, \mathrm{T}\{\mathrm{C}\}_{\gamma / \beta}\right\}$

\subsubsection{Formulación AGMO y AGSO usando límites de tolerancia}

En este apartado se introduce la aproximación AGMO y AGSO basada en la utilización de intervalos de tolerancia para distribuciones libres o no paramétricas incorporando la incertidumbre dentro de la optimización. Se usa un procedimiento de simulación basado en Montecarlo para dichos sistemas y se explotan las ventajas de los estadísticos de orden para proporcionar intervalos de tolerancia de distribuciones libres o no paramétricas para la estimación de los criterios $R A M S+C$, basado en el número mínimo de simulaciones o muestreos $(N)$ necesario para garantizar un probabilidad de cobertura o tolerancia $(\gamma)$ con un nivel de confianza $(\beta)$. Para ello, nos centraremos en la aproximación basada en algoritmos genéticos introducida previamente, modificada para permitir la búsqueda considerando las incertidumbres de las funciones objetivo y las restricciones.

\subsection{Aproximación AGMO-H y AGMO-E}

El problema de optimización multiobjetivo general puede particularizarse y formularse en términos, por ejemplo, de $A[U]+C$, indisponibilidad más costes, basado en el método de intervalos de tolerancia para minimizar el vector de funciones multiobjetivo con incertidumbres,

$\mathrm{f}(\mathrm{x})=\mathrm{T}\{\mathrm{U}(\mathrm{X}), \mathrm{C}(\mathrm{X})\}_{\gamma / \beta}$

sujeto al vector de restricciones con incertidumbres 
$\mathrm{g}(\mathrm{x})=\left\{\mathrm{T}\{\mathrm{U}(\mathrm{X}), \mathrm{C}(\mathrm{X})\}_{\gamma / \beta}<\left\{\mathrm{U}_{\mathrm{L}}, \mathrm{C}_{\mathrm{L}}\right\}\right\}$

que para el caso de considerar, por ejemplo, únicamente los límites más altos de tolerancia se puede simplificar a

$\mathrm{f}(\mathrm{x})=\left\{\mathrm{H}\{\mathrm{U}(\mathrm{X})\}_{\gamma / \beta}, \mathrm{H}\{\mathrm{C}(\mathrm{X})\}_{\gamma / \beta}\right\}$

sujeto al vector de restricciones con incertidumbres

$\mathrm{g}(\mathrm{x})=\left\{\mathrm{H}\{\mathrm{U}(\mathrm{x})\}_{\gamma / \beta}<\mathrm{U}_{\mathrm{L}}, \mathrm{H}\{\mathrm{C}(\mathrm{x})\}_{\gamma / \beta}<\mathrm{C}_{\mathrm{L}}\right\}$

donde $x$ representa el vector de variables de decisión, en el que, por ejemplo, puede considerarse los intervalos de prueba y mantenimiento, y $U(x)$ y $C(x)$ representan la indisponibilidad y costes respectivamente. En la ecuación (4-30), se deben adoptar valores umbrales apropiados, p.e. $U_{L}=L\left\{U_{i}\right\}_{Y / \beta}$ y $C_{L}$ $=L\left\{C_{i}\right\}_{Y / \beta}$, donde $C_{i}$ y $U_{i}$ representan la estimación de costes $\mathrm{e}$ indisponibilidades respectivamente para la política actual de pruebas y mantenimiento, p.e. para los valores iniciales asignados a las variables de decisión. Alternativamente, se podría adoptar el percentil 5\% para la indisponibilidad $U_{L}=L\left\{U_{i}\right\}_{0.95 / 1}=p c t i l 5\left(U_{i}\right)$ y para el coste $C_{L}=L\left\{C_{i}\right\}_{0.95 / 1}=$ pctil5 $\left(C_{i}\right)$ en vez de los límites considerados.

Adicionalmente, se puede explorar una formulación alternativa, que hibride el uso de los valores medios y los límites de tolerancia superiores para los criterios de decisión en cuanto a la minimización de las siguientes funciones multiobjetivo con incertidumbres

$\mathrm{f}(\mathrm{x})=\left\{\mathrm{E}\{\mathrm{U}(\mathrm{X})\}_{\gamma / \beta}, \mathrm{E}\{\mathrm{C}(\mathrm{x})\}_{\gamma / \beta}\right\}$

sujeto al mismo vector de restricciones con incertidumbres dados por (4-30), donde las funciones multiobjetivo con incertidumbres se formulan en términos de valores medios para la indisponibilidad y el coste, p.e. $E\{U(x)\}_{Y / \beta}$ y $E\{C(x)\}_{Y / \beta}$ respectivamente, mientras las funciones de restricción permanecen igual basadas en los límite de tolerancia superior de ambos, para mantener bajo control el efecto de la incertidumbre en los resultados de la optimización, lo cual se muestra de acuerdo con la filosofía de la toma de decisiones informada en el riesgo. 


\subsection{Aproximación AGSO}

La aproximación aportada en (Martorell 2004) basada en el WEBA (Weighted Effectiveness Based Approach) permite transformar el problema de optimización multiobjetivo original en un número de problemas de optimización simple objetivo a resolver de forma secuencial, lo cual proporciona un número de soluciones cercanas a/o pertenecientes a la frontera óptima de Pareto. Así, usando la aproximación WEBA, las funciones objetivo y restricciones del AGSO, para el caso particular de considerar como criterios a indisponibilidad y el coste, pueden ser formulada basadas en el método de intervalos de tolerancia para minimizar la función escalar:

$f(x)=W(U(x), C(x))=1-\left(\omega_{u} \cdot e_{u}(x)+\omega_{c} \cdot e_{c}(x)\right)$

sujeta al mismo vector de restricciones con incertidumbres dado por (4-30). $\omega_{u}$ y $\omega_{c}$, son dos coeficientes de ponderación que introducen alguna flexibilidad en la búsqueda de la mejor solución que minimice (4-32), representando la importancia que se les asigna a las funciones de indisponibilidad y coste respectivamente. El rango de valores de dichos coeficientes se encuentra dentro de $[0,1]$ y deben satisfacer:

$\omega_{\mathrm{u}}+\omega_{\mathrm{c}}=1$

La siguiente expresión se puede usar para considerar la indisponibilidad del sistema y el coste respectivamente:

$e_{u}(x)=\frac{H\left\{U_{r}\right\}_{\gamma / \beta}-H\{U\}_{\gamma / \beta}}{H\left\{U_{r}\right\}_{\gamma / \beta}-H\left\{U_{m}\right\}_{\gamma / \beta}}$

$e_{c}(x)=\frac{H\left\{C_{r}\right\}_{\gamma / \beta}-H\{C\}_{\gamma / \beta}}{H\left\{C_{r}\right\}_{\gamma / \beta}-H\left\{C_{m}\right\}_{\gamma / \beta}}$

Hay dos situaciones singulares y extremas correspondientes con la adopción de la indisponibilidad como única función objetivo o en su caso, el coste como única función objetivo, lo cual puede formularse a partir de (4-32) seleccionando unos coeficientes de peso, $\omega_{u}$ y $\omega_{c}$, en sus valores extremos 1 y 0 , o alternativamente, 0 y 1 respectivamente. Ambas situaciones extremas son equivalentes a formular los siguientes dos POSO, p.e. para minimizar 
respectivamente las funciones simple objetivo

$\mathrm{f}(\mathrm{x})=\left\{\mathrm{H}\{\mathrm{U}(\mathrm{x})\}_{\gamma / \beta}\right\}$

denominada aproximación AGSO-U, y

$\mathrm{f}(\mathrm{x})=\left\{\mathrm{H}\{\mathrm{C}(\mathrm{x})\}_{\gamma / \beta}\right\}$

denominada aproximación AGSO-C. 


\section{Optimización multiobjetivo de requisitos STI y AOT a nivel de sistema y planta}

\subsection{Descripción del problema}

En esta aplicación se propone la optimización multiobjetivo de los requisitos de vigilancia y mantenimiento (STI y AOT) del sistema de generadores diésel de emergencia (GDE) de una central nuclear. Este sistema pertenece al sistema de suministro de energía alterna de emergencia de una central nuclear. El sistema de generadores diesel de emergencia se encuentra normalmente en espera y consiste en dos trenes redundantes, cada uno de ellos provisto de un generador diésel. Las especificaciones técnicas de funcionamiento establecen para dicho sistema un intervalo entre pruebas (STI) de 31 días (probando un tren de los generadores diésel cada 15 días) y un tiempo máximo de indisponibilidad (AOT) de $72 \mathrm{~h}$. Además, en aras de la simplicidad, en este caso de aplicación sólo se considera una actividad de mantenimiento preventivo, la cual se realiza anualmente y devuelve al equipo a una situación tan buena como si fuera nuevo (GAN). Se asume también que las actividades de pruebas y mantenimiento a optimizar se ejecutan online, esto es, mientras la central se encuentra en funcionamiento a potencia, por tanto, se han adoptado los modelos y datos derivados del APS a plena potencia para los cálculos de riesgos en la central nuclear.

En la siguiente sección se formula y resuelve la toma de decisiones multicriterio para cambios en los intervalos entre pruebas, mantenimiento 
preventivos y tiempos máximos permitidos de indisponibilidad basados en criterios RAMS+C.

\subsection{Formulación del problema}

Los criterios RAMS $+C$ se han formulado teniendo en cuenta los supuestos anteriores usando las expresiones introducidas en el capítulo 3 y con los datos de la Tabla 5-1 y Tabla 5-2. Estos modelos dependen explícitamente de los parámetros básicos relacionados con pruebas y mantenimientos candidatos para actuar como variables de decisión en la toma de decisiones multicriterio. Las variables de decisión relacionadas con pruebas y mantenimientos consideradas para este caso de aplicación se han codificado en el siguiente vector de decisión $x$ :

$\mathrm{x}=\{\mathrm{T}, \mathrm{M}, \mathrm{D}\}$

donde $T$, representa el tiempo entre pruebas o STI, $M$ el periodo del mantenimiento preventivo y $D$, el tiempo máximo fuera de servicio para un generador diésel o AOT.

Se define el vector de funciones objetivo, $f(x)$, que consiste en los criterios de aceptación relevantes para la toma de decisiones basadas en los atributos RAMS+C. En la presente aplicación se considera el siguiente vector objetivo:

$\mathrm{f}(\mathrm{x})=\left\{\mathrm{u}_{\mathrm{r}}(\mathrm{x}), \mathrm{u}_{\mathrm{tmc}}(\mathrm{x}), \Delta \mathrm{R}_{\mathrm{GDE}}(\mathrm{x}), \mathrm{r}_{\mathrm{GDE}}(\mathrm{x}), \mathrm{R}_{\mathrm{GDE}}(\mathrm{x}), \mathrm{C}_{\mathrm{GDE}}(\mathrm{x})\right\}$

donde $u_{r}(x)$ es la indisponibilidad asociada a la fiabilidad de uno de los generadores diesel de emergencia (3-11), $u_{t m c}(x)$ es la indisponibilidad de uno de los generadores diesel de emergencia debido a pruebas y mantenimiento (3-12) y (3-13), $\Delta R_{G D E}$, es el incremento condicional del riesgo con un generador diesel de emergencia fuera de servicio (por ejemplo debido a pruebas, mantenimiento correctivo, etc.), $r_{G D E}(x)$ es el riesgo simple por fallo de un generador diesel de emergencia (3-15), $R_{G D E}(x)$ es la contribución anual al riesgo por un generador diesel de emergencia fuera de servicio (3-16), y finalmente $C_{G D E}(x)$ es el coste total asociado a un cierto nivel de RAMS calculado para el sistema de generadores diesel de emergencia ((3-17) a (3-20)). Las expresiones de cada una de las funciones objetivo se ha expuesto en el capítulo 3 de la presente tesis. 
En la Tabla 5-1 se muestran los parámetros que intervienen en los modelos de fiabilidad, mantenibilidad, disponibilidad, y seguridad del capítulo 3 y que aplican al presente caso.

Tabla 5-1: Datos de fallos para generadores diesel de emergencia.

\begin{tabular}{|c|c|c|c|}
\hline Descripción & Parámetro & Valor & Unidad \\
\hline \multicolumn{4}{|l|}{ Fallo } \\
\hline Tasa de fallo a la espera residual & $\lambda_{0}$ & $4,19 \mathrm{E}-6$ & $h^{-1}$ \\
\hline Probabilidad de fallo por demanda & $\rho$ & $5,32 E-4$ & \\
\hline $\begin{array}{l}\text { Frecuencia mantenimiento residual (fallo en } \\
\text { espera) }\end{array}$ & $\omega_{0}$ & $4,19 \mathrm{E}-6$ & $\mathrm{~h}^{-1}$ \\
\hline $\begin{array}{l}\text { Probabilidad de mantenimiento (fallo a la } \\
\text { demanda) }\end{array}$ & C & $5,32 \mathrm{E}-4$ & \\
\hline Tasa de Fallo en misión & $\lambda_{\mathrm{M}}$ & $1,83 E-4$ & $\mathrm{~h}^{-1}$ \\
\hline \multicolumn{4}{|l|}{ Degradación } \\
\hline Tasa de envejecimiento lineal & $\alpha$ & $4,57 \mathrm{E}-10$ & $\mathrm{~h}^{-2}$ \\
\hline \multicolumn{4}{|l|}{ Pruebas } \\
\hline Intervalos entre pruebas & STI o T & 1440 & $\mathrm{~h}$ \\
\hline Tiempo medio de prueba & $\mathrm{T}$ & 6 & $\mathrm{~h}$ \\
\hline Tiempo medio funcionamiento & $t_{M}$ & 6 & $\mathrm{~h}$ \\
\hline \multicolumn{4}{|l|}{ Mantenimiento } \\
\hline $\begin{array}{l}\text { Tiempo máximo permitido fuera de } \\
\text { funcionamiento }\end{array}$ & AOT o D & 72 & $\mathrm{~h}$ \\
\hline $\begin{array}{l}\text { Tiempo medio fuera de servicio } \\
\text { por reparación }\end{array}$ & $\mu$ & 15 & $\mathrm{~h}$ \\
\hline Mantenimiento preventivo medio & $\sigma$ & 7,2 & $\mathrm{~h}$ \\
\hline Intervalos mantenimiento preventivo & M & 8760 & $\mathrm{~h}$ \\
\hline
\end{tabular}

La Tabla 5-2 muestra los parámetros que se han tomado para los modelos de costes expuestos en el capítulo 3 y que concurren en el caso de aplicación 
presente.

Tabla 5-2: Datos de costes para generadores diesel de emergencia.

Descripción

\section{Pruebas}

Coste horario debido a pruebas

Mantenimiento

Coste horario debido a mantenimiento correctivo

Coste horario debido a mantenimiento preventivo

Coste por reemplazamiento

\section{Fuera de servicio}

Coste por caída del equipo

\section{Accidente}

Coste por accidente
Parámetro Valor Unidad

$c_{\text {ht }} \quad 200 \quad € / h$

Chc $1200 € / h$

$\mathrm{C}_{\mathrm{hm}}$

$800 € / h$

$\mathrm{c}_{\mathrm{r}} \quad 36000 €$

$\mathrm{C}_{\mathrm{u}} \quad 2 \mathrm{E} 6 \quad €$

Cacc 2 E9 $€$

Además, se define un vector de funciones de restricción, $g(x)$, para formular los objetivos numéricos de acuerdo a los criterios de aceptación involucrados en la toma de decisiones RAMS+C. Aquí, el vector de restricción se ha formulado de acuerdo con los objetivos numéricos sugeridos en la sección 2.5, (RG 1174) y (RG 1177), lo cual puede representarse como:

$$
\begin{gathered}
\mathrm{g}(\mathrm{x})=\left\{\mathrm{u}_{\mathrm{r}}(\mathrm{x}) \leq 0,05, \mathrm{u}_{\mathrm{tmc}}(\mathrm{x}) \leq 0,02 ; \Delta \mathrm{R}_{\mathrm{GDE}}(\mathrm{x}) \leq 1,0 \mathrm{E}-3 ;\right. \\
\mathrm{r}_{\mathrm{GDE}}(\mathrm{x}) \leq 5,0 \mathrm{E}-7 ; \mathrm{R}_{\mathrm{GDE}}(\mathrm{x})-\mathrm{R}_{\mathrm{GDE}}\left(\mathrm{x}_{\mathrm{i}}\right) \leq 1,0 \mathrm{E}-6 ; \\
\left.\mathrm{C}_{\mathrm{GDE}}(\mathrm{x})-\mathrm{C}_{\mathrm{GDE}}\left(\mathrm{x}_{\mathrm{i}}\right) \leq 0\right\}
\end{gathered}
$$

donde $x_{i}$ representa el vector de decisión inicial, cuyo valor se encuentra en la Tabla 5-3. 
Tabla 5-3: Restricciones en las variables de decisión

\begin{tabular}{llll}
\hline Parámetro & Inicial & Paso & Rango de valores permitidos \\
\hline $\mathbf{T}$ & $720 \mathrm{~h}$ & $24 \mathrm{~h}$ & $168 \mathrm{~h} \leq T \leq 8760 \mathrm{~h}$ \\
$\mathbf{M}$ & $8760 \mathrm{~h}$ & 0,5 años & 0,5 años $\leq L \leq 40$ años \\
D & $72 \mathrm{~h}$ & $1 \mathrm{~h}$ & $15 \mathrm{~h} \leq D \leq 168 \mathrm{~h}$ \\
\hline
\end{tabular}

Además, en dicha tabla, se propone un rango de valores posibles de las variables de decisión $T, M$ y $D$ en los que se satisfacen las restricciones explícitas. Estas restricciones podrían representar por ejemplo valores tipificados como buenas soluciones para ser implementadas en la planta debido a razones prácticas.

\subsection{Método de resolución del problema y resultados}

Debido a las características del problema formulado, se pueden encontrar mejores valores para $T, M$ y $D$ resolviendo el POMO asociado. En esta aplicación, para resolver el problema se ha usado el AGMO basado en el SPEA-2 descrito en el apartado 4.4.2, considerando el vector decisión dado por (5-1), el vector objetivo dado por (5-2) y el vector de restricciones dado por (5-3), junto con las restricciones impuestas directamente a las variables y criterios de decisión (Tabla 5-3 y Tabla 5-4). La Tabla 5-4 muestra los resultados de la optimización multiobjetivo para una ejecución típica. Las columnas 1 a 3 muestran los valores para las variables de decisión $T, M$ y $D$. Las columnas 4 a 9 muestran los resultados obtenidos para cada uno de los seis criterios de aceptación considerados en el estudio. La primera fila presenta los valores iniciales. En la segunda fila se muestran los valores de restricción para los criterios de decisión. En las filas restantes de la Tabla 5-4 se presentan un conjunto de soluciones no dominadas del problema.

De este conjunto de soluciones se ha de adoptar una decisión al respecto de la solución finalmente a adoptar basada en la mejora del área objeto de nuestra atención. Así, si lo que interesa es la mejora de las especificaciones técnicas de funcionamiento (en los programas de RI-TS, RI-AOT, RI-STI) el criterio $\mathrm{RAMS}+\mathrm{C}$ más interesante a seguir debería ser el Coste, el riesgo simple y la contribución al riesgo anual.

La Figura 5-1 muestra gráficamente la distribución de las soluciones no dominadas encontradas por el AGMO en el espacio Costes - Riesgo simple Contribución al riesgo anual. Usando esta información no solamente es más 
fácil tomar una decisión final centrada en la mejora de las especificaciones técnicas sino que también asegura que los restantes atributos RAMS+C satisfacen los criterios de aceptación.

Tabla 5-4: Resultados de la optimización

\begin{tabular}{|c|c|c|c|c|c|c|c|c|}
\hline \multicolumn{3}{|c|}{$\begin{array}{l}\text { Vector Variables } \\
\text { Decisión (x) }\end{array}$} & \multicolumn{6}{|c|}{ Criterios de decisión } \\
\hline$T(h)$ & $M(h)$ & $D(h)$ & $\begin{array}{c}\text { No- } \\
\text { fiabilidad } \\
\left(u_{r}\right)(-)\end{array}$ & $\begin{array}{c}\text { Indisp. } \\
\text { detectada } \\
\left(\mathrm{u}_{\mathrm{tmc}}\right)(-)\end{array}$ & $\begin{array}{c}\text { Incremento } \\
\text { cond. del } \\
\text { riesgo } \\
\left(\Delta R_{E D G}\right) \\
(1 / a n ̃ o)\end{array}$ & $\begin{array}{l}\text { Riesgo } \\
\text { simple } \\
\left(\mathrm{r}_{\text {EDG }}\right)(-)\end{array}$ & $\begin{array}{l}\text { Contribución } \\
\text { riesgo anual } \\
\left(R_{\text {EDG }}\right)(1 / \text { año) }\end{array}$ & $\begin{array}{l}\text { Coste } \\
\text { (€/año) }\end{array}$ \\
\hline \multicolumn{9}{|c|}{ Valores Iniciales } \\
\hline 720 & 8760 & 72 & $2,76 \mathrm{E}-3$ & $9,49 \mathrm{E}-3$ & $5,13 E-5$ & $4,22 \mathrm{E}-7$ & $6,28 \mathrm{E}-7$ & $6,58 \mathrm{E} 4$ \\
\hline Restric & cciones & & $\leq 5,0 \mathrm{E}-2$ & $\leq 2,0 \mathrm{E}-2$ & $\leq 1,0 \mathrm{E}-3$ & $\leq 5,0 \mathrm{E}-7$ & $\leq 1,628 \mathrm{E}-6$ & $\leq 6,58 \mathrm{E} 4$ \\
\hline \multicolumn{9}{|c|}{ Conjunto Óptimo de Pareto } \\
\hline 720 & 24540 & 48 & $4,06 \mathrm{E}-3$ & $9,00 \mathrm{E}-3$ & $5,24 \mathrm{E}-5$ & 2,87E-7 & $6,84 \mathrm{E}-7$ & $6,24 \mathrm{E} 4$ \\
\hline 552 & 25260 & 74 & $3,28 \mathrm{E}-3$ & $1,16 \mathrm{E}-2$ & $5,15 E-5$ & $4,35 \mathrm{E}-7$ & $7,67 \mathrm{E}-7$ & $4,57 \mathrm{E} 4$ \\
\hline 576 & 43260 & 56 & $4,59 \mathrm{E}-3$ & $1,11 \mathrm{E}-2$ & $5,27 \mathrm{E}-5$ & $3,37 \mathrm{E}-7$ & $8,25 \mathrm{E}-7$ & $5,50 \mathrm{E} 4$ \\
\hline 456 & 41820 & 61 & $3,67 \mathrm{E}-3$ & $1,39 \mathrm{E}-2$ & 5,17E-5 & $3,60 \mathrm{E}-7$ & $9,09 \mathrm{E}-7$ & $5,72 \mathrm{E} 4$ \\
\hline 1200 & 8700 & 64 & $4,24 \mathrm{E}-3$ & $6,06 \mathrm{E}-3$ & $5,30 \mathrm{E}-5$ & $3,87 \mathrm{E}-7$ & $5,46 \mathrm{E}-7$ & $6,02 \mathrm{E} 4$ \\
\hline 744 & $4 \quad 41820$ & 51 & $5,65 \mathrm{E}-3$ & $8,66 \mathrm{E}-3$ & $5,38 \mathrm{E}-5$ & $3,13 \mathrm{E}-7$ & $7,70 \mathrm{E}-7$ & $5,45 \mathrm{E} 4$ \\
\hline 1824 & $4 \quad 33900$ & 40 & $1,14 \mathrm{E}-2$ & $3,76 \mathrm{E}-3$ & $6,00 \mathrm{E}-5$ & $2,74 \mathrm{E}-7$ & $9,11 \mathrm{E}-7$ & $5,44 \mathrm{E} 4$ \\
\hline 912 & 212300 & 51 & $3,72 \mathrm{E}-3$ & $7,45 \mathrm{E}-3$ & $5,23 E-5$ & $3,05 \mathrm{E}-7$ & $5,84 \mathrm{E}-7$ & $6,26 \mathrm{E} 4$ \\
\hline 744 & $4 \quad 38220$ & 61 & $5,34 \mathrm{E}-3$ & 8,67E-3 & 5,35E-5 & $3,73 \mathrm{E}-7$ & $7,50 \mathrm{E}-7$ & $4,24 \mathrm{E} 4$ \\
\hline 888 & 318780 & 75 & $4,30 \mathrm{E}-3$ & $7,46 \mathrm{E}-3$ & $5,28 \mathrm{E}-5$ & 4,52E-7 & $6,21 \mathrm{E}-7$ & 3,97E4 \\
\hline 4416 & 20940 & 46 & $2,03 \mathrm{E}-2$ & $1,87 \mathrm{E}-3$ & $7,00 \mathrm{E}-5$ & $3,68 \mathrm{E}-7$ & $1,56 \mathrm{E}-6$ & $3,89 \mathrm{E} 4$ \\
\hline 504 & $4 \quad 13740$ & 64 & $2,38 \mathrm{E}-3$ & $1,29 \mathrm{E}-2$ & $5,07 \mathrm{E}-5$ & $3,71 \mathrm{E}-7$ & $7,74 \mathrm{E}-7$ & $6,48 \mathrm{E} 4$ \\
\hline 360 & 31020 & 65 & $2,56 \mathrm{E}-3$ & $1,75 \mathrm{E}-2$ & $5,06 \mathrm{E}-5$ & $3,76 \mathrm{E}-7$ & $1,02 \mathrm{E}-6$ & $6,47 \mathrm{E} 4$ \\
\hline 384 & 20940 & 82 & $2,26 \mathrm{E}-3$ & $1,66 \mathrm{E}-2$ & $5,04 \mathrm{E}-5$ & $4,72 \mathrm{E}-7$ & $9,49 \mathrm{E}-7$ & $5,72 \mathrm{E} 4$ \\
\hline 1296 & 12300 & 71 & $5,07 \mathrm{E}-3$ & $5,45 \mathrm{E}-3$ & $5,38 \mathrm{E}-5$ & $4,36 \mathrm{E}-7$ & $5,66 \mathrm{E}-7$ & $4,51 \mathrm{E} 4$ \\
\hline 1104 & 38220 & 53 & 7,67E-3 & $5,96 \mathrm{E}-3$ & $5,60 \mathrm{E}-5$ & $3,39 \mathrm{E}-7$ & 7,63E-7 & $4,21 \mathrm{E} 4$ \\
\hline 1512 & 20220 & 48 & $7,19 \mathrm{E}-3$ & $4,56 \mathrm{E}-3$ & 5,59E-5 & 3,07E-7 & $6,58 \mathrm{E}-7$ & 4,64E4 \\
\hline 1440 & 23820 & 65 & $7,47 \mathrm{E}-3$ & $4,73 \mathrm{E}-3$ & 5,61E-5 & 4,17E-7 & $6,85 \mathrm{E}-7$ & $3,28 \mathrm{E} 4$ \\
\hline 1728 & 15900 & 37 & $7,29 \mathrm{E}-3$ & $4,12 \mathrm{E}-3$ & $5,62 \mathrm{E}-5$ & 2,37E-7 & $6,42 \mathrm{E}-7$ & $6,48 \mathrm{E} 4$ \\
\hline 1368 & 29580 & 39 & $8,02 E-3$ & $4,90 \mathrm{E}-3$ & $5,66 \mathrm{E}-5$ & $2,52 \mathrm{E}-7$ & 7,31E-7 & $6,21 \mathrm{E} 4$ \\
\hline
\end{tabular}




\begin{tabular}{|c|c|c|c|c|c|c|c|c|}
\hline $\mathrm{T}$ (h) & M (h) & $\mathrm{D}(\mathrm{h})$ & $\begin{array}{c}\text { No- } \\
\text { fiabilidad } \\
\left(u_{r}\right)(-)\end{array}$ & $\begin{array}{l}\text { Indisp. } \\
\text { detectada } \\
\left(\mathrm{u}_{\mathrm{tmc}}\right)(-)\end{array}$ & $\begin{array}{c}\text { Incremento } \\
\text { cond. del } \\
\text { riesgo } \\
\left(\Delta R_{E D G}\right) \\
(1 / a n ̃ o)\end{array}$ & $\begin{array}{l}\text { Riesgo } \\
\text { simple } \\
\left(\mathrm{r}_{\mathrm{EDG}}\right)(-)\end{array}$ & $\begin{array}{l}\text { Contribución } \\
\text { riesgo anual } \\
\left(R_{\text {EDG }}\right)(1 / a n ̃ o)\end{array}$ & $\begin{array}{c}\text { Coste } \\
\text { (€/año) }\end{array}$ \\
\hline 2208 & 35340 & 38 & $1,41 \mathrm{E}-2$ & $3,16 \mathrm{E}-3$ & $6,27 \mathrm{E}-5$ & $2,72 \mathrm{E}-7$ & $1,08 \mathrm{E}-6$ & $5,63 \mathrm{E} 4$ \\
\hline 2064 & 8700 & 73 & $6,91 \mathrm{E}-3$ & $3,91 \mathrm{E}-3$ & $5,61 \mathrm{E}-5$ & $4,68 \mathrm{E}-7$ & 6,07E-7 & $5,26 \mathrm{E} 4$ \\
\hline 480 & 40380 & 68 & $3,75 \mathrm{E}-3$ & $1,32 \mathrm{E}-2$ & $5,18 \mathrm{E}-5$ & $4,02 \mathrm{E}-7$ & $8,80 \mathrm{E}-7$ & $4,91 \mathrm{E} 4$ \\
\hline 648 & 37500 & 77 & 4,67E-3 & $9,91 \mathrm{E}-3$ & $5,28 E-5$ & 4,64E-7 & $7,70 \mathrm{E}-7$ & $3,70 \mathrm{E} 4$ \\
\hline
\end{tabular}

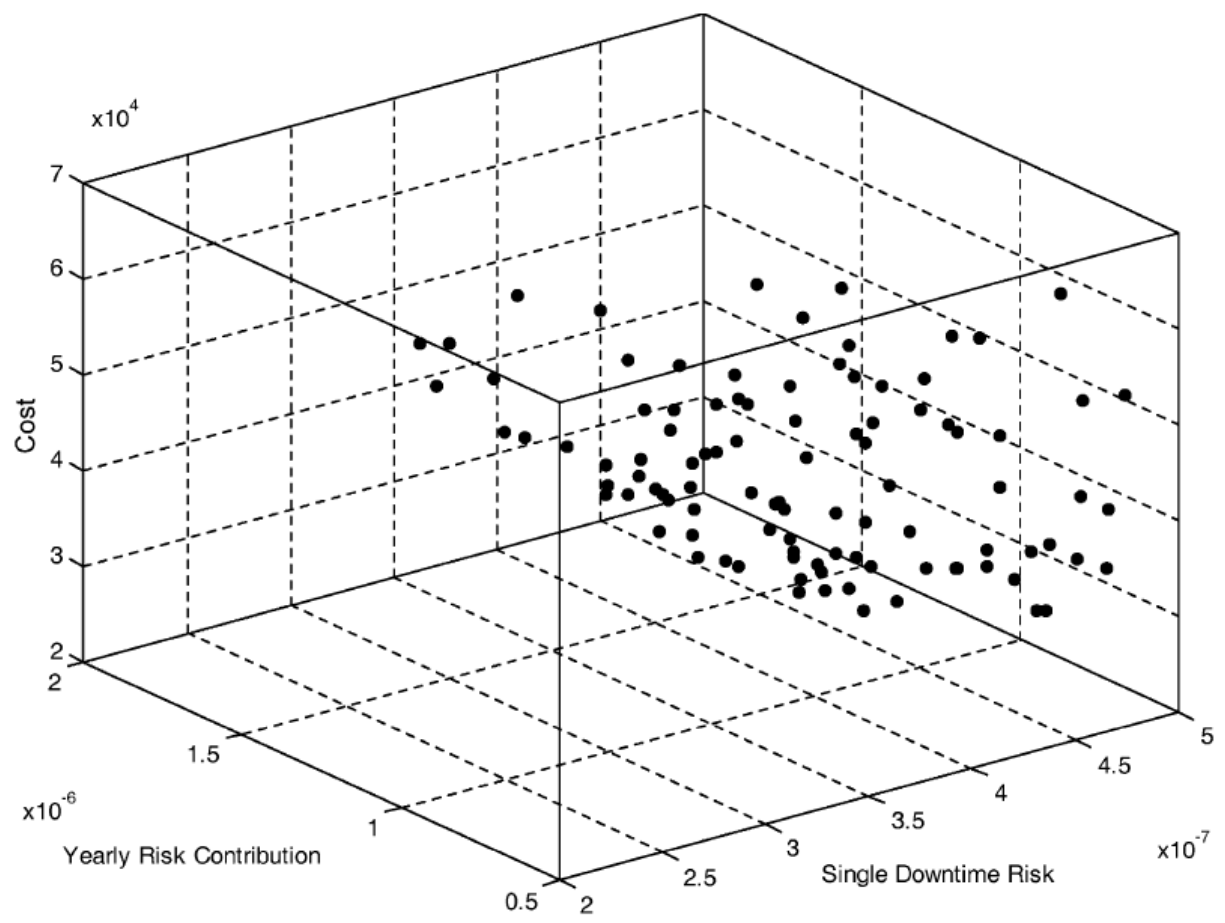

Figura 5-1: Soluciones no dominadas: coste - riesgo simple - contribución al riesgo anual

Por otro lado, si el principal objetivo es mejorar el mantenimiento (como sucede en los programas MRI, RCM) se presenta la Figura 5-2 de posibles alternativas en el espacio Coste-No fiabilidad- Indisponibilidad detectada. 
También en este caso los restantes criterios RAMS $+C$ se mantienen bajo valores aceptables ya que la optimización se ha llevado a cabo teniendo en cuanta todos ellos, por lo que todo el conjunto de soluciones satisfará los requerimientos iniciales del problema.

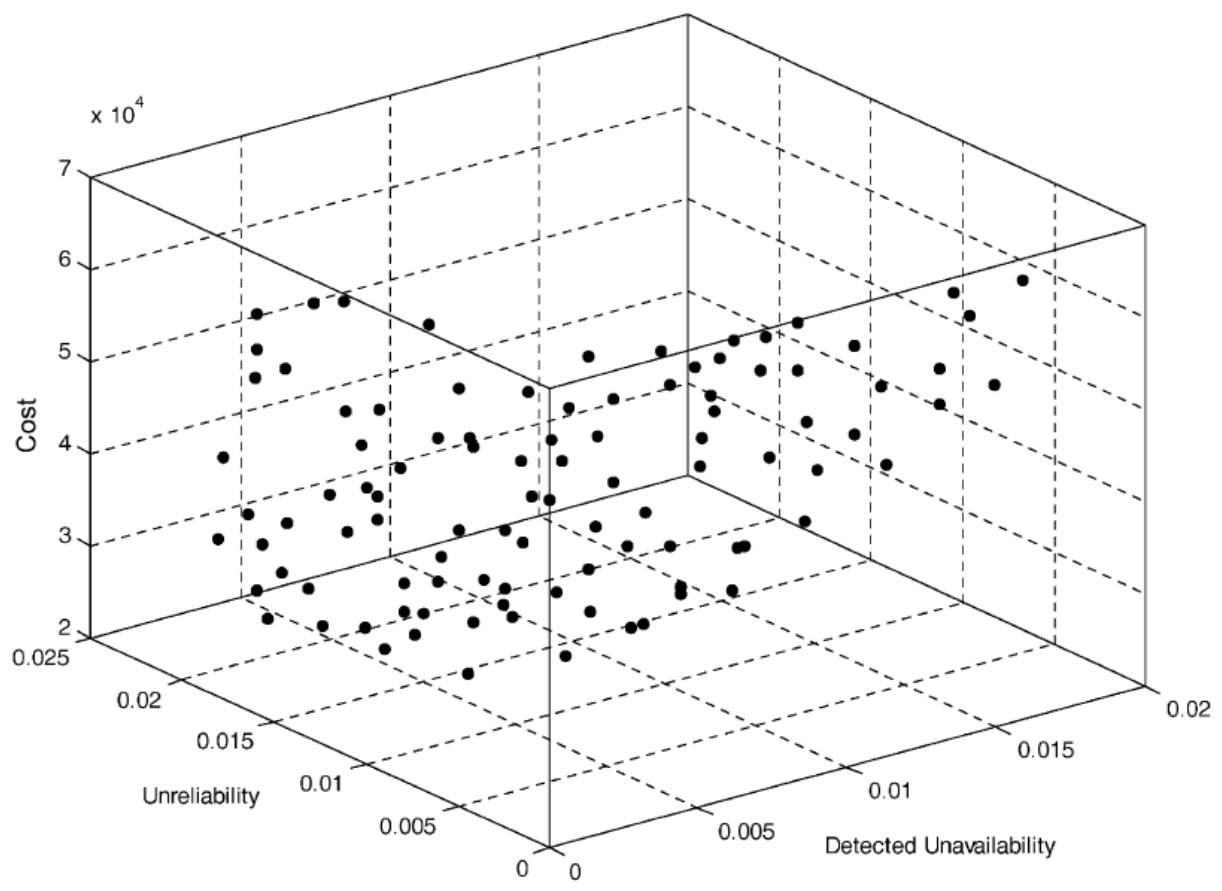

Figura 5-2: Soluciones no dominadas: coste - no fiabilidad - indisponibilidad detectada.

\subsection{Discusión}

Con este caso de aplicación, la toma de decisiones multicriterio en cambios de vigilancia y mantenimiento se ha formulado como un problema de optimización multiobjetivo basado en criterios $\mathrm{RAMS}+\mathrm{C}$, usando vectores objetivo, decisión y restricciones como se ha propuesto. Las soluciones a este problema se han obtenido resolviendo dicho problema de optimización multiobjetivo tomando como funciones objetivo todos los criterios RAMS $+C$. La solución final se puede tomar dependiendo del área principal de mejora, 
pero esta solución asegura que todos los criterios RAMS+C se cumplen ya que la optimización se ha realizado considerando todo el conjunto de restricciones en los criterios de aceptación de forma simultánea.

Con este ejemplo de aplicación se ha introducido la necesidad de considerar una aproximación más amplia a la toma de decisiones multiobjetivo en los cambios de requisitos de las actividades de vigilancia y mantenimiento bajo criterios RAMS $+C$. Esto conduce a una toma de decisiones más consistente con el papel de las especificaciones técnicas de funcionamiento y el mantenimiento, y el objetivo de los actuales programas de mejora de las centrales nucleares (RI-LB, RCM, LMP LRA y MRI, comentados en el apartado 2.5).

La toma de decisiones multiobjetivo se ha fundamentado en tres pilares. Se han considerado, de forma simultánea, todos los parámetros relativos a vigilancia y mantenimiento para la toma de decisiones. Se han considerado los criterios RAMS $+\mathrm{C}$ de forma simultánea, pero se pueden incorporar otros criterios adicionales. Los objetivos numéricos son necesarios para comparar los criterios frente a niveles de aceptación, y dichos objetivos se encuentran claramente definidos actualmente en las centrales nucleares.

El caso de aplicación para el sistema de generadores diesel de emergencia justifica la importancia de la aproximación propuesta y al mismo tiempo demuestra la viabilidad de la metodología para resolver esta clase de problemas. Así, los resultados permiten obtener un conjunto de valores para los parámetros relativos a la vigilancia y mantenimiento basados en conseguir valores óptimos en los atributos RAMS $+C$. Sin embargo, también se evidencian efectos contrarios de tales variables en los criterios de decisión, lo que nos hace pensar en la posibilidad de necesitar llegar a un equilibrio para conseguir el mejor compromiso entre los diferentes criterios. 



\section{Optimización multiobjetivo de requisitos de vigilancia a nivel de sistema considerando dependencia temporal}

\subsection{Descripción del problema}

Los requisitos de vigilancia son parte de las especificaciones técnicas de las centrales nucleares, la cuales establecen los intervalos entre pruebas, $T$, y a menudo la estrategia de pruebas. El objetivo principal es asegurar que el equipo de seguridad, normalmente en espera, esté disponible en el momento que sea necesario durante un accidente.

Se requieren pruebas de vigilancia que se ejecuten de forma periódica (semanal, mensual, cuatrimestral, etc.) según el intervalo entre pruebas, $T$, establecido. Estos mismos intervalos entre pruebas son los mismos para los equipos redundantes, de diferentes trenes del mismo sistema de seguridad.

Para llevar a cabo estas pruebas se distinguen dos tipos de estrategias de pruebas: secuenciales y escalonadas. En las estrategias secuenciales las pruebas de $n$ equipos redundantes se ejecutan consecutivamente a intervalos fijos, $T$, uno detrás del otro. En la estrategia escalonada, la prueba de cada uno de los equipos se lleva a cabo en intervalos fijos, pero de tal forma que el 
intervalo entre cada una de las pruebas de los $n$ equipos redundantes es $T / n$. De esta forma se reduce el tiempo en que cada uno de los equipos redundantes es probado, con la consiguiente ventaja, en especial, en la detección de fallos debidos a causas comunes a todos los equipos.

Esta relación entre la estrategia de pruebas y el intervalo entre las mismas afecta a la cuantificación de la indisponibilidad del sistema y por tanto al llamado riesgo limitado por la prueba.

La prueba sobre componentes en espera, detecta los fallos y permite mantener bajo control el riesgo de la planta. Sin embargo, un excesivo número de pruebas, puede afectar negativamente a la seguridad de la planta y además puede imponer costes y cargas innecesarias para los recursos humanos de la planta (Samanta 1995). Además, una estrategia de pruebas inapropiada puede dar lugar a inoperabilidades simultáneas de equipos importantes, que pueden aumentar la vulnerabilidad de la planta como consecuencia de la configuración de riesgo de la misma.

Por lo general, tanto el intervalo entre pruebas como la estrategias de pruebas se suelen establecer de forma determinista. Sin embargo, hay un consenso sobre la idea que ambos tienen una importancia potencial sobre la reducción de riesgos y costes de la central (Cepin 2002).

Muchos de los trabajos desarrollados se han basado en la optimización de estos requisitos de vigilancia bajo criterios $R A M S+C$, pero centrándose bien en la optimización del intervalo entre pruebas, o bien en la estrategia de las mismas (Cepin 2002), (Martorell 2002), (Martorell 2004), (Marseguerra 2000), (Busacca 2001) y (Lapa 2003).

En este caso de aplicación se presenta el desarrollo y aplicación de una aproximación para optimizar conjuntamente el intervalo entre pruebas y la estrategia de las mismas considerando el efecto temporal de la estrategia de pruebas en los equipos de seguridad que normalmente se encuentran en espera en las centrales nucleares.

\subsection{Formulación del problema}

En el presente problema de optimización multiobjetivo, POMO, de los requisitos de vigilancia se adoptan como variables de decisión el intervalo entre pruebas y los parámetros asociados a la estrategia de las pruebas bajo criterios de indisponibilidad y costes, $\mathrm{A}[\mathrm{U}]+\mathrm{C}$, como caso particular de la toma de decisiones bajo criterios RAMS $+C$. 
La búsqueda de soluciones a este POMO requiere de una adecuada modelización para simular cómo los cambios en las variables afectadas modifican al coste y a la disponibilidad. La idea de esta aproximación es ajustar tales variables para llegar al mejor compromiso entre dichos criterios que entran en conflicto. El problema se complica como consecuencia de las restricciones que se imponen en la búsqueda de soluciones, como pueden ser los valores requeridos para indisponibilidad y riesgo y los rangos permitidos para las variables.

Para la simulación de cómo los cambios simultáneos de las variables que definen los intervalos entre pruebas, y la estrategia de las mismas pueden afectar a la indisponibilidad y al coste, se han de usar modelos probabilísticos dependientes del tiempo para cuantificar las contribuciones de la fiabilidad, disponibilidad y costes de los equipos que consideren explícitamente el efecto del intervalo entre pruebas y la estrategia de las pruebas. Los modelos utilizados en esta aplicación son los expuestos en el apartado 3.7.

El sistema seleccionado para realizar la optimización es el sistema de inyección a alta presión (HPIS) de una central nuclear de agua a presión. En la Figura 6-1 se muestra una versión simplificada del mismo adaptada de la literatura (Harunuzzaman 1996). Este sistema se encuentra normalmente en espera y consiste en tres bombas y siete válvulas organizadas como se muestra en la Figura 6-1. En condiciones accidentales, el HPIS se puede usar para extraer el calor del reactor en aquellos sucesos en los cuales los generadores de vapor no se encuentren disponibles. Por ejemplo, en el caso de un accidente de pérdida de inventario por una rotura pequeña, la función de seguridad del HPIS es extraer agua desde el tanque almacenamiento de agua de recarga e inyectarla en las ramas frías del sistema de refrigeración del reactor a través de cualquiera de los dos caminos de inyección ( $A \circ B$ ). Normalmente, las bombas descargan en los caminos de inyección A y B a través de las válvulas 3 y 5 , aunque también las válvulas de interconexión 4 , 6 y 7 proporcionan un camino alternativo en caso de fallar la alimentación normal.

De acuerdo con los requisitos típicos de bombas y válvulas dentro de las especificaciones técnicas del HPIS, el tiempo entre pruebas es $T=2184 \mathrm{~h}$. Además se ha adoptado los datos más relevantes de indisponibilidad y costes para este caso de aplicación desde (Martorell 2002).

La Tabla 6-1 muestra valores típicos de los datos de fiabilidad, pruebas, mantenimientos para bombas y válvulas del sistema de inyección a alta presión, los cuales son necesarios para cuantificar la indisponibilidad del sistema y el coste anual del mismo según los modelos expuestos en las 
ecuaciones de los apartados 3.1, 3.4 y en especial del apartado 3.7 donde se consideran la incorporación de la dependencia temporal.

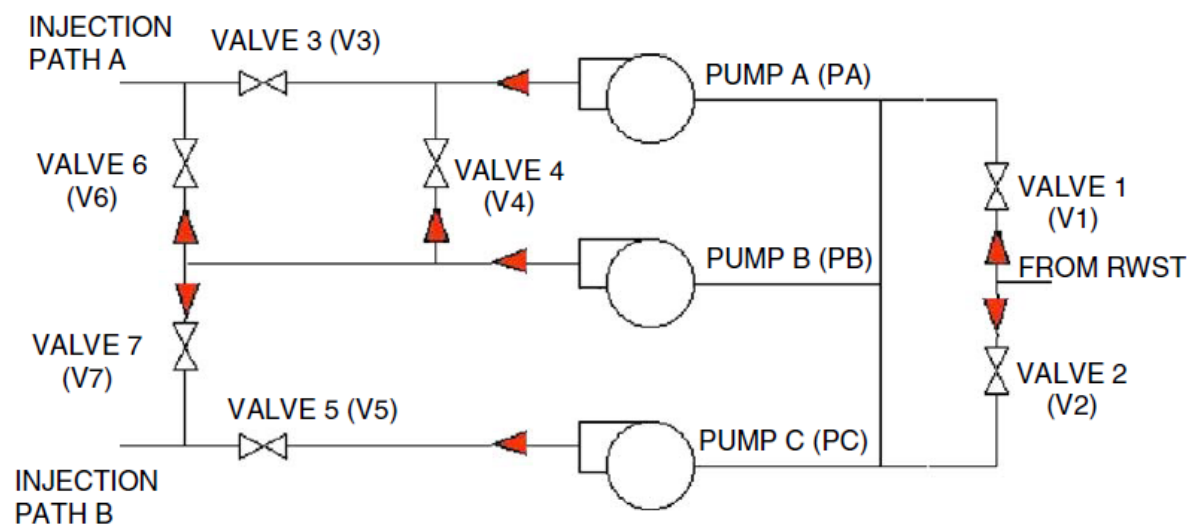

Figura 6-1: Sistema de inyección a alta presión.

Tabla 6-1: Datos típicos para bombas y válvulas, e intervalos actuales de pruebas, mantenimientos y reemplazamientos

Descripción

Fallo

Tasa de fallo a la espera

Probabilidad de fallo por demanda

\section{Degradación}

Tasa de envejecimiento lineal

\section{Pruebas}

Intervalos entre pruebas

Tiempo medio ejecución prueba

\section{Mantenimiento}

Tiempo medio fuera de servicio por reparación

Tiempo medio ejecución mantenimiento preventivo

Intervalos mantenimiento preventivo $\alpha\left(h^{-2}\right)$

2,20E-11 4,57E-10

Parámetro Bombas Válvulas

$\begin{array}{ccc}\lambda_{0}\left(\mathrm{~h}^{-1}\right) & 3,89 \mathrm{E}-6 & 5,83 \mathrm{E}-6 \\ \rho(-) & 5,30 \mathrm{E}-4 & 1,82 \mathrm{E}-3\end{array}$

$\begin{array}{ccc}\mathrm{T}(\mathrm{h}) & 2184 & 2184 \\ \mathrm{~T}(\mathrm{~h}) & 4 & 1\end{array}$

$\mu(h) \quad 24 \quad 2,6$

$\sigma(\mathrm{h}) \quad 10 \quad 1$

$M(h) \quad 4320 \quad 4320$




\begin{tabular}{lccc}
\hline Descripción & Parámetro & Bombas & Válvulas \\
\hline Reemplazamiento & & & \\
Tiempo medio para reemplazamiento & $\Gamma(\mathrm{h})$ & 50 & 6 \\
Periodo tras el cual se hace reemplazamiento & $\mathrm{RP}(\mathrm{h})$ & 350400 & 87600 \\
\hline
\end{tabular}

La Tabla 6-2 muestra valores para la cuantificación de costes de los modelos dados por las ecuaciones del apartado 3.6 del capítulo 3 .

Tabla 6-2: Datos típicos de costes para válvulas y bombas

\begin{tabular}{|c|c|c|c|}
\hline Descripción & Parámetro & Bombas & Válvulas \\
\hline \multicolumn{4}{|l|}{ Costes } \\
\hline Contribución coste anual de pruebas & $c_{t}(€)$ & 800 & 200 \\
\hline $\begin{array}{l}\text { Contribución coste anual de mantenimientos } \\
\text { preventivos }\end{array}$ & $\mathrm{c}_{\mathrm{m}}(€)$ & 8000 & 800 \\
\hline $\begin{array}{l}\text { Contribución coste anual de mantenimiento } \\
\text { correctivo }\end{array}$ & $\mathrm{c}_{\mathrm{c}}(€)$ & 28800 & 3120 \\
\hline $\begin{array}{l}\text { Contribución coste anual } \\
\text { reemplazamiento }\end{array}$ & $\mathrm{c}_{\mathrm{o}}(€)$ & 36000 & 3000 \\
\hline
\end{tabular}

La Tabla 6-3 muestra una posible distribución del intervalo entre pruebas, $T$, agrupados en tres grupos, de acuerdo a la $T$ aplicable $\{T 1, T 2, T 3\}$ y de la estrategia de las mismas, definido a partir del tiempo de la primera prueba para cada uno de los componentes del sistema de inyección de agua a presión, TP, agrupados en 6 grupos $\{T A, T B, T C, T D, T E, T F\}$.

De acuerdo con estrategia actual para el sistema de inyección de agua a presión, también se pueden aplicar las siguientes relaciones en relación al periodo entre pruebas, $T$ :

$\mathrm{T} 1=2184 \mathrm{~h} ; \quad \mathrm{T} 2=\mathrm{k} 2 \cdot \mathrm{T} 1 ; \quad \mathrm{T} 3=\mathrm{k} 3 \cdot \mathrm{T} 1 ;$ 
con $k 2$ y $k 3$ la relación de proporcionalidad de los intervalos entre pruebas de los grupos 2 y 3 con el intervalo entre pruebas para el grupo 1 .

Tabla 6-3: Grupos de estrategias agrupados en función del tiempo de la primera prueba (TP) e intervalos entre pruebas (T) del sistema de inyección a alta presión.

\begin{tabular}{l|llllllllll}
\hline T vs TP & PA & PB & PC & V1 & V2 & V3 & V4 & V5 & v6 & V7 \\
\hline T1 & TA & TB & TC & TA & TC & TA & & TC & & \\
T2 & & & & & & & TD & & & \\
T3 & & & & & & & & & TE & TF \\
\hline
\end{tabular}

Se puede establecer también para el tiempo de realización de la primera prueba, TP:
$\mathrm{TA}=24 \mathrm{~h}$;
$\mathrm{TC}=72 \mathrm{~h} ;$
$\mathrm{TE}=\mathrm{TB}+\mathrm{ke} \cdot \mathrm{T} 1$;
$\mathrm{TB}=48 \mathrm{~h}$;
$\mathrm{TD}=\mathrm{TB}+\mathrm{kdT} 1$
$\mathrm{TF}=\mathrm{TB}+\mathrm{kf} \cdot \mathrm{T} 1$

donde $k d$, ke y $k f$ son constantes que permiten relacionar la estrategia de pruebas de los grupos $\mathrm{D}, \mathrm{E}$ y $\mathrm{F}$ con el intervalo entre pruebas del grupo 1.

A partir de la Tabla 6-3 y de las relaciones anteriores, se deduce que el proceso de toma de decisiones se puede basar en el siguiente vector de variables de decisión:

$\mathrm{x}=\{\mathrm{T} 1, \mathrm{k} 2, \mathrm{k} 3, \mathrm{TA}, \mathrm{TB}, \mathrm{TC}, \mathrm{kd}, \mathrm{ke}, \mathrm{kf}\}$

En el presente caso de aplicación, el vector de decisión se puede simplificar usando los valores típicos $\{k 2=3 ; k 3=3 ; k d=0 ; k e=1 ; k f=2\}$ de acuerdo a las prácticas corrientes en las centrales nucleares, lo que conduce al siguiente vector de decisión:

$\mathrm{x}=\{\mathrm{T} 1, \mathrm{TA}, \mathrm{TB}, \mathrm{TC}\}$ 
Además se adopta como vector de criterios de decisión,

$y=f(x)=\left(U_{m}(x), U_{t m}(x), C(x)\right)$

siendo $U_{m}(x)$ la indisponibilidad media del sistema de inyección a alta presión, $U_{t m}(x)$ la máxima indisponibilidad dependiente del tiempo de dicho sistema, y el $C(x)$ el coste medio anual para un $x$ dado, tal y como se definieron en el apartado 3.7.

Es conveniente fijar un punto de partida para comparar los resultados después del proceso de optimización. Las condiciones iniciales se representan por la particularización del vector de decisiones (6-2) para el conjunto inicial de tiempos entre pruebas y estrategias como sigue:

$\mathrm{x}=\{2184,24,48,72\}$

Utilizando los modelos del apartado 3.7 para $U_{m}(x), U_{t m}(x)$ y $C(x)$, el vector objetivo inicial queda como:

$\mathrm{y}_{\mathrm{i}}=\left\{\mathrm{U}_{\mathrm{m}}\left(\mathrm{x}_{\mathrm{i}}\right), \mathrm{U}_{\mathrm{tm}}\left(\mathrm{x}_{\mathrm{i}}\right), \mathrm{C}\left(\mathrm{x}_{\mathrm{i}}\right)\right\}=\{5,22688 \mathrm{E}-4 ; 5,62661 \mathrm{E}-2 ; 19752,3\}$

La Figura 6-2 representa la evolución de la indisponibilidad con dependencia temporal del sistema de inyección a alta presión para el caso inicial.

El vector de criterios de decisión (6-3) actúa como vector de funciones objetivo para el POMO sujeto a un número de restricciones dado por:

$\mathrm{g}(\mathrm{x})=\left\{\mathrm{U}_{\mathrm{m}}(\mathrm{x}) \leq 2,0 \mathrm{E}-4, \mathrm{U}_{\mathrm{tm}}(\mathrm{x}) \leq 3,0 \mathrm{E}-2, \mathrm{C}(\mathrm{x}) \leq 5,0 \mathrm{E} 4\right\}$

Además, también se imponen restricciones en el rango de variabilidad de valores permitidos para las variables de decisión. Así el rango de valores permitidos para la programación de pruebas, tiempo hasta la primera prueba, $T P$, es variable ya que en general es dependiente del intervalo entre pruebas, $T$. Por lo tanto, las restricciones particulares impuestas al rango de valores permitidos dependerá del problema en particular (caso) a ser resuelto, como se discute a continuación. Se han propuesto 4 casos en función del objetivo perseguido en cada uno de ellos. 


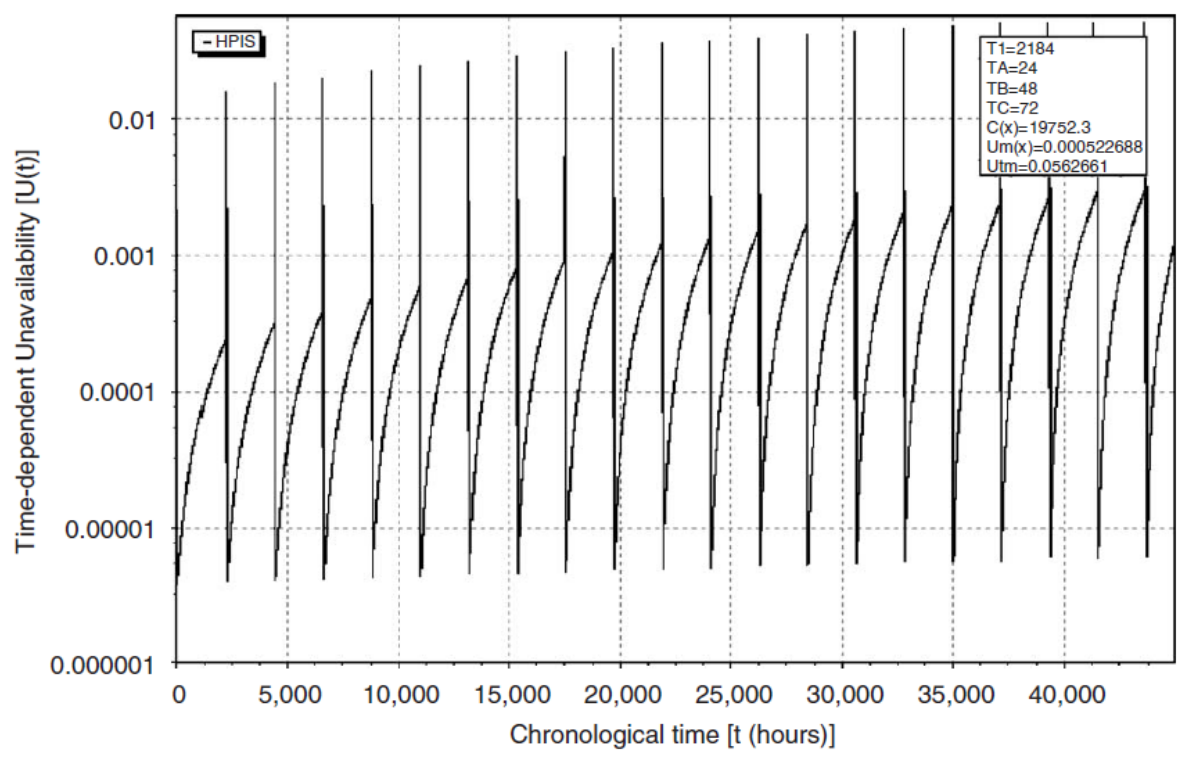

Figura 6-2: Indisponibilidad dependiente del tiempo. Caso Inicial

\subsection{Método de resolución y resultados}

La Tabla 6-4 resume los detalles de los cuatro casos ejecutados y el quinto con el que se ha comparado y que se han considerado en este ejemplo de aplicación, esto es, la formulación del problema, función objetivo y restricciones, vector decisión, resolución del problema, técnica usada, mecanismo de codificación, operadores genéticos y parámetros.

El objetivo detrás de los casos seleccionados es demostrar en una aproximación paso a paso los principios subyacentes del algoritmo propuesto y las ventajas y desventajas de usar este método. Así, la ejecución de los casos 1-3 tiene por objetivo obtener el conjunto de soluciones de Pareto para las situaciones extremas para observar la importancia y el papel que tanto el intervalo entre pruebas $T$ y la estrategia de pruebas, definida por TP, tienen en el proceso de optimización. Por otro lado, la ejecución del caso 4 tiene por objetivo derivar el conjunto de Pareto usando la búsqueda directa.

El caso 1 centra su atención en la optimización del intervalo entre pruebas, manteniendo constante la estrategia de pruebas, mientras que el caso 2 y 3 optimizan esta estrategia para unos tiempos entre pruebas determinados. El 
caso 4 busca optimizar tanto el intervalo entre pruebas como la estrategia de pruebas de una forma conjunta.

La Figura 6-3 muestra un gráfico 3D con cinco superficies que resumen los resultados del conjunto de análisis. La Figura 6-4 muestra los mismos resultados en un gráfico $2 \mathrm{D}$ con comentarios destacando los puntos más relevantes encontrados, que se encuentran resumidos en la Tabla 6-5. Todos estos resultados representan una ejecución particular para cada caso.

En dicha tabla se presentan posibles soluciones obtenidas de las diferentes fronteras de Pareto que minimizan la indisponibilidad media $\left(\operatorname{Min}_{m}(x)\right)$, o el coste anual $(\operatorname{Min} C(x))$.

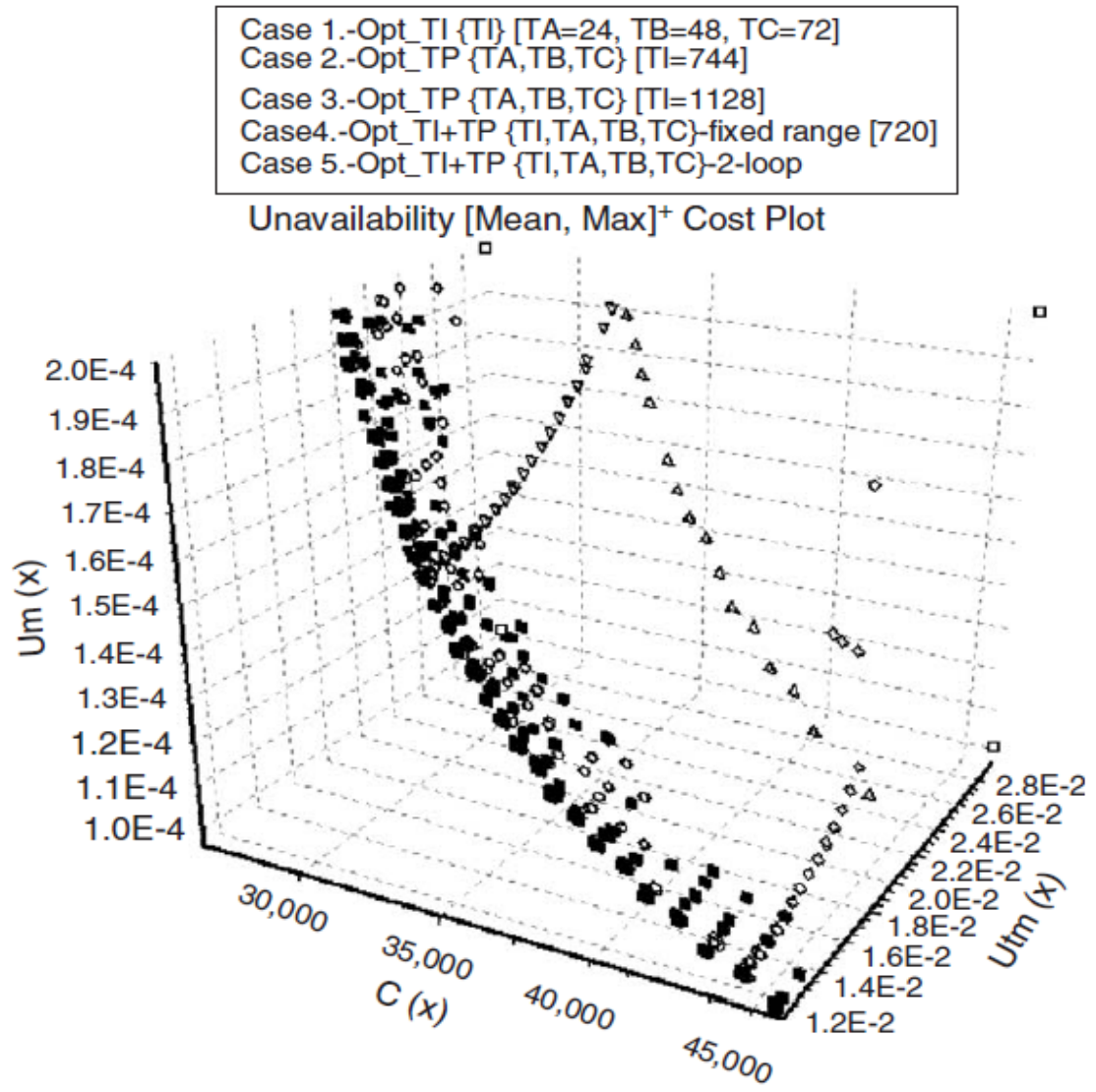

Figura 6-3: Resumen de resultados $U m(x)$. Utm(x) y $C(x)^{1}$

${ }^{1}$ El caso 5 corresponde a un estudio paralelo incluido en (Martorell 2006) 
Optimización multiobjetivo de requisitos de vigilancia a nivel de sistema considerando dependencia temporal

Tabla 6-4: Descripción de los casos de ejecución

\begin{tabular}{|c|c|c|c|c|}
\hline Ítem & Caso 1 & Caso 2 & Caso 3 & Caso 4 \\
\hline \multicolumn{5}{|l|}{ Formulación } \\
\hline Vector decisión & $\{\mathrm{T} 1\}$ & $\{\mathrm{TA}, \mathrm{TB}, \mathrm{TC}\}$ & $\{\mathrm{TA}, \mathrm{TB}, \mathrm{TC}\}$ & $\{\mathrm{T} 1, \mathrm{TA}, \mathrm{TB}, \mathrm{TC}\}$ \\
\hline Vector objetivo & & \multicolumn{3}{|c|}{$\mathrm{U}_{\mathrm{m}}(\mathrm{x}), \mathrm{U}_{\mathrm{tm}}(\mathrm{x}), \mathrm{C}(\mathrm{x})$} \\
\hline $\begin{array}{l}\text { Vector } \\
\text { restricciones }\end{array}$ & & \multicolumn{3}{|c|}{$\mathrm{U}_{\mathrm{m}}(\mathrm{x}) \leq 2,0 \mathrm{E}-4, \mathrm{U}_{\mathrm{tm}}(\mathrm{x}) \leq 3,0 \mathrm{E}-2, \mathrm{C}(\mathrm{x}) \leq 5,0 \mathrm{E} 4$} \\
\hline \multicolumn{5}{|c|}{ Rango y paso [min, max, paso] } \\
\hline $\mathrm{T} 1$ & {$[720,8760,24]$} & $\mathrm{T} 1=744$ & $T 1=1128$ & {$[720,8760,24]$} \\
\hline TA & $\mathrm{TA}=24$ & {$[0,744,24]$} & {$[0,1128,24]$} & {$[0,720,24]$} \\
\hline TB & $\mathrm{TB}=48$ & {$[0,744,24]$} & {$[0,1128,24]$} & {$[0,720,24]$} \\
\hline TC & $\mathrm{TC}=72$ & {$[0,744,24]$} & {$[0,1128,24]$} & {$[0,720,24]$} \\
\hline \multicolumn{5}{|l|}{ Resolución } \\
\hline Algoritmo & $\begin{array}{l}\text { AGMO base } \\
\text { SPEA2 }\end{array}$ & $\begin{array}{l}\text { AGMO base } \\
\text { SPEA2 }\end{array}$ & $\begin{array}{l}\text { AGMO base } \\
\text { SPEA2 }\end{array}$ & $\begin{array}{l}\text { AGMO base } \\
\text { SPEA2 }\end{array}$ \\
\hline Codificación AG & Entero $\{\mathrm{T} 1\}$ & $\begin{array}{c}\text { Entero } \\
\{\mathrm{TA}, \mathrm{TB}, \mathrm{TC}\}\end{array}$ & $\begin{array}{c}\text { Entero } \\
\{\mathrm{TA}, \mathrm{TB}, \mathrm{TC}\}\end{array}$ & $\begin{array}{c}\text { Entero } \\
\{\mathrm{T} 1, \mathrm{TA}, \mathrm{TB}, \mathrm{TC}\}\}\end{array}$ \\
\hline \multicolumn{5}{|l|}{ Parámetros AG } \\
\hline Generaciones & 3000 & 3000 & 3000 & 3000 \\
\hline Tamaño pob. & 100 & 100 & 100 & 100 \\
\hline Inicialización & Aleatoria & Aleatoria & Aleatoria & Aleatoria \\
\hline Selec. de pareja & Por torneo & Por torneo & Por torneo & Por torneo \\
\hline Op. de cruce & 1 pto de cruce & 1 pto de cruce & 1 pto de cruce & 1 pto de cruce \\
\hline Tasa de cruce & 0,9 & 0,9 & 0,9 & 0,9 \\
\hline Op. mutación & Mutación Flip & Mutación Flip & Mutación Flip & Mutación Flip \\
\hline Tasa de mutación & 0,3 & 0,3 & 0,3 & 0,3 \\
\hline \% Reemplazo & 1 & 1 & 1 & 1 \\
\hline Evaluación & Domi. Pareto & Domi. Pareto & Domi. Pareto & Domi. Pareto \\
\hline $\begin{array}{l}\text { Manejo } \\
\text { Restricciones }\end{array}$ & $\begin{array}{l}\text { Penalización } \\
\text { dinámica y } \\
\text { rechazo }\end{array}$ & $\begin{array}{l}\text { Penalización } \\
\text { dinámica y } \\
\text { rechazo }\end{array}$ & $\begin{array}{l}\text { Penalización } \\
\text { dinámica y } \\
\text { rechazo }\end{array}$ & $\begin{array}{l}\text { Penalización } \\
\text { dinámica y } \\
\text { rechazo }\end{array}$ \\
\hline Escala ajuste & Lineal & Lineal & Lineal & Lineal \\
\hline Terminación & Generaciones & Generaciones & Generaciones & Generaciones \\
\hline Tamaño Ext. & 100 & 100 & 100 & 100 \\
\hline
\end{tabular}




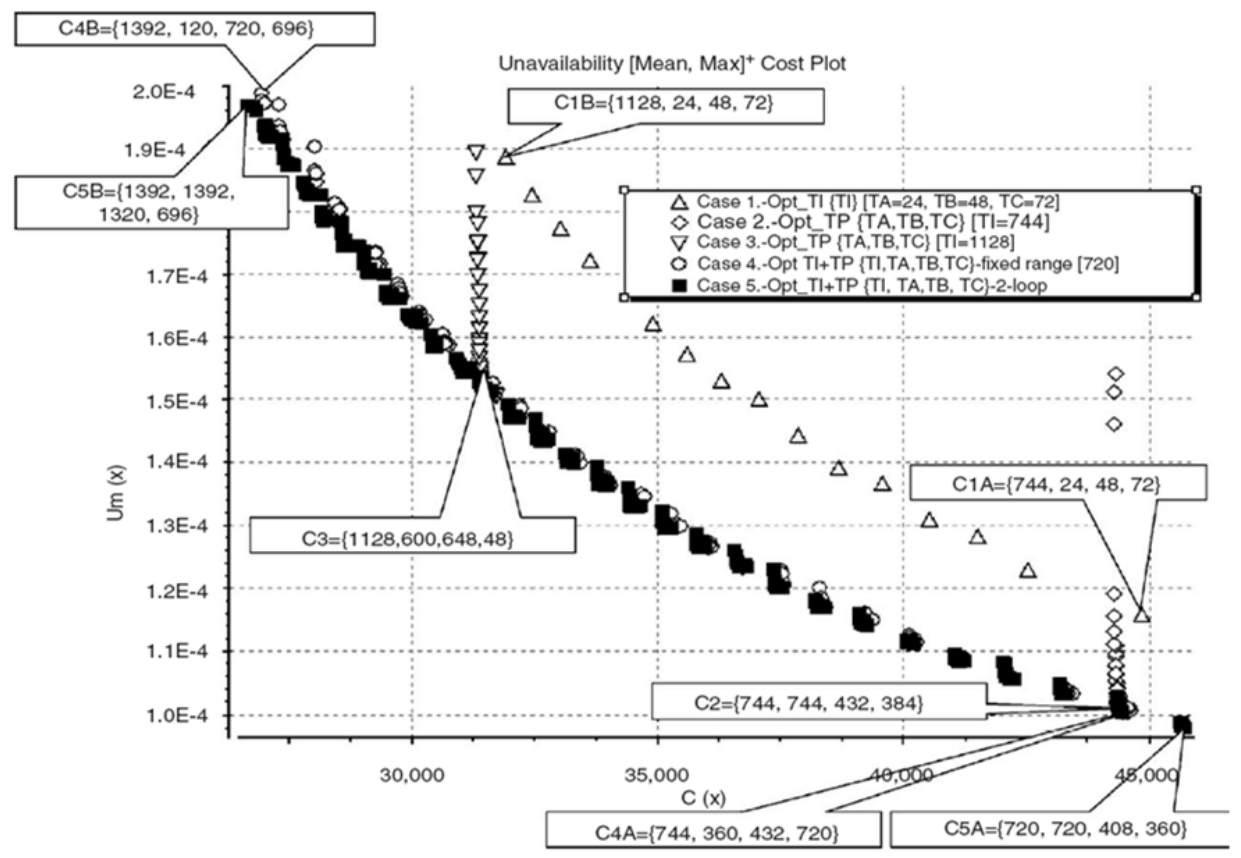

Figura 6-4: Resumen de resultados $\operatorname{Utm}(x)$ y $C(x)^{2}$

En el caso 1, se obtiene la superficie que representa el conjunto de soluciones de Pareto al POMO donde solamente T1, es decir, el intervalo entre pruebas, T, actúa como variable de decisión mientras que TA, TB y TC, es decir la estrategia de las pruebas, representado por los tiempos hasta la primera prueba, TP, permanecen constantes en sus valores iniciales. Los puntos relevantes C1A y C1B, ver Tabla 6-5 y Figura 6-4, representan soluciones individuales que pertenecen al conjunto de Pareto, las cuales minimizan $U_{m}(x)$ y $C(x)$, respectivamente.

En el caso 2, se obtiene la superficie que representa el conjunto de soluciones de Pareto para el POMO donde solamente TA, TB y TC, es decir la estrategia de las pruebas, actúan como variables de decisión mientras que $\mathrm{T} 1$, es decir el intervalo entre pruebas, permanece constante. La búsqueda de la solución al POMO parte desde el punto $\{744,24,48,72\}$, que corresponde al valor T1 de la solución con el valor más bajo, medio y máximo de indisponibilidad del sistema después de resolver el POMO anterior del

\footnotetext{
${ }^{2}$ El caso 5 corresponde a un estudio paralelo incluido en (Martorell 2006)
} 
caso 1 que considera solamente el T1 como variable de decisión (esto es, el punto $\mathrm{C} 1 \mathrm{~A}$ que es solución para el caso 1). Para este caso el punto relevante C2, ver Tabla 6-5 y Figura 6-4, representa una solución individual perteneciente a este conjunto de Pareto, que minimiza $C(x)$.

Tabla 6-5: Soluciones relevantes para el intervalo entre pruebas y estrategia de las pruebas bajo varios casos

\begin{tabular}{|c|c|c|c|c|c|c|}
\hline \multirow[b]{2}{*}{ Punto } & \multicolumn{2}{|c|}{ Caso 1} & \multirow{3}{*}{$\begin{array}{c}\text { Caso } 2 \\
\mathrm{C} 2 \\
\operatorname{MinC}(\mathbf{x})\end{array}$} & \multirow{3}{*}{$\begin{array}{c}\text { Caso } 3 \\
\text { C3 } \\
\text { MinC(x) }\end{array}$} & \multicolumn{2}{|c|}{ Caso 4} \\
\hline & C1A & C1B & & & C4A & C4B \\
\hline Solución & $\operatorname{MinU}_{m}(\mathbf{x})$ & $\operatorname{Min} C(x)$ & & & $\operatorname{MinU}_{m}(x)$ & $\operatorname{MinC}(x)$ \\
\hline \multicolumn{7}{|c|}{ Variable Decisión (x) } \\
\hline $\mathrm{T} 1$ & 744 & 1128 & 744 & 1128 & 744 & 1392 \\
\hline TA & 24 & 24 & 744 & 600 & 360 & 120 \\
\hline TB & 48 & 48 & 432 & 648 & 432 & 720 \\
\hline $\mathrm{TC}$ & 72 & 72 & 384 & 48 & 720 & 696 \\
\hline \multicolumn{7}{|c|}{ Criterio de decisión } \\
\hline $\mathrm{U}_{\mathrm{m}}(\mathrm{x})$ & $1,16 \mathrm{E}-4$ & $1,89 \mathrm{E}-4$ & $1,01 \mathrm{E}-4$ & $1,51 \mathrm{E}-4$ & $1,01 \mathrm{E}-4$ & $1,99 \mathrm{E}-4$ \\
\hline $\mathrm{U}_{\mathrm{tm}}(\mathrm{x})$ & $1,99 \mathrm{E}-2$ & 2,95E-2 & $1,18 \mathrm{E}-2$ & 1,67E-2 & $1,18 \mathrm{E}-2$ & 2,31E-2 \\
\hline$C(x)$ [€/año] & 44854 & 31912 & 44438 & 31716 & 44451 & 26947 \\
\hline Tiempo CPL & & 0,86 & 0,86 & 0,86 & & \\
\hline
\end{tabular}

El caso 3 proporciona la superficie que representa el conjunto de soluciones de Pareto para el POMO donde solamente TA, TB y TC, es decir la estrategia de las pruebas, actúan como variables de decisión mientras que T1, es decir el intervalo entre pruebas, permanece constante. Ahora, la búsqueda de soluciones del POMO parte del punto $\{1128,24,48,72\}$ que corresponde con el valor T1 con el Coste más bajo después de resolver el POMO del caso 1 que consideraba solamente T1 como variable de decisión (esto es, punto C1B para el caso 1). Para este caso, el punto relevante C3, ver Tabla 6-5 y Figura 6-4, representa una solución individual perteneciente a este conjunto de Pareto que minimiza $C(x)$.

El análisis del caso 4 lleva a la superficie que se obtiene de la optimización donde todo el conjunto de variables de decisión $\{T 1, T A, T B, T C\}$ participan 
en la búsqueda directa de soluciones, participando tanto el intervalo entre pruebas como la estrategia de las mismas en el POMO planteado. En el caso 4 , el conjunto de valores permitidos para el TA, TB y TC, se encuentra dentro del rango $[0,720]$. Para este caso el punto relevante $C 4 A$ y C4B representan soluciones individuales pertenecientes a este conjunto de Pareto que minimizan $U_{m}(x)$ y $C(x)$, respectivamente, ver Tabla 6-5 y Figura 6-4.

Comparando los resultados obtenidos para el caso 1, donde solamente el intervalo entre pruebas se consideraba como variable de decisión en el POMO, y el caso 4, se puede concluir que la optimización de $T$ conlleva un ajuste aproximado de los Requisitos de Vigilancia que conduce a muy buenas puntuaciones de $U_{m}(x)$ (punto $C 1 A$ ) y $C(x)$ (punto $C 1 B$ ). Una vez hecho esto, es posible un ajuste fino de los requisitos de vigilancia mediante la optimización de las estrategias de pruebas, como se observa al comparar los resultados del caso 2 y 3 , después de resolver el POMO manteniendo ahora constante el intervalo entre pruebas y tomando como variables de decisión las estrategias TA, TB y TC.

Después de este estudio, se puede concluir que es necesaria la optimización simultánea del intervalo entre pruebas y la estrategia de las mismas (ajuste aproximado y fino) debido a dos razones principalmente:

- $\quad$ El ajuste fino representa el efecto bien conocido donde una prueba escalonada (una mayor o menor distribución uniforme de la estrategia de pruebas a lo largo del intervalo entre las mismas) conduce a mejores puntuaciones en minimizar la media y el máximo punto estimado de la indisponibilidad del sistema (Samanta 1995). De este modo, es posible evolucionar, Figura 6-4, desde la superficie aproximada (caso 1) hasta la superficie base (caso 4) manteniendo constante el intervalo entre pruebas (por ejemplo 744 o 1128) y optimizando el resto de variables de decisión (caso 2 y 3 ).

- $\quad$ Sin embargo, por ejemplo, un valor de T1=1392 es una solución no permitida para el POMO que considera solamente el intervalo entre pruebas como una variable de decisión manteniendo la estrategia de las pruebas en sus valores iniciales (caso 1), ya que violará las restricciones impuestas a la indisponibilidad media y máxima. Por lo tanto, siguiendo los principios anteriores, se puede esperar que el ajuste fino de los parámetros TP simultáneamente con los ajustes aproximados de los parámetros $T$ permitirá reducir el valor para la indisponibilidad media y máxima, de esta forma, permitiendo la oportunidad de sobrepasar 1128 para $T$. 


\subsection{Discusión}

Como conclusiones de los resultados presentados se puede decir, en general, que se ha estudiado el problema de optimización simultánea de requisitos de vigilancia de equipos de seguridad basado en criterios de fiabilidad, disponibilidad, mantenibilidad, seguridad y coste.

La viabilidad de la metodología, basada en el uso de modelos probabilistas con dependencia temporal, permite simular cómo cambios en el intervalo entre pruebas, $T$, y de estrategia de pruebas, TP, afectan a los atributos de disponibilidad y costes. Los modelos han sido usados para evaluar las funciones objetivo del problema de optimización multiobjetivo siendo resueltos en los casos de aplicación mediante una aproximación AGMO basado en SPEA-2 que ejecuta la búsqueda directa de la solución en todo el espectro de POMO.

Comenzando desde los resultados obtenidos para los casos de aplicación enfocado a la optimización del intervalo entre pruebas y la estrategia de las mismas para el sistema de inyección de agua a presión de una central nuclear basado en criterios de disponibilidad y de coste se obtiene las siguientes conclusiones:

- La optimización del intervalo entre pruebas, $T$ (T1 para el caso de aplicación), permite un ajuste aproximado de los requisitos de vigilancia en las especificaciones técnicas

- La optimización de la estrategia de pruebas, TP (TA, TB, y TC para el caso de aplicación), permite un ajuste fino de los requisitos de vigilancia en las especificaciones técnicas siguiendo, el principio que sugiere la prueba escalonada de los trenes redundantes.

- El ajuste fino podría seguir a uno aproximado en un camino iterativo conduciendo a buenos resultados. Esta idea da crédito a anteriores trabajos donde $T$ y $T P$ son optimizados independientemente uno de otro.

- El ejemplo de aplicación muestra que los mejores resultados se obtienen cuando se considera la optimización simultánea de $T$ y $T P$ como una consecuencia de las restricciones impuestas en las variables de decisión y funciones objetivo.

Se puede observar que el caso de aplicación al HPIS es un problema pequeño (con cuatro variables de decisión) que es fácil de resolver mediante 
una exhaustiva enumeración de las variables. Sin embargo, se debe destacar que una evaluación del vector objetivo usando los modelos probabilistas con dependencia temporal necesitan de aproximadamente $1 \mathrm{~s}$ de tiempo de ejecución. 



\section{Optimización multiobjetivo de la vigilancia y mantenimiento a nivel de sistema considerando el efecto de las incertidumbres}

\subsection{Optimización considerando incertidumbres en las variables de decisión}

\subsubsection{Descripción del problema}

En los estudios sobre mejoras de las especificaciones técnicas de funcionamiento de las centrales nucleares existen pocos estudios que introduzcan el tratamiento de las incertidumbres. Sin embargo, la experiencia operacional de planta muestra que las pruebas incluidas en las especificaciones técnicas no se realizan, por lo general, en un intervalo constante. Por el contrario, el intervalo entre dos pruebas consecutivas varía dentro de un intervalo definido por un valor medio y una cierta tolerancia permitida. Esto es debido a las diferentes situaciones en que puede presentarse la operación de una planta, como un alto riesgo de pérdida de potencia externa, recargas, indisponibilidad de recurso, restricciones de los reguladores, etc. Es por ello más realista considerar este intervalo entre las pruebas como una variable aleatoria más que un valor constante (Lapa 
2003).

Esta aplicación se centra en la optimización del tiempo entre pruebas basado en criterios de indisponibilidad y costes, considerando dichos intervalos como variables de decisión no constantes, sino que varían dentro de un rango de tolerancia.

\subsubsection{Formulación del problema.}

El siguiente caso de aplicación se centra en el problema de la optimización de las actividades de pruebas del sistema simplificado de inyección de alta presión (HPIS) de un reactor de agua a presión propuesto en el capítulo 6 (ver Figura 6-1). Este problema considera el efecto de la incertidumbre asociado al intervalo del tiempo entre pruebas en la optimización multiobjetivo considerando los criterios de indisponibilidad y coste. Para ello se ha usado un procedimiento de simulación por Monte Carlo y se ha utilizado las ventajas de los estadísticos de orden para proporcionar intervalos de tolerancia de distribución libre para la estimación de la indisponibilidad y costes basados en un número mínimo de ejecuciones necesarias $(N=459)$ para garantizar una probabilidad de contención o cobertura $(\gamma=0.99)$ con un nivel de confianza $(\beta=0.99)$. Para su resolución se ha utilizado los métodos planteados en el apartado 4.5.2.

La Tabla 6-1 y Tabla 6-2 (apartado 6.2) muestran valores típicos de los datos de fiabilidad, pruebas, mantenimientos y costes individuales para bombas y válvulas del HPIS, necesarios para cuantificar la indisponibilidad del sistema y el coste anual del mismo.

De acuerdo a las especificaciones técnicas del sistema de inyección a alta presión, se realizan tres tipos de pruebas periódicas sobre los distintos equipos del sistema. La Tabla 7-1 muestra unos posibles intervalos entre pruebas para las bombas y válvulas del sistema agrupadas en tres grupos distintos $\{T 1, T 2, T 3\}$ asumibles de implantar en la central: $T 1$ para las válvulas 1, 2, 3 y 5 en la aspiración y descarga de las bombas y también para las bombas A, B y C, T2 para la válvula 4 de interconexión y T3, para las válvulas 6 y 7 de interconexión (ver Figura 6-1). 
Tabla 7-1: Intervalo entre pruebas para el Sistema de inyección a alta presión.

\begin{tabular}{l|llllllllll}
\hline $\begin{array}{l}\text { Tiempo entre } \\
\text { pruebas }\end{array}$ & PA & PB & PC & V1 & V2 & V3 & V4 & V5 & V6 & V7 \\
\hline T1 & X & X & X & X & X & X & & X & & \\
T2 & & & & & & & X & & & \\
T3 & & & & & & & & & X & X \\
\hline
\end{tabular}

donde, de acuerdo con la actual política de mantenimiento para un HPIS típico de una central nuclear, se aplica la siguiente relación $T 2=k 2^{*} T 1$ y $T 3=k 3^{*} T 1$, donde $k 2$ y $k 3$ determinan la relación de proporcionalidad de los intervalos entre pruebas de los grupos 2 y 3 con el intervalo entre pruebas para el grupo 1.

La Tabla 7-2 presenta dichas variables, sus valores iniciales y el rango de variación permitido.

Tabla 7-2: Condiciones iniciales y rango de valores permitidos para las variables de decisión.

\begin{tabular}{llccc}
\hline $\begin{array}{l}\text { Vector de } \\
\text { decisión }\end{array}$ & Rango & \multicolumn{3}{c}{ Valor inicial $\mathrm{x}_{\mathrm{i}}$} \\
\cline { 2 - 4 } & Min & Max & $\Delta$ & \\
\hline T1 (h) & 24 & 8760 & 24 & 270 \\
k2=T2/T1 & 1 & 10 & 1 & 1 \\
k3=T3/T1 & 1 & 10 & 1 & 1 \\
\hline
\end{tabular}

Para obtener la función de indisponibilidad del sistema, se desarrolla el árbol de fallos del sistema y se cuantifica usando la aproximación de conjuntos mínimos de corte (Minimal Cut Set, MCS) y las ecuaciones (3-12), (3-13), (3-14) y (3-28). En lo relacionado la función de costes, el modelo correspondiente ha obtenido añadiendo los costes asociados con pruebas, mantenimiento preventivo, reparaciones y reemplazamientos a partir de las ecuaciones (3-17) a (3-22). 
Ambas funciones, indisponibilidad y coste, son a menudo consideradas como deterministas, en el sentido que cuando se especifica todas las variables de entrada necesarias para el modelo, solo proporciona un único valor como variable de salida. Sin embargo cuando se consideran incertidumbres en un parámetro del modelo, se asume que este valor puede fluctuar dependiendo de la función de distribución asociada.

En otros estudios, una vez que el algoritmo genético ha seleccionado el valor del intervalo entre pruebas, este permanece constante en el cálculo de la indisponibilidad y coste. Es más, la solución óptima se alcanza para un valor específico y no se consideran otras soluciones cercanas a este valor.

En planta, es difícil ejecutar la prueba en intervalos exactos. De hecho hay una cierta tolerancia alrededor del valor aceptado para ejecutar la prueba. De esta forma, es interesante evaluar si, este intervalo de tolerancia aceptado, afectará o no al coste e indisponibilidad del sistema.

Para esta aplicación, se asume que las pruebas ejecutadas con periodicidad T1, T2 y T3 presentan este tipo de incertidumbre en cuanto a su ejecución exacta.

En una primera aproximación, de acuerdo con el principio de la razón insuficiente (también conocido como principio de indiferencia, como se ve en (Helton 2004) ), se usa una distribución uniforme para caracterizar la incertidumbre en ausencia de información para diferenciar la credibilidad de diferentes alternativas, y también es apropiado si uno cree ciertamente que cada uno de los valores del intervalo es igualmente probable. Así en este caso se asume que los intervalos entre prueba T1, T2 y T3 son variables aleatorias con una distribución uniforme alrededor del valor esperado.

Asumir otro tipo de distribuciones podría afectar a la cuantificación de la incertidumbre. Para analizar el efecto de la distribución aleatoria en el cálculo de la incertidumbre se han estudiado dos distribuciones típicas más: una distribución normal y una lognormal.

\subsubsection{Método de resolución y resultados}

Se ha usado un AGMO adaptado para implementar la aproximación basada en el método de intervalos de tolerancia que permite manejar las incertidumbres en la optimización basada en criterios de indisponibilidad y coste de pruebas y mantenimientos (apartado 4.5.2). La Tabla 7-3 muestra los valores adoptados para los parámetros más relevantes del AGMO. En 
aras de la simplicidad, en este caso de aplicación $U(x)$ y $C(x)$ se asumen como independientes para seleccionar el tamaño de muestra adecuado a unos límites dados de $\gamma / \beta$, tal y como se describió en dicho apartado.

Como se ha mencionado con anterioridad, los primeros cálculos se han llevado a cabo asumiendo una distribución uniforme alrededor del valor esperado. Así, se ha considerado una desviación máxima respecto al valor esperado de 3,7 y 30 días respectivamente. La Figura 7-1 muestra la Frontera de Pareto obtenida para estos cálculos.

Tabla 7-3: Parámetros adoptados para el AGMO ajustado al método de intervalos de tolerancia.

\begin{tabular}{ll}
\hline Parámetro & Valor \\
\hline Generaciones (G) & 200 \\
Número de pruebas (N) & 459 (para $/ / \beta=0,99 / 0,99)$ \\
Tamaño Población (pS) & 100 \\
Inicialización & Aleatoria con inserción de valores iniciales \\
Tipo de Selección & Por torneo \\
Operador de cruces & Cruce por un punto \\
Tasa de cruces & 0,9 \\
Operador de Mutación & Mutación Gaussiana Real \\
Tasa de Mutación & 0,2 \\
Probabilidad de & 0,95 \\
Reemplazamiento (pR) & Dominancia de Pareto basado en SPEA2 \\
Evaluación & Penalización dinámica \\
Manejo de Restricciones & Reparto \\
Ajuste & Número de Generaciones \\
Terminación & 100 \\
Tamaño Externo &
\end{tabular}




\section{U-C Plot}

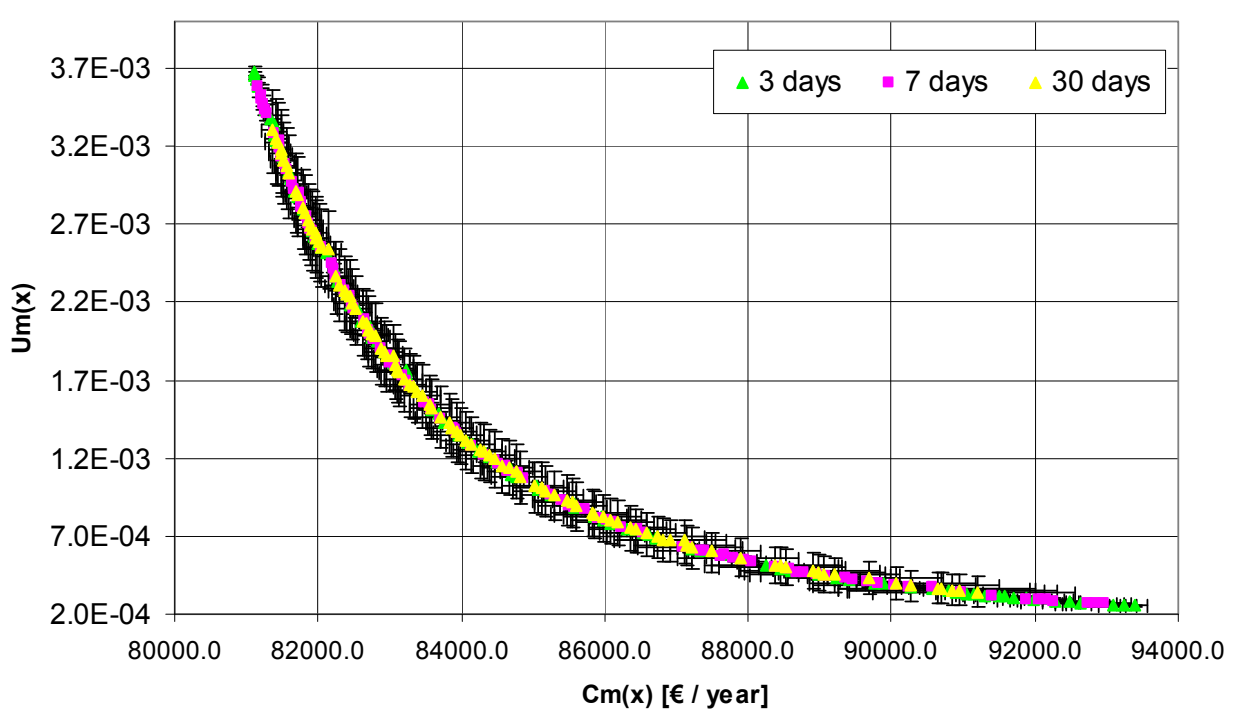

Figura 7-1: Resultados obtenidos considerando distribución uniforme

El intervalo de incertidumbre en los cálculos es apreciable cuando se considera una desviación de 30 días respecto al valor esperado. Mientras que en los cálculos en los que se supone una desviación de 3 y 7 días el intervalo de incertidumbre obtenido para los criterios de indisponibilidad y coste no es significativo.

Del análisis de los datos de pruebas y mantenimientos del sistema de extracción de calor residual de una planta nuclear comercial, se ha observado que el intervalo entre pruebas puede ajustarse a una distribución normal. Obteniendo que, la máxima desviación respecto al valor esperado, corresponde a dos veces la desviación estándar.

Se han ejecutado tres optimizaciones más asumiendo una distribución normal alrededor del valor esperado con tres desviaciones estándar diferentes de 1 , 2.3 y 10 días respectivamente. La Figura 7-2 muestra los resultados obtenidos para estas ejecuciones. Una vez más, se aprecia que para valores bajos de desviación en el tiempo entre pruebas, el intervalo de incertidumbre es poco apreciable. De la Figura 7-1 y Figura 7-2 se puede observar que el intervalo de incertidumbre es ligeramente inferior en los intervalos entre 
pruebas que siguen una distribución normal.

\section{U-C Plot}

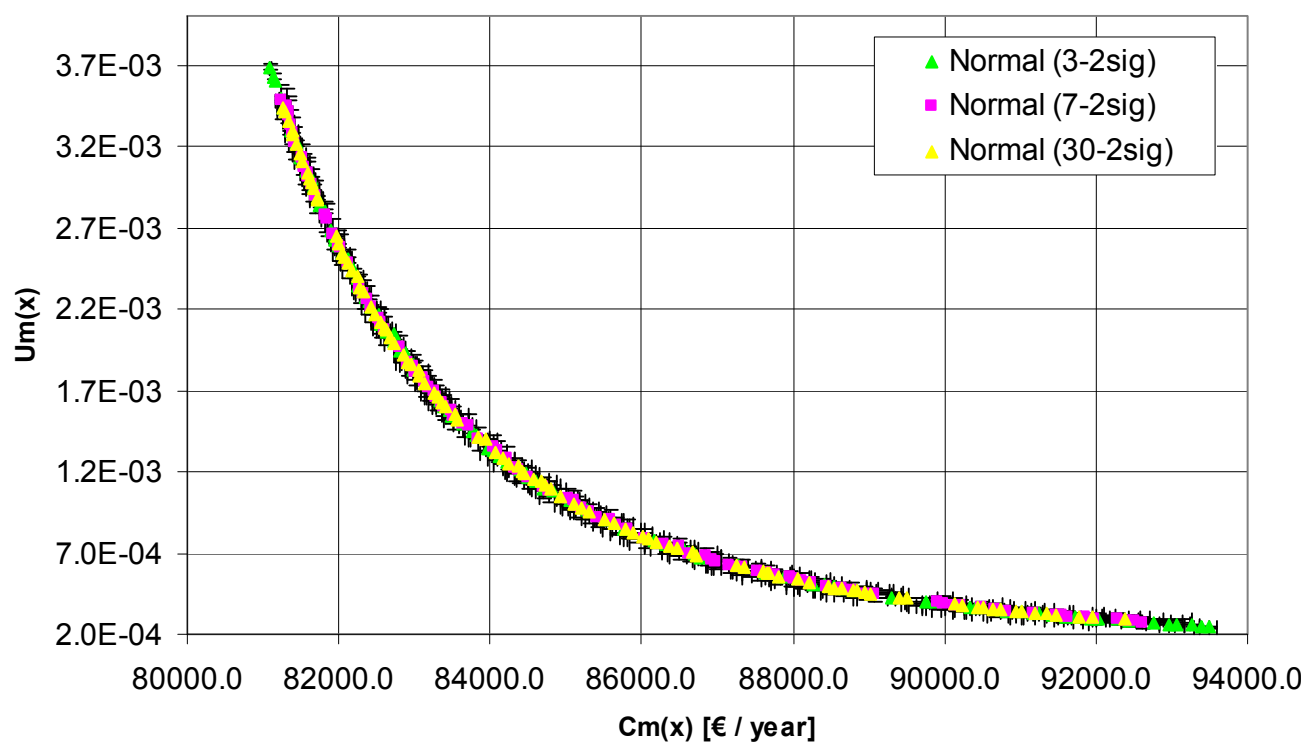

Figura 7-2: Resultados obtenidos considerando una distribución normal.

Se ha llevado a cabo una aplicación final considerando que el intervalo entre pruebas sigue una distribución lognormal, asumiendo que hay una gran probabilidad de retraso en la ejecución de la prueba debido al plan de pruebas y mantenimiento. La Figura 7-3, muestra los resultados obtenidos considerando que la variable de decisión en el problema de optimización sigue una distribución lognormal, con una desviación máxima respecto al valor esperado de 3 y 30 días. 


\section{U-C Plot}

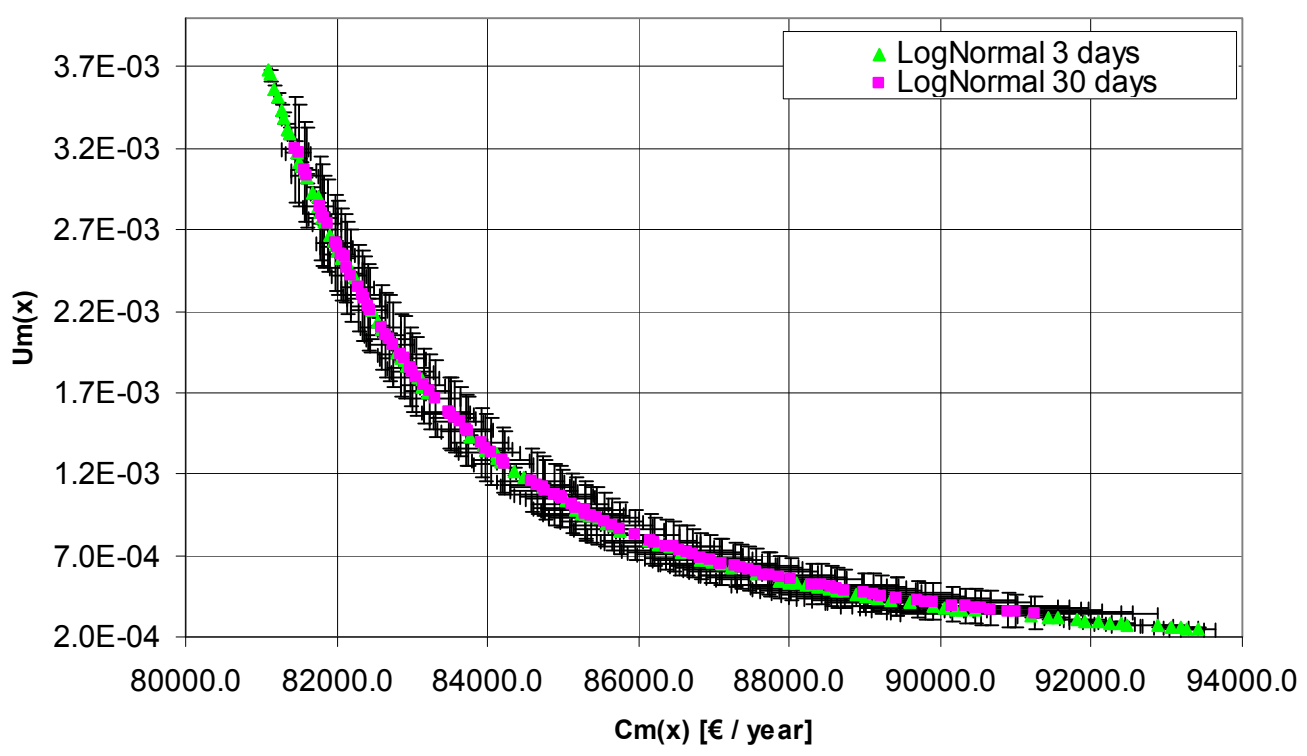

Figura 7-3: Resultados obtenidos considerando una distribución lognormal

En esta figura se puede observar que los intervalos de tolerancia obtenidos para los criterios de coste e indisponibilidad son mayores que los obtenidos con anterioridad.

Por otro lado de la comparación de la Figura 7-1, Figura 7-2 y Figura 7-3 se puede observar que la frontera final óptima de Pareto es igual para las tres distribuciones consideradas. Esto sugiere que el proceso de optimización encuentra el mismo conjunto de soluciones no dominadas para los criterios de coste e indisponibilidad sin dependencia del modelo de distribución seleccionado para la variable de decisión.

Para analizar si todas las optimizaciones ejecutadas encuentran el mismo espacio de variables de decisión, o si hay diferentes espacios de variables de decisión que proporcionan la misma solución, se ha llevado a cabo un análisis estadístico de las soluciones que pertenecen a las diferentes fronteras optimas de Pareto. Como ejemplo, la Figura 7-4 presenta el gráfico Box and Whisker para T1, considerando una desviación máxima de 30 días respecto al valor esperado para las tres distribuciones consideradas en los 
cálculos. En esta figura se observa que las medidas de la tendencia central, las medidas de variabilidad, y simetría son bastante similares, para todas las distribuciones analizadas.

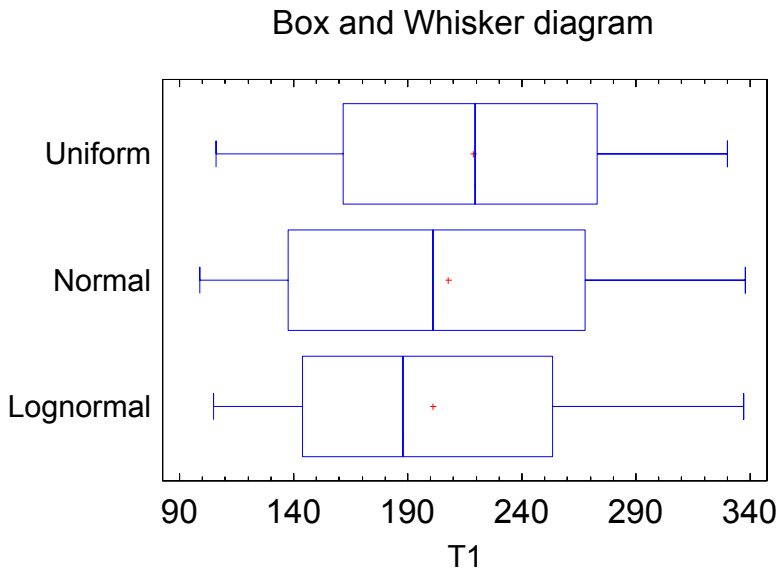

Figura 7-4: Gráfico Box and Whisker para T1 dependiendo del modelo de distribución.

Para analizar el efecto de la máxima tolerancia considerada, se ha realizado un análisis de los valores alcanzados por la frontera óptima de Pareto asumiendo una distribución normal. La Figura 7-5 muestra los valores obtenidos para T1. Una vez más se puede observar que las medidas de tendencia central, las medidas de variabilidad, y simetría son bastante similares, para los tres intervalos de tolerancia representados. El análisis también se ha ejecutado para $k 2$ y $k 3$ obteniéndose los mismos resultados.

Como resultado de este análisis se concluye que no hay diferencias significativas entre los valores alanzados por la variable de decisión en las diferentes optimizaciones. 
Box and Whisker diagram

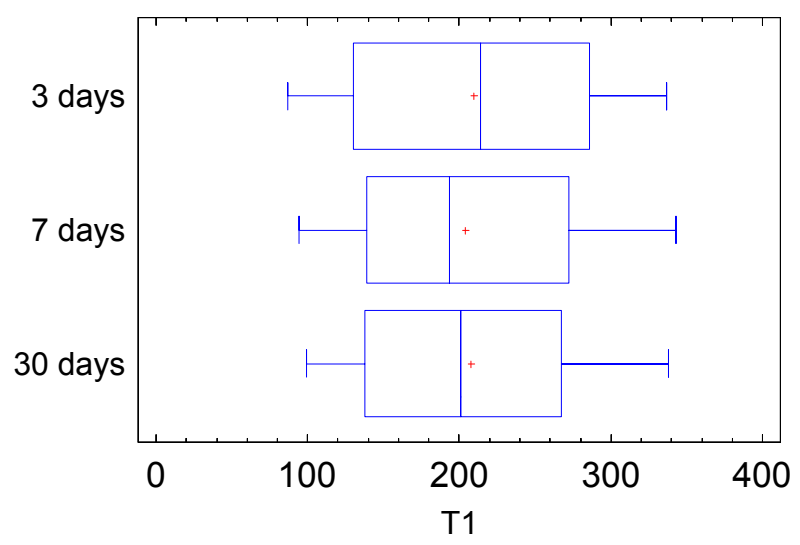

Figura 7-5: Gráfico Box and Whisker para T1 dependiente de la máxima tolerancia admitida.

\subsubsection{Discusión}

La experiencia operacional de las plantas nucleares muestra que el intervalo entre pruebas es fijado como un valor constante. En realidad, éstos varían dentro de un intervalo definido por el valor medio y una cierta tolerancia permitida. Es por ello que es más realista considerar dicho tiempo como una variable aleatoria que considerarlo constante como se ha hecho tradicionalmente.

Esta aplicación ha presentado la optimización del intervalo entre pruebas de un sistema de seguridad bajo criterios de indisponibilidad y coste, considerando que los tiempos entre pruebas, en tanto variables de decisión, siguen una determinada función de distribución. Para describir la variabilidad de dichos variables de decisión se han empleado tres tipos de distribución diferentes: uniforme, normal y lognormal. También se ha empleado para todas las distribuciones, tres valores máximos de tolerancia admisible, correspondientes a unas tolerancias asumibles por la planta. Para resolver este problema de optimización multiobjetivo se ha empleado un algoritmo genético multiobjetivo que permite la consideración de las variables de decisión como variables aleatorias. Para administrar las incertidumbres se ha usado una aproximación basada en intervalos de tolerancia para asegurar un 
cierto nivel de tolerancia/confianza con un número mínimo de muestras.

Comparando los intervalos de tolerancia obtenidos para los criterios de indisponibilidad coste dependiendo del modelo de distribución asumido para los intervalos entre pruebas, se observa que, usando una distribución lognormal, los intervalos de tolerancia para indisponibilidad y costes son mayores que para el resto de distribuciones consideradas. También se observa que sólo si se permite una gran desviación del valor medio de la variable se aprecian intervalos de incertidumbre significativos para los criterios de indisponibilidad y coste.

Por otro lado, la comparación de las diferentes fronteras de Pareto alcanzadas en cada caso muestra que no hay diferencias significativas entre ellas. Es por ello que se puede concluir que usando la estrategia de optimización descrita, la frontera óptima de Pareto no tiene dependencia del modelo de distribución de las variables de decisión, ni del máximo valor del rango de tolerancias admitido. 


\subsection{Optimización considerando incertidumbre de parámetros.}

\subsubsection{Descripción del problema}

Dentro de las posibles fuentes de incertidumbre, se encuentran aquellas relativas los parámetros de los modelos utilizados en la cuantificación de los distintos criterios. El presente caso de aplicación considera esta fuente de incertidumbres dentro del problema de toma de decisiones multicriterio centrándose en los criterios de disponibilidad y coste, donde las variables de decisión son las pruebas y mantenimientos de los componentes del sistema de inyección a alta presión de una central nuclear (ver Figura 6-1). La estimación de la incertidumbre de los criterios de disponibilidad y coste en este caso participa en la definición tanto de los objetivos como de las restricciones del problema de optimización multiobjetivo.

Tal y como se formuló en el apartado 4.5.2 esta aproximación se encuadraría dentro del espectro de aproximaciones adversas al riesgo que mantiene bajo control el impacto de las incertidumbres en la optimización. Sin embargo, se impone el control del efecto de la incertidumbre en los criterios de fiabilidad y costes como restricciones fuertes dentro del conjunto de funciones restricción del POMO, aunque alternativamente se podrían imponer mediante restricciones débiles dentro de las funciones objetivo del POMO, tal y como se sugiere también en el apartado 4.5.2.

En este caso de aplicación no se intenta mantener la incertidumbre bajo un mínimo; el objetivo es mantener bajo control dicha incertidumbre ambos criterios, fiabilidad y coste, simultáneamente.

\subsubsection{Formulación del Problema}

El siguiente caso de aplicación se centra en el problema de la optimización de las actividades de pruebas y mantenimientos del sistema simplificado de inyección de alta presión de un reactor de agua a presión propuesto en la Figura 6-1 del capítulo 6. Este problema considera el efecto de la incertidumbre en la fiabilidad de los equipos en la optimización de las pruebas y mantenimiento, en particular, la incertidumbre epistémica en relación con los parámetros de fiabilidad que representan los fallos en espera y los fallos en demanda de los equipos. Para ello se ha usado un procedimiento de simulación por Monte Carlo y se ha utilizado las ventajas de los estadísticos de orden para proporcionar intervalos de tolerancia de distribución libre para la estimación de la indisponibilidad y costes basados en un número mínimo 
de ejecuciones necesarias $(N)$ para garantizar una probabilidad de contención o cobertura $(\gamma)$ con un nivel de confianza $(\beta)$. Para su resolución se ha utilizado los métodos planteados en el apartado 4.5.2.

Para este caso particular con modelos analíticos independientes del tiempo, se asume que el simulador es determinista, lo cual quiere decir que una vez fijados todos los parámetros de entrada al modelo se obtiene la misma salida, dentro de la exactitud computacional, para cada ejecución. Al mismo tiempo las entradas fluctúan de acuerdo a leyes de distribución reflejando la incertidumbre en las características de fiabilidad de los equipos. Consecuentemente, las variables de salida también fluctúan en ejecuciones repetidas.

La Tabla 6-1 y Tabla 6-2 (apartado 6.2) muestran valores típicos de los datos de fiabilidad, pruebas, mantenimientos y costes individuales para bombas y válvulas del HPIS, necesarios para cuantificar la indisponibilidad del sistema y el coste anual del mismo.

En este caso de aplicación, de forma similar a lo realizado en el capítulo 6, los componentes del sistema se han agrupado según tres grupos de intervalos entre pruebas $(T 1, T 2, T 3)$ viables de ser implementadas en planta y en dos grupos de mantenimiento preventivos diferentes, uno para bombas y otro para válvulas, que se realizan con una periodicidad M1 y M2 respectivamente. La estrategia T1 de pruebas se aplica a las válvulas que aportan agua desde el tanque de agua de almacenamiento de recarga a la línea de aspiración de las tres bombas, V1 y V2, las válvulas V3 y V5 emplazadas en la descarga de las bombas PA y PC y a las tres bombas PA, PB y PC. La estrategia T2 se aplica solo a la válvula de interconexión V4. Finalmente, se han incluido dentro de la tercera estrategia de pruebas T3 las válvulas de interconexión V6 y V7. La Tabla 7-4 resume los diferentes tipos de estrategia de pruebas y mantenimientos. 
Tabla 7-4: Grupos de estrategias de intervalos entre pruebas y mantenimientos preventivos para el sistema de inyección de agua a presión.

\begin{tabular}{lllllllllll}
\hline $\begin{array}{l}\text { Estrategias de } \\
\text { pruebas y } \\
\text { mantenimiento }\end{array}$ & PA & PB & PC & V1 & V2 & V3 & V4 & V5 & V6 & V7 \\
\hline T1 & $\mathrm{X}$ & $\mathrm{X}$ & $\mathrm{X}$ & $\mathrm{X}$ & $\mathrm{X}$ & $\mathrm{X}$ & & $\mathrm{X}$ & & \\
T2 & & & & & & & $\mathrm{X}$ & & & \\
T3 & $\mathrm{X}$ & $\mathrm{X}$ & $\mathrm{X}$ & & & & & & $\mathrm{X}$ & $\mathrm{X}$ \\
M1 & & & & $\mathrm{X}$ & $\mathrm{X}$ & $\mathrm{X}$ & $\mathrm{X}$ & $\mathrm{X}$ & $\mathrm{X}$ & $\mathrm{X}$ \\
M2 & & & & & & & & & & \\
\hline
\end{tabular}

Finalmente, las diferentes estrategias de pruebas deben satisfacer las siguientes relaciones, de acuerdo a la política actual de planificación de pruebas para un HPIS típico de cualquier central nuclear,

$\mathrm{T} 2=\mathrm{k} 2 \cdot \mathrm{T} 1$

$\mathrm{T} 3=\mathrm{k} 3 \cdot \mathrm{T} 1$

donde $k 2$ y k3, determinan la relación de proporcionalidad de los intervalos entre pruebas de los grupos 2 y 3 con el intervalo entre pruebas para el grupo 1.

Como esta aplicación se centra en la optimización de los intervalos entre pruebas y mantenimientos preventivos del HPIS simplificado y basados en criterios de indisponibilidad y coste, $\mathrm{U}+\mathrm{C}$, el vector de variables de decisión puede codificarse como:

$\mathrm{x}=\{\mathrm{T} 1, \mathrm{k} 2, \mathrm{k} 3, \mathrm{M} 1, \mathrm{M} 2\}$

La Tabla 7-5 presenta las variables de decisión, sus valores iniciales y el rango de variación permitido. También se adoptan como restricciones, $U_{L}=$ 5 th_pctil $\left(U\left(x_{i}\right)\right)$ y $C_{L}=5$ th_pctil $\left(C\left(x_{i}\right)\right)$. 
Tabla 7-5: Condiciones iniciales y rango de valores permitidos para las variables de decisión.

\begin{tabular}{|c|c|c|c|c|}
\hline \multirow{2}{*}{$\begin{array}{l}\text { Vector de } \\
\text { decisión }\end{array}$} & \multicolumn{3}{|c|}{ Rango } & \multirow{2}{*}{$\begin{array}{l}\text { Valor inicial } \\
x_{i}\end{array}$} \\
\hline & Min & Max & $\Delta$ & \\
\hline T1 (días) & 1 & 365 & 1 & 91 \\
\hline $\mathrm{k} 2=\mathrm{T} 2 / \mathrm{T} 1$ & 1 & 10 & 1 & 1 \\
\hline $\mathrm{k} 3=\mathrm{T} 3 / \mathrm{T} 1$ & 1 & 10 & 1 & 1 \\
\hline M1(días) & 1 & 3650 & 1 & 8 \\
\hline M2(días) & 1 & 3650 & 1 & 180 \\
\hline
\end{tabular}

En el capítulo 3 se proporciona una profunda formulación de los modelos necesarios y datos a ser evaluados en el criterio de decisión $A[U]+C$. Este ejemplo de aplicación adopta los modelos y datos de $U(x)$ y $C(x)$ que encontramos en dicha referencia y que son necesarios para cuantificar la función objetivo y de restricción. Para obtener la función de indisponibilidad del sistema, se desarrolla el árbol de fallos del sistema y se cuantifica usando la aproximación de conjuntos mínimos de corte (Minimal Cut Set, MCS) y las ecuaciones (3-12), (3-13), (3-14) y (3-28). En lo relacionado la función de costes, el modelo correspondiente ha obtenido añadiendo los costes asociados con pruebas, mantenimiento preventivo, reparaciones y reemplazamientos a partir de las ecuaciones (3-17) a (3-22). Los modelos dependen explícitamente de los parámetros que actuarán como variables de decisión en el vector de decisión x. Sin embargo, ambos modelos se han de adaptar aquí para incorporar el efecto de la incertidumbre como se ve a continuación.

Asimismo la consideración de la incertidumbre epistémica al separar la probabilidad de fallo del componente en su componente por demanda y la tasa de fallos se basa en la formulación planteada en el apartado 3.8, y concretamente mediante las expresiones para el fallo por demanda, $\rho$, dada por (3-32), y la tasa de fallo, $\lambda_{0}$, dada por (3-33).

De estas ecuaciones los valores de $\rho_{m}$ y $\lambda_{0 m}$ se pueden obtener como sigue. Primero, la no fiabilidad de las bombas y válvulas, $u_{r}\left(x_{i}\right)$, se obtiene usando las ecuaciones (3-28) a (3-30) para los valores originales tomados de la Tabla 6-1 (apartado 6.2) y los valores iniciales de $x_{i}$ (actuales) de la Tabla 7-5. $\rho_{m}$ 
se obtiene entonces usando las mismas ecuaciones y la $u_{r}\left(x_{i}\right)$ previamente obtenida cambiando los parámetros inciertos $\rho$ y $\lambda_{0}$ por las correspondientes expresiones (3-32) y (3-33) para el caso particular cuando $\theta=1$. Alternativamente, $\lambda_{o m}$ se obtiene usando las mismas ecuaciones y la $u_{r}\left(x_{i}\right)$ previamente calculada cambiando los parámetros inciertos $\rho$ y $\lambda_{0}$ por las correspondientes expresiones (3-32) y (3-33) para el caso particular $\theta=0$. La Tabla 7-6 resume los valores obtenidos para las válvulas y bombas del HPIS.

Tabla 7-6: Resultados para los valores máximos de $\rho_{m}$ y $\lambda_{0 m}$.

\begin{tabular}{llll}
\hline Equipos & $\mathrm{u}_{\mathrm{r}}\left(\mathrm{x}_{\mathrm{i}}\right)(-)$ & $\rho_{\mathrm{m}}(-)$ & $\lambda_{0 \mathrm{~m}}\left(\mathrm{~h}^{-1}\right)$ \\
\hline Válvulas & $1,92 \mathrm{E}-2$ & $8,1 \mathrm{E}-3$ & $7,47 \mathrm{E}-6$ \\
Bombas & $4,92 \mathrm{E}-3$ & $4,764 \mathrm{E}-3$ & $4,38 \mathrm{E}-6$ \\
\hline
\end{tabular}

\subsubsection{Método de resolución y resultados}

Se van a usar las aproximaciones AGSO y AGMO descritas en el apartado 4.5.2 como técnicas de optimización. La Tabla 7-7 muestra los valores adoptados para los parámetros relevantes de ambas aproximaciones.

En concordancia con la Tabla 7-7 la enumeración de todo el espacio de decisión requeriría la ejecución del siguiente número máximo de combinaciones/evaluaciones de las funciones objetivo cada vez,

$\mathrm{E} \leq \mathrm{pS}(1+\mathrm{G} \cdot \mathrm{pR})=100(1+50 \cdot 0,95)=4850$

siendo $p S$ el tamaño de la población, $p R$ la probabilidad de reemplazamiento en cada generación y tomando el número de generaciones estudiadas, $G$, como 50. Este número máximo de combinaciones/evaluaciones resulta ser una pequeña fracción del máximo número de evaluaciones por enumeración dado por el número total de combinaciones para cada posible valor del vector de decisión $\mathrm{x}$.

$\mathrm{E}_{\max }=365 \mathrm{EEx} 3650 \times 3650 \approx 4,86 \mathrm{E} 11$ 
Además, el número correspondiente de ejecuciones (historias) del modelo de simulación $\mathrm{A}[\mathrm{U}]+\mathrm{C}$, necesarios cuando el número de pruebas por evaluación es de, por ejemplo, $N=459$ (apartado 4.5.2) es aproximadamente:

$\mathrm{R}=\mathrm{N} \cdot \mathrm{E}=459 \cdot 4850=2226150$

Tabla 7-7: Parámetros adoptados para los AGMO y SOGA particulares

\begin{tabular}{lll}
\hline Parámetro & AGSO & AGMO \\
\hline Algoritmo Genético & AGSO-U y AGSO-C & AGMO-H y AGMO-E \\
Generaciones (G) & Variable $\{5,10,30,50,100\}$ \\
Número de pruebas (N) & Variable $\{64,130,458,662,10000\}$ \\
Tamaño Población (pS) & 100 & \\
Inicialización & Aleatoria con valores iniciales \\
Mating selection & Selector de torneo & \\
Operador de cruces & Un punto de cruce & \\
Tasa de cruces & 0,9 & \\
Operador de Mutación & Mutación flip & \\
Tasa de Mutación & 0,2 & Mutación Gausiana Real \\
Probabilidad de & 0,95 & Dominancia de Pareto \\
Reemplazamiento )pR) & \multicolumn{1}{l}{ basado en SPEA2 } \\
Evaluación & Ajuste & No escalado \\
Manejo de Restricciones & Penalización y rechazo dinámica \\
Escala de ajuste & Escalado lineal & \\
Terminación & Generaciones & \\
Tamaño Externo & 100 &
\end{tabular}




\subsubsection{Resolución basada en AGMO-H}

La Figura 7-6 representa el gráfico de resultados U-C encontrados para el AGMO-H introducido en la sección 4.5.2.2.1 y que hace referencia al AGMO utilizando los límites más altos de tolerancia. Muestra el valor medio y los intervalos de confianza correspondientes a los percentiles $5 \%$ y $95 \%$ obtenidos después de ejecutar una simulación de Montecarlo para una gran cantidad de pruebas para cada solución propuesta por el AGMO-H. Los resultados mostrados han sido calculado usando un AGMO-H con una población de tamaño $p S=100$ individuos para $G=50$ generaciones con probabilidad de reemplazo de $p R=0,95$. Para obtener el intervalo de tolerancia para cada individuo en el caso base, el individuo se ha ejecutado para $N=662$ pruebas, correspondiente a la pareja de confianza/tolerancia del dominio de espacio de búsqueda de 0,99/0,99 para el caso de considerar la indisponibilidad del sistema y coste como salidas dependientes. En la Figura 7-6, se ha obtenido una frontera de Pareto para un caso determinista sin incertidumbre en los parámetros $(\theta=0,5$ constante). Se puede observar que hay un vacío de soluciones en la región con alta incertidumbre (esto es, baja indisponibilidad y alto coste). Esta característica es independiente del número de ejecuciones ya que se ha comprobado que presenta similares comportamientos con diferente número de las mismas.

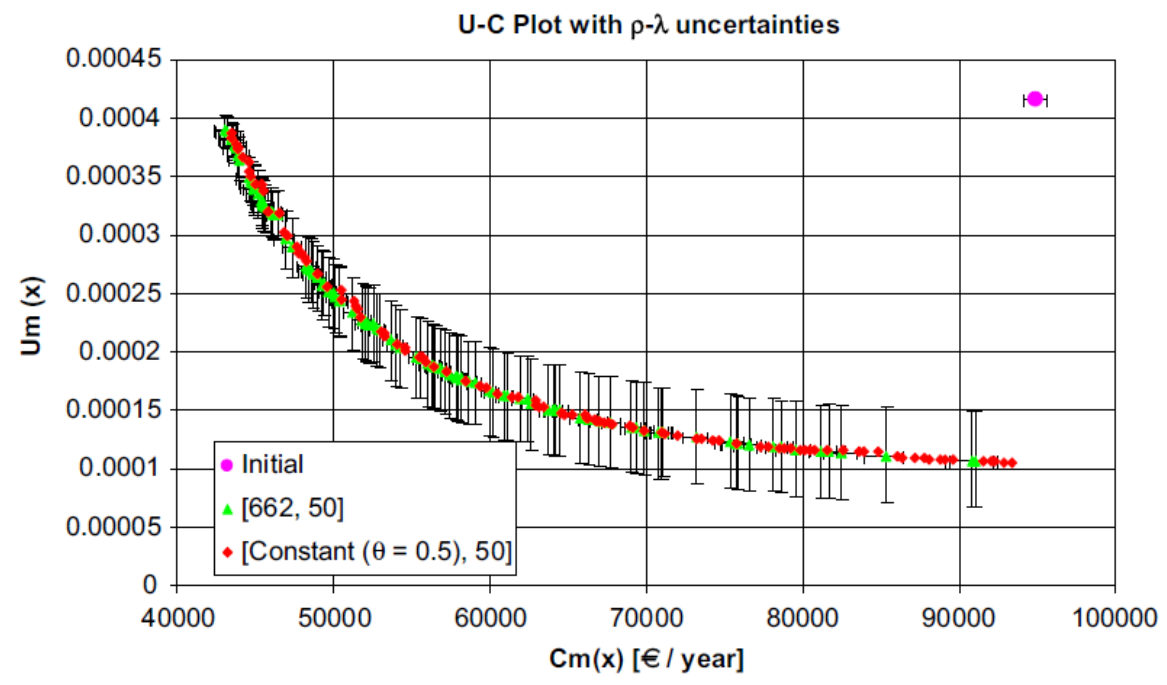

Figura 7-6: Gráfica U-C de la incertidumbre de los resultados considerando dependencia entre $\mathrm{U}$ y $\mathrm{C}$ 
A continuación se presentan los resultados del análisis de sensibilidad ejecutados según el número de pruebas $N$ por evaluación y el número de generaciones $G$ del AGMO-H para la salidas dependientes $U$ y $C$. La Figura 7-7, Figura 7-8 y Figura 7-9 muestran los gráficos U-C de los valores medios correspondientes a las soluciones encontradas después del análisis de sensibilidad en el número de pruebas $N\{64,130,662,10000\}$ para tres conjuntos de generaciones $G\{10,50,100\}$, representado una evolución corta, mediana y larga respectivamente.

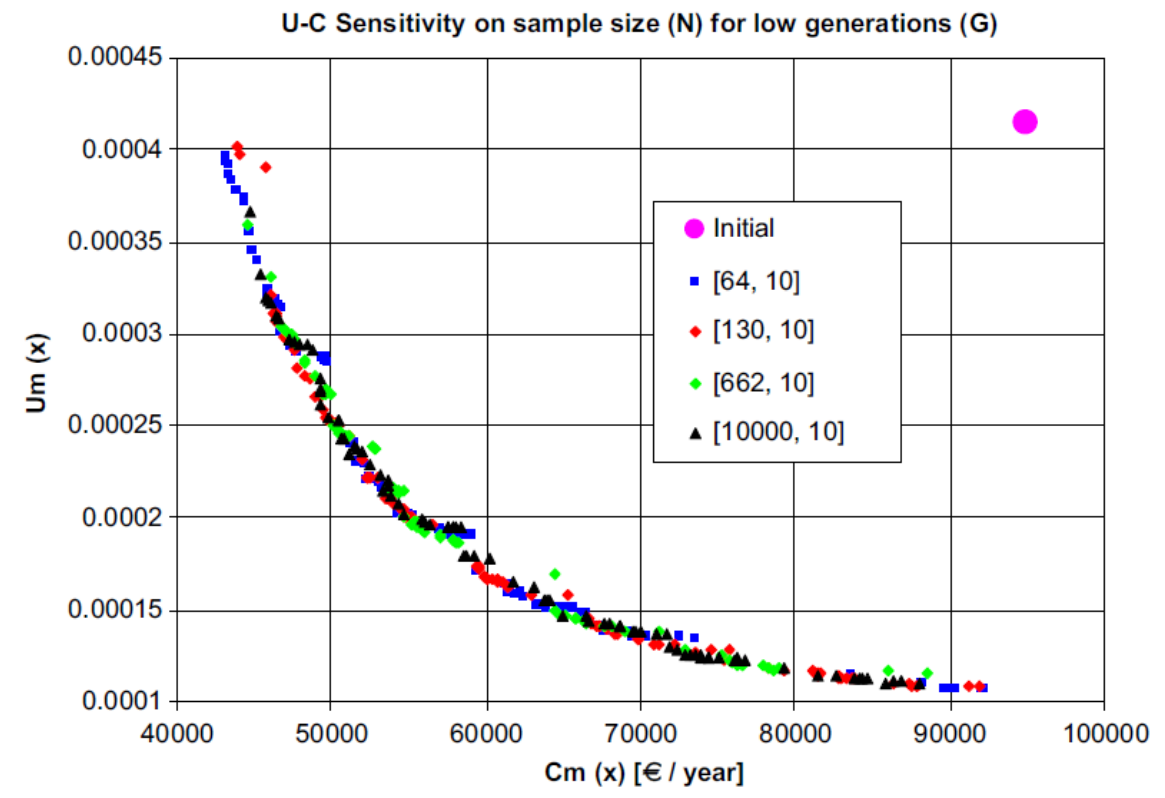

Figura 7-7: Evolución Corta ( $\mathrm{G}=10)$ 
Optimización multiobjetivo de la vigilancia y mantenimiento a nivel de sistema considerando el efecto de las incertidumbres

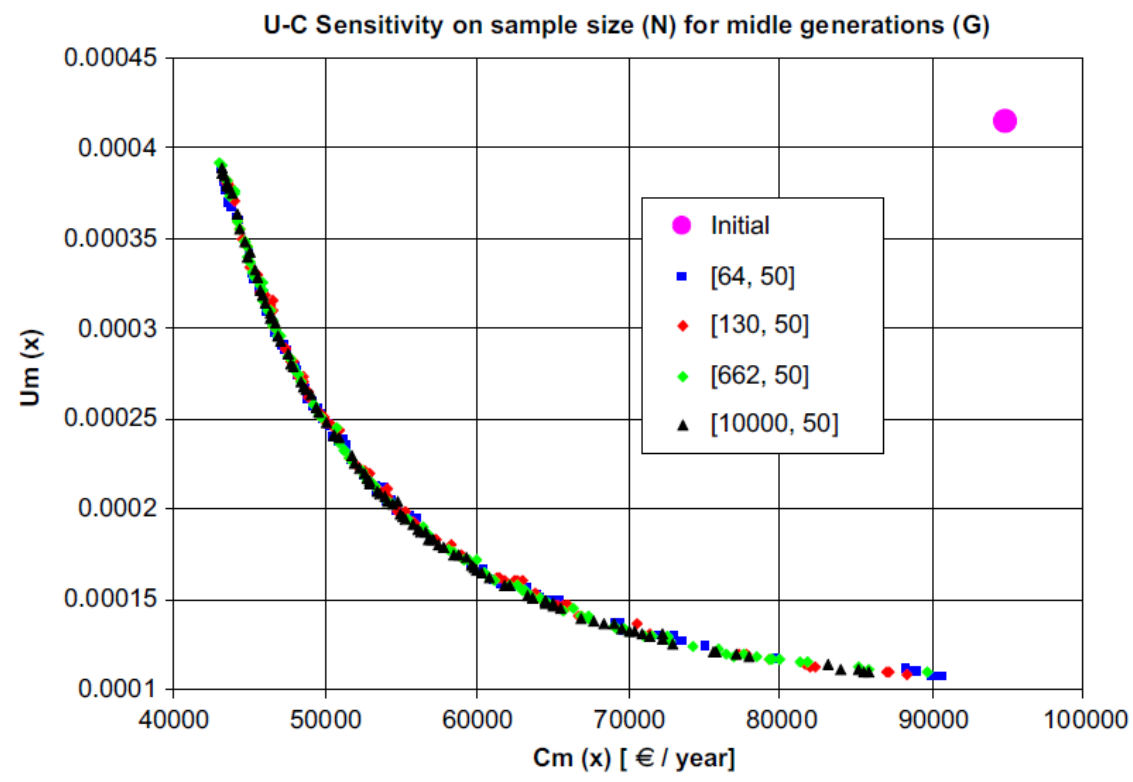

Figura 7-8: Evolución Mediana $(G=50)$

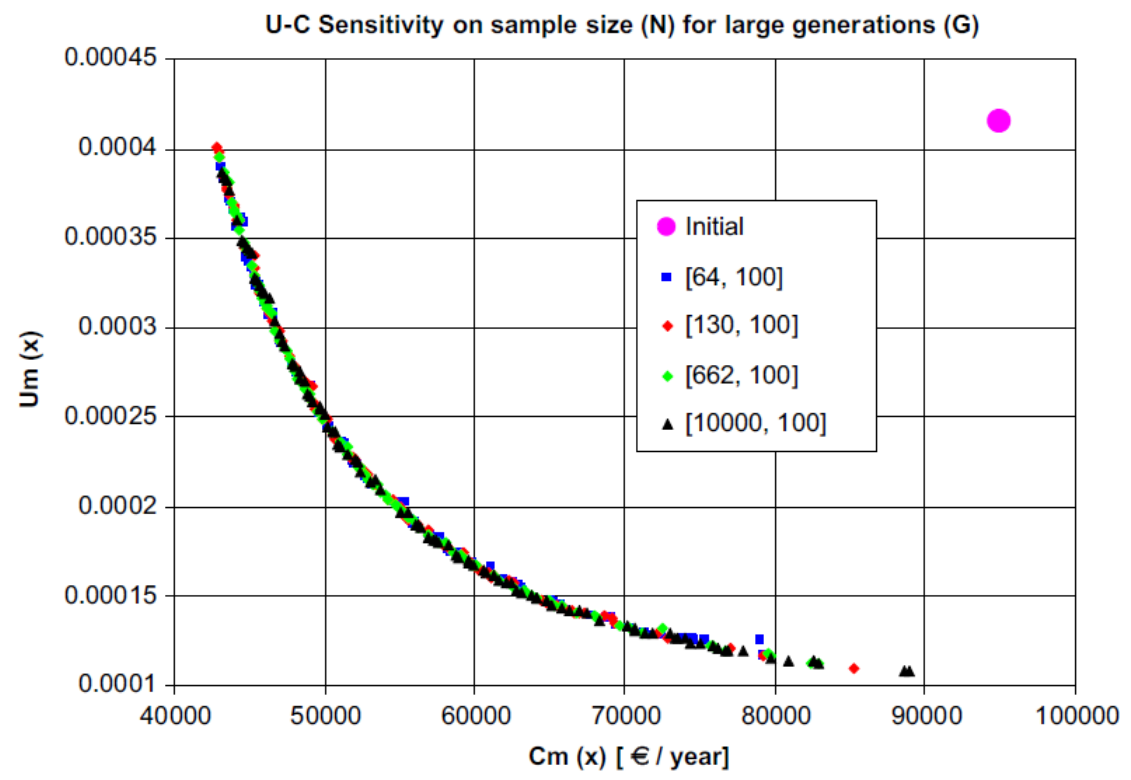

Figura 7-9: Evolución Larga (G=100) 
La Figura 7-7 muestra que los resultados conseguidos después de una evolución corta proporcionan una frontera de Pareto completamente definida aunque aumente el número de pruebas. Sin embargo de la Figura 7-8 y Figura 7-9 se puede observar que a medida que el número de pruebas $N$ aumenta, mejora la definición de la frontera de Pareto obtenida para una evolución razonable, media o larga. La selección del número máximo de pruebas es muy importante ya que parece que un mayor número de pruebas proporciona mejores resultados pero exige un importante coste computacional.

A partir de estos estudios de sensibilidad surge la cuestión del efecto que el aumento del número de generaciones tiene con un aumento similar en el número de pruebas.

La Figura 7-10 trata de resumir tal comparación basada en los valores medios de los resultados encontrados. Así, ambas parejas $[N, G]$ dadas por $[50,662]$ y $[10000,30]$ proporcionan resultados similares comparando las respectivas fronteras de Pareto. Sin embargo se puede apreciar que la primera pareja implica $R=3,21 \cdot 10^{6}$ historias (ejecuciones de los modelos de simulación de dependencia y coste) frente al valor de $R=2,95 \cdot 10^{7}$ para la última pareja. Globalmente parece una mejor inversión en términos de coste computacional, implicar un mayor número de generaciones en lugar de una mayor precisión en la cuantificación del criterio de decisión para la solución candidata, esto es un número mayor de pruebas. 


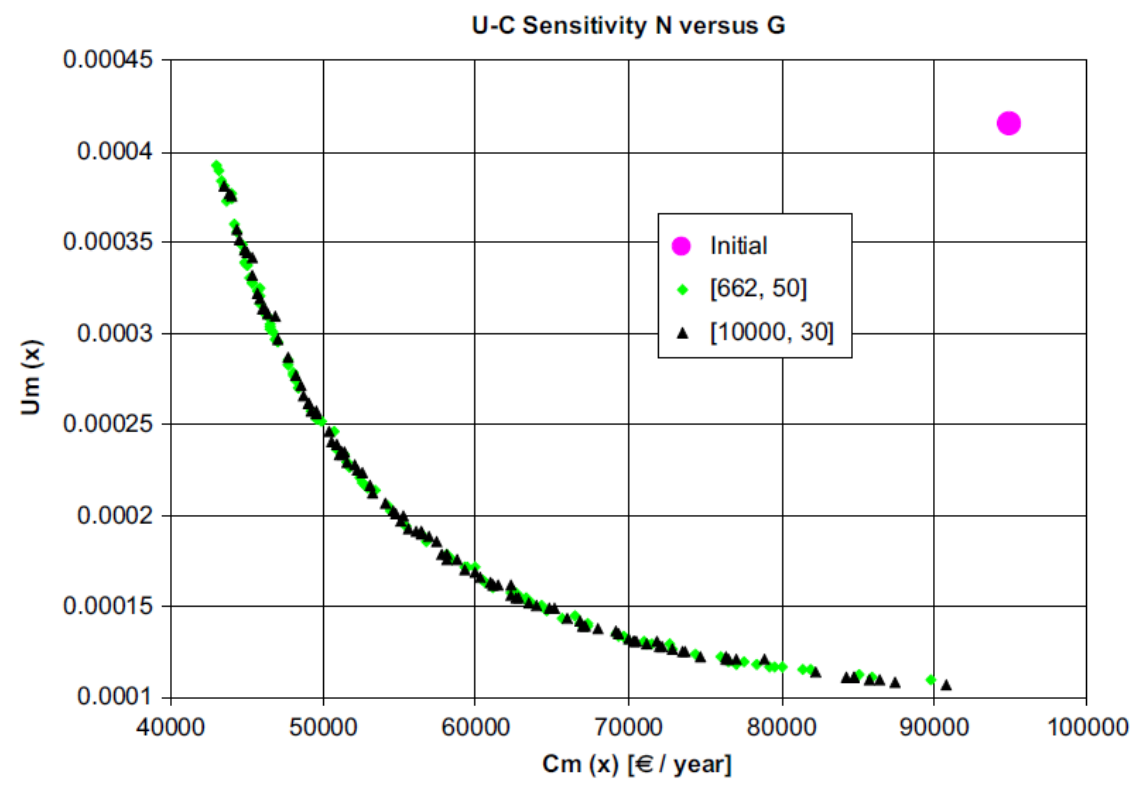

Figura 7-10: Gráfica U-C de resultados medios del análisis de sensibilidad de G frente a $\mathrm{N}$ para dos parejas comparables.

De todo esto se puede concluir que la evolución es preferible en comparación a la precisión. Esta conclusión va en la misma dirección que la previa considerando independencia en los criterios de indisponibilidad y coste. Sin embargo aparecen efectos secundarios. Observando la Figura 7-8 y Figura 7-9 se aprecia que a medida que el número de generaciones implicadas, $G$, aumenta, la frontera de Pareto se mueve hacia la solución con menos incertidumbre, esto es, hacia valores de coste menores, y este efecto es más evidente cuando el número de pruebas $\mathrm{N}$ es bajo, esto es, obteniendo estimaciones más rudas en el criterio de decisión, mientras no se observa cuando $N$ es alto. Es por ello que hay una necesidad de buscar soluciones para resolver este problema como a continuación se presenta.

\subsubsection{Resolución basada en AGMO-E}

La Figura 7-11 representa un gráfico U-C con los valores medios encontrados con el AGMO-E introducido en el apartado 4.5.2.2.1 y que hace referencia al AGMO utilizando valores medios con límites de tolerancia superiores para los criterios de decisión en comparación con los resultados obtenidos usando la 
aproximación AGMO-H del apartado anterior. La Figura 7-11 muestra que los nuevos resultados son similares a los presentados en la Figura 7-6 excepto que la frontera de Pareto se distribuye mejor ahora, lo que sugiere que la formulación de las funciones multiobjetivo basadas en valores medios y manteniendo la formulación de las restricciones basadas en intervalos de tolerancias soluciona el problema planteado anteriormente. Esto se confirma con la Figura 7-12 que representa un gráfico U-C con los resultados del análisis de sensibilidad ejecutados para diferentes números de generaciones, $G$, del AGMO para salidas dependientes $U$ y $C$, y manteniendo constante el número de pruebas $\mathrm{N}=662$,

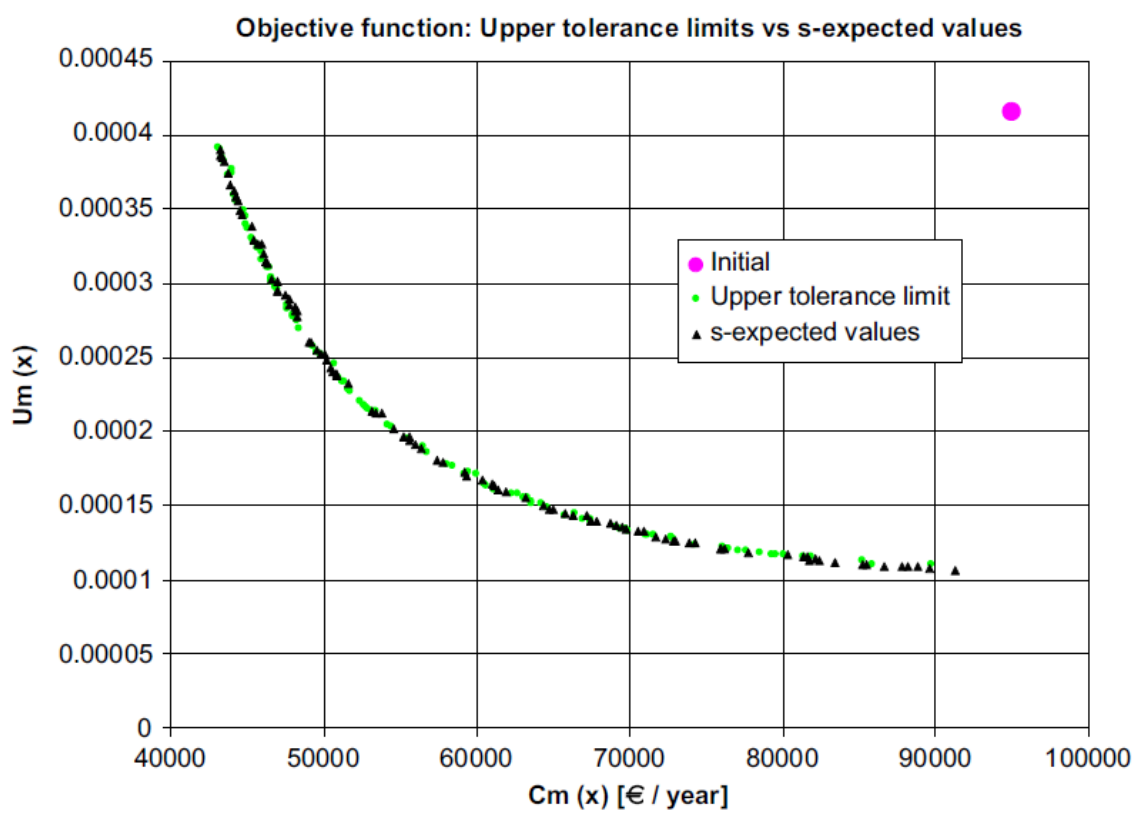

Figura 7-11: Gráfico U-C de resultados con incertidumbre considerando dependencia entre $\mathrm{U}$ y $\mathrm{C}$ y funciones objetivos basadas en valores medios. 


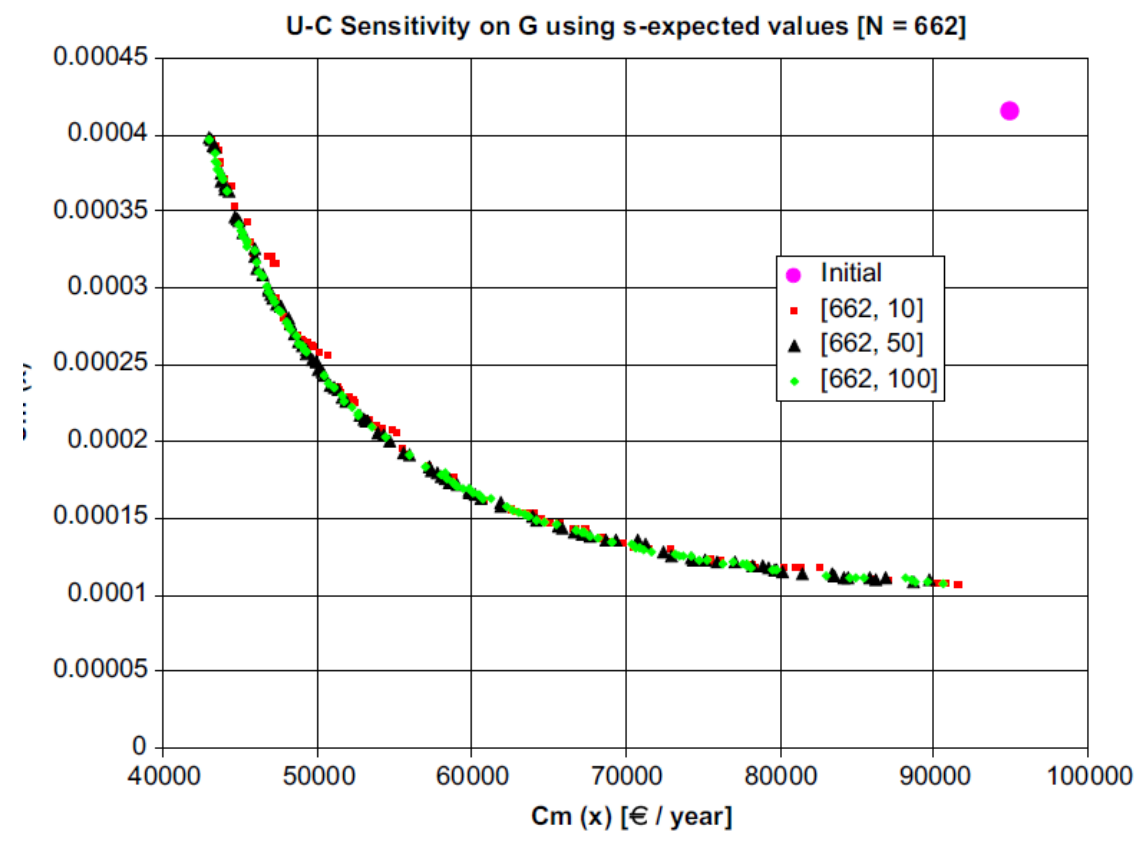

Figura 7-12: Gráfico U-C de resultados con incertidumbre del análisis de sensibilidad para $\mathbf{G}$ con $\mathbf{N}$ constante y considerando dependencia entre $\mathrm{U}$ y $\mathrm{C}$ y funciones objetivo basados en valores medios

\subsubsection{Resolución basada en AGSO}

La Figura 7-13 representa un gráfico U-C de resultados medios encontrado para el AGMO-H en comparación con los resultados obtenidos por transformación del POMO original en dos POSO extremos. Se ha usado la aproximación AGSO descrita anteriormente (apartado 4.5.2.2.2) en ambos casos, esto es AGSO-U y AGSO-C. Dicha aproximación se basa en el WEBA que permite definir la función objetivo mediante la expresión (4-32).

La Figura 7-13 muestra que el objetivo que se busca con el método AGSO se consigue ya que el AGSO se centra en una región particular, AGSO-U y AGSO-C en este caso, y entonces la búsqueda está mejor guiada y ejecuta más rápido hacia esta región. Esta aproximación, proporciona una parte de la frontera de Pareto en una cierta región, incluso si esta región incorpora una gran incertidumbre en los criterios de decisión. La Figura 7-14 y Figura 7-15 representan el gráfico U-C con los resultados del análisis de sensibilidad para un numero de generaciones $G$ para ambos casos de AGSO-U y AGSO-C 
para el mismo número de pruebas $N=662$.

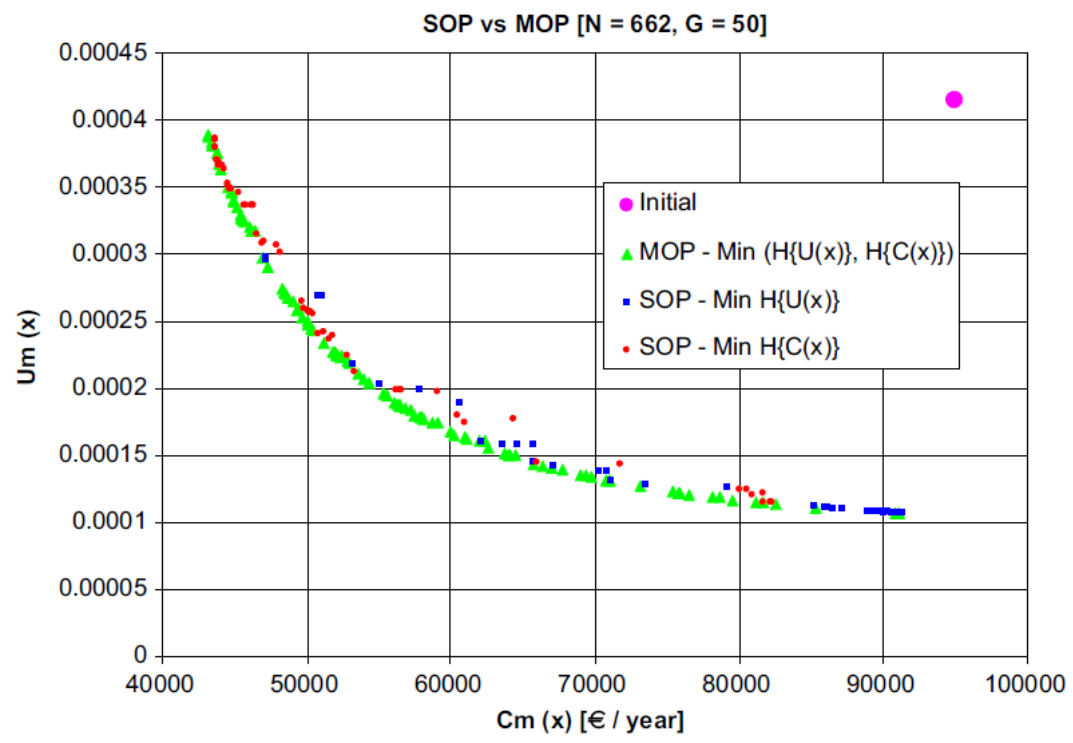

Figura 7-13: Gráfico U-C de resultados medios para los problemas de optimización simple y múltiple

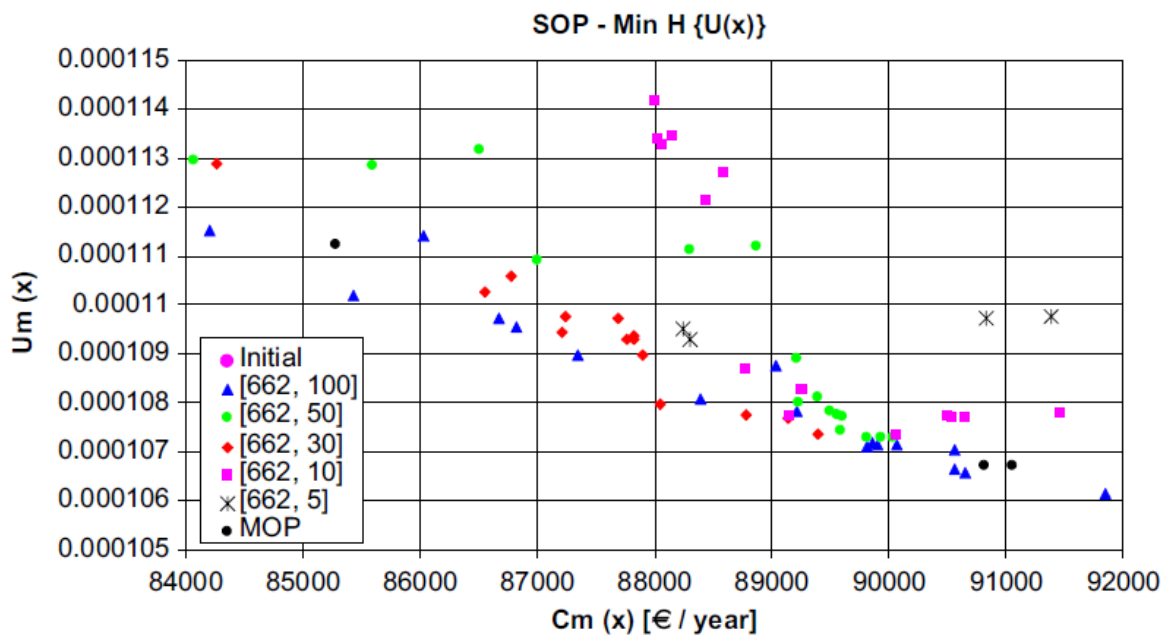

Figura 7-14: Análisis de sensibilidad de los valores medios en N para POS de minimización del límite superior de tolerancia para indisponibilidad de sistemas, esto es AGSO-U (solo se muestra la zona de interés) 
La Figura 7-13, Figura 7-14 y Figura 7-15 muestran que el AGSO llega al mayor número de buenas soluciones en la región de interés, ya sea mínima indisponibilidad o mínimo coste, en comparación con el AGMO-H que resuelve el POMO directamente. Además, el AGSO requiere menos evoluciones, $G=30$ o menos, que el AGMO-H original, que implicaba $G=50$ para llegar a similar número de soluciones buenas en la región de interés. Además el procedimiento de resolución es más robusto ya que exhibe una ejecución que es coherente con el comportamiento esperado ya que largas evoluciones conducen a mejores soluciones en la región de interés no importa como de inciertas son las soluciones en dicha región. Es por todo ello que esta alternativa resuelve el problema planteado con anterioridad.

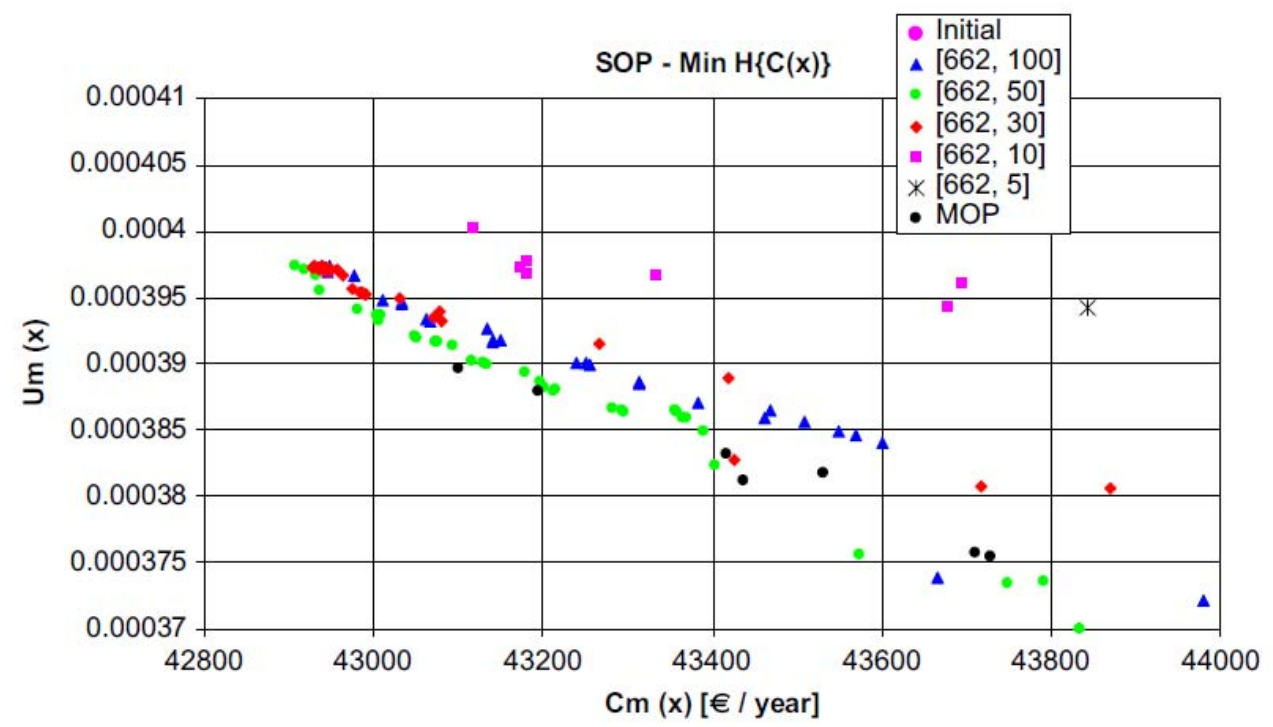

Figura 7-15: Análisis de sensibilidad de los valores medios en $\mathrm{N}$ para POS de minimización del límite superior de tolerancia para el coste del sistema, esto es AGSO-C (solo se muestra la zona de interés)

\subsubsection{Discusión}

En este caso de aplicación se han propuesto varias aproximaciones para tratar las incertidumbres dentro de la toma de decisiones multicriterio basadas en la indisponibilidad y coste. El problema se ha formulado como un problema de optimización multiobjetivo, donde han actuado como variables de decisión las pruebas y mantenimiento, mientras que la estimación de 
indisponibilidad y coste considerando incertidumbres, han participado en la formulación de las funciones objetivo y restricciones. Esta aproximación entra dentro el campo de las aproximaciones adversas al riesgo, que mantiene bajo control el impacto de las incertidumbres. El control del efecto de la incertidumbre dentro de los criterios de fiabilidad y coste se impone como una restricción fuerte dentro del POMO.

Esta aproximación puede proveer de una herramienta rápida, robusta y poderosa para la optimización de la fiabilidad y costes de las pruebas y mantenimientos bajo incertidumbres por una combinación efectiva entre las simulaciones de Montecarlo y los AA.GG. basados en intervalos de tolerancia de distribución libre.

La comparación dentro del estudio de sensibilidad del número de generaciones frente al número de ejecuciones muestra que parece mejor una inversión, en términos de esfuerzo computacional, realizar un mayor número de generaciones $G$, en vez de exigir mayor precisión en la cuantificación de los criterios de fiabilidad y costes para una solución candidata, que requieren un mayor número de ejecuciones $N$. Además, como efecto colateral se observa, que la frontera de Pareto se desplazaba hacia soluciones con menos incertidumbre cuando $N$ y $G$ no se encuentran en equilibrio, se ha podido evitar con la adopción de las nuevas alternativas introducidas en la aplicación.

Se ha mostrado que el uso de un AGSO o el uso de un AGMO con tratamiento estadístico permite mejorar anteriores aproximaciones y al mismo tiempo minimizar el efecto negativo de no usar un par evolución-precisión bien ajustado.

En particular la aproximación AGSO se centra una región particular del espacio de búsqueda, por lo que la búsqueda está guiada y se evoluciona más rápido hacia la región de interés proporcionando una parte de la frontera de Pareto. Esto ocurre incluso para el caso cuando esta región incorpora una gran incertidumbre en el criterio de decisión. Además, la resolución se hace más robusta ya que la ejecución es más coherente con el comportamiento esperado, es decir una evolución más larga conduce a una solución mejor en la región de interés, sin importar cómo son de inciertas las soluciones en esa región.

Por tanto, la ventaja de la aproximación AGSO se hace evidente. Sin embargo, la aproximación AGMO-E proporciona una mejor distribución del conjunto de soluciones. Las soluciones encontradas con el AGMO-E muestran que los nuevos resultados son similares a los previos obtenidos por 
un AGMO-H excepto que la frontera de Pareto ahora se encuentra mejor distribuida, lo que demuestra que la formulación de las funciones multiobjetivos basadas en valores medios y manteniendo la formulación de las restricciones basadas en los intervalos de tolerancia también resuelve los efectos negativos que se habían presentado inicialmente. 


\subsection{Optimización considerando incertidumbres de modelo de mantenimiento imperfecto.}

\subsubsection{Descripción del problema}

Uno de las incertidumbres más importantes inherentes a los modelos usados en los problemas de optimización se refiere al efecto que las actividades de mantenimiento tienen sobre el estado de un componente. En general, el efecto del mantenimiento que se lleva a cabo sobre un componente se puede simular mediante un modelo de mantenimiento imperfecto. La selección de un modelo adecuado para simular el efecto de un mantenimiento imperfecto se basa normalmente en un juicio de expertos. Además, el modelo de mantenimiento imperfecto suele asumir que cada actividad mejora el estado del componente en algún grado, dependiendo del parámetro de efectividad. Comúnmente, en la literatura, la efectividad del mantenimiento se estima en base a un conjunto de sugerencias de expertos y/o por estimación en base a datos reales, y se considera constante en base al comportamiento esperado. Esto puede no ser realista en muchas situaciones en las que la efectividad del mantenimiento no se conoce, especialmente en situaciones donde los datos son limitados y la incertidumbre es bastante grande, y puede tener una influencia decisiva en los resultados del proceso de toma de decisiones (Bunea 2002) y (Wu 2005).

La presente aplicación trata el problema de optimización de pruebas y mantenimientos basado en criterios de indisponibilidad y coste con incertidumbre epistémica asociada al modelo de mantenimiento imperfecto y al parámetro de efectividad del mantenimiento, usando las aproximaciones expuestas en el apartado 3.8.

\subsubsection{Formulación del problema}

El siguiente caso de aplicación se centra en el problema de la optimización de las actividades de pruebas y mantenimiento del sistema simplificado de inyección de alta presión de un reactor de agua de agua a presión propuesto en el capítulo 6 (ver Figura 6-1. Este problema considera el efecto de la incertidumbre tanto en el modelo de mantenimiento imperfecto como en el parámetro, $\varepsilon$, de efectividad del mismo, bajo criterios de coste e indisponibilidad de sistemas.

La Tabla 6-1 y Tabla 6-2 (apartado 6.2) muestran valores típicos de los datos de fiabilidad, pruebas, mantenimientos y costes individuales para bombas y 
válvulas del HPIS, necesarios para cuantificar la indisponibilidad del sistema y el coste anual del mismo.

La Tabla 7-4 muestra una posible estrategia de intervalos de pruebas (TI) para válvulas y bombas agrupadas en tres intervalos entre pruebas diferentes $\mathrm{T}\{\mathrm{T} 1, \mathrm{~T} 2, \mathrm{~T} 3\}$, viables de ser implementadas en planta. La misma tabla muestra que se han considerado dos grupos de mantenimientos preventivos, uno para bombas y otro para válvulas, las cuales se ejecutan con periodicidad M1 y M2 respectivamente.

Como esta aplicación se centra en la optimización de los intervalos entre pruebas y mantenimientos preventivos del HPIS simplificado y basados en criterios de indisponibilidad y coste, $\mathrm{U}+\mathrm{C}$, el vector de variables de decisión puede codificarse como:

$\mathrm{x}=\{\mathrm{T} 1, \mathrm{k} 2, \mathrm{k} 3, \mathrm{M} 1, \mathrm{M} 2\}$

donde, de acuerdo con la actual política de mantenimiento para un HPIS típico de una central nuclear, se aplica la siguiente relación $T 2=k 2^{\star} T 1$ y $T 3=k 3^{*} T 1$, donde $k 2$ y $k 3$, determinan la relación de proporcionalidad de los intervalos entre pruebas de los grupos 2 y 3 con el intervalo entre pruebas para el grupo 1.

La Tabla 7-5 presentan los cinco parámetros considerados como variables de decisión en este estudio, sus valores iniciales y el rango de variación permitido.

En el capítulo 3 se proporciona una profunda formulación de los modelos necesarios y datos a ser evaluados en el criterio de decisión $A[U]+C$. Este ejemplo de aplicación adopta los modelos y datos de $U(x)$ y $C(x)$ que encontramos en dicha referencia y que son necesarios para cuantificar la función objetivo y de restricción. Para obtener la función de indisponibilidad del sistema, se desarrolla el árbol de fallos del sistema y se cuantifica usando la aproximación de conjuntos mínimos de corte (Minimal Cut Set, MCS) y las ecuaciones (3-12), (3-13), (3-14) y (3-28). En lo relacionado la función de costes, el modelo correspondiente ha obtenido añadiendo los costes asociados con pruebas, mantenimiento preventivo, reparaciones y reemplazamientos a partir de las ecuaciones (3-17) a (3-22).

Para este caso concreto se considera asimismo la efectividad del mantenimiento $(\varepsilon)$ y la incertidumbre en el modelo de mantenimiento imperfecto (PAS o PAR), lo que significa que la cuantificación de la 
indisponibilidad y el coste puede fluctuar.

En (Martorell 2004) la efectividad del mantenimiento se consideraba un valor constante. Como las actividades planificadas de mantenimiento de bombas y válvulas son diferentes, la efectividad del mantenimiento puede ser, en principio, diferente para cada equipo. Por eso se han considerado dos variables distintas de efectividad de los mantenimientos, $\varepsilon_{v}$ y $\varepsilon_{p}$, uno para válvulas y otro para las bombas, representadas por sendas variables aleatorias. Como se ve en (Helton 2004) al desconocer la función de distribución del parámetro, se usa una distribución uniforme para caracterizar la incertidumbre en ausencia de información para diferenciar la credibilidad de diferentes alternativas, y también es apropiado si se cree que cada uno de los valores del intervalo es igualmente probable. Así en este caso, se asume que la efectividad del mantenimiento para válvulas y bombas, $\varepsilon_{v}$ y $\varepsilon_{p}$, son variables aleatorias con una distribución uniforme en los siguientes rangos:

$$
\begin{aligned}
& \varepsilon_{\mathrm{p}}=\text { Uniform }[0.5,0.9] \\
& \varepsilon_{\mathrm{v}}=\text { Uniform }[0.5,0.8]
\end{aligned}
$$

Como $\varepsilon_{p}$ y $\varepsilon_{v}$ participan en la formulación de la indisponibilidad del sistema y coste, los cuales sucesivamente actúan como funciones objetivo y restricciones, conducen a la formulación del problema multiobjetivo enfocado a la optimización del intervalo entre pruebas, $T$, y mantenimiento, $M$, bajo objetivos y restricciones inciertas dados por las ecuaciones (4-29) y (4-30).

Se ha usado un AGMO adaptado para implementar la aproximación basada en el método de intervalos de tolerancia para manejar las incertidumbres en la optimización informada en $U+C$ de pruebas y mantenimientos (apartado 4.5.2). La Tabla 7-8 muestra los valores adoptados para los parámetros más relevantes del AGMO. En este caso de aplicación $U(x)$ y $C(x)$ se asumen como independientes para seleccionar el número de pruebas en el muestreo de Montecarlo que definen el intervalo de tolerancia/confianza requerido. 
Tabla 7-8: Parámetros adoptados para el AGMO ajustado al método de intervalos de tolerancia.

\begin{tabular}{ll}
\hline Parámetro & Valor \\
\hline Generaciones (G) & 150 \\
Número de pruebas (N) & 459 (para $/ / \beta=0,99 / 0,99)$ \\
Tamaño Población (pS) & 100 \\
Inicialización & Aleatoria con valores iniciales \\
Mating selection & Selector de torneo \\
Operador de cruces & Un punto de cruce \\
Tasa de cruces & 0,9 \\
Operador de Mutación & Mutación Gausiana Real \\
Tasa de Mutación & 0,2 \\
Probabilidad de Reemp (pR) & 0,95 \\
Evaluación & Dominancia de Pareto basado en SPEA2 \\
Manejo de Restricciones & Penalización y rechazo dinámica \\
Escala de ajuste & Reparto \\
Terminación & Generaciones \\
Tamaño Externo & 100 \\
\hline
\end{tabular}

El tiempo de ejecución depende del correspondiente número de ejecuciones (R) de la simulación, el cual se puede evaluar aproximadamente como:

$\mathrm{R}=\mathrm{N} \cdot \mathrm{E}$

donde $N$ es el número de pruebas por evaluación, en este caso $N=459$, y $E$ el número total de evaluaciones de las soluciones candidatas llevadas a cabo por el AGMO. De acuerdo a los valores adoptados en la Tabla 7-8, el número de evaluaciones es aproximadamente:

$\mathrm{E} \leq \mathrm{pS} \cdot(1+\mathrm{G} \cdot \mathrm{pR})=100 \cdot(1+150 \times 0,95)=14350$ 
donde $p S$ es el tamaño de la población, $G$, es el número de generaciones desarrolladas por el $A G M O$ y $p R$ es la probabilidad de reemplazamiento de las poblaciones después de cada generación. Sustituyendo $N=459$ y $E=14350$ en (7-8) se obtiene:

$\mathrm{R} \leq 459 \cdot 100 \cdot(1+150 \times 0,95)=6586650$

\subsubsection{Método de resolución y resultados}

\subsubsection{Resultados de la optimización bajo incertidumbre en el parámetro $\varepsilon$.}

En primer lugar, se ha analizado la influencia de la incertidumbre en la efectividad del mantenimiento, parámetro $\varepsilon$, en el proceso de optimización, manteniendo sin cambios los modelos de mantenimiento imperfecto para bombas y válvulas, que siguen una aproximación PAS y PAR respectivamente.

La Figura 7-16 muestra los resultados obtenidos en el tratamiento de la incertidumbre en $\varepsilon_{p}$ y $\varepsilon_{v}$ comparado con los resultados obtenidos cuando la efectividad se mantiene constante. Se han considerado los dos casos extremos con la efectividad constante: (1) mínima efectividad, lo cual representa el peor mantenimiento posible, esto es $\varepsilon_{p}=\varepsilon_{v}=0,5$, y (2) máxima efectividad, lo cual representa el mejor mantenimiento posible cuando $\varepsilon_{p}=$ 0,9 y $\varepsilon_{v}=0,8$. Además, el modelo de mantenimiento imperfecto adoptado para todas la válvulas del sistema es la aproximación PAR, mientras que para las bombas se ha adoptado la aproximación PAS, ver ecuaciones (3-29) y (3-30) en el apartado 3.8.1.

Como muestra la Figura 7-16, cuando se considera la incertidumbre en la efectividad del mantenimiento $\varepsilon$, los valores óptimos para $U$ y $C$ se mantienen dentro de las curvas obtenidas para la dos situaciones extremas. También, en la misma figura se observa que el rango de incertidumbre para la indisponibilidad es mayor para aquellos puntos cercanos a la máxima indisponibilidad, mientras que la incertidumbre para el rango de coste presenta ligeras variaciones significativas. 


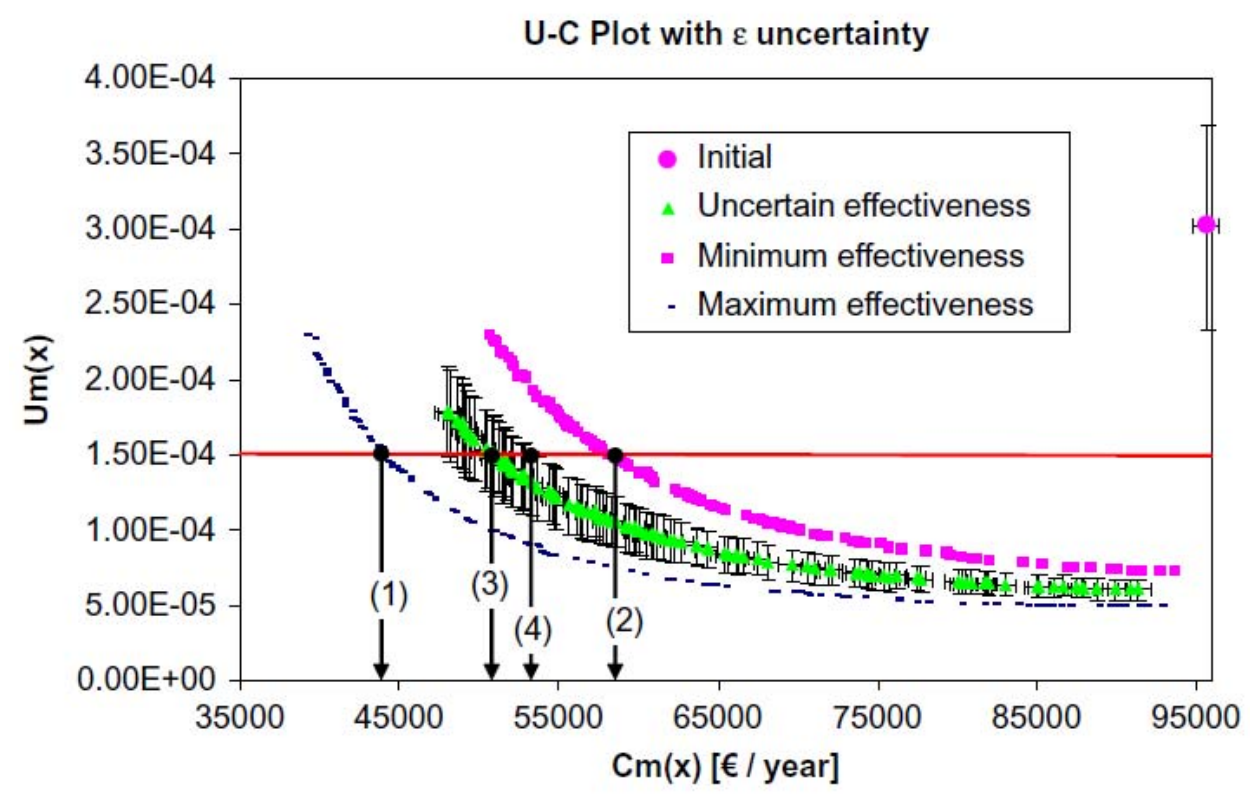

Figura 7-16: Resultados de la optimización considerando incertidumbre en el parámetro $\varepsilon$.

Los resultados mostrados en la Figura 7-16 sugieren diferentes posibilidades para tomar la decisión final. Así, suponiendo un nivel máximo en la indisponibilidad del sistema que puede ser asumible, por ejemplo $1,5 \cdot 10^{-4}$; la mejor opción desde el punto de vista del coste, mínimo coste, se encuentra considerando la solución obtenida con la máxima efectividad, punto (1) en Figura 7-16. Esta selección es una hipótesis poco conservadora, ya que estamos considerando un valor de efectividad máximo. En el otro extremo, si se considera el peor valor para la efectividad del mantenimiento, para la misma indisponibilidad, el coste mínimo aceptable es considerablemente mayor, punto (2) en la Figura 7-16.

Se obtiene una situación más realista cuando se considera la incertidumbre en la efectividad del mantenimiento en el proceso de optimización. La Figura 7-16 también muestra los resultados obtenidos en este caso. La frontera de Pareto con incertidumbres se localiza en la zona limitada por los dos extremos deterministas. En este caso, podría ser posible tomar la decisión final en base a una aproximación a riesgo neutro que use valores medios para obtener el coste mínimo representado por el punto (3) en dicha figura. 
Sin embargo, como la incertidumbre se obtiene en base a intervalos de tolerancia/confianza, también es posible tomar la decisión final considerando una aproximación adversa al riesgo seleccionando aquel punto con el límite superior de indisponibilidad del intervalo de tolerancia/confianza igual al máximo valor de indisponibilidad asumible, por ejemplo el punto (4) en la Figura 7-16. Para este último caso, el valor del coste asociado es mayor que el coste correspondiente a la opción de riesgo neutro. Se ve aquí la importancia de tratar la incertidumbre de la efectividad del mantenimiento en la optimización de pruebas y mantenimientos, en particular para tomar decisiones con un punto de vista de adverso al riesgo.

\subsubsection{Resultados de la optimización considerando la influencia del modelo PAS/PAR.}

La adopción del modelo más apropiado PAS o PAR, para las bombas y válvulas también introduce incertidumbre que afecta al resultado final del problema de optimización. Para tener en cuenta este tipo de incertidumbre en este caso de aplicación, la selección de modelo de mantenimiento imperfecto se caracteriza por una distribución dicotómica del parámetro $p$, donde $p$ y (1p) son las probabilidades de adoptar un modelo de mantenimiento imperfecto PAS o PAR, respectivamente, según la expresión (3-31), en el aparatado 3.8.1.

La Figura 7-17 muestra cómo se obtienen diferentes fronteras óptimas de Pareto cuando se adoptan diferentes valores de $p$. En este caso se mantiene constantes $\varepsilon_{p}$ y $\varepsilon_{v}$ con un valor igual a 0,5 .

La Figura 7-17 muestra como para valores bajos de costes la consideración de un modelo de mantenimiento PAS o PAR, $p=0$ ó $p=1$, para todos los componentes del sistema resulta en una gran diferencia para la indisponibilidad asociada, ver puntos (1) y (2). El resultado de la optimización obtenido usando valores intermedios para la probabilidad asignada al modelo PAS permanece dentro de las dos situaciones extremas. La diferencia entre las fronteras de Pareto alcanzadas cambiando la probabilidad muestra la importancia de escoger un modelo de mantenimiento imperfecto adecuado en la formulación del problema de optimización. 


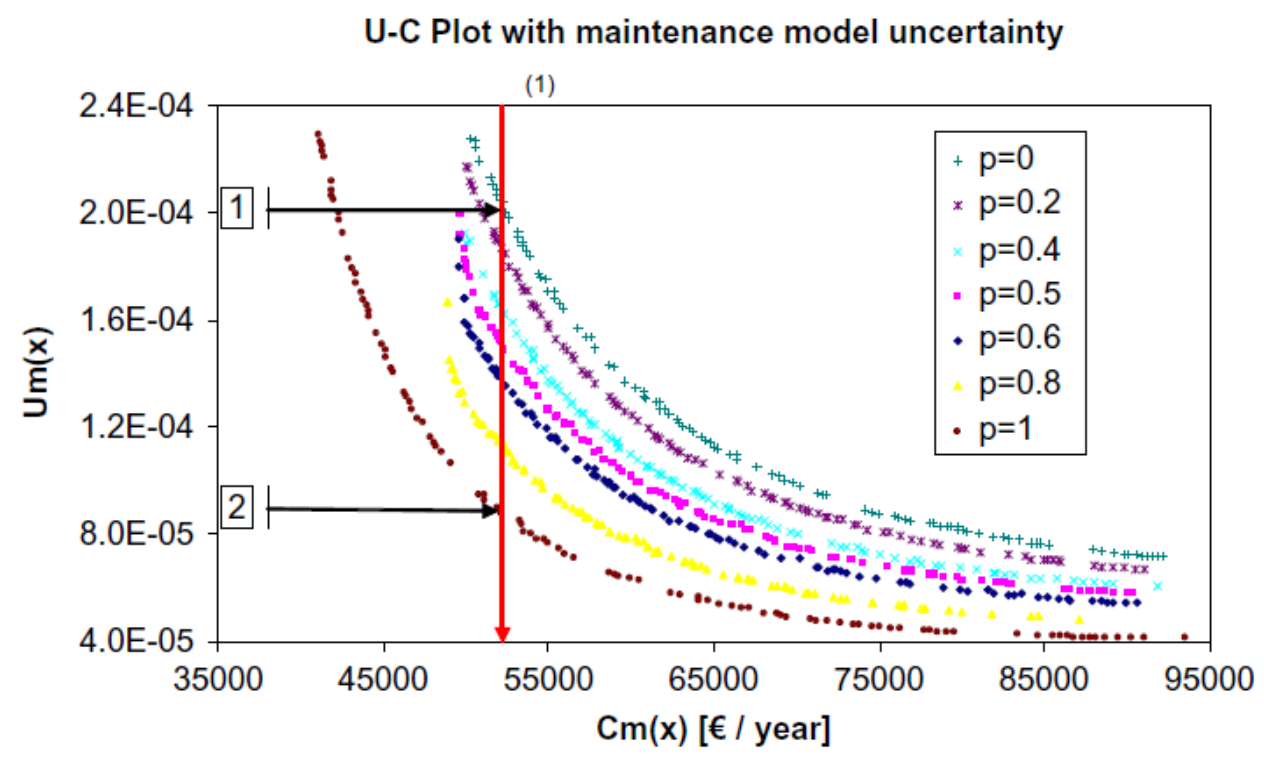

Figura 7-17: Análisis de sensibilidad para la selección del modelo de mantenimiento imperfecto.

\subsubsection{Resultados de la optimización considerando incertidumbres en el parámetro \& y modelo PAS/PAR.}

Finalmente se considera la incertidumbre tanto en el parámetro $\varepsilon$ como en el modelo de mantenimiento PAS/PAR. En este caso se selecciona una probabilidad $p=0,5$ constante ya que no hay evidencia de que ninguno de los modelos PAS o PAR sea más probable que el otro para simular el mantenimiento imperfecto de cualquier componente. Para los parámetros de efectividad de dichos mantenimientos, $\varepsilon_{p}$ y $\varepsilon_{v}$, se asignan una distribución aleatoria uniforme en ellos rangos $[0,5,0,9]$ y $[0,5,0,8]$ respectivamente.

La Figura 7-18 muestra el resultado de la optimización considerando $\varepsilon$ y la incertidumbre en el modelo de mantenimiento imperfecto comparado con los resultados obtenidos considerando sólo incertidumbre en el parámetro $\varepsilon$, Figura 7-16, obtenido en la sección 7.3.3.1.

La Figura 7-18 muestra que la consideración de las incertidumbres en el modelo de mantenimiento imperfecto y en la efectividad del mantenimiento simultáneamente proporciona una Frontera de Pareto con valores menores 
para ambos criterios, indisponibilidad y coste que si se considera únicamente incierta la efectividad del mantenimiento. Desde el punto de vista adverso al riesgo, se puede observar que para el máximo nivel de indisponibilidad aceptable, por ejemplo $1,5 \cdot 10^{-4}$, la consideración del modelo de mantenimiento imperfecto PAS/PAR y la efectividad del mismo simultáneamente proporciona un límite de tolerancia mayor con un coste menor asociado en comparación con el obtenido considerando solo incertidumbre en la efectividad del mantenimiento.

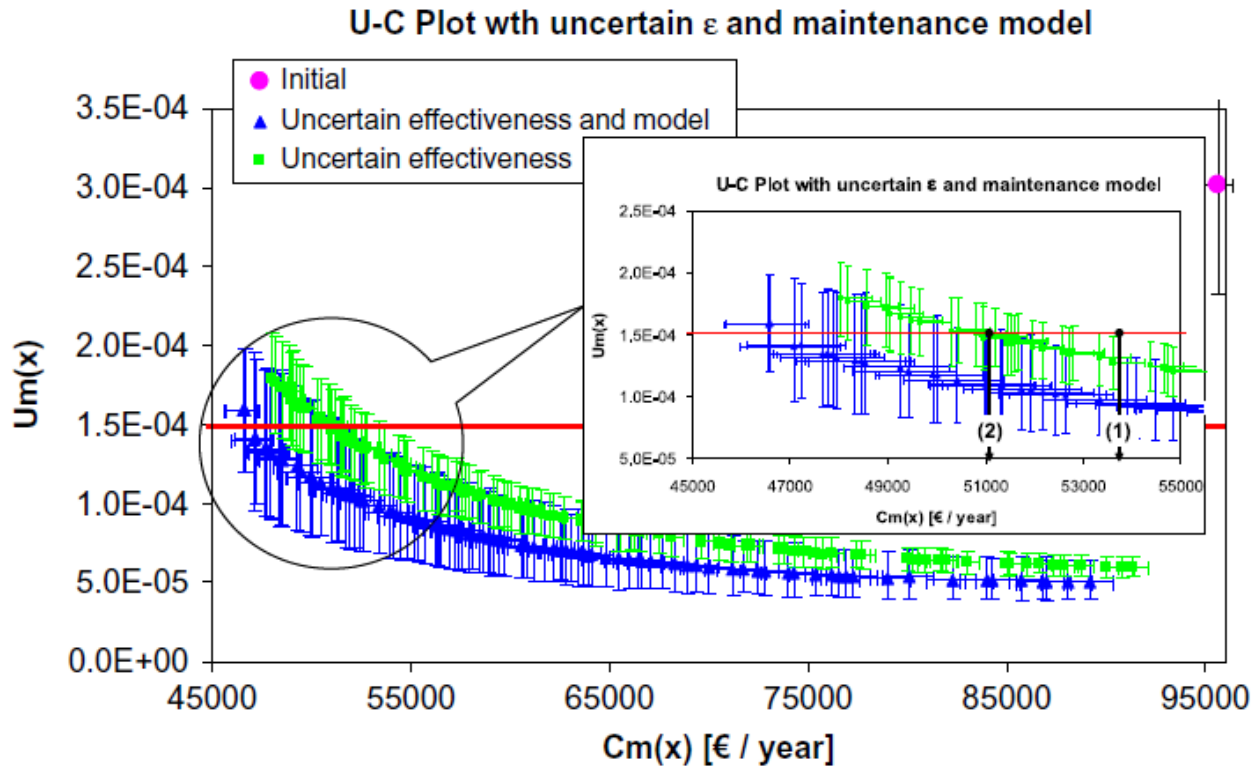

Figura 7-18: Comparación de los resultados de optimización considerando $\varepsilon$ y $\varepsilon$ + incertidumbre en el modelo de mantenimiento imperfecto.

De nuevo se puede seleccionar una decisión final basada en una aproximación a riesgo neutra, ver punto (2) en la Figura 7-18. Por otro lado también se puede seleccionar una decisión final basada en la aproximación adversa al riesgo representada por el punto (1) de la Figura 7-18 ya que la metodología explicada proporciona ambos resultados. 


\subsubsection{Discusión}

Los modelos usados en la cuantificación de los criterios adoptados en la optimización de las pruebas y mantenimientos presentan una gran dependencia de los valores seleccionados de parámetros, referentes a la fiabilidad de los componentes, efectividad del mantenimiento, etc. Tradicionalmente, el valor de estos parámetros se estima en base a un juicio de expertos y/ o por estimación mediante datos reales y asumiendo valor constate. Sin embargo esta hipótesis no es realista debido a que la incertidumbre asociada a los parámetros del modelo modifica la cuantificación de los criterios.

En esta aplicación, se ha llevado a cabo la optimización de las pruebas y mantenimientos basados en los criterios de indisponibilidad y costes y asumiendo incertidumbres en el modelo de efectividad del mantenimiento. En el proceso de optimización, se ha usado una aproximación mediante intervalos de tolerancia para cuantificar la incertidumbre, resolviendo el problema mediante una AGMO.

Los resultados obtenidos han mostrado la importancia de considera las incertidumbres en el parámetro $\varepsilon$ y en el modelo PAS/PAR, ya que las soluciones encontradas son considerablemente diferentes y de gran influencia en la toma de decisiones final. 


\section{Conclusiones y trabajo futuro}

\subsection{Conclusiones}

En la presente tesis se han propuesto varias aproximaciones para modelar el comportamiento de los equipos en base a los atributos RAMS+C; en primer lugar utilizando aproximaciones mediante valores promediados que son los más comunes dentro de la metodología de análisis probabilista de riesgos implantada en las centrales nucleares de todo el mundo. Dichos modelos se han usado para la optimización de las pruebas y el mantenimiento de equipos y sistemas en base a criterios RAMS $+C$. El problema de optimización planteado es un problema multiobjetivo sujeto a restricciones que afectan tanto en las variables de decisión como a los criterios adoptados. La resolución de dicho problema se ha llevado a cabo mediante el uso de Algoritmos Genéticos Múltiple Objetivo (AGMO), en concreto se ha utilizado el algoritmo SPEA2 adaptado a las necesidades del nuevo problema planteado, aplicado al caso concreto de los generadores diesel de emergencia de una central nuclear

En la siguiente etapa, se ha avanzado en la dirección de proponer nuevos modelos que han tenido en cuenta la variable tiempo en el comportamiento de los equipos desde el punto de vista RAMS+C. En este caso se ha propuesto una modelización más exacta del efecto de las diferentes 
estrategias de pruebas y mantenimiento de los equipos integrados a nivel de sistema, que permiten controlar los efectos que dichas estrategias tienen sobre la evolución temporal de los atributos RAMS+C. De esta manera se ha pretendido cuantificar las fuertes variaciones que ocurren de manera singular en determinados instantes de tiempo y, al contrario, que basándose en dicha información, el algoritmo de optimización sea capaz de buscar las mejores estrategias. Ello ha requerido adaptar los AGSO y AGMO para manejar nuevos criterios y variables de decisión, y además integrar mejoras en los algoritmos de optimización que los haga más eficientes. En concreto en esta tesis se ha utilizado como AGMO, el SPEA2, para la optimación la vigilancia bajo criterios RAMS $+C$ para el caso particular del sistema de inyección de agua a presión de una central nuclear.

Por último, en la tercera etapa, se han propuesto nuevos modelos y métodos que consideran el efecto de las incertidumbres asociadas a los parámetros del modelo, al propio modelo, e incluso a las variables de decisión en el proceso de optimización. Para ello se ha estudiado la formulación de las incertidumbres dentro de los modelos RAMS+C, cómo éstas influyen en la cuantificación de los atributos RAMS+C y cómo llevar a cabo la optimización de las pruebas y el mantenimiento en presencia de incertidumbres.

En primer lugar, se ha utilizado la aproximación probabilista clásica para formular las incertidumbres. En segundo lugar, se ha utilizado Monte Carlo como método de propagación de incertidumbres. Sin embargo, para reducir el esfuerzo computacional, se ha combinado el uso del método de Montecarlo con muestreo puro con el uso de estadísticos de orden. Esta combinación ha logrado obtener límites e intervalos de tolerancia/confianza para la estimación de los atributos RAMS $+C$, que permite tener una precisión suficiente y al mismo tiempo reducir apreciablemente el número de muestras necesarias a generar mediante Montecarlo. Y por último se han adaptado los algoritmos genéticos utilizados en trabajos previos para manejar criterios y variables de decisión inciertos, que son estimados mediante los anteriores límites e intervalos de tolerancia/confianza. Se ha analizado el efecto de la incertidumbre en las variables de decisión, en los parámetros de los modelos y en el modelo de mantenimiento imperfecto desarrollado. En los tres casos la aplicación se ha centrado en la optimización de los requisitos de vigilancia y mantenimiento para el sistema de inyección de agua a alta presión de una central nuclear típica.

En todos los casos se han aportado casos de aplicación de los nuevos modelos y métodos que demuestran la viabilidad y aplicabilidad de los mismos. 


\subsection{Aportaciones}

La presente tesis ha generado las siguientes aportaciones a nivel de artículos, capítulos de libro, congresos internacionales y congresos nacionales.

\subsubsection{Artículos}

1.1. Autores (p.o. de firma): Martorell S., Villanueva J.F., Carlos S., Nebot Y., Sanchez A., Pitarch J.L., Serradell V.

Título: RAMS+C informed decision-making with application to multiobjective optimization of technical specifications and maintenance using genetic algorithms

Ref: Reliability Engineering \& System Safety

Volumen 87 Páginas, inicial: 65 final: 75

Fecha: 2005.

(http://www.sciencedirect.com/science/article/B6V4T-4CKFK182/2/ea1452c76c003a2ce7d551415e8759d5 )

1.2. Autores (p.o. de firma): Martorell S., Carlos S., Villanueva J.F., Sanchez A., Galvan B., Salazar D.,Cepin M.

Título: Use of multiple objective evolutionary algorithms in optimizing surveillance requirements

Ref.: Reliability Engineering \& System Safety

Volumen 91 Páginas, inicial: 1027 final: 1038 Fecha: 2006.

(http://www.sciencedirect.com/science/article/B6V4T-4J021KT-

2/2/c4b9b0a46d0d696a01ffeda21a1d48ce)

1.3. Autores (p.o. de firma): Martorell S.; Sanchez A.; Villanueva J.F.; Carlos S.; Serradell V.

Título: A multi-objective genetic algorithm for RAMS $+C$ optimization with uncertain decision variables

Ref.: Journal of Risk and Reliability - 1748-006X

Volumen: 222 Páginas, inicial: 153 final: $160 \quad$ Fecha: 2008

(http://journals.pepublishing.com/content/n84t746146357351/?p=b64

d47a5ea68494397137846008d1c03\&pi=5) 
1.4. Autores (p.o. de firma): Villanueva JF; Sánchez A.; Carlos S.; Martorell S.

Título: Genetic algorithm-based optimization of testing and maintenance under uncertain unavailability and cost estimation: $A$ survey of strategies for harmonizing evolution and accuracy

Ref.: Reliability Engineering \& System Safety - 0951-8320

Volumen: 93 Páginas, inicial: 1830 final: $1841 \quad$ Fecha: 2008

(http://www.sciencedirect.com/science/article/B6V4T-4S3S2DT1/2/e4da4884e479c280ebaaf134cd5072ed)

1.5. Autores (p.o. de firma): Sanchez A.; Carlos S.; Martorell S.; Villanueva JF.

Título: Addressing imperfect maintenance modeling uncertainty in unavailability and cost based optimization

Ref.: Reliability Engineering \& System Safety - 0951-8320

Volumen: 94 Páginas, inicial: 22 final: $32 \quad$ Fecha: 2009

(http://www.sciencedirect.com/science/article/B6V4T-4NB99CV1/2/c558b4949f746f00be0fd7598d8bdb9d )

\subsubsection{Capítulo libro}

2.1. Título: Genetic Algorithms applications in Surveillance and Maintenance Optimization

Autores: S. Martorell; S. Carlos; A. I. Sanchez; J.F. Villanueva

Ref: Computational Intelligence in Reliability Engineering -978-354037367-4

Capítulo: 3 Páginas, inicial: 63 final: 99Fecha: 2007

Editorial: Springer

2.2. Título: Maintenance modeling and its optimization applied to safetyrelated equipment at nuclear power plants.

Autores (p.o. de firma): S. Martorell; JF Villanueva; S. Carlos; A. Sanchez

Ref: Safety and Reliability of Industrial Products, Systems and Structures -978-0415-66392-2

Capítulo: 23 Páginas, inicial: 277 final: $294 \quad$ Fecha: 2010

Editorial: CRC/Balkema Taylor and Francis Group 


\subsubsection{Congresos Internacionales}

3.1. Congreso: European Safety and Reliability Conference (ESREL) 2004 Annual Conference - PSAM 7

Título: Current Trends in Risk-Informed changes to Limiting Conditions for Operation

Fecha:2004 Lugar: Berlín, Alemania

Tipo de participación: Artículo

Autores: S. Martorell; J.F. Villanueva; Y. Nebot; S. Carlos; V. Serradell

3.2. Congreso: European Safety and Reliability Conference (ESREL) 2004 Annual Conference - PSAM 7

Título: From Risk to RAMS informed decision-making on changes to TSM

Fecha:2004 Lugar: Berlín, Alemania

Tipo de participación: Artículo

Autores: S. Martorell; J.F. Villanueva; Y. Nebot; S. Carlos; V. Serradell; A.I. Sánchez

3.3. Congreso: European Safety and Reliability Conference (ESREL) 2005 Annual Conference.

Título: Optimization of surveillance requirements at NPP considering time-dependent scheduling of tests and preventive

Fecha:2005 Lugar: Gdynia-Sopot-Gdansk, Poland

Tipo de participación: Artículo

Autores: S. Martorell; S. Carlos; J.F. Villanueva; A.I. Sánchez; C. Marko

3.4. Congreso: European Safety and Reliability Conference (ESREL) 2006 Annual Conference.

Título: A comparison of the use of single and multiple objectives in presence of uncertainty for testing and maintenance

Fecha:2006 Lugar: Estoril, Portugal

Tipo de participación: Artículo

Autores: S. Martorell; A.I. Sánchez; S. Carlos; J.F. Villanueva

3.5. Congreso: European Safety and Reliability Conference (ESREL) 2009 Annual Conference.

Título: Addressing uncertainties in risk-informed decision-making of changes to NPP technical specifications

Fecha:2009 Lugar: Praga, República Checa

Tipo de participación: Artículo

Autores: S. Martorell; A.I. Sánchez; M. Villamizar; J.F. Villanueva;

V. Serradell 
3.6. Congreso: II Jornadas Iberoamericanas de Asset Management Título: Tratamiento de las incertidumbres en la toma de decisiones de cambios en las ETFs informada en el riesgo en una planta nuclear

Fecha: $2009 \quad$ Lugar: Valencia, España

Tipo de participación: Artículo

Autores: S. Martorell; A.I. Sanchez; J.F. Villanueva; M. Villamizar; V. Serradell

3.7. Congreso: European Safety and Reliability Conference (ESREL) 2010. Reliability, Risk and Safety. Back to the future.

Título: Risk-informed decision-making on changes to allowed outage times addressing uncertainties

Fecha:2010 Lugar: Rodas, Grecia

Tipo de participación: Artículo

Autores: S. Martorell; M. Villamizar; JF Villanueva; S. Carlos; A. Sanchez

3.8. Congreso: The 7th International Conference on Engineering Computational Technology

Título: Particle Swarm Optimization: Application in Maintenance Optimization

Fecha: 2010

Lugar: Valencia, España

Tipo de participación: Artículo

Autores: S. Carlos; A. Sanchez; S. Martorell; JF Villanueva 


\subsubsection{Congresos Nacionales}

4.1. Congreso: VI Jornada de Fiabilidad. Gestión y Mejora Continua de la Confiabilidad en el Sector Industrial

Título: Optimización conjunta de frecuencias y estrategia de vigilancia de equipos de seguridad

Fecha:2004 Lugar: Madrid

Tipo de participación: Artículo

Autores: S. Martorell; A.I. Sánchez; S. Carlos; J.F. Villanueva

4.2. Congreso: XXVIII Congreso Nacional de Estadística e Investigación Operativa

Título: Integración de atributos de confiabilidad y coste en la toma de decisiones multicriterio

Fecha:2004

Lugar: Cádiz

Tipo de participación: Artículo

Autores: S. Martorell; J.F. Villanueva; S. Carlos; Y. Nebot; A.I. Sánchez; J.L. Pitarch

4.3. Congreso: VII Congreso de Confiabilidad. Jornada Técnica de Confiabilidad para la Calidad. Aplicaciones

Título: Optimización de intervalos de prueba considerando incertidumbre en la probabilidad de fallo del equipo

Fecha:2005

Lugar: Madrid

Tipo de participación: Artículo

Autores: S. Martorell; A.I. Sánchez; S. Carlos; J.F. Villanueva

4.4. Congreso: 31 Reunión de la Sociedad Nuclear Española.

Título: Optimización de intervalos de prueba en presencia de incertidumbres

Fecha:2005 Lugar: Logroño

Tipo de participación: Artículo

Autores: S. Martorell; S. Carlos; A.I. Sánchez; J.F. Villanueva; M. Cepin

4.5. Congreso: 31 Reunión de la Sociedad Nuclear Española.

Título: Optimización de requisitos de vigilancia considerando múltiples objetivos Fecha: $2005 \quad$ Lugar: Logroño

Tipo de participación: Artículo

Autores: S. Martorell; S. Carlos; A.I. Sánchez; J.F. Villanueva; M. Cepin 
4.6. Congreso: 32 Reunión de la Sociedad Nuclear Española.

Título: Optimización simple y múltiple objetivo de intervalos de prueba y mantenimiento en presencia de incertidumbres

Fecha:2006

Lugar: Tarragona

Tipo de participación: Artículo

Autores: S. Martorell; A.I. Sánchez; S. Carlos; J.F. Villanueva

4.7. Congreso: VIII Congreso de Confiabilidad. Jornada Técnica de Confiabilidad para la Calidad. Aplicaciones

Título: Estrategias de optimización basadas en criterios de confiabilidad y costes considerando incertidumbres. Lecciones aprendidas en la aplicación a la optimización de la vigilancia y el mantenimiento de equipos de seguridad

Fecha:2006 Lugar: Madrid

Tipo de participación: Artículo

Autores: S. Martorell; A.I. Sánchez; S. Carlos; J.F. Villanueva

4.8. Congreso: XXXIV Reunión Anual de la Sociedad Nuclear Española.

Título: Fuentes de incertidumbres en la cuantificación de medidas de riesgo utilizadas en el análisis de cambios de ETF.

Fecha:2008 Lugar: Murcia

Tipo de participación: Artículo

Autores: S. Martorell; A.I. Sánchez; S. Carlos; J.F. Villanueva; V. Serradell

4.9. Congreso: XXXV Reunión Anual de la Sociedad Nuclear Española.

Título: Análisis de cambios en STI del disparo del reactor informado en el riesgo teniendo en cuenta las incertidumbres.

Fecha:2009

Lugar: Sevilla

Tipo de participación: Artículo

Autores: S. Martorell; A.I. Sánchez; J.F. Villanueva; M. Villamizar;

S. Carlos; V. Serradell

4.10. Congreso: XXXV Reunión Anual de la Sociedad Nuclear Española.

Título: Análisis de cambios en AOT de acumuladores con información en el riesgo teniendo en cuenta las incertidumbres.

Fecha:2009 Lugar: Sevilla

Tipo de participación: Artículo

Autores: S. Martorell; A.I. Sánchez; J.F. Villanueva; M. Villamizar;

S. Carlos; V. Serradell 
4.11. Congreso: XXXVI Reunión Anual de la Sociedad Nuclear Española.

Título: Tratamiento de las incertidumbres en el análisis probabilista de cambios de STI.

Fecha:2010 Lugar: Santiago de Compostela

Tipo de participación: Artículo

Autores: S. Martorell; M. Villamizar; J.F. Villanueva; A.I. Sánchez;

S. Carlos; V. Serradell

4.12. Congreso: XXXVI Reunión Anual de la Sociedad Nuclear Española.

Título: Cuantificación de márgenes de seguridad para secuencias de APS.

Fecha:2010 Lugar: Santiago de Compostela

Tipo de participación: Artículo

Autores: S. Martorell; J.F. Villanueva; S. Carlos; M. Villamizar; A.I. Sánchez; V. Serradell

4.13. Congreso: XII Jornada de Confiabilidad para la Calidad.

Título: Análisis de cambios en AOT de Acumuladores con Información en el Riesgo teniendo en cuenta las incertidumbres.

Fecha: 2010 Lugar: Cádiz

Tipo de participación: Artículo

Autores: S. Martorell; M. Villamizar; A.I. Sánchez; J.F. Villanueva; S. Carlos 


\subsection{Trabajo futuro}

Esta tesis ha mostrado el interés de formular y resolver el problema de la optimización multiobjetivo de vigilancia y mantenimiento en centrales nucleares en base a criterios RAMS $+C$ como un todo. Sin embargo se necesita de un mayor estudio y nuevos casos de aplicación en este campo. La introducción de nuevas variables de optimización (redundancia en el diseño, elecciones de equipos equivalentes, etc.) puede ampliar el número de variables de decisión pero por otro lado las nuevas restricciones en los criterios de decisión pueden limitar el espacio de búsqueda (p.e. recursos humanos).

Una segunda línea para trabajos futuros concierne al desarrollo de la metodología y de herramientas que ayuden a la toma de decisiones. Es evidente que el tomador de decisiones debe tener como último paso un juicio de valor, sin embargo las dos alternativas típicas, POSO y POMO, que se han comparado en la presente tesis implementadas en un AGSO y AGMO respectivamente, representan opciones extremas en el proceso de toma de decisiones, esto es, tomar la decisión antes de la búsqueda para la alternativa POS (p.e. adoptando el AGSO) y tomar la decisión después de la búsqueda para la alternativa POM (p.e. adoptando AGMO). La primera puede conducir a tomar una decisión demasiado pronto y nuevas pruebas serían necesarias para guiar la búsqueda hacia otras regiones del espacio de búsqueda. Sin embargo la segunda puede conducir a tomar una decisión demasiado tarde, una vez que todo el espacio de búsqueda ha sido explorado, lo cual puede suponer un enorme esfuerzo computacional para finalmente observar que, por ejemplo, la frontera de Pareto no está tan bien definida y que solo una parte del espacio de búsqueda resulta de interés para el tomador de decisión. Una tercera vía basada en la toma de decisiones durante la búsqueda requiere de más estudios.

Existe un gran consenso sobre la bondad de los métodos basados en la llamada segunda generación de AGMO mostrando una alta eficiencia. Pero a pesar de los grandes avances que presentan estos algoritmos, los problemas de convergencia cuando se optimizan funciones objetivo computacionalmente costosas permanecen sin resolver. Este es el caso general de muchos de los problemas reales de optimización en la industria, y en particular, en el caso de la optimización de la vigilancia y mantenimiento basados en criterios RAMS $+C$ como los vistos en esta tesis. El uso de modelos dependientes del tiempo, métodos de simulación numérica o la incorporación de incertidumbres en la cuantificación de los atributos RAMS $+C$ aumenta drásticamente la complejidad y esfuerzo computacional necesario. Para tales problemas el coste computacional para evaluar cada solución candidata es muy costoso, 
normalmente de varios órdenes de magnitud (en tiempo de ejecución) mayores que el coste total de los lazos del AGMO. Es también bien conocido que los algoritmos evolutivos multiobjetivo pueden fallar al converger a la verdadera frontera de Pareto cuando esto sucede, por lo que los tomadores de decisión son reluctantes a su utilización y prefieren confiar en la información proporcionada por otras metodologías, normalmente clásicas (Winter 2005).

Por tanto, se puede prever la necesidad de cambios importantes en el desarrollo y aplicación de algoritmos genéticos para la optimización de vigilancia y mantenimiento para problemas más complejos (p.e. incremento del número de variables de decisión, objetivo y restricción), proveyendo flexibilidad en la toma de decisiones y considerando criterios RAMS $+C$ inciertos. Además las aproximaciones basadas en algoritmos genéticos han de asegurar una convergencia fiable y rápida para llegar a una buena solución.

Algunas alternativas para mejorar la convergencia apuntan hacia la incorporación de métodos de optimización híbridos, como los presentados en (Neittaanmaki 2005). Esta aproximación combina dos algoritmos acoplados para trabajar de forma cooperativa. Topología típica de hibridar algoritmos consisten en parejas heurístico-heurístico (p.e. AG-AG, AG-Búsqueda multidireccional, etc.) o heurístico-tradicional (AG-método del intervalo, AGmétodo del gradiente, etc.).

La mejora en la ejecución del AG pasa por una selección apropiada de los operadores genéticos y ajuste de los parámetros, tanto para el AGSO como para el AGMO. Sin embargo, ésta es una tarea que normalmente se lleva acabo de forma manual. Como consecuencia del tamaño y complejidad de los problemas RAMS+C, el analista tiene más probabilidades de fallar en este proceso, por lo que parece necesario buscar métodos que eviten o minimicen estos errores de usuario en la ejecución de los AG. Esto podría requerir un cambio en profundidad en la estructura y operación de los algoritmos genéticos.

La influencia del método para manejar las restricciones sobre el correcto funcionamiento de los algoritmos con base genética son de sobra conocidos, ya que provienen del hecho de que los AA.GG. son esencialmente técnicas de búsqueda sin restricciones que requieren de una adaptación para manejar las restricciones impuestas por el RAMS $+C$. Se debería trabajar en proponer nuevos métodos para manejar las restricciones, con particular atención a proveer de flexibilidad en el manejo de los rangos del espacio de decisión. 
Dentro de la incorporación de las incertidumbres en la toma de decisiones, los futuros trabajos deben concentrarse en reducir aún más el esfuerzo computacional, asegurando al mismo tiempo la convergencia al conjunto real de Pareto en presencia del ruido introducido por la incertidumbre, en orden a proveer una herramienta robusta, rápida y poderosa para la optimización multiobjetivo de la vigilancia y el mantenimiento informada por RAMS $+C$ bajo incertidumbres. Es precisamente en este terreno de la optimización de requisitos de pruebas y mantenimientos en presencia de incertidumbres aleatorias y epistémicas donde existe un amplio potencial en la mejora de la seguridad de las centrales nucleares. Por un lado, porque la optimización se debería focalizar a nivel de seguridad, puesto que las metas y requisitos propuestos por el regulador se sitúan a nivel de planta. Por ello, por otro lado, el aumento del nivel, pasando de sistema a planta, incrementa significativamente la incertidumbre de parámetros, y sobre todo, la de modelo, y completitud, lo cual requiere un esfuerzo de investigación muy importante. 


\section{Referencias}

Apostolakis, G. E. (1993). A comentary on model uncertainty. En A. Moshle, N. Siu, C. Smidts, \& C. Liu (Ed.), Workshop on model uncertainty: its characterization and quantification. Annapolis, MD: Center for Reliability Engineering. University of Maryland. College Park.

Atefi, B., \& alt., e. (1991). Feasibility assesment of a risk-based approach to technical specifications. . NUREG/CR-5742 (Vol. 1 y 2. Science Applications International Corporation).

Bäck, T. (1996). Evolutionary Algorithms in Theory and Practice. Evolution Strategies, Evolutionary Programming, Genetic Algorithms. New York: Oxford University Press.

Barata, J., Soares, C., Marseguerra, M., \& Zio, E. (2002). Simulation modelling of repairable multi-component deteriorating systems for 'on condition' maintenance optimization. Reliability Engineering and Safety System, 76, 255-264.

Beasley, D., Bull, D., \& Martin, R. (1993). An overview of genetic algorithms: part 1, fundamentals. University Computing.

Beasley, D., Bull, D., \& Martin, R. (1995). An overview of genetic algorithms: part 2, research topics. University Computing. 
Borgonovo, E., Marseguerra, M., \& Zio, E. (2000). A Monte Carlo methodological approach to plant availbility modeling with maintenance, aging and obsolescence. Reliability Engineering and System Safety, 72, 59-74.

Bunea, C., \& Bedford, T. (2002). The effect of model uncertainty on maintenance optimiztion. IEEE Transactions on REliability, 51(4), 486-493.

Busacca, P. G., Marseguerra, M., \& Zio, E. (2001). Multiobjective optimization by genetic algorithms: application to safety systems. Reliability Engineering and System Safety, 74(1), 59-74.

Cantoni, W., Marseguerra, M., \& Zio , E. (2000). Genetic algorithms and Monte Carlos simulation for optimal plant design. Reliability Engineering and System Safety, 68(1), 29-38.

Carlson, S. (1995). A General Method for Handling Constraints in Genetic Algorithms. University of Virginia.

Carlson, S., Shonkwiler, R., Babar, S., \& Aral, M. (1995). Annealing a Genetic Algorithm over Constraints. University of Virginia.

Cepin, M. (2002). Optimization of safety equipment outages improves safety. Reliability Engineering and System Safety, 77, 71-80.

Cepin, M., \& Martorell, S. (2002). Evaluation of allowed outage time considering a set of plant configurations. Reliability Engineering and System Safety, 78(3), 259-266.

Charles, E., \& Kondo, A. (2003). Availability allocation to repariable systems with genetic algorithms: a multiple-objective formulation. Reliability Engineering and System Safety, 82(3), 319-330.

Coit, D. W., \& Smith, A. E. (1998). Redundancy allocation to maximize a lower percentile of the system time-to-failure distribution. IEEE Transactions on Reliability, 47(1), 79-87.

Coit, D. W., \& Smith, A. E. (2002). Genetic algorithm to maximize a lowerbound for system time-to-failure with uncertain component Weibull parameters. Computers and Industrial Engineering, 41(4), 423-440. 
Coit, D. W., Jin, T. D., \& Wattanapogsakorn, N. (2004). System optimization with component reliability estimation uncertainty: a multicriteria approach. IEEE Transactions on Reliability, 53(3), 369-380.

Davis, L. (1991). Handbook of Genetic Algorithms. New York: Van Nostrand Reinholt.

De Jong, K. (1975). An analysis of the behaviour of a class of genetic adaptive systems. PhD Thesis, University of Michigan.

Deb, K., Pratap, A., Agarwal, S., \& Meyarivan, T. (2002). A fast and elitist multiobjective genetic algorithm. NSAG-II. IEEE Transactions on Evolutionary Computation, 6(2), 182-197.

DIQN-UPV. (2001). GV-IN-001. Metodología de un sistema indicador de vida (SIV). Depto. Ingeniería Química y Nuclear - Universidad Politécnica de Valencia.

EPRI. (1989). EPRI NP 6152. Demonstration of reliability centered maintenance. First annual progress report from San Onofre Nuclear Generation Station. EPRI.

Fogel, D. (1994). An introduction to simulated evolutionary optimization. IEEE Transactions on Neural Networks, 15(1), 3-14.

Fogel, D. B. (1995). Evolutionary Computation. New York: IEEE Press.

Fonseca, C. M., \& Fleming, P. J. (1993). Genetic algorithms for multiobjective optimization: Formulation, discussion and feneralization. Fifth International Conference on Genetic Algorithms (págs. 416-423). San Mateo, California: Morgan Kaufmann.

Goldberg, D. E. (1989). Genetic Algorithms in search, optimization and machine learning. Reading, MA: Addison-Wesley Pub. Co.

Guba, A., Makai, M., \& Pal, L. (2003). Statistical aspects of best estimated method. Relaibility Engineering and System Safety, 80(3), 2717-232.

Harunuzzaman, M., \& Aldemir, T. (1996). Optimization of standby safety system maintenance schedules in nuclear power plants. Nuclear Technology, 113(3), 354-367. 
Helton, J. C., \& Oberkampf, W. L. (2004). Alternative representations of epistemic uncertainty. Reliability Engineering and System Safety, 85(1-3), 1-10.

Herrera, F., \& Verdegay, J. L. (1996). Genetic Algorithms and Soft Computing. Heidelberg: Physica-Verlag.

Holland, J. (1975). Adaptation in Natural and Artifical Systems. University of Michigan Press.

Horn, J., Nafpliotis, N., \& Goldberg, D. E. (1994). A nitched Pareto genetic algorithm for multiobjective optimization. First IEEE Conference on Evolutionary Computation, IEEE World Congress on Computational Computation (págs. 82-87). Piscataway, NJ: IEEE Press.

International Atomic Energy Agency. (1992). IAEA-TECDOC-338. Methodology for the management of ageing of NPP components important to safety. IAEA.

International Atomic Energy Agency. (1993). IAEA-TECDOC-729. Risk-based optimization of technical specifications for operation of nuclear power plants. IAEA.

Joines, J. A., \& Houck, C. R. (1994). On the use of non-stationary penalty functions to solve nonlinear constrined optimization problems with GAs. IEEE World Congress on Computational Intelligence (págs. 579-584). Orlando: IEEE Press.

Kim, I. S., \& Martorell, S. (1994). Risk analysis of surveillance requeriments including their adverse effects. Reliability Engineering and System Safety, 45(3), 225-234.

Kim, I. S., Martorell, S., Veseley, W. E., \& Samanta , P. K. (1992). NUREG/CR-5775Quantitative evaluation of surveillance test intervals including test-caused risks. Brookhaven National Laboratory.

Knowles, J. D., \& Corne, D. W. (1999). The Pareto archived evolution strategy: A new baseline algorithm for Pareto multiobjective optimization. Congress on Evolutionary Computation. 1, págs. 98105. Piscataway, NJ: IEEE Press. 
Kumar, A., Pathak, R., \& Grupta, Y. (1995). Genetic-algorithms-based reliability optimization for computer network expansion. IEEE Transactions on Reliability, 44(1), 63-68.

Kursawe, F. (1991). A variant of evolution strategies for vector optimization. 1st Workshop Parallel Problem Solving from Nature (págs. 193-197). Berlin: Springer.

Lakkso, K. (1990). Optimization of technical specifications by probabilistic methods - a nordic perspective. Espoo, Finland: Technical Research Center of Finland.

Lapa, C. F., Pereira, C. N., \& Melo, P. F. (2000). Maximization of a nuclear system availability through maintenance scheduling optimization, using a genetic algorithm. Nuclear Engineering and Design, 196, 219231.

Lapa, C. F., Pererira, C. N., \& Melo, P. F. (2003). Surveillance test policy optimization through genetic algorithms using non-periodic intervention frequencies and considering seasonal constraints. Reliability Engineering and System Safety, 81(1), 103-109.

Lapa, C. M., \& Pereira, C. N. (2006). A model for preventive maintenance planning by genetic algorithms based in cost and reliability. Reliability Engineering and System Safety, 91(2), 233-240.

Laumanns, M., Thiele, L., Deb, K., \& Zitzler, E. (2002). Archiving with guaranteed convergence and diversity in multi-objective optimization. Genetic and EVolutionary Computation Conference (págs. 439-447). New York: Morgan Kaufmann Publishers.

Leemis, L. (1995). Reliability. Probabilistics Models and Statistical Methods. Englewood Cliffs, NJ: Prentice-Hall.

Levitin, G., \& Lisnianski, A. (1999). Joint redundancy and maintenance optimization for multistage series-parallel systems. Reliability Engineering and System Safety, 64(1), 33-42.

Mankamo, T. (1993). A risk-based approach to AOTs (Vol. 93). Finland: Avaplan Oy. 
Marseguerra, M., Zio, E., Podofillini, L., \& Coit, D. W. (2005). Optimal design of reliable network system in presence of uncertainty. IEEE Transactions on Reliability, 54(2), 243-253.

Marseguerra, M., \& Zio, E. (2000). Optimizing Maintenance and repair policies via a combination of genetic algorithms and Monte Carlo simulation. Reliability Engineering and System Safety, 68(1), 69-83.

Marseguerra, M., Zio, E., \& Martorell, S. (2006). Basics of genetic algorithms optimization for RAMS applications. Reliability Engineering and System Safety, 91(1), 977-991.

Marseguerra, M., Zio, E., \& Podofillini, L. (2002). Condition-based maintenance optimization by means of genetic algorithms and Monte Carlo simulation. Reliability Engineering and System Safety, 77, 151166.

Marseguerra, M., Zio, E., \& Podofillini, L. (2004). A multiobjective genetic algorithm approach to optimiation of the technical specifications of a nuclear safety system. Reliability Engineering and System Safety, 84(1), 87-99.

Marseguerra, M., Zio, E., \& Podofillini, L. (2004). Optimal reliability/availability of uncertain system via multi-objetive genetic algorithms. IEEE Transactions on Reliability, 53(3), 424-434.

Martorell, S., Carlos, S., \& Sánchez, A. (2007). A tolerance interval based approach to address uncertainty for RAMS $+C$ optimization. Reliability Engineering And System Safety, 92(4), 408-422.

Martorell, S., Carlos, S., Sánchez, A. I., \& Serradell, V. (2000). Constrained Optimization of test intervals using a steady-state genetic algorithm. Reliability Engineering and System Safety, 67(3), 215-232.

Martorell, S., Carlos, S., Sánchez, A. I., \& Serradell, V. (2002). Simultaneous and multicriteria optimization of TS requerimets and maintenance at NPPs. Annals of Nuclear Energy, 2(29), 147-168.

Martorell, S., Carlos, S., Villanueva, J., Sánchez, A., Galván, B., Salazar, D., y otros. (2006). Use of multiple objective evolutionary algorithms in optimizing surveillance requirements. Reliability Engineering and System Safety, 91, 1027-1038. 
Martorell, S., Sánchez, A. I., \& Serradell, V. (1999). Age-dependent reliability model considering effects of maintenance and working conditions. Reliability Engineering and System Safety, 64(1), 19-31.

Martorell, S., Sánchez, A. I., Carlos, S., \& Serradell, V. (2004). Alternatives and challenges in optimizing industrial safety using genetic algorithms. Reliability Engineering and System Safety, 86(1), 25-38.

Martorell, S., Sánchez, A. I., Muñoz, A., Pitarch, J. L., Serradell, V., \& Roldan, J. (1999). The use of maintenance indicators to evaluate the effects of maintenance programs on NPP perfomance and safety. Reliability Engineering and System Safety, 65(2), 85-94.

Martorell, S., Serradell, V., \& Samanta, P. K. (1995). Improving allowed outage time and surveillance test interval requeriments-a study of their interactions using probabilistic methods. Reliability Engineering and System Safety, 47(2), 119-129.

Martorell, S., Serradell, V., \& Verdú, G. (1996). Safety-related equipment prioritization for reliability centered maintenance purposes based on a plant specific level 1 PSA. Reliability Engineering and System Safety, 52(1), 35-44.

Michalewicz, Z. (1995). A survey of constraint handling techniques in evolutionary computation methods. Fourth International Conference on Evolutionary Programming (págs. 135-155). San Diego, CA: Mc Donnell, JR. Reynolds, RG., Fogel, DB.

Michalewicz, Z. (1996). Genetic Algorithms + Data Structures = Evolution Programs. Berlin: Springer-Verlag.

Muñoz, A., Martorell, S., \& Serradell, V. (1997). Genetic Algorithms in optimizing surveillance and maintenance of components. Reliability Engineering and System Safety, 57(2), 107-120.

Muñoz, A., Martorell, S., \& Serradell, V. (1997). Numerical absolute and constrained optimization of maintenance based oj risk and cost criteria using genetic algorithms. ESREL'97, (págs. 1749-1756). Lisboa. 
Neittaanmaki, P. (2005). Hybrid optimization methods for industrial applications. Evolutionary and deterministic methods for design, optimization and control with applications to industrial and societal problems, EUROGEN 2005. Munich.

Nuclear Energy Institute. (2001). NEI 95-10. Industry guideline for implementing the requeriments of 10 CFR Part 54 the License Renewal Rule. Nuclear Energy Institute.

Paiton, L., \& Campbell, J. (1995). Genetics algorithms in optimization of systems reliability. IEEE Trasnctions on Reliability, 64(2), 172-178.

Pereira, C. N., \& Lapa, C. F. (2003). Parallel island genetic algorithm applied to a nuclear power plant auxiliary feedwater system surveillance test policy optimization. Annals of Nuclear Energy, 30, 1665-1675.

Reinert, J. M., \& Apostolakis, G. E. (2006). Including model uncertainty in riskinformed decision making. Annals of Nuclear Energy, 4(33), 354-369.

Rocco, C. M. (2002). Maintenance optimization under uncertainties using interval methods and evolutionary strategies. Annual Reliability and Maintainability Symposium, (págs. 254-259).

Rocco, C. M., Miller, A. J., Moreno, J. A., Carrasquero, N., \& Medina, M. (2000). Sensitivity and uncertainty analysis in optimization programs using an evolutionary approach: a maintenance application. Reliability Engineering and System Safety, 67(3), 249-256.

Samanta, P. K. (1995). NUREG/CAR-6141. Handbook of methods for riskbased analysis of technical specifications. Brookhaven National Laboratory.

Samanta, P. K., Wong, S. M., \& Carbonaro, J. C. (1988). NUREG/CR-5200. Evaluation of risk associated with AOT and STI requirements at the ANO-1 nuclear power plant. Brookhaven National Laboratoty.

Schaffer, J. D. (1985). Multiple objective optimization with vector evaluated genetic algorithms. International Conference on Genetic Algorithms and their Applications, (págs. 93-100). Pittsburgh, PA. 
Srinivas, N., \& Deb, K. (1994). Multiobjective optimization using nondominated-sorting in genetic algorithms. Evolutionary Computation, 2(3), 221-248.

Taylor, G. M. (1989). San Onofre: using RCM to optimize preventive maintenance. Nuclear News, 32(14), 49.

Tsai, Y. T., Wang, K. S., \& Teng, H. Y. (2001). Optimizing preventive maintenance for mechanical components using genetic algorithms. Reliability Engineering and System Safety, 74(1), 89-97.

UNESA. (1995). Sistema de evaluación de vida remanente de centrales nucleares. Programa de investigación y desarrollo tecnológico. PIE 044.037 (Fase I). UNESA.

US NRC. (1997). RG 1160. Monitoring the effectiveness of maintenance at nuclear power plants. US NRC.

US NRC. (1998). RG 1177. An approach for plant specific, risk-informed decision-making: technical specifications. US NRC.

US NRC. (2000). RG 1182. Assessing and managing risk before maintenance activities at nuclear power plants. US NRC.

US NRC. (2001). RG1188. Standard format and content for applications to renew nuclear power plant operating licenses. US NRC.

US NRC. (2002). RG 1174. An approach for using probabilistic risk assessment in risk-informed decissions on plant specific changues to the liceasing basis. US NRC.

Utyuzhnikov, S. V. (2005). Numrical method for generating the entiere Pareto frontier in multiobjective optimization. Evolutionary and deterministic methods for disgn, optimization and control with applications to industrial and societal problems, EUROGEN 2005. Munich.

Vaurio, J. K. (1995). Optimization of test and maintenance intervals based on risk and cost. Reliability Engineering and System Safety, 49(1), 2336. 
Vesely, W. (1999). Principles of resource-effectiveness and regulatoryeffectiveness for risk-informed applications: reducing burdens by improving effectiveness. Reliability Engineering and System Safety, 63(3), 283-292.

Vinod, G., \& Kushwaha, A. W. (2004). Optimization of ISI interval using genetic algorithms for risk informed in-service inspection. Reliability Engineering and System Safety, 86, 307-316.

Wilks, S. (1941). Determination of sample sizes for setting tolerance limits. Annals of Mathematical Statistics, 12(1), 91-96.

Winter, G., Galván, B., Alonso, A., \& Méndez, M. (2005). New trends in evolutionary optimization and its impact on depdability problems. Evolutionary and deterministic methods for design, optimization and conrol with applications to industrial and societal problems, EUROGEN 2005. Munich.

Winter, G., Galván, B., Alonso, S., \& González, B. (2002). Evolving from genetic algorithms to flexible evolution agents. En C. P. Erick (Ed.), Genetic and evolutionary computation conference, GECCO 2002 (págs. 466-473). San Mateo, California: Morgan Kaufmann Publishers.

Wu, S., \& Clements-Croome, D. (2005). Preventive maintenance models with random maintenance quality. Reliability Engineering and System Safety(90), 99-105.

Yang, J. E., Hwang, M. J., Sung, T. Y., \& Jin, Y. (1999). Application of genetic algorithm for reliability allocation in nuclear power plants. Reliability Engineering and System Safety, 65, 229-238.

Yang, J., Sung, T., \& Jin, Y. (2000). Optimization of the surveillance test interval of the safety systems at the pant level. Nuclear Technology, 132(3), 352-365.

Zio, E., \& Apostolakis, G. (1996). Two methods for the structured assesments pf model uncertainty by experts in perfomance assessments of radioactive waste repositories. Releability Engineering and System Safety(54), 225-241. 
Zitzler, E. (1999). Evolutionary Algorithms for Multiobjective Optimization: Methods and Applications. PHD Thesis, Swiss Federal Institute of Technology, Zurich.

Zitzler, E., Laumanns, M., \& Thiele, L. (2001). SPEA2: Improving the strength Pareto evolutionary algorithm. . TIK-report 2001, Computer Engineering and Networks Lab (TIK) Swiss Federal Institute of Technology, Zurich.

Zitzler, E., Laumanns, M., Thiele, L., Fonseca, C. M., \& Grunert da Fonseca, V. (2003). Perfomance assessment of multiobjective optimizers: an analisys and review. IEEE Transactions on Evolutionary Computation, 7(2), 117-132. 